Dissertation

\title{
Central sound encoding in the inferior colliculus of mouse models for human auditory synaptopathy and neuropathy
}

\author{
for the award of the degree \\ "Doctor rerum naturalium" of the \\ Georg-August-Universität Göttingen \\ within the doctoral program \\ "Sensory- and Motorneuroscience" \\ of the Georg-August University School of Science (GAUSS)
}

submitted by

Maike Pelgrim

born in Stuttgart

Göttingen, 2018 


\section{Members of the Thesis Committee}

PD Dr. Nicola Strenzke

Auditory Systems Physiology Group, Department of Otolaryngology, University Medical Center, Göttingen

Prof. Dr. Alexander Gail

Sensorimotor Group, German Primate Center, Göttingen

Dr. Manuela Schmidt

Somatosensory Signaling Group, Max-Planck-Institute for Experimental Medicine, Göttingen

\section{Further members of the Examination Board}

Prof. Dr. Martin Göpfert

Department of Cellular Neurobiology, Schwann-Schleiden Research Center, Göttingen

Prof. Dr. Andrea Antal

Department of Clinical Neurophysiology, University Medical Center, Göttingen

Prof. Dr. Tim Gollisch

Sensory Processing in the Retina Group, Department of Ophthalmology, University Medical Center, Göttingen

Date of Disputation: 4th of December 2018 
Nicht sehen trennt uns von den Dingen. Nicht hören von den Menschen.

(Immanuel Kant) 



\section{Abstract}

Auditory neuropathy and auditory synaptopathy are hearing disorders with normal outer hair cell function and cochlear amplification, but an impairment in the function of inner hair cells, their ribbon synapses, or in the spiral ganglion neurons and neurons of the auditory pathway, respectively. In order to better understand the disease mechanisms, we analyzed central sound encoding in the inferior colliculus, the main auditory nucleus of the midbrain. We performed electrophysiological in vivo single unit recordings from the central nucleus of the inferior colliculus in three strains of mutant mice under isoflurane anesthesia. The first mouse line carried the I515T point mutation in the Otof gene, coding for otoferlin, which is expressed in the inner hair cell ribbon synapses where it is essential for synaptic vesicle exocytosis. This human relevant mutation leads to a deficit in synaptic vesicle reformation at the inner hair cell ribbon synapse, resulting in abnormal adaptation and delayed recovery from adaptation in spiral ganglion neurons despite normal thresholds. A similar but much more severe phenotype is caused by the D1767G point mutation ("pachanga"). Finally, we used a mouse line carrying the quiverer-3J mutation in the Spnb (synonym Sptbn 4) gene, coding for $\beta \mathrm{IV}$-spectrin, which is expressed in the axon initial segments and nodes or Ranvier of all neurons where it helps clustering sodium channels. Human mutations in $\beta \mathrm{IV}$-spectrin cause a neurological syndrome where hearing loss is accompanied by motor, cognitive and sensory dysfunction.

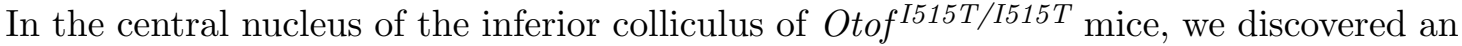
increased adaptation in response to amplitude modulated sounds with low modulation frequencies, as well as an increased depression of spiking after forward masking, which likely contributes to the speech perception deficit of patients with an I515T mutation in otoferlin. Furthermore, we could show that an increase in central gain, does not suffice to compensate the phenotype of mice with the Otof ${ }^{P g a / P g a}$ mutation. In addition to that, a delay of spiking caused by the $S p n b^{q v-3 J / q v-3 J}$ mutation leads to a use dependent decrease in the accuracy of spiking. This results in an increased variance of the first spike latency when stimulating with higher repetition rates and a reduced temporal precision of spiking in response to amplitude modulated tones, represented by a reduced maximal modulation frequency up to which phase-locking is possible in the inferior colliculus. Overall, our results help to explain why speech perception is poor in patients with auditory neuropathy and auditory synaptopathy despite well-preserved sound detection thresholds. A gap detection test should be well suited to detect the adaptation defects caused by otoferlin mutations, whereas in spectrin mutants, sound encoding deficits are expected to vary considerably with stimulation rate. We suggest that improvement in hearing function might not be achieved by designing hearing aids that amplify all sounds, but instead by increasing the speech to noise ratio and reducing sound levels to a minimum to prevent abnormal adaptation. 



\section{Zusammenfassung}

Auditorische Neuropathien und Synaptopathien sind Hörstörungen, die mit einer normalen Funktion der äußeren Haarzellen und einer normalen cochleären Verstärkung, aber mit eingeschränkter Funktion der inneren Haarzellen, ihrer Bändersynapsen oder der Spiralganglienneuronen und Neuronen der Hörbahn einhergehen. Um die Mechanismen besser zu verstehen, die zu diesen Erkrankungen führen, haben wir die zentrale Schallkodierung im Colliculus inferior, einem der wichtigsten auditorischen Kerngebiete des Mittelhirns, untersucht. Dafür haben wir bei drei genmanipulierten Mausstämmen elektrophysiologische in vivo Einzelfasermessungen im zentralen Kern des Colliculus inferior unter Isofluran-Anästhesie durchgeführt. Die erste Mauslinie trägt die I515TPunktmutation im Otof-Gen. Das daraus entstehende Protein Otoferlin kommt in den Bändersynapsen der inneren Haarzellen vor, wo es für die Exozytose synaptischer Vesikel notwendig ist. Diese, für den Menschen relevante Mutation führt zu einer verminderten Neuformierung der synaptischen Vesikel, was zu einer gestörten Adaptation und einer verzögerten Erholung an Spiralganglienneuronen trotz normaler Schwellenwerte führt. Ein ähnlicher, aber viel schwerwiegenderer Phänotyp wird durch die D1767GPunktmutation ("pachanga") verursacht. Darüber hinaus haben wir eine Mauslinie mit einer quiverer-3J Mutation des Spnb (Synonym Sptbn4) Gens untersucht. Das betroffene Protein, $\beta$ IV-Spektrin kommt hauptsächlich in den Axoninitialsegmenten und den Ranvierschen Schnürringen aller Neuronen vor und bündelt dort Natriumkanäle. Mutationen im humanen $\beta$ IV-Spektrin verursachen ein neurologisches Syndrom, bei dem ein Hörverlust von motorischen, kognitiven und sensorischen Dysfunktionen begleitet wird. Wie unsere Untersuchungen zeigen, verursacht die Otof ${ }^{I 515 T / I 515 T}$ Mutation eine erhöhte Adaptation auf amplitudenmodulierte Töne mit niedrigen Modulationsfrequenzen sowie eine erhöhte Depression der Aktionspotentialraten nach Vorwärtsmaskierung im Colliculus inferior, was wahrscheinlich bei den betroffenen Personen zu den Schwierigkeiten in der Sprachwahrnehmung beiträgt. Des Weiteren konnten wir zeigen, dass zentrale Verstärkungseffekte nicht ausreichen, um den Phänotyp von Mäusen mit der Otof Pga/Pga Mutation zu kompensieren. Die verminderte Weiterleitungsgeschwindigkeit, verursacht durch die Spnb ${ }^{q v-3 J / q v-3 J}$ Mutation, führte zu einer nutzungsbedingten Beeinträchtigung der zeitlichen Präzision der Aktionspotentiale, insbesondere bei höheren Wiederholraten zu Beginn eines Stimulus. Bei amplitudenmodulierten Tönen war die maximale Modulationsfrequenz, bis zu der eine Phasenkopplung im Colliculus inferior möglich ist, reduziert. Insgesamt tragen unsere Ergebnisse dazu bei, zu erklären warum die Sprachwahrnehmung bei Patienten mit auditorischen Neuropathien und Synaptopathien trotz gut erhaltener Hörschwellen schlecht ist. Um die durch Otoferlin-Mutationen verursachten Adaptationsprobleme zu erkennen, sollte ein Hörtest zur Erkennung von Pausen zwischen Tönen geeignet sein, während bei Spektrin-Mutanten vermutlich Defizite auftreten, bei der die Schallkodierung mit der Stimulationsrate stark variiert. Zusammenfassend kommen wir zu dem Schluss, dass eine Verbesserung der Hörfunktion nicht durch die Entwicklung von Hörgeräten erreicht werden kann, die alle Geräusche verstärken, sondern durch eine Verbesserung des Sprach-Rausch-Verhältnisses und die Reduzierung der Schallpegel auf ein Minimum, um eine abnormale Adaptation zu verhindern. 



\section{Contents}

Abstract $\quad$ V

$\begin{array}{ll}\text { List of Figures } & \text { XIII }\end{array}$

$\begin{array}{ll}\text { List of Tables } & \text { XV }\end{array}$

$\begin{array}{ll}\text { Abbreviations } & \text { XVII }\end{array}$

1. Introduction 1

1.1. The mammalian ear . . . . . . . . . . . . . . . 2

1.1.1. Outer and middle ear . . . . . . . . . . . . . . . 2

1.1.2. Inner ear and organ of Corti . . . . . . . . . . . . . 3

1.1.3. Inner hair cell ribbon synapse . . . . . . . . . . . . . . . 4

1.2. Spiral ganglion neurons (SGNs) . . . . . . . . . . . . . . 4

1.2.1. Sound encoding in spiral ganglion neurons . . . . . . . . . 5

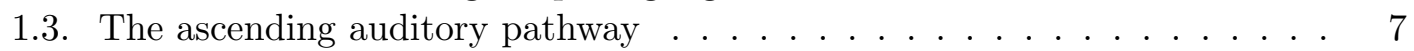

1.3.1. Anteroventral cochlear nucleus (AVCN) . . . . . . . . . 7

1.3.2. Superior olivary complex $(\mathrm{SOC}) \ldots \ldots$. . . . . . . . . 9

1.3.3. Ventral nucleus of the lateral lemniscus (VNLL) . . . . . . . . . 9

1.3.4. Inferior colliculus (IC) and its central nucleus (ICC) . . . . . . . 10

1.3.5. Medial geniculate body (MGB) . . . . . . . . . . . . . . 10

1.3.6. Auditory cortex $(\mathrm{AC}) \ldots \ldots \ldots 1$. . . . . . . . . . . 11

1.4. Sound encoding in the inferior colliculus . . . . . . . . . . . . . 11

1.4.1. Frequency selectivity . . . . . . . . . . . . . . . 11

1.4.2. Intensity coding . . . . . . . . . . . . . . . . 12

1.4.3. Response patterns . . . . . . . . . . . . . . . . 12

1.4.4. Phase-locking . . . . . . . . . . . . . . . . . . 12

1.4.5. Sound localization . . . . . . . . . . . . . . . 13

1.5. Single unit recordings from mice with auditory neuropathies and synap-

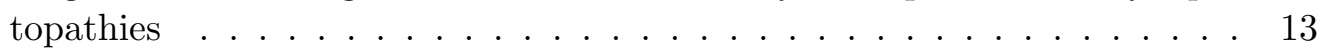

1.5.1. $B s n^{\Delta E x 4 / 5}$ mice . . . . . . . . . . . . . . . . . 14

1.5.2. Otof ${ }^{I 515 T / I 515 T}$ mice . . . . . . . . . . . . . . 15

1.5.3. Otof ${ }^{P g a / P g a}$ mice . . . . . . . . . . . . . . . . 17

1.5.4. Spnb ${ }^{q v-3 J / q v-3 J}$ mice $\ldots \ldots \ldots \ldots . \ldots \ldots$

2. Aim of the study 23 
3. Material and Methods 25

3.1. Animals . . . . . . . . . . . . . . . . . 25

3.1.1. Otof ${ }^{I 515 T / I 515 T}$ mice . . . . . . . . . . . . . 25

3.1.2. Otof ${ }^{P g a / P g a}$ mice . . . . . . . . . . . . . . . 26

3.1.3. Bsn ${ }^{f l / f l}$ Pres ${ }^{C r e}$ mice . . . . . . . . . . . . . . . . 26

3.1.4. Spnb ${ }^{q v-3 J / q v-3 J}$ mice . . . . . . . . . . . . . . . 26

3.2. Immunohistochemistry . . . . . . . . . . . . . . . . . . . 27

3.2.1. Preparation of the organ of Corti . . . . . . . . . . . 28

3.2.2. Fluorescent immunohistochemistry . . . . . . . . . . . . . 29

3.2.3. Preparation of brain cryoslices . . . . . . . . . . . . . . . 29

3.2.4. Confocal microscopy . . . . . . . . . . . . . . . . . . . . . 29

3.3. Auditory brainstem responses . . . . . . . . . . . . . . . . . 30

3.3.1. Recording procedure . . . . . . . . . . . . . . 30

3.4. In vivo single unit recordings from the inferior colliculus . . . . . . . . . . 31

3.4.1. Recording procedure . . . . . . . . . . . . . . 32

3.4.2. Stimulation protocol . . . . . . . . . . . . . 33

3.5. Data analysis . . . . . . . . . . . . . . . 36

3.5.1. Database and software design . . . . . . . . . . . . . 36

3.5.2. Frequency response analysis . . . . . . . . . . . . . . . 39

3.5.3. Level response analysis . . . . . . . . . . . . . . . . . . . . . . 39

3.5.4. Peri-stimulus-time histograms . . . . . . . . . . . . . . . 40

3.5.5. First spike latency . . . . . . . . . . . . . . . . . . . . 41

3.5.6. Amplitude modulation responses . . . . . . . . . . . . . . . . 41

3.5.7. Forward masking analysis . . . . . . . . . . . . . . . 42

3.6. Statistical analysis . . . . . . . . . . . . . . . . . . 42

3.7. Characterization of unit types in the inferior colliculus . . . . . . . . . . 43

3.7.1. Frequency response area . . . . . . . . . . . . . . . . . 43

3.7.2. Rate-level function . . . . . . . . . . . . . . . . . . . . . . . 44

3.7.3. Tone length . . . . . . . . . . . . . . . . . . . . . 44

3.7.4. Peri-stimulus time histogram . . . . . . . . . . . . . . 46

3.7.5. Amplitude modulation . . . . . . . . . . . . . . . . . . 46

3.7.6. Forward masking . . . . . . . . . . . . . . . . . 47

3.7.7. Frequency of unit types . . . . . . . . . . . . . 48

4. Results $\mathbf{5 1}$

4.1. Hair-cell specific bassoon knock-out mice $\left(B s n^{f l / f l}\right.$ Pres $\left.{ }^{C r e}\right) \ldots \ldots . . . .51$

4.1.1. Immunohistochemical stainings . . . . . . . . . . . . . . 51

4.1.2. Auditory brainstem responses . . . . . . . . . . . . . . . 52

4.1.3. Discussion and outlook . . . . . . . . . . . . . . . 53

4.2. Otoferlin Ile515Thr mice $\left(\right.$ Otof $\left.f^{1515 T / I 515 T}\right) \ldots \ldots \ldots$. . . . . . . 55

4.2.1. Tuning, thresholds and spontaneous rates are normal in ICNs of Otof ${ }^{I 515 T / I 515 T}$ mice . . . . . . . . . . . . . 55

4.2.2. Intensity coding is impaired in terms of a reduction in dynamic range 56

4.2.3. Coding of short tones is unaffected by the Otof ${ }^{I 515 T / I 515 T}$ mutation 58

4.2.4. Pure tone responses have a reduced and more jittered onset response 59

4.2.5. Forward masking is impaired in ICNs of Otof ${ }^{I 515 T / I 515 T}$ mice . . . 61 
4.2.6. Phase-locking to amplitude modulated tones is normal, but spike rates are strongly reduced, and adaptation is increased . . . . . . 61

4.3. Otoferlin pachanga mice $\left(O t o f^{P g a / P g a}\right) \ldots \ldots \ldots$. . . . . . . 67

4.4. Quiverer-3J mice $\left(S p n b^{q v-3 J / q v-3 J}\right) \ldots \ldots \ldots \ldots \ldots$

4.4.1. Spontaneous rate is reduced while tuning and thresholds are normal 70

4.4.2. Intensity coding is unaffected in $S p n b^{q v-3 J / q v-3 J}$ mice $\ldots \ldots . . .71$

4.4.3. Coding of short tones might be affected . . . . . . . . . . 73

4.4.4. Onset responses have a use dependent delay of spiking and increased jitter ...................... 73

4.4.5. Forward masking is unaffected in $S p n b^{q v-3 J / q v-3 J}$ mice $\ldots . . .777$

4.4.6. ICNs of $S p n b^{q v-3 J / q v-3 J}$ mice have a reduced maximal modulation frequency where phase-locking to sAM tones is possible . . . . . 77

5. Discussion $\mathbf{8 5}$

5.1. Part I: Methodological considerations . . . . . . . . . . . . . . . 85

5.2. Part II: Sound encoding in the ICC of mutant mice . . . . . . . . . . . 87

5.2.1. Frequency coding . . . . . . . . . . . . . . . . . 87

5.2.2. Spontaneous rate and intensity coding . . . . . . . . . . 88

5.2.3. Duration tuning . . . . . . . . . . . . . . . . . . . 89

5.2.4. Spike rates and spike timing . . . . . . . . . . . . 90

5.2.5. Compensatory mechanisms by increasing central gain . . . . . . . 91

5.2.6. Forward masking . . . . . . . . . . . . . . . 92

5.2.7. Precision of phase-locking in response to sAM tones . . . . . . . 93

5.2.8. Spike rates in response to sAM tones . . . . . . . . . . . . . . 94

5.3. Part III: Human auditory synaptopathies and neuropathies . . . . . . . 95

5.3.1. Human patients with non-syndromic auditory synaptopathy DFNB9 96

5.3.2. Human patients with syndromic auditory neuropathy . . . . . . . 97

5.3.3. Diagnosis and treatment . . . . . . . . . . . . . . . 99

5.4. Summary and Conclusion . . . . . . . . . . . . . . . 100

$\begin{array}{ll}\text { Appendix } & \text { XVIII }\end{array}$

$\begin{array}{ll}\text { A. References } & \text { XIX }\end{array}$

B. Statistics $\quad$ XXXV

$\begin{array}{lll}\text { C. Acknowledgment } & \text { XLIII }\end{array}$

$\begin{array}{ll}\text { D. Declaration } & \text { XLV }\end{array}$

$\begin{array}{ll}\text { E. Curriculum Vitae } & \text { XLVII }\end{array}$ 



\section{List of Figures}

1.1. Schematic view of the ear, cochlea and organ of Corti . . . . . . . . 2

1.2. Auditory pathway . . . . . . . . . . . . . . . 8

1.3. Auditory brainstem responses and SGN recordings from $B s n^{\Delta E x} 4 / 5$ mice 14

1.4. Auditory brainstem responses of Otof ${ }^{I 515 T / I 515 T}$ mice . . . . . . . . . 16

1.5. Responses from SGNs of Otof ${ }^{I 515 T / I 515 T}$ mice . . . . . . . . . . . . . . 17

1.6. Auditory brainstem responses of Otof ${ }^{P g a / P g a}$ mice . . . . . . . . . . . 18

1.7. Responses from SGNs of Otof ${ }^{P g a / P g a}$ mice . . . . . . . . . . . . . . 19

1.8. Auditory brainstem responses of $S p n b^{q v-3 J / q v-3 J}$ mice . . . . . . . . . 20

1.9. Responses from SGNs of $S p n b^{q v-3 J / q v-3 J}$ mice . . . . . . . . . . . 21

1.10. Responses of neurons from the AVCN of $S p n b^{q v-3 J / q v-3 J}$ mice $\ldots \ldots . .22$

3.1. Recording Procedure . . . . . . . . . . . . . . . . 33

3.2. Scheme of the Database . . . . . . . . . . . . . . . . 38

3.3. Dynamic Range . . . . . . . . . . . . . . . . . . . . . . . . . . . 40

3.4. Classes of FRAs . . . . . . . . . . . . . . . . . 44

3.5. Classes of rate-level functions . . . . . . . . . . . . . . . . . 45

3.6. Classes of tone length responses . . . . . . . . . . . . . . . . . . 45

3.7. Classes of PSTH shapes . . . . . . . . . . . . . . . . . 47

3.8. Classes of AM tone responses . . . . . . . . . . . . . . . . . 48

3.9. Classes of forward masking responses . . . . . . . . . . . . . . . . . 49

3.10. Frequency of different classes for each run type . . . . . . . . . . . 49

4.1. Staining of Ribeye and Bassoon on Bsn ${ }^{f / / f l}$ Pres ${ }^{C r e}$ mice . . . . . . . . . . 52

4.2. Staining of Ribeye and PSD-95 on Bsn ${ }^{f / f l}$ Pres ${ }^{C r e}$ mice . . . . . . . . . 53

4.3. Auditory brainstem responses of $B s n^{f l / f l}$ Pres ${ }^{C r e}$ mice . . . . . . . . . . 54

4.4. Spontaneous rate, tuning and thresholds are normal in Otof ${ }^{1515 T / I 515 T}$ mice 56

4.5. Rate-level functions from ICNs of Otof ${ }^{I 515 T / I 515 T}$ mice have a smaller dynamic range . . . . . . . . . . . . . . . . . 5 57

4.6. Responses to pure tones with different tone length are normal in ICNs from Otof ${ }^{I 515 T / I 515 T}$ mice . . . . . . . . . . . . . . . . 58

4.7. Onset responses are reduced and more jittered in ICNs of Otof $f^{1515 T / I 515 T}$

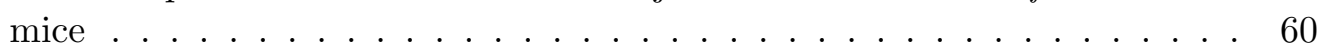

4.8. Depression of spiking in a forward masking paradigm is impaired in ICNs of Otof ${ }^{I 515 T / I 515 T}$ mice . . . . . . . . . . . . . . . . . . 61

4.9. Circle diagrams to depict phase-locking to amplitude modulated tones in ICNs from Otof I515T/I515T mice . . . . . . . . . . . . . . . 62

4.10. Responses to amplitude modulated tones with varying modulation frequencies from ICNs of Otof ${ }^{I 515 T / I 515 T}$ mice. . . . . . . . . . . . . . 63

4.11. Responses to amplitude modulated tones with varying sound intensities from ICNs of Otof $1515 T / I 515 T$ mice . . . . . . . . . . . . . . . . 
4.12. Responses to amplitude modulated tones with varying modulation depths from ICNs of Otof ${ }^{I 515 T / I 515 T}$ mice . . . . . . . . . . . . . . 66

4.13. Responses of a representative ICN from an Otof ${ }^{P g a / P g a}$ mouse . . . . . . 68

4.14. Frequency tuning, rate-level functions and PSTHs from ICNs of Otof Pga/Pga

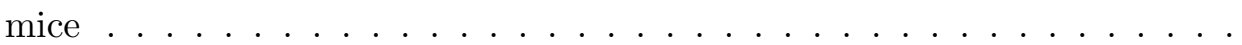

4.15. Spontaneous rate is reduced while tuning and thresholds are normal in ICNs of $S p n b^{q v-3 J / q v-3 J}$ mice . . . . . . . . . . . . . . . 71

4.16. Intensity coding is normal in $S p n b^{q v-3 J / q v-3 J}$ mice . . . . . . . . . . . 72

4.17. Coding of short tones might be affected in ICNs of $S p n b^{q v-3 J / q v-3 J}$ mice .73

4.18. Onset responses are reduced and delayed in ICNs of $S p n b^{q v-3 J / q v-3 J}$ mice .75

4.19. Use dependent increase in delay of spiking and in variance of first-spike latency in ICNs of $S p n b^{q v-3 J / q v-3 J}$ mice . . . . . . . . . . . . 76

4.20. Depression of spiking in a forward masking paradigm is unaffected in ICNs of $S p n b^{q v-3 J / q v-3 J}$ mice . . . . . . . . . . . . . . . 77

4.21. Circle diagrams to depict phase-locking to amplitude modulated tones in ICNs from $S p n b^{q v-3 J / q v-3 J}$ mice . . . . . . . . . . . . . 78

4.22. Responses to amplitude modulated tones with varying modulation frequencies from ICNs of Spnb qv-3J/qv-3J mice . . . . . . . . . . 79

4.23. Responses to amplitude modulated tones with varying sound intensities from ICNs of $S p n b^{q v-3 J / q v-3 J}$ mice . . . . . . . . . . . . . . . 81

4.24. Responses to amplitude modulated tones with varying modulation depths from ICNs of $S p n b^{q v-3 J / q v-3 J}$ mice . . . . . . . . . . . . . . . . . 


\section{List of Tables}

1. Animal numbers . . . . . . . . . . . . . . . . . . 25

3. Immunohistochemistry setup equipment . . . . . . . . . . . . . . . . 27

5. Immunohistochemistry material . . . . . . . . . . . . . . . . . . . . 27

7. Immunohistochemistry recipes . . . . . . . . . . . . . . . . . 28

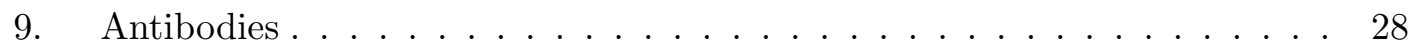

11. ABR Setup equipment . . . . . . . . . . . . . . . . 30

13. IC recording setup equipment . . . . . . . . . . . . . . . . . 31

15. IC recording consumables . . . . . . . . . . . . . . . . . . 32

17. Software . . . . . . . . . . . . . . . . . . 36

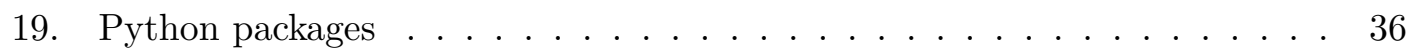

21. Software scripts provided by Marcus Jeschke . . . . . . . . . . . . . . . 37

23. Statistics of Otof ${ }^{I 515 T / I 515 T}$ mice . . . . . . . . . . . . . . XXXV

24. Statistics of $S p n b^{q v-3 J / q v-3 J}$ mice . . . . . . . . . . XXXXIII 



\section{Abbreviations}

\begin{tabular}{|c|c|}
\hline ABR & Auditory brainstem response \\
\hline $\mathrm{AC}$ & Auditory cortex \\
\hline AIS & Axon initial segment \\
\hline $\mathrm{AM} \ldots$ & Amplitude modulation \\
\hline AMPA & $\alpha$-amino-3-hydroxy-5-methyl-4-isoxazolepropionic acid \\
\hline $\mathrm{AN} \ldots$ & Auditory nerve \\
\hline $\mathrm{AVCN} \ldots$ & Anteroventral cochlear nucleus \\
\hline $\operatorname{Bsn}^{\Delta \operatorname{Ex} 4 / 5 \ldots} \ldots$ & $\Delta \mathrm{Ex} 4 / 5$ bassoon mutation \\
\hline Bsn ${ }^{\mathrm{fl} / \mathrm{fl}}$ Pres ${ }^{\mathrm{Cre}}$ & Hair-cell specific bassoon knock out \\
\hline CAP ......... & Compound action potential \\
\hline $\mathrm{CF}$. & Characteristic frequency \\
\hline dBOpt . & Intensity at $\mathrm{CF}$ which elicits the maximal spike rate \\
\hline DPOAE & Distortion product of otoacoustic emission \\
\hline FA . & Formaldehyde \\
\hline FRA & Frequency response area \\
\hline FSL $\ldots$ & First spike latency \\
\hline GABA . & $\gamma$-aminobutyric acid \\
\hline GSDB & Goat serum dilution buffer \\
\hline GUI ... & Graphical user interface \\
\hline IC . & Inferior colliculus \\
\hline ICC & Central nucleus of the inferior colliculus \\
\hline $\mathrm{ICN}$ & Inferior colliculus neuron \\
\hline IHC .... & Inner hair cell \\
\hline ILD ..... & Interaural level difference \\
\hline ITD .. & Interaural time difference \\
\hline LNTB . . & Lateral nucleus of the trapezoid body \\
\hline LSO ..... & Lateral superior olive \\
\hline MGB $\ldots . .$. & Medial geniculate body \\
\hline MI $\ldots . . . .$. & Monotonicity index \\
\hline MNTB ....... & Medial nucleus of the trapezoid body \\
\hline MSO ......... & Medial superior olive \\
\hline NMDA $\ldots . .$. & N-methyl-D-aspartate \\
\hline
\end{tabular}




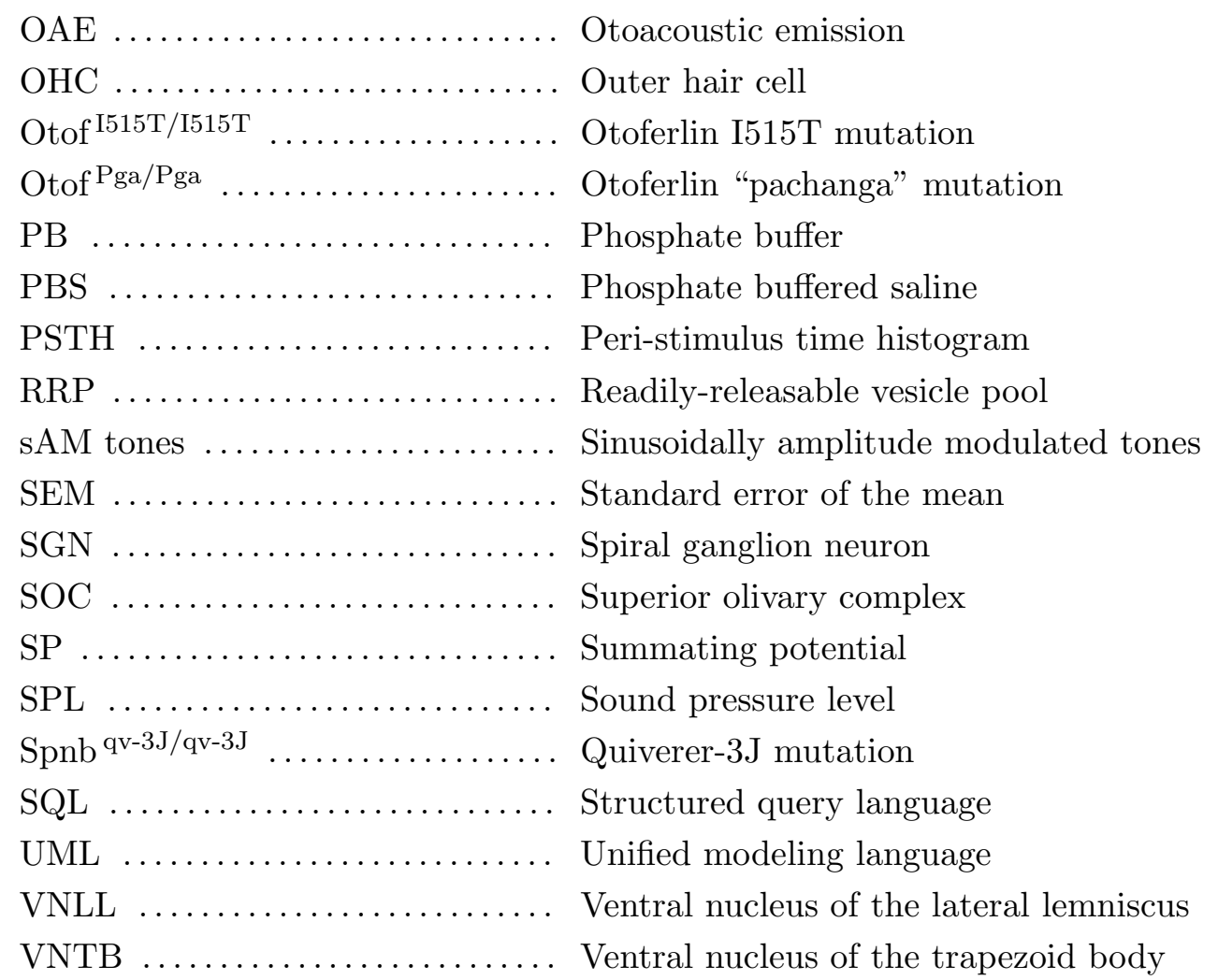




\section{Introduction}

Hearing is crucial for us, to perceive our environment, for the communication with other people and can be a great source of joy when it comes to appreciating music. Our hearing system can encode sound signals as faint as the sound of a bird's wing beat and loud as guitar riffs at a rock concert. It works on incredible short time scales and allows as to tell differences in sound down to a few microseconds.

One of the most important features of the hearing system is the ability to understand speech, which enables us to interact with other people and exchange information. However, communication with others is not only for exchanging information on an objective level. Subtle differences in the voice of other people tell us a lot about the emotions of this person. Speech does not only transport the information of what is said, but also on how it is said. The same sentence can have different meanings depending on the way it was said.

Hearing is however not only important for communication, but many people agree on music being an essential part of a high quality of life. Listening to music has the power of influencing our mood, making us feel good or sad, exciting us or calming us down.

The hearing system and brain have the task of combining all properties of sounds, including the reception and perception, but also the filtering and transformation of sound into electrical signals and their meaning.

People with hearing disorders lack an important sensory input to perceive their environment. Deafness is a strong impairment in the quality of live, especially in the communication with people. Although deaf people can communicate via sign language, most normal hearing persons do not understand this language. Many hearing deficits can be treated with the amplification of sounds via an external hearing aid. However, patients with congenital auditory neuropathies usually do not benefit from conventional hearing aids. Depending on the genetic cause of hearing loss, sometimes cochlear implants can be used to improve hearing function in those patients.

In this study we are following a systems physiology approach to study central sound encoding in the inferior colliculus (IC) of mouse models with auditory neuropathies and synaptopathies. These are disorders caused by impaired pre- or postsynaptic function of the inner hair cell ribbon synapse or neurons in the auditory pathway. We compare in vivo electrophysiological single unit recordings from the central nucleus of the IC (ICC) with before known recordings from the auditory nerve (AN) of mice with sound encoding deficits. 


\subsection{The mammalian ear}

The mammalian ear is responsible for capturing air-transmitted sound pressure waves and transfer them to an aqueous medium, where they can be detected by sensory cells. The sensory cells release neurotransmitters to elicit electrically transmittable action potentials in the nerve cells. The mammalian ear is composed of three functional parts, the outer-, middle- and inner ear. From the inner ear, acoustic information is transmitted as action potentials via the AN to the central auditory system, which is composed of numerous brainstem nuclei, each processing sounds in a different way until they are interpreted in the auditory cortex.

\subsubsection{Outer and middle ear}

The outer ear is the visible part of the hearing system, consisting of the pinna and the ear canal. The pinna efficiently captures sound waves and leads them into the ear canal. The ear canal ends at the tympanic membrane, which vibrates according to the incoming sounds. On the other side of the tympanic membrane is the middle ear cavity, an air filled cavern, containing the ossicles, comprised of malleus, incus and stapes. The base of the malleus is attached to the tympanic membrane, while the footplate of the stapes is attached to the oval window, an opening in the bony covering of the inner ear. With the help of this ossicle chain, the low-impedance airborne vibration is converted to higher-impedance vibration of the inner ear fluids (see Fig. 1.1 A).

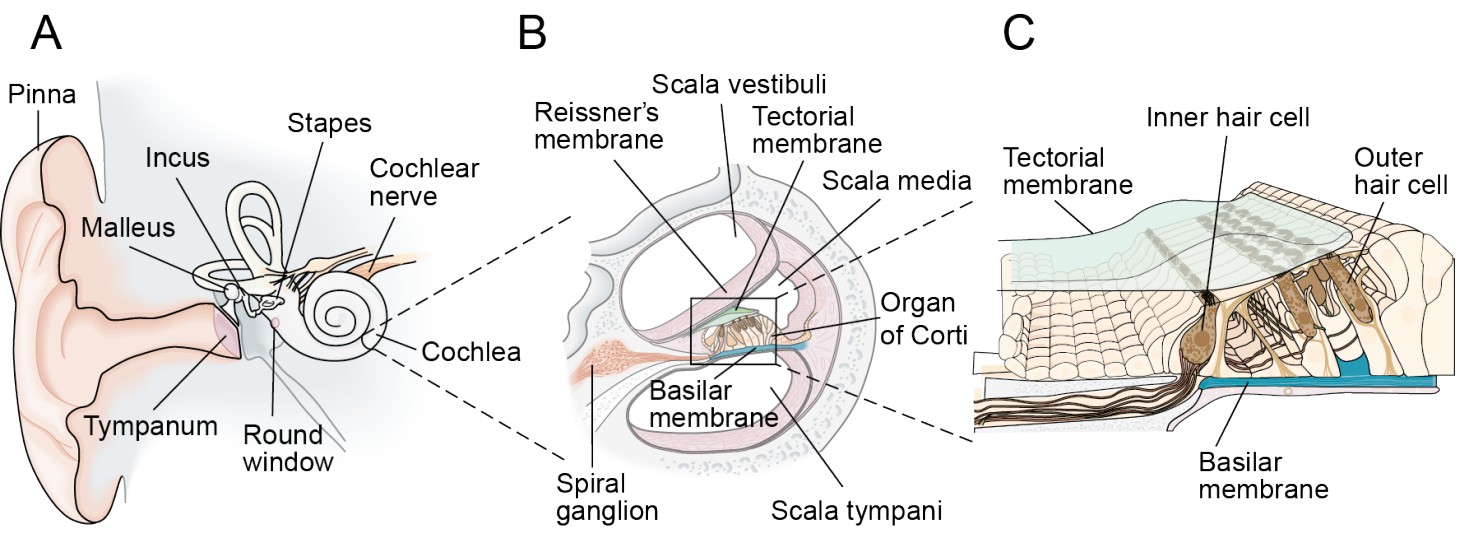

Figure 1.1.: Schematic view of the ear, cochlea and organ of Corti.

(A) Outer, middle and inner ear. (B) The bony structure of the cochlea is separated by Reissner's membrane and the basilar membrane into three compartments, the scala tympani, scala media and scala vestibuli. (C) The organ of Corti contains the sensory inner hair cells and the outer hair cells, which serve as amplifiers. Modified from Kandel et al. 2012 


\subsubsection{Inner ear and organ of Corti}

\section{Cochlea}

The inner ear is composed of the vestibular organ, responsible for the sense of balance, and a coiled bony structure, resembling a snail's shell, the cochlea. The inside of the cochlea is separated into three fluid-filled compartments, the scala vestibuli, which is separated by the middle ear through the oval window where the stapes is attached, the scala media and the scala tympani. The scala media is separated from the scala vestibuli by Reissner's membrane and from the scala tympany by the basilar membrane (see Fig. 1.1 B). Attached to the apical, scala media facing basilar membrane is the actual sensory organ, the organ of Corti, which detects sounds and transmits them to the spiral ganglion neurons (SGNs). The basilar membrane gradually varies in width, thickness and stiffness along the cochlea. Because of that, vibrations of the basilar membrane due to sound waves of a specific frequency will occur predominantly at a specific spot of the membrane. This leads to a frequency representation on the basilar membrane with high frequencies being represented at the base of the cochlea and low frequencies being represented at the apex. The frequency dependent organization of the cochlea is called tonotopy.

\section{Organ of Corti}

The organ of Corti sits on the basilar membrane and detects its vibrations. It is comprised of three rows of outer hair cells (OHCs), which function as enhancers and one row of sensory inner hair cells (IHCs). The hair cells are innervated from the bottom by the SGNs. OHCs are contacted by only a few afferent fibers but are innervated by many efferent fibers. In contrast, IHCs are contacted by many afferent fibers but only sparsely connected to efferent fibers and these connections mostly occur on the sensory axon terminals, the boutons of the SGNs (see Fig. 1.1 C).

\section{Sound generation}

When sound waves reach the inner ear, the basilar membrane starts vibrating selectively for a certain frequency at a specific area with the whole organ of Corti and all hair cells moving along with it. The cilia on top of the hair-cells are deflected, leading to the opening of mechano-gated ion channels and to the depolarization of the IHC. This depolarization causes calcium influx through voltage-gated calcium channels at the basal pole, which triggers fusion of synaptic vesicles at the ribbon-type active zones, releasing the neurotransmitter glutamate into the synaptic cleft (Nouvian et al. 2006). Upon stimulation, the OHCs have the unique feature of electromotility (Brownell 1990). They are able to mechanically elongate and shorten and thus actively enhance or dampen the movement of the basilar membrane, which leads to a sharpening of the response of the IHCs to a specific sound frequency by amplifying the sound-induced vibration of the basilar membrane (Zheng et al. 2000; Liberman et al. 2002; Fettiplace and Hackney 2006). 


\subsubsection{Inner hair cell ribbon synapse}

The IHCs contain specialized synapses forming contacts with the afferent SGNs in order to enable ultrafast and precise synaptic transmission (Moser et al. 2006). One IHC contains about 8-22 of these so-called ribbon synapses, at the basolateral membrane, each of them contacting a single SGN (Meyer et al. 2009). These numbers depend on the position along the basilar membrane and are species specific (mouse).

The name giving structure of the ribbon synapse, the ribbon is an electron dense structure, which contains large amounts of the protein ribeye and is anchored to the presynaptic membrane by the protein bassoon (see also chapter 1.5.1). It is surrounded by a halo of synaptic vesicles, tethered to the ribbon by short filaments. A subgroup of these vesicles, which are directly attached to the membrane likely correspond to the fusion competent vesicles composing the readily-releasable vesicle pool (RRP). The RRP can be released almost instantaneously within a few milliseconds of electrical stimulation into the synaptic cleft (Chakrabarti et al. 2018).

The ribbon synapse utilizes a set of proteins to manage exocytosis, which is quite different from those employed by conventional synapses. This specialization is thought to be required due to the ultrafast and permanent signaling in the auditory system. It has been shown that several common synaptic proteins like synapsin (Safieddine and Wenthold 1999), synaptotagmin (Reisinger et al. 2011) or complexins (Strenzke et al. 2009; Uthaiah and Hudspeth 2010) are absent in the ribbon synapse (Nouvian et al. 2011).

However, a few proteins have been identified which are present only in IHCs and are thought to take over the functions of the many missing common synaptic proteins. One important candidate to manage synaptic transmission in IHCs is the hair-cell specific multi C2-domain protein otoferlin, which will be further described in chapter 1.5.2.

Depolarization of the IHC leads to exocytosis of the neurotransmitter glutamate from the ribbon synapses, which is detected by $\alpha$-amino-3-hydroxy-5-methyl-4-isoxazolepropionic acid (AMPA) and N-methyl-D-aspartate (NMDA) receptors from the SGNs. Generated action potentials are initiated and transmitted via voltage-gated sodium channels, which are clustered to the axon initial segments (AIS) and nodes of Ranvier with the help of several proteins, among which is the protein spectrin (further described in chapter 1.5.4).

Exocytosis of synaptic vesicles is followed by fast endocytosis to be able to reform and replenish the vesicle pool, which has to occur fast enough to ensure the ultrafast synaptic reliability and temporal precision of the IHC ribbon synapses (von Gersdorff and Matthews 1994; Moser and Beutner 2000; Krächan et al. 2016).

\subsection{Spiral ganglion neurons (SGNs)}

The SGNs are the sole means of transmitting acoustic information from the inner ear to the auditory pathway and central nervous system. Therefore, all sounds are thus encoded in the timing and rates of spikes in the population of SGNs. Type-I SGNs form 
a single ribbon synapse with one IHC, while one IHC can form ribbon synapses with several SGNs. Glutamate release from the ribbon synapse leads to the generation of spikes in the SGNs. There is evidence that almost every release event evokes a single spike, unless the fiber is refractory (Siegel 1992), although it is under debate, if the release of neurotransmitter is caused by a single uniquantal release event (Rutherford et al. 2012; Chapochnikov et al. 2014) or if multiple vesicles synchronously release their neurotransmitter to the synaptic cleft (Glowatzki and Fuchs 2002; Grant et al. 2010).

A small proportion of unmyelinated type-II SGNs forms synapses with several OHCs. Little is known about these fibers as they are hard to record from and barely respond to sound stimuli (Heil and Peterson 2015).

\subsubsection{Sound encoding in spiral ganglion neurons}

Type-I SGNs possess different intrinsic and extrinsic characteristics like frequency selectivity, spontaneous rate and thresholds, as well as defined response patterns like spike-rate adaptation or phase-locking, which can slightly differ between species (Taberner 2005; Heil and Peterson 2015).

\section{Frequency selectivity}

Each SGN is most sensitive for one specific sound frequency, known as the characteristic frequency $(\mathrm{CF})$. This frequency selectivity is mainly defined by the position of the contacted IHC on the basilar membrane (Greenwood 1996). The threshold of a fiber is defined by the sound level where the spike rate of the fiber exceeds its spontaneous rate, while the $\mathrm{CF}$ is defined as the frequency with the highest spike rate at the lowest threshold. However, SGNs will not only fire in response to their CF, but also to neighboring frequency with a reduced spike rate. The resulting tuning curves of SGNs have a "V-shaped" form with asymmetrical areas of lateral suppression, reflecting the movements of the basilar membrane (Liberman 1982a; Taberner and Liberman 2005).

\section{Spontaneous rate and intensity coding}

SGNs have various spontaneous rates and can be divided into high-, medium- and lowspontaneous rate fibers (Liberman 1978). Pure tone thresholds and spontaneous rate in SGNs correlate. High-spontaneous rate fibers possess the lowest thresholds and lowspontaneous rate fibers possess the highest thresholds. Usually, one IHC is contacted by both high- and low-spontaneous rate fibers to be able to code for the whole range of possible sound pressure levels (SPLs). Thereby, high-spontaneous rate fibers primarily contact IHCs at the pillar side and low-spontaneous rate fibers preferentially contact the modiolar side of the IHCs (Liberman 1982b). Furthermore, ribbons on the pillar side seem to be smaller with less calcium channels and larger boutons than ribbons on the modiolar side (Merchan-Perez and Liberman 1996). It has been suggested that spontaneous rate is not an intrinsic property of SGNs, but a result of a homogeneous stochastic process of excitation due to transmitter release events (Heil et al. 2007). In addition to that, recent data by Shrestha et al. 2018 showed that the differentiation in 
high-, middle-, and low-spontaneous rate fibers occurs not only due to different inputs from the ribbon synapses, but also due to intrinsic molecular compositions of SGNs, which emerge during development.

The mean spike rate of single SGNs increases monotonically from the mean spontaneous rate with rising sound levels before it saturates at a certain sound intensity (Taberner and Liberman 2005). In more detail, three different forms of rate-level functions were described in SGNs, namely flat-saturating, saturating or straight (Sachs and Abbas 1974; Winter et al. 1990). All of them rising monotonically in spike rate with increasing sound level. To model the rate-level functions of SGNs, the mathematical approach of Sachs and Abbas 1974 was improved by Heil and Neubauer 2001 to generate a physiologically plausible model.

\section{Response patterns and Peri-stimulus time histograms}

The response pattern of SGNs to sustained pure tones varies over time and can be visualized by creating a peri- (or post-) stimulus time histogram (PSTH). This is generated by averaging the response to many repetitions of the same neuron to the same pure tone. The spike timing is counted in bins and divided by the number of repetitions and the bin width. The shape of the PSTH from SGNs usually shows a strong peak at the onset of the stimulus followed by an adaptation and decline until the response reaches a steady-state rate (called "primary-like" response; Galambos and Davis 1943; Taberner and Liberman 2005). At the offset of the tone, the response is often reduced to a level below the spontaneous rate until it gradually recovers. These changes in spike rate over time are thought to mainly arise from changes in synaptic transmission, such as depletion and replenishment of synaptic vesicles (Goutman and Glowatzki 2007). For instance, the sharp and strong onset peak likely arises from a depletion of the RRP (Moser and Beutner 2000),

\section{Phase-locking}

SGNs synchronize their firing pattern to the waveform of a pure tone, a feature that is known as phase-locking. However, phase-locking abilities of SNGs decrease with increasing frequencies, where the high-frequency limit of phase-locking is mainly determined by the time constant of the hair-cell membrane (Palmer and Russell 1986). In mice, phaselocking in a population of SGNs is possible to low frequency tones up to $4 \mathrm{kHz}$ (Taberner and Liberman 2005; Heil and Peterson 2017). However, pure tone phase-locking is of limited relevance to mice due to their high hearing frequency range between 2 and $70 \mathrm{kHz}$ (Heffner and Heffner 2007). Nevertheless, phase-locking to various frequency components of complex sounds like the envelope of sinusoidally amplitude modulated tones (sAM tones) with high carrier frequencies has been widely reported in mice (Khanna and Teich 1989; Joris and Yin 1992). To illustrate the precision of phase-locking, spike times from several phases can be counted as if they would have occurred in one phase and plotted as a period histogram. This histogram would have a sharp peak if strong phase-locking is present and a rather flat distribution if no phase-locking occurs. 


\subsection{The ascending auditory pathway}

When the auditory nerve enters the brainstem, it branches and innervates the different regions of the cochlear nucleus: the anteroventral cochlear nucleus (AVCN), the dorsal cochlear nucleus (DCN) and the posteroventral cochlear nucleus (PVCN). The tonotopic organization is maintained in all three areas, each containing different cells with different properties.

The AVCN is the first distributor of the ascending pathway where all the signals from the SGNs converge onto four different cell types and split up into four ascending auditory pathways (Oertel 1999). The four main cell types in the AVCN are spherical bushy cells (SBC), globular bushy cells (GBC), octopus cells and stellate cells. Each of those receive different inputs from the SGNs. In vivo they can be distinguished by their distinct spiking patterns in response to pure tone stimulation (Rhode et al. 1983).

Neuronal fibers from both ears partially cross sides and most nuclei in the auditory brainstem receive bilateral inputs. The different ascending pathways target different areas in the brain stem and midbrain, passing through the cochlear nuclei, the trapezoid body, superior olivary nuclei and the nuclei of the lateral lemniscus, ultimately bringing acoustic information mainly to the contralateral IC, which passes signals on to the thalamus and the auditory cortex (see Fig. 1.2). A tonotopic organization can be found in most areas of the auditory pathway.

\subsubsection{Anteroventral cochlear nucleus (AVCN)}

\section{Spherical bushy cells (SBCs)}

The SBCs receive inputs from few SGNs (Liberman 1991; Ryugo and Sento 1991) and resemble the "primary-like" PSTH shape of the SGNs, preserving and conveying the timing information. (Rhode et al. 1983). Most SBCs project to an isofrequency band in the contralateral medial superior olive (MSO, Smith et al. 1993).

\section{Globular bushy cells (GBCs)}

GBCs receive inputs from more SGNs and have a "primary-like-with-notch" PSTH with a sharp onset response followed by a break in firing, which is probably due to the neurons refractory period and a steady-state response (Rhode and Smith 1986; Rhode 2008). GBCs project mainly into the contralateral medial nucleus of the trapezoid body (MNTB) and partially in the ventral nucleus of the lateral lemniscus (VNLL) and the lateral superior olive (LSO, Spirou et al. 1990; Smith et al. 1991).

\section{Stellate cells}

Stellate cells from the AVCN convert the tonic SNG firing into a phasic response. They follow an intrinsic pacemaker, leading to a "chopping" response pattern (Rhode et al. 1983), which includes a precise and consistent timing of the onset responses (Rhode and Smith 1986). Furthermore, stellate cells are specialized to encode the envelope of 


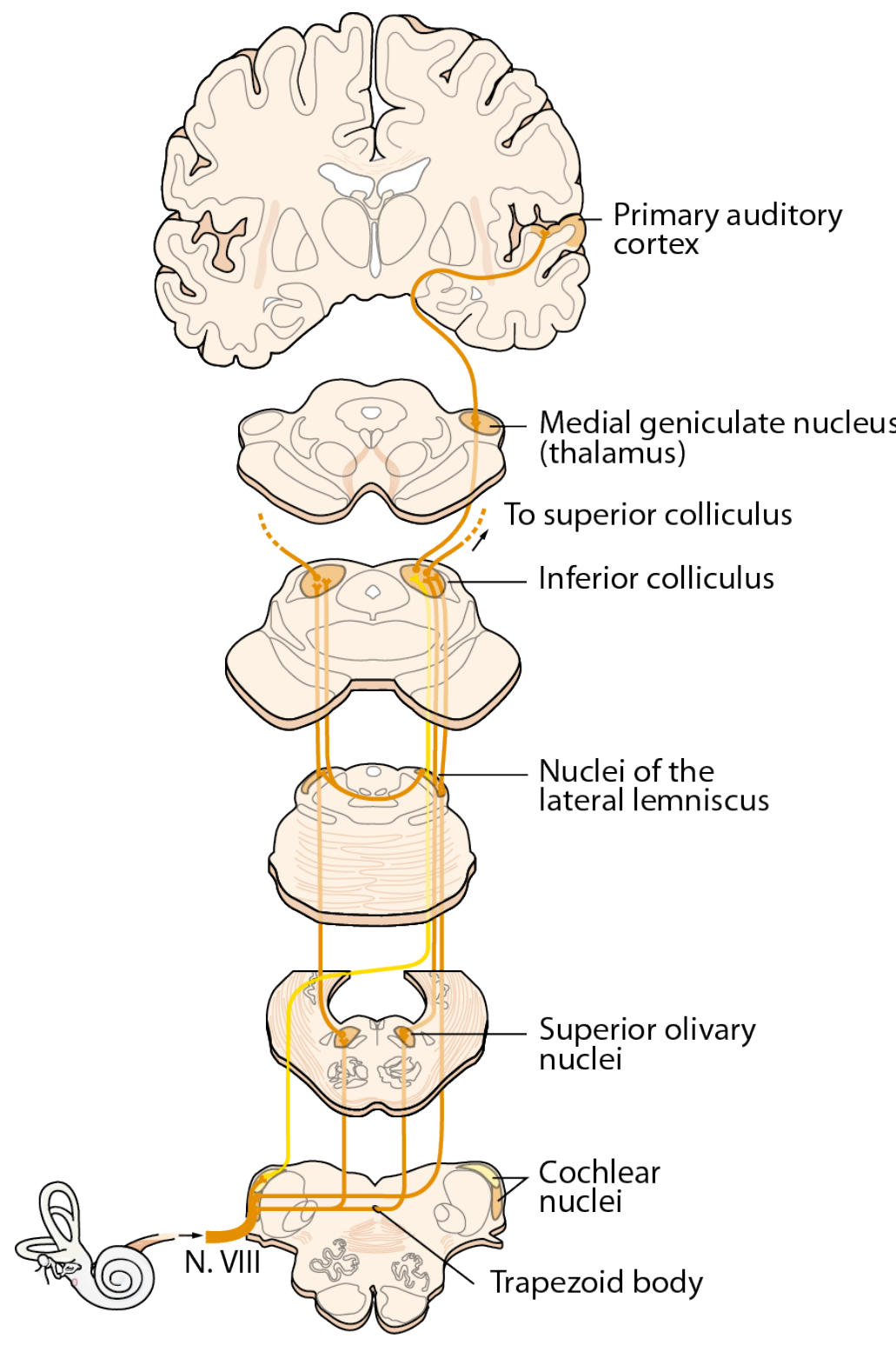

Figure 1.2.: Schematic view of the different ascending auditory pathways.

All SGNs project to the Cochlear nuclei, where the signal is split into four different ascending pathways. Bushy cells project mainly into the nuclei of the superior olivary complex on both sides. Stellate cells project mainly into the trapezoid body, the VNLL and the contralateral IC. Octopus cells project to the contralateral VNLL and to the periolivary nuclei. Nearly all pathways converge in the IC, from where they are passed on to the medial geniculate nucleus and the auditory cortex. Modified from Kandel et al. 2012

amplitude modulated (AM) sounds (Frisina et al. 1990) over a wide dynamic range (Smith and Rhode 1989). This feature makes them important candidates for the encoding of speech (Shannon et al. 1995). Stellate cells have long integration times from SGN inputs and project into the ventral nucleus of the trapezoid body (VNTB), the lateral nucleus of the trapezoid body (LNTB), the VNLL and to a great proportion to the contralateral IC (Oertel et al. 2011).

\section{Octopus cells}

Octopus cells receive inputs from a large number of auditory nerve fibers and encode coincident firing. Many SGNs have to respond synchronously to elicit a response in octopus cells (Golding et al. 1995; Oertel et al. 2000). They do not have considerable 
frequency tuning, but fire sharply timed action potentials with an "onset" pattern (Rhode et al. 1983). Octopus cells are specialized for the encoding of temporal fine structure and can phase-lock to frequencies up to $800 \mathrm{~Hz}$ where they respond with a single action potential to every phase (Langner 1992). Octopus cells mainly project to the contralateral VNLL and to the periolivary nuclei (Friauf and Ostwald 1988).

\subsubsection{Superior olivary complex (SOC)}

The superior olivary complex (SOC) is mainly responsible for sound localization by comparing interaural time differences (ITD) in the MSO (Fitzpatrick et al. 1997) and interaural level differences (ILD) in the LSO (Moore 1991; von Gersdorff and Borst 2002).

Sounds from one source arrive slightly shifted in time at both ears, if the source is not positioned dead center between the ears. Each ear transmits the sounds to both the ipsiand the contralateral MSO. Here, it has been suggested already in 1948 (Jeffress 1948), that cells are specialized for coincidence detection. The signals are transmitted through delay lines and will arrive at the same time from both ears specifically for each cell for a certain sound localization. More recent data by Stange-Marten et al. 2017 suggests additional differences in myelination to introduce delays. Further studies have shown a map of different ITDs or phase-delays for frequencies below $4 \mathrm{kHz}$ in the MSO (Yin and Chan 1990).

However, comparison of ITDs is only feasible for sounds with wavelengths that are longer than the width of the head. Therefore, a second mechanism is needed for sound localization of high frequency tones. Sounds arrive with different levels at both ears due to head shadowing effects and the LSO is able to compare the ILDs between ears. Neurons from the LSO receive excitatory inputs from the ipsilateral AVCN and inhibitory inputs from the contralateral AVCN and the ipsilateral trapezoid body. Sounds arriving at the ipsilateral side will cause strong excitation and weak inhibition. These differences are reflected in the spike rate of the neurons from the LSO, coding in this way for sound localization (Park et al. 2004).

\subsubsection{Ventral nucleus of the lateral lemniscus (VNLL)}

The VNLL resides higher up the auditory pathway and mainly receives inputs from the octopus cells. Consequently, the VNLL was proposed to play a role in spike timing analysis of auditory signals (Covey and Casseday 1991), duration and delay tuning (Casseday et al. 1994) and pattern recognition (Oertel and Wickesberg 2002). The VNLL is mainly composed of cells using glycine or GABA as neurotransmitter and is therefore thought to be an important source of inhibitory projections to the IC (Batra and Fitzpatrick 2002). 


\subsubsection{Inferior colliculus (IC) and its central nucleus (ICC)}

The IC is the central processing unit of the auditory system and the first integration center where almost all auditory information is conveyed, integrated and processed. All different ascending auditory pathways send projections to the IC (Beyerl 1978; Adams 1979; Brunso-Bechtold et al. 1981). It is composed of a central nucleus (ICC) and cortical areas. The cortex of the IC gets inputs mainly from the ICC (Saldaña and Merchán 2005) and descending inputs from the auditory cortex (Winer 2005; Ayala et al. 2015). Moreover, it is connected with the visual and somatosensory system, most likely playing a role in exchanging information between those systems (Yamauchi and Yamadori 1982; Itoh et al. 1984). In this thesis we will be only focusing on the ICC.

In the ICC, two different cell-types can be distinguished morphologically, namely bipolar or disc-shaped cells and multipolar or stellate cells (Oliver and Morest 1984, Meininger et al. 1986). However, unlike in the AVCN, where cell types can be classified into four categories, each having different properties, the morphology of the cells does not correlate with their axonal targeting, the neurotransmitters they produce, or their electrical properties.

Approximately $25 \%$ of IC neurons (ICNs) use $\gamma$-aminobutyric acid (GABA) or glutamate as neurotransmitters (Oliver et al. 1994; Merchán et al. 2005). The response properties of both cell types measured in vivo are similar regarding thresholds, response latencies, rate-level functions and frequency tuning, but glutamatergic neurons seem to be able to follow the envelope of sAM tones better than GABAergic neurons (Ono et al. 2017).

The major sources of ascending auditory inputs to the ICC are the contralateral cochlear nuclei (excitatory), which form synapses on both the disc-shaped and the stellate cells, the nuclei of the SOC (mainly excitatory), and the nuclei of the lateral lemniscus (inhibitory, Cant 2005; Schofield 2005). The major source of projections from the AVCN comes from stellate cells, while few if any globular cells project directly to the ICC and octopus cells do not terminate in the ICC (Beyerl 1978; Ryugo et al. 1981). Moreover, intrinsic and commissural connections exist in the IC and form an extensive network of local axon collateral, which may contribute a lot to neural processing within the IC (Oliver et al. 1991; Saldaña and Merchán 2005). The auditory cortex projects mainly into the IC cortex and only sparsely into the ICC (Winer et al. 1998).

Projections from the IC arrive partially in the ipsilateral superior colliculus, but the ICC mainly sends excitatory as well as inhibitory projections to the ipsilateral thalamus, more precisely the medial geniculate body (MGB), which is the relay to the auditory cortex (Winer et al. 1996; Peruzzi et al. 1997).

\subsubsection{Medial geniculate body (MGB)}

The MGB is part of the thalamocortical system and is an obligatory relay for all ascending auditory information, which proceed further to the auditory cortex. The MGBs main inputs originates from the IC, as well as few projections from lower centers of the auditory pathway that bypass the IC. Neurons from the MGB receive convergent inputs from 
spectrally and temporally separate pathways and are able to mediate the detection of specific spectral and temporal combinations of sounds. Numerous feedback and feedforward loops between the MGB and the cortex enable the processing of complex auditory tasks (Winer et al. 2005).

\subsubsection{Auditory cortex (AC)}

The final destination of all auditory signals is the auditory cortex where projections end in distinct areas. The most prominent projection runs from the ventral division of the MGB to the primary auditory cortex (A1). It is tonotopically organized and mirrors the organization in the cochlea. Responses in the auditory cortex encode numerous tasks and neurons are specialized for diverse sound features enabling the cortex to represent many independent variables of sound. Although auditory coding in the cortex is not understood very well, it is likely to be important for higher-order processing, for instance in terms of communication (Rauschecker and Tian 2000; Schreiner and Winer 2007).

\subsection{Sound encoding in the inferior colliculus}

As mentioned before, the ICC is the main integration center of the auditory pathway to which most nuclei send projections. This makes the interpretation of responses from ICNs difficult, because it is unknown which inputs a single neuron receives or which task a single neuron fulfills. Still, some common characteristics of ICNs have been identified in terms of frequency and intensity coding or response patterns of spiking.

\subsubsection{Frequency selectivity}

The ICC is tonotopically organized with low frequencies being represented dorsolaterally and high frequencies ventromedially (Merzenich and Reid 1974). The tonotopic organization in the ICC is less continuous but a series of iso-frequency bands. These bands are generated by the disc-shaped cells of the ICC, which form fibrodendritic laminae, each coding a specific frequency (Stiebler and Ehret 1985; Schreiner and Langner 1997; Egorova et al. 2006).

Responses of single ICNs have a high diversity when looking at the frequency response areas (FRAs), including broadly and narrowly tuned units. This reflects the presence of inhibitory and facilitatory circuits in the IC, shaping the FRAs (Palmer et al. 2013).

FRA shapes that were frequently encountered have been classified by Ramachandran et al. 1999 into different categories. Hereby so-called "V-shaped" neurons are broadly tuned and have v-shaped excitatory areas. "I-shaped" neurons have a narrow tuning, due to flanking by inhibitory regions and "O-shaped" neurons have strong inhibitory areas leaving only a small excitatory field, specific for a certain $\mathrm{CF}$ and sound level. Neurons with V- I- and O-shaped FRAs could be also identified during the recordings for this thesis and this classification was adapted for our purposes (see chapter 3.7.1). 


\subsubsection{Intensity coding}

Rate-level functions in response to tone bursts in the ICC have more complex shapes than those in the SGNs. They can appear in monotonic, plateau, non-monotonic or complex shapes (Ehret and Merzenich 1988a). Moreover, sound intensities may influence neural responses in changing spectral filter width, altering neural response strength in terms of firing rate, or modifying temporal response parameters such as latency and precision of action potential timing.

Single unit thresholds in response to pure tones are in some units even lower than the behavioral thresholds, although when averaging all units the threshold resembles the behavioral curve in most of the frequency range (Ehret and Moffat 1985; Egorova et al. 2001). However, this may vary a lot due to the physiological state and anesthesia of the animal (Zurita et al. 1994).

\subsubsection{Response patterns}

Studies involving whole cell patch clamp recordings found a variety of different discharge patterns like buildup, accelerating, burst-onset, burst-sustained, sustained or accommodating (Tan et al. 2007), but unlike the responses in the AVCN, these different patterns could not be matched to different cell types in the ICC (Peruzzi et al. 2000).

In vivo a broad variety of responses was shown as well (Willott and Urban 1978). Less than half of the units in this study had spontaneous spiking. The most abundant responses included phasic onset responses and sustained excitatory responses, but many other types like "chopper", "pauser" or "off" responses, as well as sustained inhibition were also encountered. A further characterization was done by Ehret and Moffat 1985, showing tonic, "primary-like" responses (comparable to SGN responses), phasic, phasic with some tonic activity, "pauser", long latency and spontaneous activity inhibited response patterns.

\subsubsection{Phase-locking}

At the stage of the IC, temporal modulation can be represented by synchronized responses (phase-locking) or non-synchronized responses (spike rate code). Most studies on phaselocking in the IC are carried out using stimulation with AM tones with the carrier frequency at CF. Neurons in the ICC are generally able to phase-lock to the envelope of these tones, but as upper cutoff frequencies for phase-locking decrease systematically along the ascending auditory pathway (Joris et al. 2004), phase-locking in the ICC is only possible at low modulation frequencies. In addition to that, anesthesia has an influence and depresses phase-locking, especially in higher brain centers (Langner et al. 2002). Synchronous responses of single ICNs can have either band-pass characteristics, coding specifically for a small range of modulation frequencies or low-pass characteristics.

It has been suggested, that a conversion of the phase-locking code, represented by synchronized responses (spike timing code) into a rate- or space-based representation might 
take place in the IC (Joris et al. 2004). Looking at the spike rate in dependency to the modulation frequency, the best ICNs are able to respond specifically up to $600 \mathrm{~Hz}$ modulation frequency (Langner et al. 2002). Moreover, responses to AM tones are not only coded by modulation frequency in the ICC, but also according to the duration and duty cycle of the stimulation (Krebs et al. 2008).

Periodicity coding seems to be an important feature which is processed in the ICC. On top of the tonotopic organization in laminae, a certain, although less pronounced, organization of periodicities seems to be present in the ICC. Neurons responding to certain periodicities of sAM sounds seem to appear in columns (in cats, Schreiner and Langner 1997) or in clusters (in gerbils, Schnupp et al. 2015).

\subsubsection{Sound localization}

The ICC gets inputs bilaterally from the SOC and plays a major role in sound localization. Many neurons in the IC have been shown to be sensitive for difference in interaural time, level or phase (Grothe et al. 2010). However, in this thesis we do not investigate sound localization, as we used bilateral stimulation delivered by a free-field loudspeaker, placed in front of the animal's head.

\subsection{Single unit recordings from mice with auditory neuropathies and synaptopathies}

Auditory neuropathies are a range of hearing disorders, which are clinically described with normal or near-normal cochlear amplification by OHCs, but impaired sound encoding at the level of the IHC ribbon synapse (called auditory synaptopathy), the auditory nerve or sometimes at higher levels of the auditory pathway (Moser and Starr 2016). Often patients have normal pure tone thresholds in an audiogram and normal otoacoustic emissions (OAEs) and cochlear microphonics, but impaired or absent auditory brainstem responses (ABRs). ABR waves reflect synchronized responses of the different stations of the auditory pathway, providing a measure of signal processing in the whole system (Melcher, Guinan, et al. 1996, Melcher, Knudson, et al. 1996, Melcher and Kiang 1996). The grade of the hearing disorder can range from deafness, where patients gain no useful information from auditory signals to mild forms where patients have difficulties understanding speech in noise. Patients with auditory neuropathies rarely benefit from conventional hearing aids.

This chapter summarizes published data of the mouse lines used in this study, which have different mutations leading to different forms of auditory synaptopathies and neuropathies. All these mouse lines have already been characterized by recording ABRs and in vivo electrophysiological recordings from the AN. The different genetic backgrounds of the mutations and the previously obtained data are presented in this chapter.

The mouse lines used in this study possess mutations in the IHC specific protein otoferlin, which in the one case (otoferlin Ile515Thr) lead to a mild hearing impairment with 
near normal pure tone thresholds but increased adaptation to continuous sounds and in the other case (otoferlin pachanga) to deafness with almost, but not completely abolished vesicle exocytosis. Furthermore, we were investigating a bassoon mutant mouse, leading to a reduced number of synaptic ribbons, which are not properly anchored to the active zone, resulting in reduced SGN spike rates. The fourth mouse line used in this study (quiverer-3J) has a mutation in all neurons, leading to an overall reduced spike propagation velocity.

\subsubsection{Bsn ${ }^{\Delta E x 4 / 5}$ mice}

Bassoon is a scaffold protein, that plays a role at synaptic active zones in the assembly of proteins important for synaptic vesicle docking and fusion (Fejtova and Gundelfinger 2006). Furthermore, it is thought to anchor the ribbon to the presynaptic membrane and is implicated in the localization of voltage gated calcium channels (Gundelfinger et al. 2015). To study the role of bassoon, a bassoon mouse with a partial deletion was generated by excision of Exon 4 and 5, abolishing the expression of functional bassoon protein (Bsn ${ }^{\Delta E x 4 / 5}$ mouse line, Altrock et al. 2003).

In neuronal cultures, this loss of bassoon causes reduction in synaptic transmission by inactivation of a significant fraction of glutamatergic synapses. Vesicles are clustered and docked normally but unable to fuse. Loss of bassoon is not essential for synapse formation but plays a role in regulated neurotransmitter release. Moreover, due to these deficits, the mice suffer from epileptic seizures (Altrock et al. 2003).
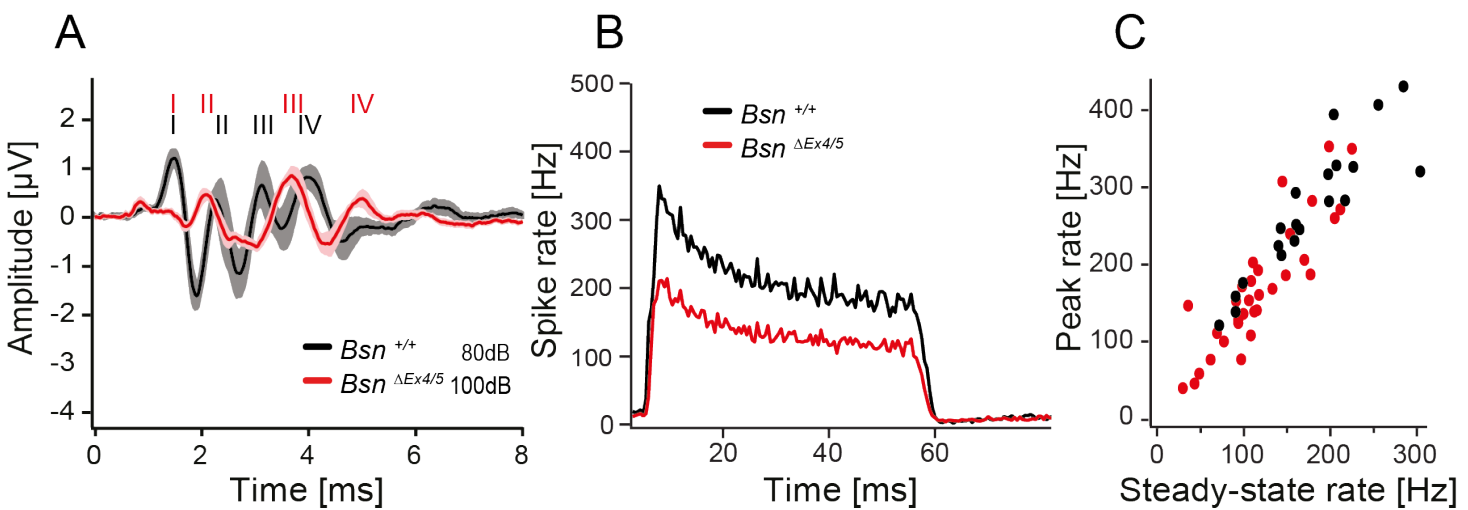

Figure 1.3.: Auditory brainstem responses and SGN recordings of $B s n^{\Delta E x 4 / 5}$ mice. Adapted from Jing et al. 2013.

(A) Grand averages of ABR waveform \pm SEM in response to $80 \mathrm{~dB}$ (wildtype, $\mathrm{n}=6$ ) and $100 \mathrm{~dB}$ (mutant, $\mathrm{n}=6$ ) click stimulation in $B s n^{\Delta E x_{4} / 5}$ mice. ABR wave I amplitude is strongly reduced.

(B) Averaged PSTH shows a preserved activation pattern, but reduced spike rates in SGNs from $B s{ }^{\Delta E x 4 / 5}(\mathrm{n}=31)$ and wildtype mice $(\mathrm{n}=23)$. (C) Peak rate as well as steady-state rates were reduced in SGNs from $B s n^{\Delta E x 4 / 5}$ mice.

In the IHC ribbon synapse of these mice, most ribbons were lost or not anchored to the active zone (Khimich et al. 2005; Buran et al. 2010). The general hearing function was tested by recording ABRs (Jing et al. 2013). Bsn ${ }^{\Delta E x 4 / 5}$ mice did not have a significant 
ABR amplitudes at $80 \mathrm{~dB}$ SPL. At $100 \mathrm{~dB}$ SPL the waveform, especially wave I was reduced and distorted (see Fig. 1.3 A).

Electrophysiological recordings from auditory nerve fibers revealed normal thresholds and dynamic range as well as normal adaptation and phase-locking but reduced spontaneous and sound-evoked discharge rates and an increased variance of first spike latencies (Buran etal. 2010; Jing etal. 2013, see Fig. 1.3 B-C). Similar results have been observed in a bassoon gene trap mutant with minimal residual bassoon expression (Jing et al. 2013).

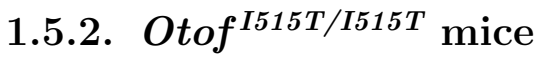

Otoferlin is an IHC specific multi-C2-domain protein essential for hearing. In otoferlin knock-out mice, depolarization induced exocytosis of synaptic vesicles is almost completely abolished (Roux et al. 2006). It has been suggested that otoferlin functions as a calcium sensor at the vestibular hair cell synapse (Dulon et al. 2009) and the IHC ribbon synapse, where it is thought to also act as a priming factor (Roux et al. 2006; Johnson and Chapman 2010; Pangrsic et al. 2010). Furthermore, the role of otoferlin as an important key player in IHC ribbon synapse exocytosis is indicated by Vogl et al. 2015, showing that otoferlin is enriched in the membrane of the IHC active zone, where it also plays a role in vesicular tethering. Additionally, otoferlin has been shown to not only influence vesicle exocytosis, but also endocytosis as it interacts with the adapter protein AP-2, an essential component of clathrin-mediated endocytosis (Duncker et al. 2013; Jung et al. 2015). In accordance with this, further roles of otoferlin have been indicated in vesicle reformation and replenishment (Strenzke et al. 2016; Michalski et al. 2017).

In human patients, most mutations in otoferlin lead to profound prelingual deafness, DFNB9 (Yasunaga et al. 1999; Choi et al. 2009). However, a few specific point mutations have been shown to cause only mild to moderate hearing impairments (Pangršič et al. 2012; Strenzke et al. 2016). In this thesis we're studying the Ile515Thr point mutation, a missense mutation in the $\mathrm{C} 2 \mathrm{C}$ domain of otoferlin, which leads to hearing impairments with abnormal adaptation to continuous tones, clinically described as auditory fatigue (Wynne et al. 2013). Furthermore, this mutation belongs to the group of temperaturesensitive mutations, where residual hearing is strongly temperature dependent and patients turn reversibly deaf upon elevation of the body temperature (Marlin et al. 2010; Zhang etal. 2016). The generated mouse line mimics the human phenotype, although the temperature sensitivity was only partly reproduced in mice (Strenzke et al. 2016).

Hair cell morphology and function in the Otof ${ }^{I 515 T / I 515 T}$ mouse line has been examined in detail by electron and immunofluorescence microscopy, as well as by cell and systems physiology regarding IHCs and SGNs, which will be presented in short hereafter (Strenzke et al. 2016).

Hearing function was assessed by measuring ABRs (see Fig. 1.4). While at 4 weeks of age only ABR wave I was reduced, at 8-25 weeks of age all ABR amplitudes were drastically reduced, with wave I being completely absent while wave IV was reduced, but better preserved. ABR thresholds were highly elevated. Patch-clamp recordings from IHCs of 
Otof ${ }^{I 515 T / I 515 T}$ mice showed that the sustained vesicle exocytosis is impaired, but the fast component of exocytosis is normal.

A

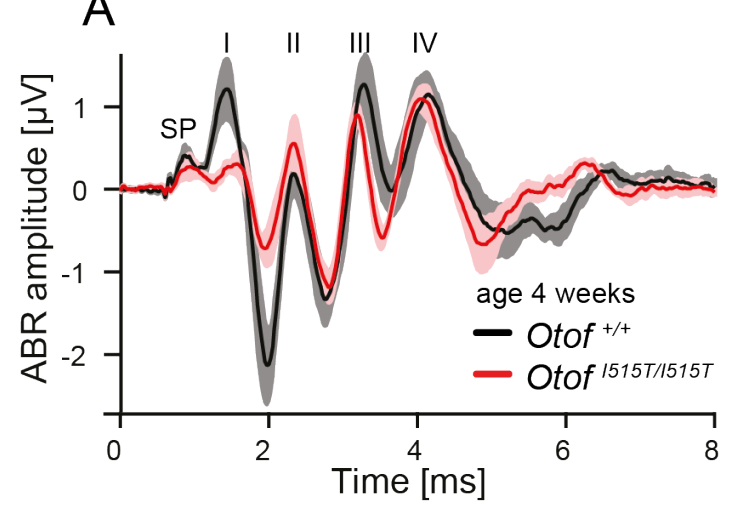

B

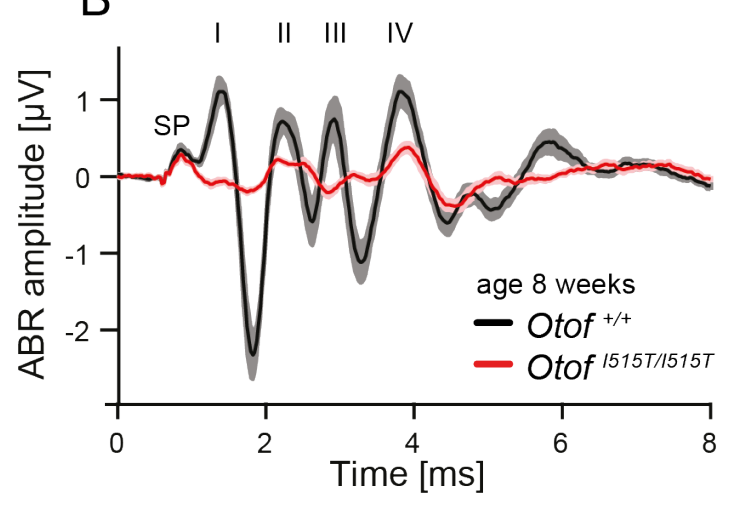

Figure 1.4.: Auditory brainstem responses of Otof ${ }^{1515 T / I 515 T}$ mice. Adapted from Strenzke et al. 2016.

Grand averages of $\mathrm{ABR}$ waveform \pm SEM in response to $80 \mathrm{~dB}$ click stimulation in Otof ${ }^{1515 T / 1515 T}$ mice at the age of (A) 4 weeks (mutant $\mathrm{n}=5$, wildtype $\mathrm{n}=5$ ) and (B) 8 weeks (mutant $\mathrm{n}=8$, wildtype $\mathrm{n}=7$ ). The summating potential (small elevation preceding wave $\mathrm{I}$ ) is intact in Otof ${ }^{1515 T / 1515 T}$ mice. (A) At 4 weeks of age, the amplitude of ABR wave I is greatly reduced while other waves are not impaired. (B) Otof ${ }^{1515 T / I 515 T}$ mice at 8 weeks of age have drastically reduced ABR amplitudes. ABR wave I is almost completely gone while subsequent waves are better preserved, but still poor.

Moreover, in vivo single unit recordings from the AN were recorded from Otof ${ }^{I 515 T / I 515 T}$ mice. Here it was shown, that spontaneous rates were normal, but sound evoked spike rates showed a decrease in steady-state rate with an impaired time course of adaptation and a trend towards decreased peak rates, especially prominent when stimulating with higher frequencies (see Fig. 1.5 A-D). First spike latencies were slightly more jittered in SGNs of Otof ${ }^{I 515 T / I 515 T}$ mice compared to Otof ${ }^{+/+}$littermates. Rate-level functions were shallower with a reduction in maximal steepness, but of normal dynamic range. Phase-locking in response to AM tones with a modulation frequency of $500 \mathrm{~Hz}$ seemed to be impaired, shown by a trend towards reduced maximal synchronization indices (see Fig. 1.5 E). Furthermore, in a forward masking paired-tone stimulus, where a $100 \mathrm{~ms}$ masker tone was played, followed by a variable silent interval and a $15 \mathrm{~ms}$ probe tone, an increased depression of spiking was observed with a delayed recovery from forward masking (see Fig. $1.5 \mathrm{~F}$ ). A behavioral experiment revealed a deficit in coding silent gaps in noise in Otof ${ }^{1515 T / I 515 T}$ mice compared to Otof $+/+$ littermates (see Fig. 1.5 G). Immunohistochemical staining of IHCs from Otof ${ }^{I 515 T / I 515 T}$ mice revealed reduced levels of otoferlin at the plasma membranes. This might be the major cause of the observations presented before. 
A

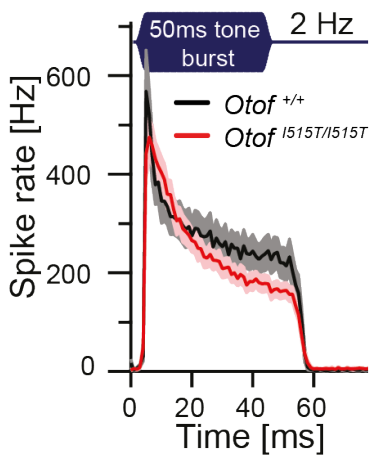

B

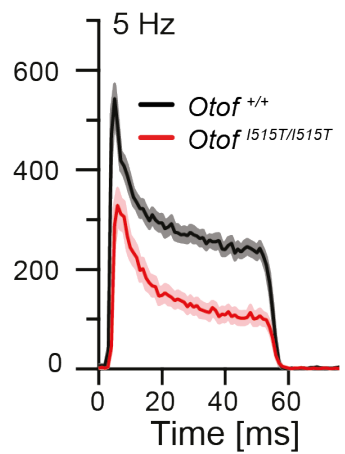

C

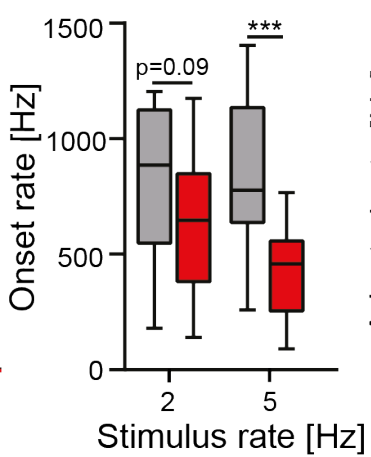

D

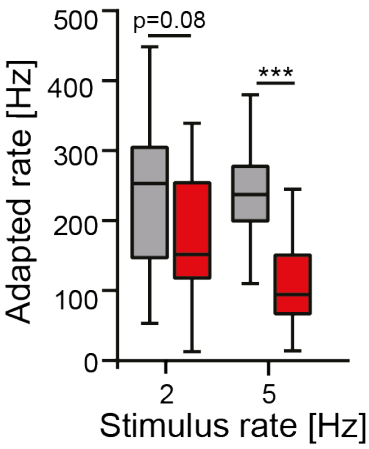

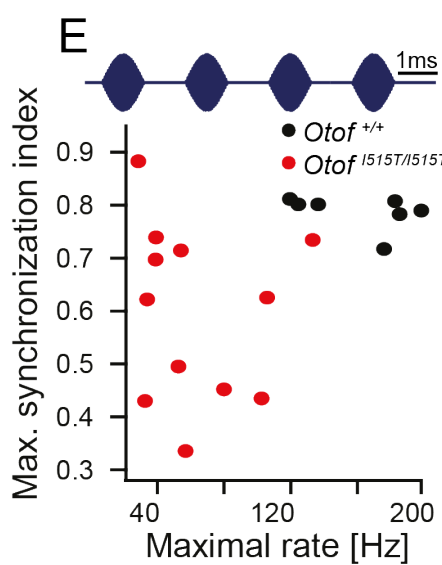
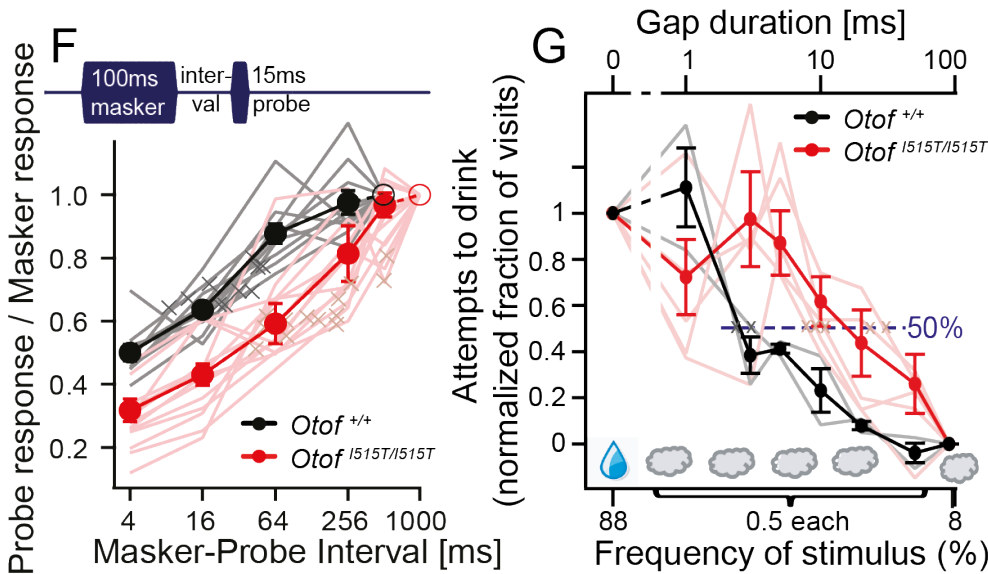

Figure 1.5.: Responses from SGNs of Otof ${ }^{I 515 T / I 515 T}$ mice. Adapted from Strenzke et al. 2016.

(A-B) Averaged PSTHs \pm SEM from Otof $1515 T / I 515 T$ SGNs $(\mathrm{n}=25-32)$ and Otof $+/+$ SGNs $(\mathrm{n}=13-27)$ to stimulation with $50 \mathrm{~ms}$ tone bursts at $\mathrm{CF}, 30 \mathrm{~dB}$ above threshold at stimulus rates of (A) $2 \mathrm{~Hz}$ and (B) $5 \mathrm{~Hz}$. (C-D) Onset rates tend to be smaller for stimulation at (C) $2 \mathrm{~Hz}$ and (D) $5 \mathrm{~Hz}$, while steady-state rates are reduced in both stimulation conditions. Furthermore, time course of adaptation is increased in Otof ${ }^{I 515 T / I 515 T}$ mice. (E) Phase-locking to transposed tone showed a trend towards worse temporal precision, accessed by the maximal synchronization index $(\mathrm{p}=0.09)$. ( $\mathrm{F})$ Forward masking revealed enhanced RRP depletion after stimulation and a slowed time course of recovery. (G) Otof ${ }^{I 515 T / I 515 T}$ mice $(\mathrm{n}=5)$ performed worse than Otof ${ }^{+/+}$ mice $(n=2)$ for shorter gap durations in a behavioral task where mice had to learn to drink water when continuous noise was present but avoided drinking when the noise was interrupted by silent gaps.

\subsubsection{Otof ${ }^{P g a / P g a}$ mice}

The Otof Pga/Pga (or "pachanga") mutation is a mutation in otoferlin, more precisely an Asp1767Gly substitution in the C2F-domain of otoferlin. However, the Otof Pga/Pga mutation leads to a more severe hearing impairment than the Otof ${ }^{I 515 T / I 515 T}$ mutation. The pachanga mutation has been identified by a forward genetics screen in mice to identify deafness genes and Otof ${ }^{P g a / P g a}$ mice are behaviorally deaf as they show no acoustic startle response (Schwander etal. 2007). Synaptic vesicle exocytosis is almost 
completely abolished (Pangrsic et al. 2010) and synaptic vesicles seem to accumulate at the docked stage without being able to properly fuse with the membrane (Chakrabarti et al. 2018). IHC ribbon synapse function and auditory coding in the SGNs have been studied before and will be presented here (Pangrsic et al. 2010):

ABR waves from Otof ${ }^{P g a / P g a}$ mice are completely absent. However, a small elevation before wave I can be seen, indicating a normal summating potential (SP) in the IHCs (see Fig. 1.6 A). In line with this, normal SPs can be seen in the electrocochleography, but compound action potentials (CAPs) from the SGNs are completely absent (see Fig. 1.6 B).
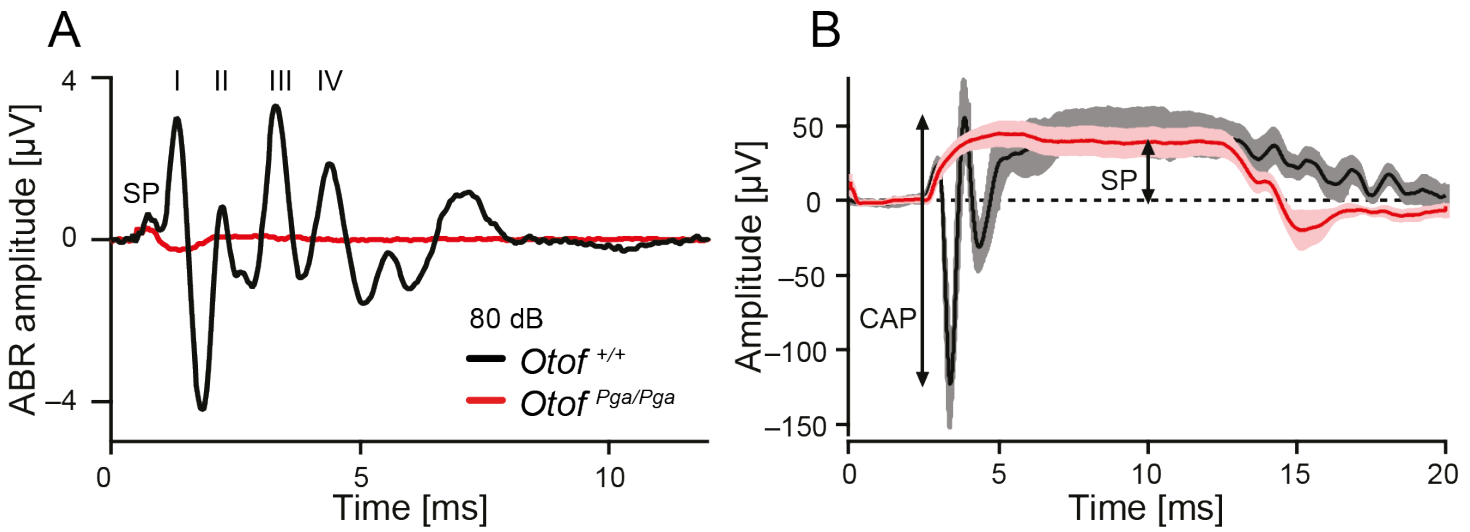

Figure 1.6.: Auditory brainstem responses of Otof $^{P g a / P g a}$ mice. Adapted from Pangrsic et al. 2010.

(A) No ABRs were elicited at $80 \mathrm{~dB}$ click stimuli in 2-4-week-old Otof Pga/Pga mice, while the summating potential (SP) is intact. (B) Averages \pm SEM of low pass-filtered electrocochleography traces in response to $12-\mathrm{ms}, 90 \mathrm{~dB}, 12 \mathrm{kHz}$ tone bursts. Normal summating potentials are evoked in Otof ${ }^{P g a / P g a}$ mice, but no spiral ganglion compound action potential (CAP) can be seen.

Patch-clamp experiments revealed a normal vesicle fusion, but exocytosis is strongly fatiguing. Vesicle replenishment is impaired and RRP refilling is slowed in Otof Pga/Pga IHCs, but docking and endocytic membrane retrieval are unaltered. Otoferlin protein levels are strongly reduced and only present at the membranes in small amounts (Strenzke et al. 2016). Single unit recording from the region of the AVCN and AN reveal strongly depressed sound evoked spiking with elevated thresholds in the SGNs. It was shown to be difficult to find any sound responsive neurons, which were impossible to classify. Spiking often stopped during the first trials. At $10 \mathrm{~Hz}$ stimulation rate Otof ${ }^{+/+}$SGNs fire at unchanged high rates, while Otof ${ }^{P g a / P g a}$ SGNs barely respond at all (see Fig. 1.7 A). However, by increasing the recovery time through slower stimulation, some sound evoked spiking could be elicited also in Otof ${ }^{P g a / P g a}$ mice (see Fig. 1.7 B-C). 

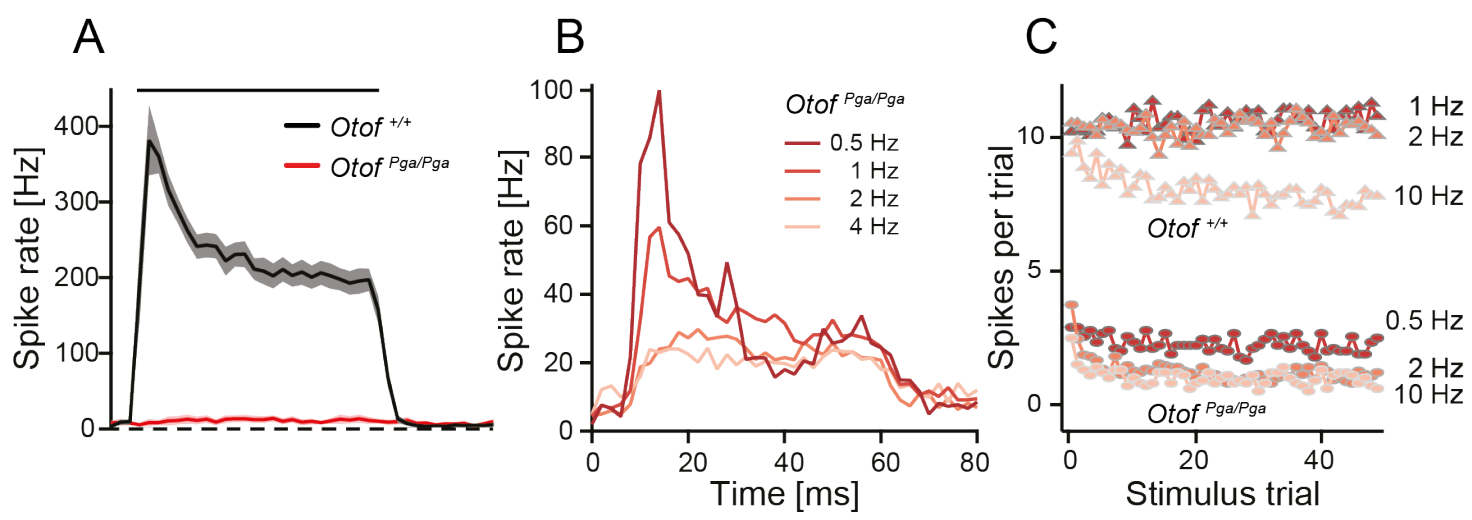

Figure 1.7.: Responses from SGNs of Otof ${ }^{P g a / P g a}$ mice. Adapted from Pangrsic et al. 2010.

(A) Mean PSTH \pm SEM of SGNs from Otof ${ }^{+/+}$mice $(n=15)$ and sound-responsive neurons from the region of the cochlear nucleus in Otof ${ }^{P g a / P g a}$ mice $(\mathrm{n}=10)$, stimulated with tone bursts at $\mathrm{CF}$, $30 \mathrm{~dB}$ above threshold at $10 \mathrm{~Hz}$ repetition rate. Hardly any responses are detected in Otof Pga/Pga mice. (B) PSTHs of Otof ${ }^{\text {Pga/Pga }}$ units stimulated at different rates $(\mathrm{n}=8-13)$. The higher the stimulation the fewer spikes are elicited. (C) The number of sound-evoked spikes during the first 50 trials of stimulation is strongly reduced in Otof ${ }^{P g a / P g a}$ mice $(\mathrm{n}=15)$ compared to wildtype mice $(\mathrm{n}=15)$. Spike rate drops in the first few trials at 2 and $10 \mathrm{~Hz}$.

\subsection{4. $S p n b^{q v-3 J / q v-3 J}$ mice}

Action potential generation and saltatory conduction in myelinated nerve fibers is only possible due to clustering of voltage gated sodium channels at the nodes of Ranvier and the AIS. The organization of the AIS is a complicated process largely dependent on the protein ankyrin-G, which has been shown to be a master scaffolding protein, responsible for the recruitment of many proteins, including voltage gated sodium and potassium channels (Huang and Rasband 2018). Ankyrin-G binds to the actin cytoskeleton via $\beta 4-$ spectrin, which was identified as the specific spectrin found at AIS and nodes of Ranvier (Berghs et al. 2000; Jenkins and Bennett 2001; Komada and Soriano 2002).

$\beta 4$-spectrin is a scaffolding protein regulating nodal structural integrity and molecular organization and occurs in six different isoforms $(\Sigma 1-\Sigma 6 \beta 4)$. Absence of $\Sigma 1 \beta 4$-spectrin leads to destabilized nodes of Ranvier and AIS (Lacas-Gervais et al. 2004) and disturbs sodium channel clustering (Komada and Soriano 2002). Nodes are increased in diameter and ankyrin-G clusters appear fragmented, consistent with a decreased nerve conductivity (Lacas-Gervais et al. 2004).

The "quivering" mutation, that arouse spontaneously in 1953 (Yoon and Les 1957) has been shown to be caused by truncating mutations in $\beta 4$-spectrin, leading to auditory and motor neuropathies with ataxia (Parkinson et al. 2001). The different quivering variants $\left(\mathrm{qv}^{\mathrm{J}}, \mathrm{qv}^{2 \mathrm{~J}}, \mathrm{qv}^{3 \mathrm{~J}}, \mathrm{qv}^{4 \mathrm{~J}}, \mathrm{qv}^{\mathrm{Ind}}\right.$ and $\left.\mathrm{qv}^{\mathrm{Ind} 2 \mathrm{~J}}\right)$ have different lengths of truncation, which correlate with the severity of the phenotype. In "quivering" mice it has been demonstrated as well, that sodium channel distribution is disrupted in the sensory epithelium and ganglion (Hossain et al. 2005). 
The quivering-3J mice (Spnb ${ }^{q v-3 J / q v-3 J}$ mice) we are using in this study have moderate sensory- and motor impairments with locomotor instabilities, quivering, hind limp paralysis as well as hearing impairments. It has a truncated proline-rich "specific" domain and lack the pleckstrin homology domain. Ion channel clustering is impaired, nodes of Ranvier have been shown to be broader in $S p n b^{q v-3 J / q v-3 J}$ mice and axon shape is altered (Yang 2004).

The auditory function of $S p n b^{q v-3 J / q v-3 J}$ mice has been further characterized recently and will be presented hereafter (Yamanbaeva 2017):

ABRs were recorded from $S p n b^{q v-3 J / q v-3 J}$ mice and wildtype littermates at the age between 7 and 15 weeks. Recordings revealed elevated thresholds of about $20 \mathrm{~dB}$ (see Fig. 1.8 A). The ABR waveform is broadened and delayed, but ABR amplitudes are normal at least for wave I and II (see Fig. 1.8 B). ABR wave latencies are increased and progressively increase along the brainstem (see Fig. 1.8 C).
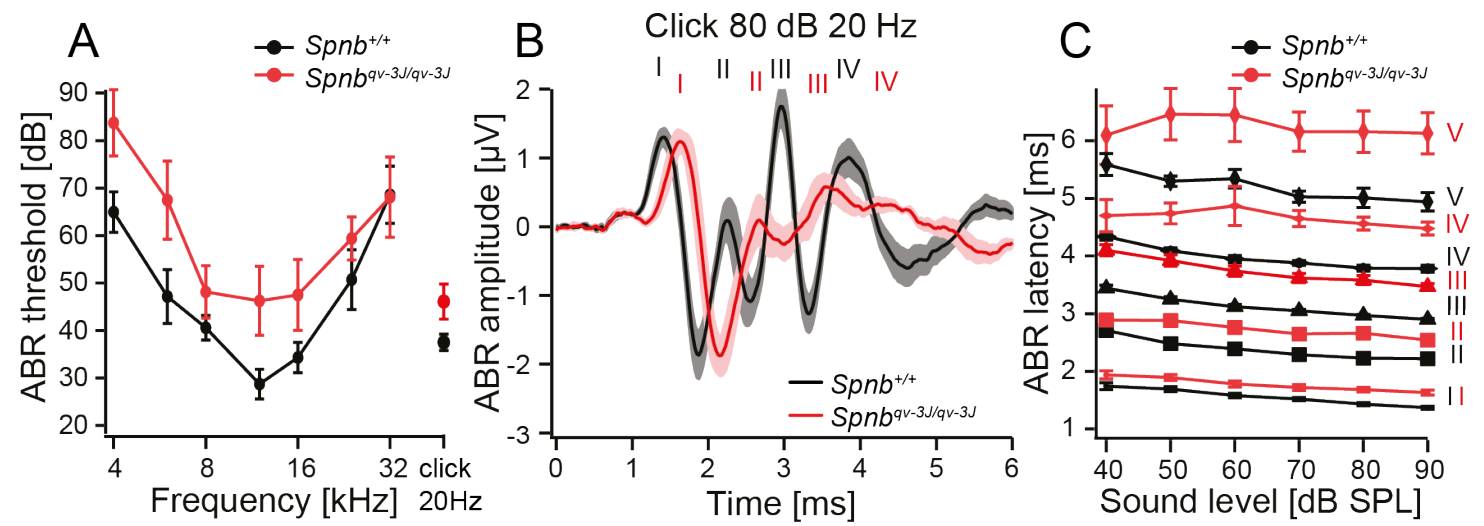

Figure 1.8.: Auditory brainstem responses of $S p n b^{q v-3 J / q v-3 J}$ mice. Adapted from Yamanbaeva 2017.

(A) ABR thresholds are elevated in $S p n b^{q v-3 J / q v-3 J}$ mice $(\mathrm{n}=8)$ compared to $S p n b^{+/+}$littermates $(\mathrm{n}=9)$. (B) Grand averages of ABR waveform \pm SEM from $S p n b^{q v-3 J / q v-3 J}$ mice are broadened and delayed, while amplitudes are less affected. (C) ABR latencies increase progressively from wave I to IV between $S p n b^{q v-3 J / q v-3 J}$ and $S p n b^{+/+}$mice.

Single unit recordings were measured from the AN of $S p n b^{q v-3 J / q v-3 J}$ mice. Peak rate and steady-state rate were both reduced to approximately the same amount (see Fig. 1.9 AB). The first spike latency (FSL) showed a slight trend towards delayed spiking, but was not significantly longer or more jittered in SGNs of Spnb ${ }^{q v-3 J / q v-3 J}$ mice (see Fig. 1.9 C). Furthermore, spontaneous spike rate was slightly reduced (see Fig. 1.9 A-D). In contrast, responses to forward masking showed no depression of spiking and did not provide any indication of a deficit in recovery from adaptation (see Fig. 1.9 E). Furthermore, phaselocking was not different between SGNs of $S p n b^{q v-3 J / q v-3 J}$ mice compared to wildtype littermates, although the number of recordings was too small for meaningful statistical testing (see Fig. 1.9 F).

In cells from the AVCN, the spike rate reduction is partially compensated. In GBCs peak rate was not different between $S p n b^{q v-3 J / q v-3 J}$ and $S p n b^{+/+}$mice, but steady-state rate 

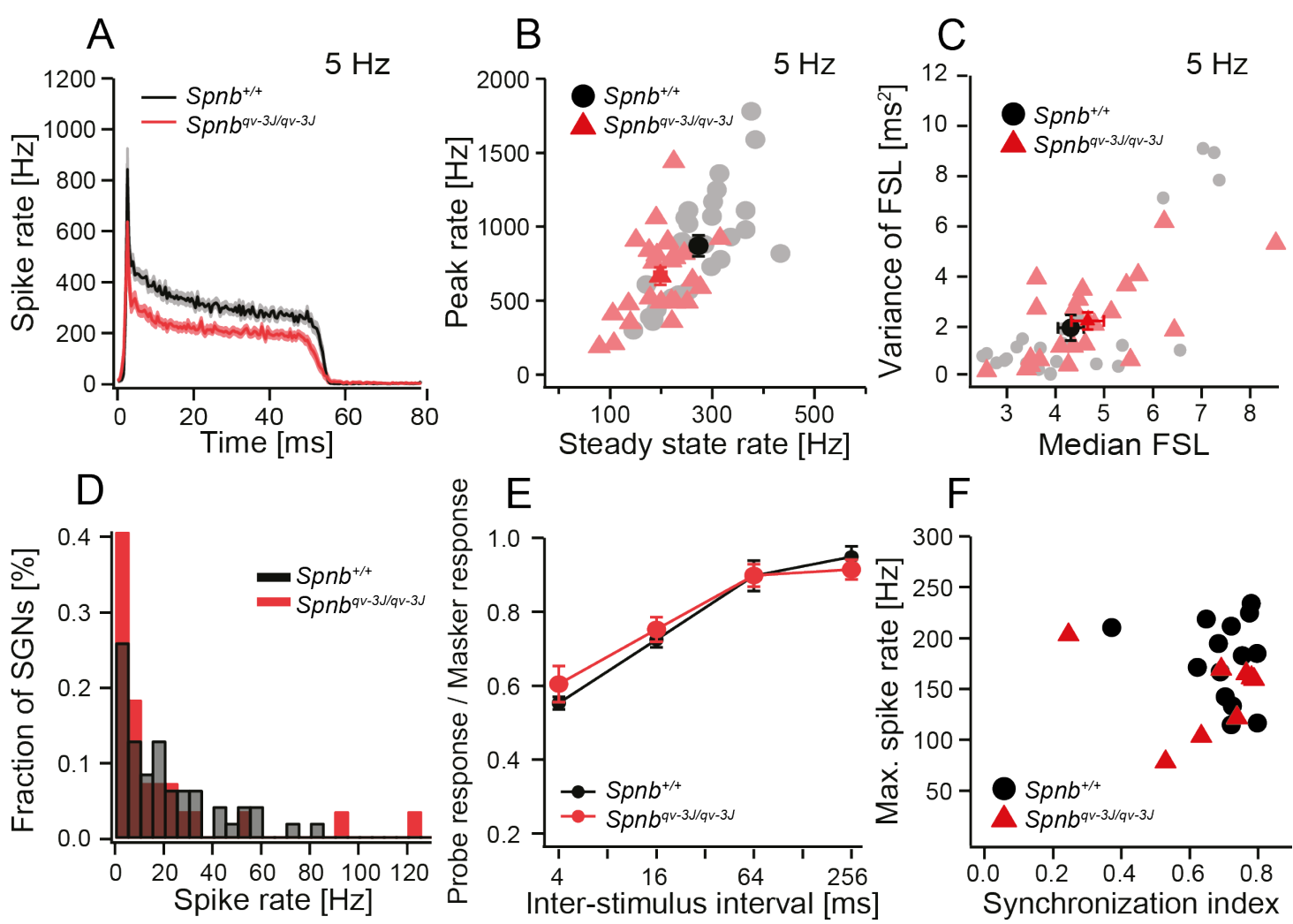

Figure 1.9.: Responses from SGNs of $S p n b^{q v-3 J / q v-3 J}$ mice. Adapted from Yamanbaeva 2017.

(A) Averaged peak-aligned PSTH \pm SEM in response to $50 \mathrm{~ms}$ tone bursts presented at $\mathrm{CF}$, $30 \mathrm{~dB}$ above threshold at a stimulus rate of $5 \mathrm{~Hz}$. (B) Peak rate, as well as steady-state rate were reduced in SGNs from $S p n b^{q v-3 J / q v-3 J}$ mice $(\mathrm{n}=25)$ compared to $S p n b^{+/+}$littermates $(\mathrm{n}=28)$. (C) First-spike latencies tended to be increased and more jittered in SGNs from Spnb ${ }^{q v-3 J / q v-3 J}$ mice, although no significant difference could be shown. (D) Spontaneous rate was reduced in SGNs from Spnb ${ }^{q v-3 J / q v-3 J}$ mice $(\mathrm{n}=21)$ compared to $S p n b^{+/+}$littermates $(\mathrm{n}=40)$. (E) Forward masking revealed a normal recovery and no increased depression of spiking ( $\mathrm{n}=10 \mathrm{each}$ ). (F) Phase-locking, represented by the synchronization index was not affected in SGNs from

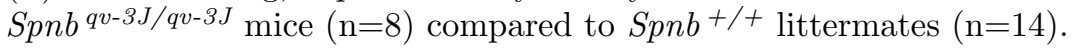

was still reduced in $S p n b^{q v-3 J / q v-3 J}$ mice (see Fig. 1.10 A). In stellate cells no differences could be found, neither for peak rate, nor for steady-state rate (see Fig. 1.10 B). The delayed ABR waveform suggested an increased FSL in neurons from the AVCN, however this could neither be supported nor rejected by statistical testing in the recordings form GBCs and stellate cells. 


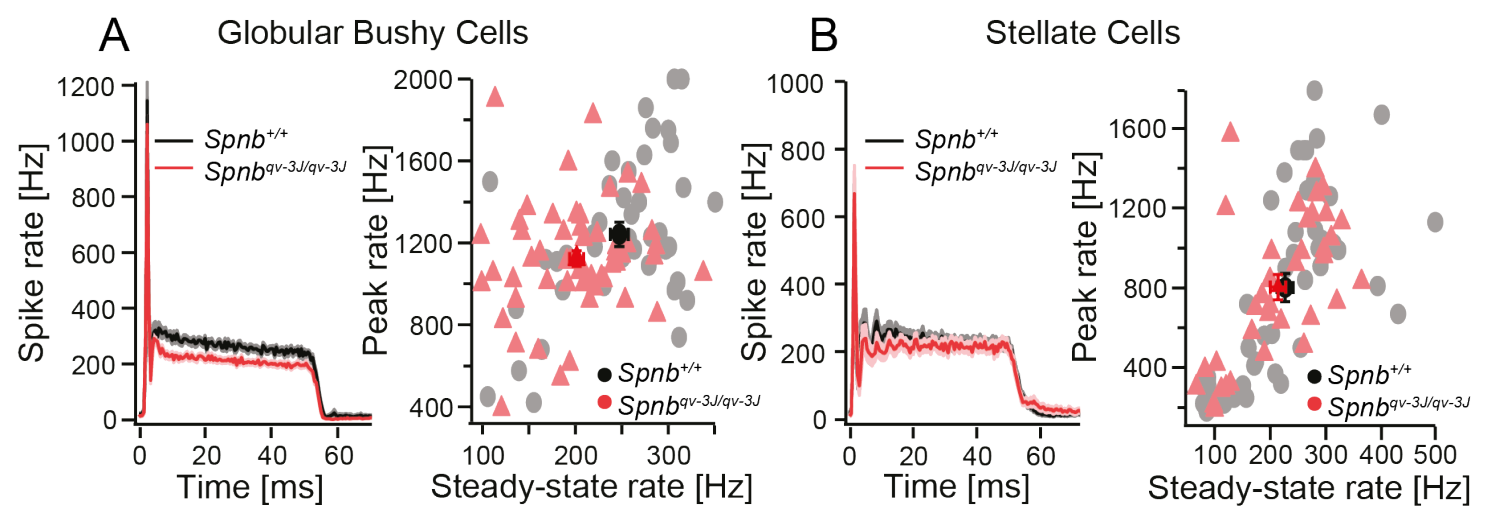

Figure 1.10.: Responses of neurons from the AVCN of $S p n b^{q v-3 J / q v-3 J}$ mice. Adapted from Yamanbaeva 2017.

(A) Averaged peak-aligned PSTH $\pm \mathrm{SEM}$ from GBCs of $S p n b^{q v-3 J / q v-3 J}$ mice in response to $50 \mathrm{~ms}$ tone bursts presented at CF, $30 \mathrm{~dB}$ above threshold at a stimulus rate of $5 \mathrm{~Hz}$. (B) Peak rate was normal, while steady-state rate was reduced in GBCs of $S p n b^{q v-3 J / q v-3 J}$ mice $(\mathrm{n}=54)$ compared to $S p n b^{+/+}$littermates $(\mathrm{n}=42)$, showing a partial compensation. (C) Averaged peakaligned PSTH \pm SEM from stellate cells of $S p n b^{q v-3 J / q v-3 J}$ mice in response to $50 \mathrm{~ms}$ tone bursts presented at $\mathrm{CF}, 30 \mathrm{~dB}$ above threshold at a stimulus rate of $5 \mathrm{~Hz}$. (E) Peak rate, as well as steady-state rate were comparable between stellate cells of $S p n b^{q v-3 J / q v-3 J}$ mice $(\mathrm{n}=36)$ compared to $S p n b^{+/+}$littermates $(\mathrm{n}=45)$, showing a full compensation in these cells. 


\section{Aim of the study}

Significant research has already been conducted on the question how sounds are encoded in the IC but nevertheless, we are still far from understanding precisely how this midbrain nucleus contributes to sound perception. While peripheral auditory coding in SGNs is understood relatively well, our knowledge regarding the connectivity and function of higher centers of the auditory pathway decreases as the tasks of the relevant nuclei become more complex. Here, we used a rarely utilized approach of studying the consequences of hearing dysfunction on IC responses in order to learn more about the function of the auditory pathway and the disease mechanisms of hearing loss. We specifically were interested in animal models of human auditory synaptopathy and neuropathy, which have genetic mutations leading to malfunction in the IHC ribbon synapse or the neurons of the auditory pathway, while having a mostly intact cochlear amplification. Some of those patients are deaf, whereas others have hearing impairments in which simple sound amplification by hearing aids is not helpful, due to a deficit in peripheral or central processing.

In this study we are investigating central sound encoding in the ICC from mouse models with auditory neuro-/synaptopathies. These mouse lines have specific, well-defined mutations in proteins that lead to hearing impairments and have already been extensively characterized by analyzing the auditory coding in single SGNs and AVCN neurons.

We recorded single neuron responses from the ICC of anesthetized mice, while playing different sound patterns to them. We compared the results of these recordings to the previously obtained data from in vivo single unit recordings from the AN and the AVCN. This way we got insights into how sounds are processed between the beginning of the auditory pathway, namely the SGNs, and the ICC in the midbrain. By comparing data from mutant mice with their wildtype littermate controls, we wanted to investigate how the auditory brainstem deals with slightly erroneous input signals.

The selected mutations reflect diseases discovered in human patients with auditory synaptopathy or neuropathy. Further analysis of these mouse lines might help generating better clinical test to diagnose auditory neuropathies or help improving treatments for these patients.

We chose different kinds of mouse lines with different phenotypes in the SGNs, to look at the picture from various sides. The different approaches consist of a mutation that is only present in the peripheral IHCs, to see how defects, which are only arising at the beginning of the auditory pathway, are propagated to the ICC. In contrast, a second mutation is present in all neurons throughout the auditory pathway to see if and how defects might accumulate up to the ICC. Another approach is to compare a mutation 
leading to only a mild hearing impairment with a similar mutation in the same protein, leading to a severe hearing impairment.

In detail, the different mutations are

$B s n^{f l / f l}$ Pres ${ }^{C r e}$ — a hair-cell specific bassoon deletion leading to impaired ribbon anchoring in the IHCs and halved spike rates in SGNs (mild auditory synaptopathy).

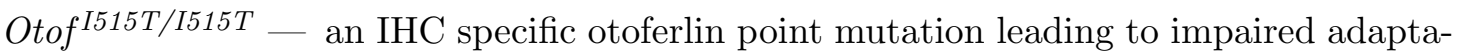
tion to continuous sounds and auditory fatigue (auditory synaptopathy).

Otof Pga/Pga - a similar otoferlin mutation that leads to deafness with almost, but not completely abolished neurotransmitter exocytosis to the synaptic cleft (severe auditory synaptopathy).

$S p n b^{q v-3 J / q v-3 J}$ - a spectrin mutation leading to a spike initiation deficit and reduced spike propagation velocity accumulating throughout the whole auditory pathway (auditory neuropathy).

With this approach we want to address the following research questions:

- How are different features of peripheral sound encoding like frequency tuning, and intensity coding, spike rates and spike timing precision, phase-locking, adaptation and recovery from forward masking processed in the brainstem and how are they coded in the ICC?

- What are the effects of peripheral sound encoding deficits in the IHC ribbon synapse on sound encoding in the ICC?

- What are the effects of an auditory neuropathy affecting all neurons in the brainstem on sound encoding in the ICC?

- To what extend is the auditory pathway able to compensate peripheral sound encoding deficits?

- What can we learn about human auditory neuropathies from the mouse models?

- Do we find specific deficits in sound encoding in animal models of human auditory synaptopathy and neuropathy which could potentially be used in the diagnosis and therapy of human hearing disorders? 


\section{Material and Methods}

\subsection{Animals}

All experiments were conducted in accordance with national animal care guidelines and were approved by the University of Göttingen Board for animal welfare and the animal welfare office of the state of Lower Saxony (approval number AZ 33.9-42502-04-15/1998). 6-12 week old mice were used for single unit recordings from the ICC. Immunohistochemical stainings were performed on 9-weeks-old mice and recordings of ABRs were performed on mice at the age of 5-6 weeks or 12-14 weeks. The number of animals used for the different experiments is shown in table 1.

Table 1.: Animal numbers

\begin{tabular}{|c|c|c|c|}
\hline Experiment & Strain & $\begin{array}{l}\text { Wildtype } \\
\text { animals }\end{array}$ & $\begin{array}{l}\text { Mutant } \\
\text { animals }\end{array}$ \\
\hline \multirow[t]{3}{*}{ IC recordings } & Otof ${ }^{I 515 T / I 515 T}$ & 13 & 14 \\
\hline & Otof Pga/Pga & & 2 \\
\hline & $S p n b^{q v-3 J / q v-3 J}$ & 14 & 16 \\
\hline Immunohistochemistry & $B s n^{f l / f l}$ Pres ${ }^{C r e}$ & 2 & 2 \\
\hline \multirow[t]{3}{*}{ ABR recordings } & $\begin{array}{l}\text { Bsn }{ }^{f l / f l} \text { Pres } C r e \\
(5-6 \text { weeks })\end{array}$ & 4 & 4 \\
\hline & $\begin{array}{l}\text { Bsn }{ }^{f l / f l} \text { Pres } \text { Cre } \\
(12-14 \text { weeks })\end{array}$ & 2 & 2 \\
\hline & $\mathrm{Bsn}^{+/+}$Pres ${ }^{C r e}$ & 2 & \\
\hline
\end{tabular}

\subsubsection{Otof ${ }^{1515 T / I 515 T}$ mice}

Otof I515T/I515T mice were kindly provided by Dr. Ellen Reisinger. These mice are homozygous for a point mutation in the Otof gene coding for otoferlin, where an isoleucine was replaced with a threonine in the $\mathrm{C} 2 \mathrm{C}$ domain. They were generated by targeted mutagenesis of a genomic vector and homologous recombination in mouse embryonic stem cells and backcrossed to CBA/J background for five generations as described before (Strenzke et al. 2016). The strain was maintained by heterozygous breeding. 


\subsubsection{Otof ${ }^{P g a / P g a}$ mice}

Otof ${ }^{P g a / P g a}$ (pachanga) mice have an aspartate to glycine substitution in the C2F domain of Otoferlin, leading to drastically reduced otoferlin levels in inner hair cells. These mice were generated and identified by a forward genetics screen using N-ethyl-N-nitrosourea (ENU) as a mutagenic substance to find recessive forms of deafness (Schwander et al. 2007). The auditory phenotype was further characterized by Pangrsic et al. 2010. The genotype of this mouse strain was maintained by homozygous breeding. All offspring of this line were homozygous for the pachanga mutation and no wildtype littermates were available.

\subsection{3. $B s n^{f l / f l}$ Pres $^{\text {Cre }}$ mice}

Hair-cell specific bassoon knock-out mice were generated by crossing a floxed bassoon mouse with a prestin-cre mouse. Floxed bassoon mice $\left(B S N^{f l / f l}\right)$, where the bassoon gene is flanked by LoxP sites in all cells, were kindly provided by Prof. Dr. Anna Fejtova (Universitätsklinikum Erlangen, former: Leibniz Institute of Neurobiology, Magdeburg). Prestin-cre mice (Pres ${ }^{\text {cre-pos }}$ ) were kindly provided by Prof. Dr. Jutta Engel (Universität des Saarlandes, Homburg) and express a cre-recombinase under the prestin promotor. Although prestin is an $\mathrm{OHC}$ specific protein, the Prestin-Cre line has been demonstrated to express the cre-recombinase also in IHCs (Tian et al. 2004)

$$
B s n^{f l / f l} \quad x \quad \text { Pres }{ }^{\text {Cre-pos }}
$$

From the offspring, which are heterozygous for LoxP sites, Cre positive mice were bread with Cre negative mice.

$$
\mathrm{Bsn}^{\mathrm{fl} /+} \text { Pres }{ }^{\text {Cre-pos }} \quad x \quad \mathrm{Bsn}^{\mathrm{fl} /+} \text { Pres }{ }^{\text {Cre-neg }}
$$

The offspring of this breeding, which were homozygous for LoxP sites were further used for continuous breeding and maintenance of this mouse line.

$$
B s n^{f l / f l} \text { Pres Cre-pos } x \quad B s n^{f l / f l} \text { Pres Cre-neg }
$$

\subsection{4. $S p n b^{q v-3 J / q v-3 J}$ mice}

The so-called "quivering" mutation describes a set of different mutations in the Spnb gene (synonym Sptbn), leading to a quivering phenotype in mice with auditory and motor neuropathies. It arose spontaneously in the department of zoology at the Ohio state university (Yoon and Les 1957) and different spontaneous variants were discovered leading to several truncated forms of $\beta \mathrm{IV}$ spectrin with different protein length. We used quivering 3 mice from the Jackson Laboratory (Sptbn $4{ }^{q v-3 J}$, JAX stock \#001028) with a C57Bl/6 background, which were first described by Rauch 1987. These Spnb ${ }^{q v-3 J / q v-3 J}$ mice carry a $\mathrm{T}$ insertion in the Spnb gene that produces a frameshift and were further characterized by Parkinson et al. 2001. 


\subsection{Immunohistochemistry}

Table 3.: Immunohistochemistry setup equipment

\begin{tabular}{ll} 
Equipment & Company \\
\hline Cryostat & \\
2800 Frigocut E & Reichert-Jung, Depew, New York \\
\hline
\end{tabular}

\begin{tabular}{ll}
\hline \multicolumn{2}{l}{ Confocal laser scanning microscope } \\
Leica TCS SP5 & Leica Microsystems, Wetzlar, Germany \\
\hline lasers & $488 \mathrm{~nm}(\mathrm{Ar})$ \\
& $561 \mathrm{~nm}$ CW-DPSS \\
& $633 \mathrm{~nm}(\mathrm{He}-\mathrm{Ne})$ \\
\hline objectives & Leica HCX PL APO CS $63 \times / 1.40-0.60$ OIL \\
& Leica HC PL APO CS $10 \times / 0.40$
\end{tabular}

Table 5.: Immunohistochemistry material

\begin{tabular}{ll} 
Consumables & Company \\
\hline Phosphate buffered saline (PBS) & Sigma-Aldrich, St. Louis, Missouri \\
\hline 37 \% FA (used as 4 \% diluted in PBS) & Merck, Darmstadt, Germany \\
\hline Mowiol 4-88 & Carl Roth, Karlsruhe, Germany \\
\hline Triton-X-100 & Merck, Darmstadt, Germany \\
\hline Tissue Tek Cryomatrix & $\begin{array}{l}\text { Thermo Fisher Scientific, Waltham, } \\
\text { Massachusetts }\end{array}$ \\
\hline Parafilm & Bemis Company, Neenah, Wisconsin \\
\hline SuperFrost Plus microscopy slides & $\begin{array}{l}\text { Thermo Fisher Scientific, Waltham, } \\
\text { Massachusetts }\end{array}$ \\
\hline NaCl & Carl Roth, Karlsruhe, Germany \\
\hline Na2HPO4 & Merck, Darmstadt, Germany \\
\hline NaH2PO4 & Merck, Darmstadt, Germany \\
\hline Goat serum & Merck, Darmstadt, Germany \\
\hline Sucrose & Merck, Darmstadt, Germany
\end{tabular}


Table 7.: Immunohistochemistry recipes

\begin{tabular}{ll} 
Buffer & Ingredients \\
\hline PB $(240 \mathrm{mM})$ & $27.52 \mathrm{~g} \mathrm{Na} 2 \mathrm{HPO} 4$ \\
(phosphate buffer) & $5.52 \mathrm{~g} \mathrm{NaH} 2 \mathrm{PO} 4$ \\
& in $1 \mathrm{~L} \mathrm{H} 2 \mathrm{O}$ \\
\hline GSDB & $16 \%$ goat serum \\
(goat serum dilution buffer) & $450 \mathrm{mM} \mathrm{NaCl}$ \\
& $0.3 \%$ Triton-X-100 \\
& $20 \mathrm{mM} \mathrm{PB}$
\end{tabular}

Table 9.: Antibodies

\begin{tabular}{llll} 
Antibodies & Company & Cat.No. & Dilution \\
\hline mouse-Sap7f & Abcam, Cambridge, United Kingdom & ab82958 & $1: 200$ \\
\hline rabbit-Ribeye-A & SYnaptic Systems, Göttingen, Germany & 192103 & $1: 500$ \\
\hline \multicolumn{5}{l}{} \\
\hline $\begin{array}{l}\text { mouse-PSD95 } \\
\text { rabbit-Ribeye-A }\end{array}$ & SYnaptic Systems, Göttingen, Germany & 192103 & $1: 500$ \\
\hline $\begin{array}{l}\text { goat-anti- } \\
\text { rabbit-488 }\end{array}$ & $\begin{array}{l}\text { Thermo Fisher } \\
\text { Massachusetts }\end{array}$ & Scientific, Waltham, A-11008 & $1: 200$ \\
\hline $\begin{array}{l}\text { goat-anti- } \\
\text { mouse-568 }\end{array}$ & $\begin{array}{l}\text { Thermo Fisher } \\
\text { Massachusetts }\end{array}$ & Scientific, Waltham, A-11004 & $1: 200$
\end{tabular}

\subsubsection{Preparation of the organ of Corti}

In preparation for immunohistochemical stainings, the organs of Corti needed to be extracted from the mice. Therefore, the animals were decapitated and both petrous bones were isolated and further moved to a petri dish filled with cold phosphate buffered saline (PBS). With the help of forceps and a preparation microscope, the auditory bulla and the ossicles were removed to expose the cochlea. A small perforation was made into the bony apex of the cochlea. The whole preparation was transferred into a petri dish with $4 \%$ formaldehyde (FA) and fixed for 10 minutes on ice while the cochlea was additionally perfused with FA via a syringe through the round window and the perforation in the apex. Afterwards the preparation was moved back into PBS and the bony parts of the cochlea were carefully removed. The stria vascularis was taken off and 
the apical turn of the organ of Corti was obtained by severing the modiolus at the middle of the cochlea.

\subsubsection{Fluorescent immunohistochemistry}

A 24-well plate was covered with parafilm and a small dent was pushed in the parafilm and filled with PBS on each well to have a tiny reaction site. Each organ of Corti was transferred to its own reaction site in the well plate. Firstly, the PBS was replaced with $100 \mu \mathrm{l}$ goat serum dilution buffer (GSDB) per well and incubated for 60 minutes at room temperature to block unspecific antibody binding sites. The primary antibodies were diluted in $100 \mu \mathrm{l}$ GSDB per well and applied over night at $4{ }^{\circ} \mathrm{C}$ in a wetbox. After three washing steps of 10 minutes in PBS the secondary antibodies were applied in $100 \mu \mathrm{l}$ GSDB per well for 2 hours at room temperature in a dark wetbox. After washing again three times for 10 minutes in PBS the organs of Corti were transferred to an objective slide and mounted with mowiol mounting medium and a cover slip.

\subsubsection{Preparation of brain cryoslices}

For some experiments, after recording of single units from the inferior colliculus, the whole brain was taken out and sliced to check whether the site of recording has been the ICC. Therefore, the recording electrode was covered in the fluorescent marker DiI and reentered for a short time period into the former recording site. Afterwards, the brain of the animal was prepared out of the skull, taking care especially not to harm the midbrain. The brains were fixed in $4 \% \mathrm{FA}$ overnight and afterwards stored in a solution of $30 \%$ sucrose for cryoprotection. Brains were mounted on a preparation holder in the cryostat with cryomatrix and sliced in $20 \mu \mathrm{m}$ sections. Once reaching the IC, every third slice was transferred to an objective slide.

\subsubsection{Confocal microscopy}

Immunohistochemical stainings were imaged with a light-scanning confocal microscope equipped with a $63 \mathrm{x}$ oil-immersion objective. Z-stacks were acquired to get a picture of the whole row of inner hair cells and all ribbon synapses.

Brain slices were imaged with the same microscope using a 10x dry objective. Single plain images were taken from all areas covering whole brain slice, making sure to have at least $10 \%$ overlap between the images. These single images were then stitched together using a stitching plugin from Fiji/ImageJ (see Fig. 3.1 B). 


\subsection{Auditory brainstem responses}

Table 11.: ABR Setup equipment

\begin{tabular}{ll} 
Equipment & Company \\
\hline Heating blanket & $\begin{array}{l}\text { Hugo Sachs Elektronik-Harvard Apparatus, } \\
\text { March-Hugstetten, Germany }\end{array}$ \\
\hline TDT System II & Tucker-Davis Technologies, Alachua, Florida \\
\hline JBL 2402 speaker & JBL, Northridge, Los Angeles \\
\hline $1 / 4^{\prime \prime}$ calibration microphone (D 4039) & Brüel\&Kjaer, Nærum, Denmark \\
\hline Neuroamp amplifier & Custom-build \\
\hline Ketamine $10 \%(125 \mathrm{mg} / \mathrm{kg})$ & $\begin{array}{l}\text { Medistar Arzneimittelvertrieb, } \\
\text { Ascheberg, Germany }\end{array}$ \\
\hline Xylazine $2 \%(2.5 \mathrm{mg} / \mathrm{kg})$ & $\begin{array}{l}\text { Riemser Pharma, Greifswald - Insel Riems, } \\
\text { Germany }\end{array}$
\end{tabular}

\subsubsection{Recording procedure}

ABR recordings were performed by Nadine Dietrich. Mice were anesthetized with an intra-peritoneal injection of ketamine $(125 \mathrm{mg} / \mathrm{kg})$ and xylazine $(2.5 \mathrm{mg} / \mathrm{kg})$ and placed in a sound shielded room. For maintaining the body temperature at $37{ }^{\circ} \mathrm{C}$, mice were placed on a rectal temperature-controlled heading pad. For monitoring the anesthesia, the heart rate was constantly tracked. Three electrodes were positioned subcutaneously underneath the pinna, on the vertex and near the tail as a common ground. Stimulus generation, presentation and data acquisition was done by a TDT II System run by BioSig software. ABRs were recorded by presentation of two repetitions of 1300 (2000 for $80 \mathrm{~dB}$ click) responses to $12 \mathrm{~ms}$ long tone bursts, equipped with $1 \mathrm{~ms} \cos ^{2}$-ramps, with frequencies of $4,6,8,12,16,24$, and $32 \mathrm{kHz}$ presented at $40 \mathrm{~Hz}$ or in response to $0.03 \mathrm{~ms}$ long clicks presented at $20 \mathrm{~Hz}$ and $100 \mathrm{~Hz}$ at sound pressure levels between $10 \mathrm{~dB}$ SPL and $100 \mathrm{~dB}$ SPL in $10 \mathrm{~dB}$ steps. Sounds were presented by a free-field loudspeaker, which was placed in front of the ipsilateral ear compared to the recording site. The ABR signal was measured by recording the potential between the vertex and mastoid subdermal needle, amplifying the signal 50000 times, filtering it (low pass: $400 \mathrm{~Hz}$, high pass: $4000 \mathrm{~Hz}$ ) and sampling it at a rate of $50 \mathrm{kHz}$ for $20 \mathrm{~ms}$. The hearing threshold was determined by visual inspection of the experimenter and a second independent observer at the lowest stimulus intensity that evoked a reproducible response. 


\subsection{In vivo single unit recordings from the inferior colliculus}

Table 13.: IC recording setup equipment

\begin{tabular}{ll} 
Equipment & Company \\
\hline Soundproof chamber & IAC, Niederkrüchten, Germany \\
\hline Heating plate & Custom-build \\
\hline Stereotaxic system & Custom-build \\
\hline LN Junior 4RE micro manipulator & Luigs \& Neumann, Ratingen, Germany \\
\hline Digital Lynx SX, cheetah data acquisition & Neuralinx, Bozeman, Montana \\
\hline NI DAQ card & National Instruments, Austin, Texas \\
\hline Loudspeaker Viva & Avisoft Bioacoustics, Glienicke, Germany \\
\hline 1/4" calibration microphone (D 4039) & Brüel\&Kjaer, Nærum, Denmark \\
\hline Measurement amplifier (2610) & Brüel\&Kjaer, Nærum, Denmark \\
\hline Head holder & Custom-build \\
\hline V200 LED Curing Light & Guilin veirun medical technology, \\
& Guangxi, China \\
\hline OmniDrill35 & World Precision Instruments, Sarasota, \\
\hline Leander Tec 3 Style Anesthetic Vaporizer & NorVap, Skipton, United Kingdom \\
\hline Contrafluran (isoflurane absorber) & ZeoSys, Berlin, Germany
\end{tabular}


Table 15.: IC recording consumables

Consumables

\begin{tabular}{ll}
\hline BD Plastipak (Syringe) & Becton Dickinson, Madrid, Spain \\
\hline Sterican (hypodermic-needle), 26G & B. Braun, Melsungen, Germany \\
\hline Paladur (2 component dental cement) & Kulzer, Hanau, Germany \\
\hline Orbi-Bond SE (UV-adhesive) & ORBIS DENTAL, Münster, Germany \\
\hline Drill bits $(005 ; 007)$ & Hager \& Meisinger, Neuss, Germany \\
\hline Single tungsten electrode $(5 \mathrm{M} \Omega)$ & Science Products, Hofheim, Germany \\
\hline Forene (Isoflurane) & $\begin{array}{l}\text { AbbVie Deutschland, Ludwigshafen, } \\
\text { Germany }\end{array}$ \\
\hline Temgesic (Buprenorphin) $(0.1 \mathrm{mg} / \mathrm{kg})$ & Indivior, North Chesterfield, Virginia \\
\hline $\mathrm{NaCl}(0.9 \%)$ & B. Braun, Melsungen, Germany
\end{tabular}

\subsubsection{Recording procedure}

\section{Surgical approach}

For single unit recordings from the ICC, mice were anesthetized with isoflurane inhalation narcosis $(2 \%$ at $0.3 \mathrm{l} / \mathrm{min}$ during surgery and not more than $1 \%$ at $0.3 \mathrm{l} / \mathrm{min}$ during recording) and placed in a soundproof chamber on a heating pad with a temperature of $38^{\circ} \mathrm{C}$ on a vibration-isolated table. Anesthesia was supplemented by a subcutaneous injection of buprenorphine $(0.1 \mathrm{mg} / \mathrm{kg})$ as an analgesic. The animals' skull was exposed and the insertion of the neck muscle over the right IC was removed. The skull was covered with an UV-curable glue, functioning as adhesion bridge, a tiny hole was drilled into the skull over the contralateral cortex and a reference electrode was inserted. The animals head was immobilized by gluing it to a custom-made head holder using dental cement and fixed to a custom-made stereotaxic system. A small craniotomy was made above the right IC and a single tungsten electrode was inserted into the ICC with the help of a micromanipulator (see Fig. 3.1 A).

\section{Measurements}

Potentials between the recording and the reference electrode were measured using the neuralynx system at a sampling rate of $32 \mathrm{kHz}$. Spikes were detected based on their waveform. Local field potentials were measure by bandpass filtering neuronal signals in a range of $0.1-300 \mathrm{~Hz}$, for detecting neuronal spikes in a range of $600-6000 \mathrm{~Hz}$. Designing of acoustic stimuli was implemented by a self-written MATLAB routine, which was compatible with a MATLAB routine written by Marcus Jeschke, responsible for stimulus generation and presentation, as well as online spike sorting using a NI DAQ (national instruments data acquisition) card. Acoustic stimuli were equipped with a $4 \mathrm{~ms}$ 
A

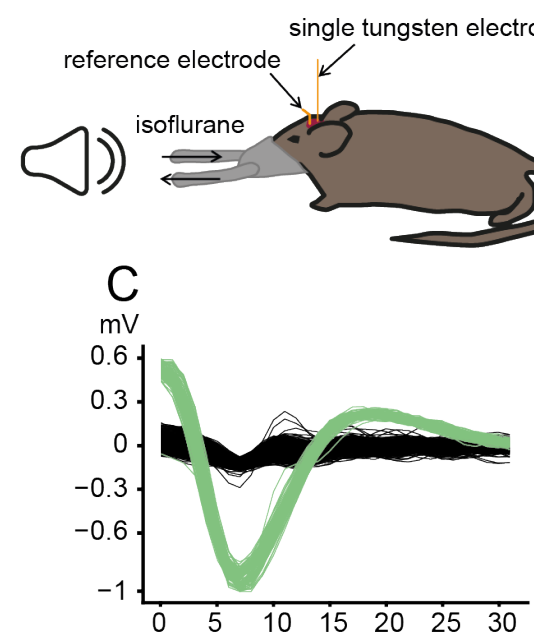

B

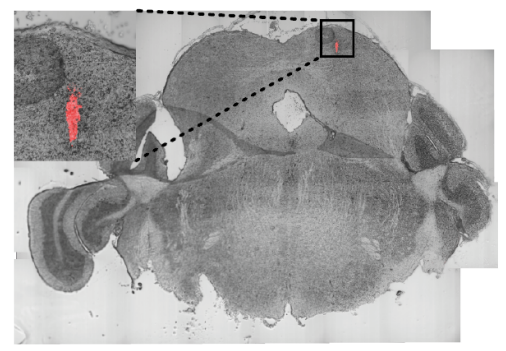

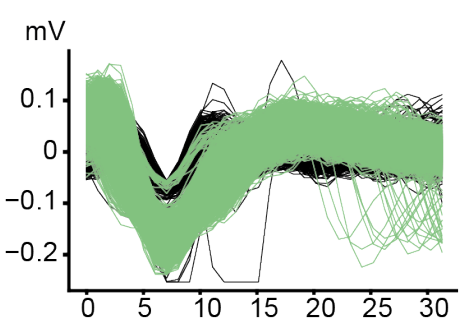

Figure 3.1.: Recording procedure

(A) Mice are anesthetized with an isoflurane inhalation narcosis during the whole recording. A reference electrode is inserted into a small hole in the skull and a small craniotomy is made into the skull over the IC for the insertion of a single tungsten electrode. (B) After the recording the electrode is coated with the fluorescent dye DiI and reinserted into the recording site. The brain of the mouse is then taken out, fixed in $4 \%$ formaldehyde and cryosectioned. The recording site in the ICC can be seen by fluorescence microscopy. (C) All recorded spikes are resorted offline after the experiment and rated according to the quality of distinction between single and multi unit spikes. Spike rating was assigned as follows. $1=$ excellent (left): all detected spikes belong to one single unit and all spikes of this unit were detected. 2 = good: almost all spikes belong to one single unit and only few spikes could be missing. 3 = fair (right): most detected spikes belong to one single unit, although a small fraction might belong to multi unit activity and a certain fraction of spikes of the single unit might not be detected. $4=$ poor: No confident separation between single and multi unit activity was possible.

$\cos ^{2}$ rise and fall ramp and presented in pseudo-random order via a calibrated free-field loudspeaker, placed $30 \mathrm{~cm}$ in front of the animals' head. Sound intensities for amplitude modulated sounds were defined as the intensity of the corresponding unmodulated sine wave.

\subsubsection{Stimulation protocol}

\section{Stimulation protocol for Otof ${ }^{I 515 T / I 515 T}$ and $S p n b^{q v-3 J / q v-3 J}$ mice}

Single units were identified by a distinguishable spiking to $100 \mathrm{~ms}$ tone bursts at $70 \mathrm{~dB}$ SPL at frequencies between $3-48 \mathrm{kHz}$ in steps of one-eighth of an octave, which were played while the electrode was very slowly moved orthogonally through the ICC. After a stable recording of a single unit was established the following stimulation protocol was run, until the protocol was finished, or the recording became unstable. 


\section{Frequency response area}

An FRA was measured by presenting $50 \mathrm{~ms}$ pure tones with frequencies between $3-$ $48 \mathrm{kHz}$ in steps of one-eighth of an octave and sound pressure levels of 40-90 dB SPL in $10 \mathrm{~dB}$ steps. Five repetitions of all tones were presented in pseudo-random order at a repetition rate of $5 \mathrm{~Hz}$. The $\mathrm{CF}$ was determined as the frequency at the lowest SPL where a response could be elicited (see also 3.5.2). Only units with an excitatory response pattern where further analyzed.

\section{Rate-level function}

Rate-level functions were measured by presenting 20 repetitions of $200 \mathrm{~ms}$ tone bursts at CF with varying levels between $0-90 \mathrm{~dB}$ SPL in $5 \mathrm{~dB}$ steps at a repetition rate of $1.5 \mathrm{~Hz}$. Threshold and optimal intensity (dBOpt), the intensity, which elicited the maximal spike rate, were selected as the first intensity that was higher than the baseline and the lowest intensity at maximal spike rate, respectively. The selection of threshold and dBOpt for monotonic fibers was eased by fitting a sigmoid function and determining the $5 \%$ and $95 \%$ quantiles (see also 3.5.3).

\section{Peri-stimulus-time-histogram}

PSTHs were recorded while playing 200 repetitions of $200 \mathrm{~ms}$ pure tones at CF and dBOpt with a stimulation frequency of $1.5 \mathrm{~Hz}$.

\section{PSTH $5 \mathrm{~Hz}$}

A second version of PSTH was recorded while playing 200 repetitions of $50 \mathrm{~ms}$ pure tones at $\mathrm{CF}$ and $\mathrm{dBOpt}$ with a stimulation frequency of $5 \mathrm{~Hz}$.

\section{Amplitude modulation}

For assessing phase-locking to amplitude modulated tones, $1000 \mathrm{~ms}$ long tones were presented with a carrier frequency at $\mathrm{CF}$ and a modulation frequency between $2-512 \mathrm{~Hz}$ in doubling steps at dBOpt with a modulation depth of $100 \% .15$ repetitions were presented at a stimulation rate of $0.4 \mathrm{~Hz}$.

\section{Forward masking}

In this paradigm a paired-tone stimulus was presented composed of a $100 \mathrm{~ms}$ masker tone at $\mathrm{CF}$ and $\mathrm{dBOpt}$ followed by a varying silent interval of 4-256 $\mathrm{ms}$ in doubling steps and a $15 \mathrm{~ms}$ probe tone at $\mathrm{CF}$ and dBOpt. 30 repetitions of the two tones were presented with an interstimulus interval of $950 \mathrm{~ms}$.

\section{Tone length}

Responses to specific tone length was assessed by presenting 20 repetitions of pure tones at $\mathrm{CF}$ and $\mathrm{dBOpt}$ with a varying duration of $8-128 \mathrm{~ms}$ in semi-doubling steps $(8,12,16$, $24,32,48,64,96,128)$ and an interstimulus interval of $283 \mathrm{~ms}$.

\section{Modulation intensity}

Neurons that responded to amplitude modulated sounds with more than some onset spikes were included in an additional analysis on coding of sAM tones. For assessing the intensity specificity, 15 repetitions of $1000 \mathrm{~ms}$ long AM tones with a carrier frequency at $\mathrm{CF}$ and a modulation frequency of $16 \mathrm{~Hz}$ were played at SPLs between 30-90 dB SPL in $10 \mathrm{~dB}$ steps at a stimulation rate of $0.4 \mathrm{~Hz}$. 


\section{Modulation depth}

The influence of modulation depth was measured by presenting 15 repetitions of $1000 \mathrm{~ms}$ long $\mathrm{AM}$ tones with a carrier frequency at $\mathrm{CF}$ and a modulation frequency of $16 \mathrm{~Hz}$ at dBOpt with varying modulation depths between $0-100 \%$ in $25 \%$ steps at a stimulation rate of $0.4 \mathrm{~Hz}$.

\section{Adaptation of the protocol for Otof ${ }^{P g a / P g a}$ mice}

As recording of single units from SGNs of Otof ${ }^{P g a / P g a}$ mice was difficult and only possible with slow stimulation and long interstimulus intervals (see chapter 1.5.4), the stimulation protocol for IC recordings from Otof ${ }^{P g a / P g a}$ mice was adjusted as follows.

In contrast to Otof $f^{I 515 T / I 515 T}$ and Spnb ${ }^{q v-3 J / q v-3 J}$ mice, single units of Otof ${ }^{\text {Pga/Pga }}$ mice were identified while playing $100 \mathrm{~ms}$ tone bursts at $90 \mathrm{~dB}$ SPL at frequencies between $3-48 \mathrm{kHz}$ in steps of one-eighth of an octave with an interstimulus interval of at least $1 \mathrm{~s}$.

FRAs were measured similar to Otof ${ }^{I 515 T / I 515 T}$ and Spnb ${ }^{q v-3 J / q v-3 J}$ mice.

Rate-level functions were measured with $100 \mathrm{~ms}$ tone bursts instead of $200 \mathrm{~ms}$ and a repetition rate of $1 \mathrm{~Hz}$.

PSTHs were measured by presenting $100 \mathrm{~ms}$ tone bursts at CF and dBOpt (90 dB for all units). Different stimulation velocities were tested with a repetition rate of $1 \mathrm{~Hz}, 0.5 \mathrm{~Hz}$ and $0.25 \mathrm{~Hz}$ with 50-200 repetitions.

Further stimulation protocols were not feasible for Otof ${ }^{P g a / P g a}$ mice, because of their inability to respond to stimulation with even moderate or high stimulation rates.

\section{Offline data processing}

Every recorded set of spikes was resorted offline with the help of another MATLAB routine written by Marcus Jeschke, using the waveform of spikes as sorting criterion and clustering them by a principal component analysis and by manual selection to ensure optimal separation between single and multi units. The quality of single unit separation was rated subjectively by a score between $1-4(1=$ excellent, $2=$ good, $3=$ fair, $4=$ poor; see Fig. $3.1 \mathrm{C}$ ), where a spike rate of 1 means every single spike of an individual neuron was detected and a spike rate of 4 means a good distinction between single and multi unit activity was not possible (see Fig. $3.1 \mathrm{C}$ ). Every measured neuron was individually examined and categorized in different classes according to its response pattern. Classification of neurons is further described in chapter 3.7. Each measured run was entered into a relational database including information about $\mathrm{CF}$, threshold, dBOpt, spike rating, unit type and detection threshold for specific runs (forward masking and tone length). Only neurons with a spike rating of 1-3 were analyzed in this study. 


\section{Calculation of spontaneous rates}

Afterwards, the spontaneous rate of a unit was calculated by taking together all runs corresponding to one single unit, excluding the very first and very last one to avoid unwanted interference from an emerging or potentially dying unit. The spike rate of the first $50 \mathrm{~ms}$ before stimulus onset was averaged over all repetitions from all runs to calculate the spontaneous firing rate of the single unit, which was then again automatically updated to the entry in the database.

\subsection{Data analysis}

\subsubsection{Database and software design}

Table 17.: Software

\begin{tabular}{lll} 
Software & Version & Company /Organization/Reference \\
\hline MATLAB & $2016 \mathrm{a}$ & The MathWorks, Natick, Massachusetts \\
\hline BioSig32 & & Tucker-Davis Technologies, Alachua, Florida \\
\hline Office Access & 2013 & Microsoft Corporation, Redmond, Washington \\
\hline Adobe Illustrator & CS5.1 & Adobe Systems Inc, San José, California \\
\hline Fiji / ImageJ2 & $1.52 \mathrm{c}$ & Schindelin et al. 2012 Rueden et al. 2017 \\
\hline \multicolumn{1}{c}{ Stitching } & 1.2 & Preibisch et al. 2009, ImageJ plugin \\
\hline \multicolumn{1}{c}{ Bio-formats } & 5.8 .2 & Linkert et al. 2010, ImageJ plugin \\
\hline $\begin{array}{l}\text { Anaconda3: Python } \\
\text { distribution }\end{array}$ & 5.0 .1 & Anaconda, Austin, Texas \\
\hline
\end{tabular}

Python $\quad 3.6 .3 \quad$ Python Software Foundation, Delaware

Table 19.: Python packages

\begin{tabular}{ll} 
Package & Version \\
\hline PyQt & 5.6 .0 \\
\hline matplotlib & 2.1 .0 \\
\hline pypyodbc & 1.3 .3 \\
\hline numpy & 1.13 .3 \\
\hline scipy & 0.19 .1
\end{tabular}


Table 21.: Software scripts provided by Marcus Jeschke

\begin{tabular}{ll} 
Script & Purpose \\
\hline ExpControl_03 & $\begin{array}{l}\text { experiment control } \\
\text { stimulus presentation } \\
\text { online data analysis } \\
\text { online spike sorting }\end{array}$ \\
\hline OnlineEval_06 & offline spike sorting \\
\hline ParseMFile & adapted from Marcus Jeschke to work in python 3
\end{tabular}

For organizing the data obtained from single unit recordings from the ICC a relational database for storing all information was created. The database was meant to be queried and give output on whatever combination of parameters could be interesting to analyze. Therefore, information about the experimenter, the animals, the experiment, the units and the single runs were included in the database. The main idea was to store the path to each individual file, that has been saved for every recorded run in the database together with the information of what was recorded. The database can be queried for all relevant information, for example everything that contains responses to amplitude modulated sounds for all animals which have a mutant genotype. The database is then able to return a list of all the files where this condition is true, which can be read one by one by a self-written analysis software. The single components of the software will be presented hereafter.

\section{Database}

The database itself was created with Microsoft Access, following and improving an existing template used for storing recordings from SGNs. It includes the tables "Users", "Animals", "Experiments", "Units" and "Runs", which are linked by 1:n cardinalities in descending order. This means that one user can be responsible for several animals, but one animal may only have one responsible experimenter. As another example, several runs can be recorded from one unit, but one run cannot origin from several units. Based on unified modeling language (UML) a scheme of the generated database, including all tables and attributes is shown in Fig. 3.2. 


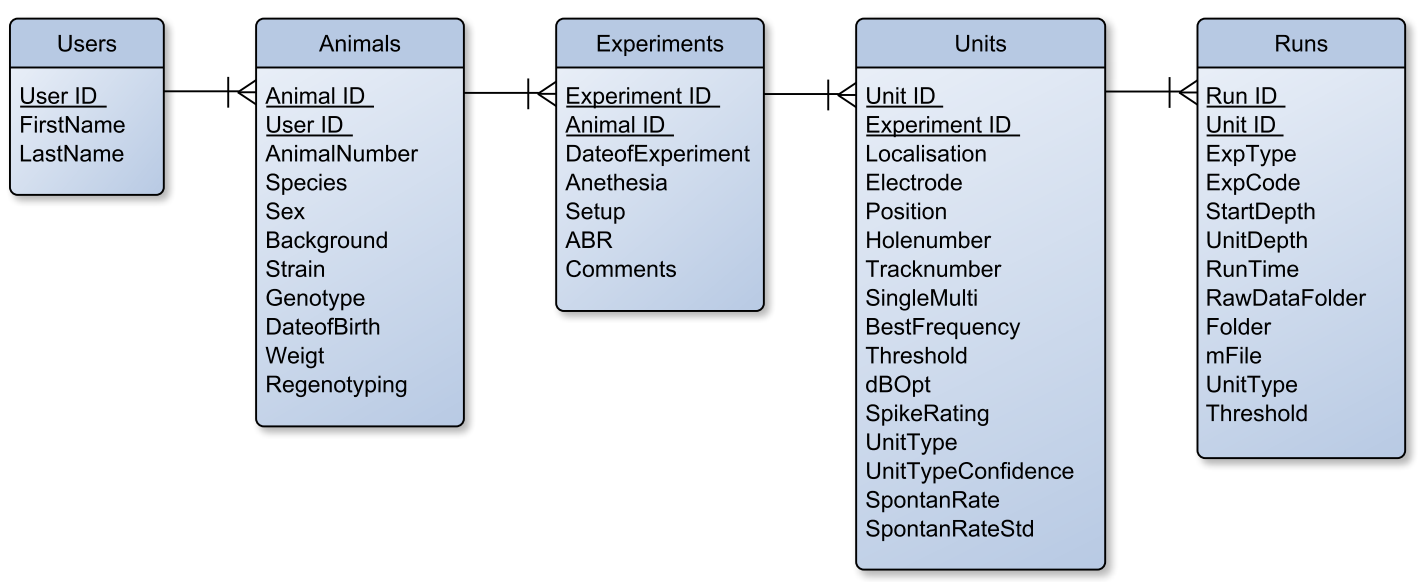

Figure 3.2.: Scheme of the generated database

The database consists of the tables Users, Animals, Experiments, Units and Runs, together containing all information about the IC recording experiments. The attributes of each table are displayed in each box, while the underscored attributes represent primary and foreign keys. The tables are linked by 1:n cardinalities in descending order.

\section{Inserting data into the database}

For inserting or updating data in the database a software was written using the python programming language. The connection to the database was established by the pypyodbc package and the main graphical user interface (GUI) was designed with the PyQt library. The software is able to read all files from one experiment and display them sorted by their run-type for each recorded unit. All files from one unit can now be displayed with the response pattern plotted as a scatter plot and optionally the waveform and some basic analysis as additional plots. Based on this information a table will be created for each run of each unit, filled automatically with all the information that will be inserted into the database and that might already exist in the database, highlighting the fields that have to be filled manually (for instance spike rating, CF, threshold) and automatically generating unique primary keys.

\section{Analyzing and plotting information from the database}

A second self-written software queries the database via an SQL (structured query language) command and returns a list of all desired files, for example all runs from only $S p n b^{q v-3 J / q v-3 J}$ mice in response to amplitude modulated tones, which have a spike rating of 3 or better. This list is then forwarded to a selected analysis routine, which will calculate and plot the selected analysis. In principle, analysis scripts can be divided into scripts that will analyze single experiment runs and plot the response of a single unit and scripts that will analyze and average a list of all given units. Some of the implemented analysis routines, which need further explanation are presented in the following chapters. 


\subsubsection{Frequency response analysis}

For an FRA, the measured frequencies and intensities were plotted on the two axis of a coordinate system, respectively. For each frequency-intensity combination the spike rate was plotted at the corresponding coordinate by a color code where dark blue represents zero spike rate and yellow represents the maximal spike rate of the unit. For more information about the firing pattern of a unit, four different variants of this plot were implemented. In addition to the absolute spike rate, each frequency-intensity combination was averaged with the neighboring frequencies and intensities to obtain a smoothed FRAplot. Further information was provided by introducing a significance criterion where the spike rate will only be plotted if for a certain condition a response was detected in at least 3 out of 5 repetitions. In addition to that, a fourth plot did not only analyze the spike rate during the presentation of the stimulus tone, but also took the spike rate before and after the stimulation into account. This allows for determining the $\mathrm{CF}$ of units, which will only, or still fire at the stimulus offset. The CF can be selected manually and will be returned by the script.

\subsubsection{Level response analysis}

\section{Rate-level functions}

For analyzing rate-level functions the averaged spike rate from all repetitions of one run was plotted over the sound intensity. The curve_fit function from the scipy.optimize python package was used to fit a sigmoid function to the data with the following formula:

$$
f(x)=\frac{G}{1+e^{-k * G *(x-x 0)}}+y 0
$$

Suggestions were provided for values of $\mathrm{x} 0, \mathrm{k}, \mathrm{G}$ and $\mathrm{y} 0$ and the curve_fit function would try to find the optimal parameters for these variables to match the data points. If no parameters could be found, for example because the single unit responded in a nonmonotonic, non-sigmoid way, a warning will be displayed.

If a sigmoid function could be fitted to the data, the dynamic range was determined by calculating the intensity range of the $10 \%$ and the $90 \%$ quantiles. For response curves where the fit failed, threshold and dBOpt were determined manually by visual inspection while dynamic range was calculated by subtracting threshold from dBOpt (see also Fig. 3.3).

For facilitating this detection and provide further information about the response patterns of the single units, a monotonicity index (MI) was calculated by dividing the spike rate at maximal intensity by the maximal spike rate. Units with a MI higher than 0.75 were defined as monotonic units and below 0.75 as non-monotonic units. 
A

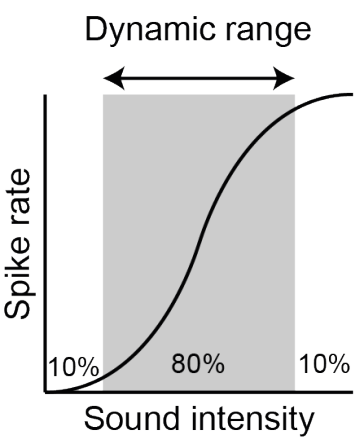

B

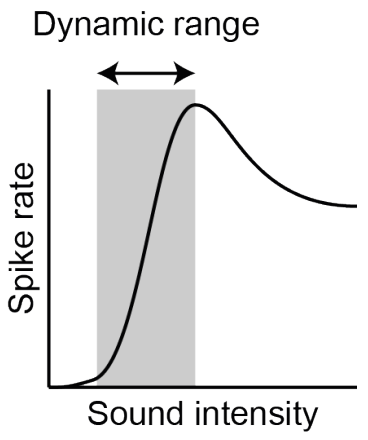

Figure 3.3.: Dynamic range calculation for monotonic and non-monotonic units. Schematics of rate-level functions from (A) monotonic single units and (B) non-monotonic single units. Dynamic range for monotonic units is calculated by fitting a sigmoid function and obtaining the $80 \%$ quantiles. Dynamic range of monotonic units is calculated by subtracting the threshold from the optimal intensity.

\section{Rate-level plots}

For analyzing and comparing rate-level functions of all measured units between genotypes the MI, threshold and dBOpt were calculated for each file as described before or taken directly from the database. For each curve the SPL was shifted relative towards the threshold and plotted on top of each other. Furthermore, the mean and maximal spike rates as well as the dynamic range were calculated for each single unit and combined in a box plot comparing all values of all single units between genotypes.

\subsubsection{Peri-stimulus-time histograms}

A PSTH shows the response of a neuron over time, averaged over many repetitions of the same stimulus. The spike rate is counted in bins with a binwidth of $2 \mathrm{~ms}$ and divided by the number of repetitions and the binwidth to get a so-called instantaneous spike rate, which can be plotted in a histogram.

In addition to that, peak-aligned PSTH were generated by adjusting the time after stimulus onset individually for each unit relative to the onset spike time, which was shifted to zero. This way the absolute onset spike rates can be compared, excluding a reduction in onset rate that can arise due to averaging several neurons with a more jittered onset spike time.

For comparing spike rates of one mouse line, all PSTHs from one genotype were taken together, averaged and plotted against the average PSTH from the other genotype.

\section{Time course of adaptation}

The time-course of adaptation was determined for each single PSTH or averaged PSTH by fitting a single exponential or double exponential function to the PSTH with the following formulas:

$$
\begin{gathered}
\text { single }: f(x)=y * e^{-x * \text { fast }}+d \\
\text { double }: f(x)=y 1 * e^{-x * \text { fast }}+y 2 * e^{-x * \text { slow }}
\end{gathered}
$$

The curve_fit function from the scipy.optimize package was used to find the optimal parameters for the different variables that fit the data points best. 


\subsubsection{First spike latency}

For calculating the FSL it was iterated through all repetitions of a single PSTH stimulus run and the time of the first spike was obtained for each repetition. The median of all first spikes from all repetitions was calculated for all single units. All medians from one genotype were taken together to calculate the median FSL from all repetitions of all fibers for each genotype.

\subsubsection{Amplitude modulation responses}

For analyzing amplitude modulated responses phase and vector strength were calculated for each unit, as well as spike rate, adaptation and failure rates. The phase and vector strength were calculated by the "vectorstrength" function provided by the scipy.signal package, which is based on the calculation after Goldberg and Brown (Goldberg and Brown 1969; van Hemmen 2013). Hereby, phase corresponds to the time point within the envelope cycle when most spikes occur, normalized to the phase duration, while the vector strength is a measure how precise the spikes occur always at the same time in the phases. A vector strength of 1 means all spikes occur always at the same phase and a vector strength of 0 means all spikes occur randomly. The rate modulation transfer function plots the average spike rate for every modulation frequency over the corresponding modulation frequency, independent of the phase-locking or vector strength. The spike decay ratio was calculated by dividing the average spike rate of the last $250 \mathrm{~ms}$ out of the $1000 \mathrm{~ms}$ stimulation by the average spike rate of the first $250 \mathrm{~ms}$. This way, the adaptation or depression of spiking over time can be analyzed. Furthermore the failure rate was calculated as the fraction of amplitude modulation cycles where no spike was elicited. Each parameter was averaged over all measured units for each modulation frequency and plotted against the corresponding modulation frequency.

\section{Phase diagram}

The phase diagram displays the spike rate in relation to the phase of an amplitude modulated tone of one single unit. Therefore, the phase length was determined for each modulation frequency and a histogram was generated over each repetition of a phase during one stimulation and over the different repetitions of the same modulation frequency. A sharp peak in the phase diagram resembles a high vector strength, while a low vector strength would result in a broader distribution of spikes in the phase diagram

\section{Circle diagram}

In the circle diagram every unit is represented by a position in a circle with the phase being represented at the circumference and the vector strength being represented at the radius. The farther outside a single point is, the better is the phase-locking while the position on the circumference represents the relative time during a phase when most spikes occurred. From all units of one genotype a resulting vector is created where the length of the vector illustrates the average vector strength and the orientation of the vector illustrates the average phase. 


\subsubsection{Forward masking analysis}

For analyzing the forward masking paradigm the spike rate for masker and for probe tone was detected for every single run. Therefore, the onset peaks for the masker and the probe response were detected by generating a window of $50 \mathrm{~ms}$, starting from the stimulus onset for maker and probe, respectively. Subsequently, the spike rate over these $50 \mathrm{~ms}$ was averaged and the spontaneous rate, taken from the database, was subtracted. Although the probe tone is only $15 \mathrm{~ms}$ long we wanted to ensure inclusion of all spikes in response to the probe tone, independent of the spike latency. The depression of spiking was calculated by dividing the spike rate of the probe response by the spike rate of the masker response.

In addition to that, an automated script was determining the minimal silent interval needed to elicit a probe response. Therefore, the script iterated through all measured responses, starting with the longest gap length and detecting the minimal silent interval if one of the following conditions was true:

- The max spike rate of the probe response minus the spontaneous rate was zero.

- Less than 3 spikes were detected in the probe response of all repetitions.

- Less than $10 \%$ of probe spikes were detected compared to the probe response at the longest gap length (256 ms)

The minimal silent interval was defined as not detected, if no probe response was elicited at any gap length or if the sum of spikes responding to the probe tone for all gap lengths was smaller than 13. For confirming this automated detection, the minimal silent interval was analyzed for all forward masking runs and inserted manually into the database, after subjective observation of a visible peak in response to the probe tone in the PSTH.

\subsection{Statistical analysis}

All statistical tests were conducted using the statistical functions of the python package scipy.stats, which were integrated into the analysis and plotting routines to receive a complete picture when analyzing data. Differences in the distribution of spontaneous rates were tested by a Kolmogorov-Smirnov test. All other samples, which should be compared, were firstly tested for normal distribution by a Shapiro-Wilks-test and for homogeneity of variances by a Levene-test. Normally distributed samples with equal variances were tested using Student's t-test. Not normally distributed samples with equal variances were tested using a Mann-Whitney-U-test. Normally distributed samples with unequal variances were tested using Welch's t-test and not normally distributed samples with unequal variances were tested using a Kruskal-Wallis-test. Significant differences are stated in the figure captions with the corresponding test and p-value. The whole statistical analysis can be seen in appendix B. 


\subsection{Characterization of unit types in the inferior colliculus}

The diversity of single unit responses within the ICC makes analyzing the recorded data difficult. Different units may have completely different spike rates, spike timings and as a matter of fact also completely different tasks. Averaging the response of all units might lead to strange results under the wrong assumptions. For instance, having a higher fraction of sustained-responding units by chance for one genotype would lead to the wrong assumption that the averaged spike rate would be higher in that group. Furthermore, the overall number of recordings is limited by the difficult experiments with a low yield of recordings per animal, on average three neurons, where the whole stimulus protocol could be applied. Significant differences are hard to show as the diversity within IC might be higher than between genotypes.

Taking together previous findings from other studies in the literature and own observations, we tried to identify recurring response patterns to different sound stimuli. This way we tried to cluster similar response patterns into different classes. However, it was not feasible to define different groups of neurons, which responded similarly. The diversity in the IC is too high and every neuron is responding individually different. For instance two neurons which showed the same response pattern for a specific task may responded completely different when challenging them with another sound pattern. We decided to group the neurons according to each presented stimulus protocol separately to get an overview on which responses occur in the ICC to which sound stimulation. Subgrouping neurons by the response patterns will reduce the risk of having false positive results but are also at risk of biasing the results by the grouping itself or by reducing the n-number for each group too much. Therefore the assignment for each unit into response classes and the selection which classes to analyze for which research question was taken with great care.

For each stimulus protocol described in chapter 3.4.2 all units from all recorded mouse lines were examined and several recurring responses could be identified for each stimulus protocol.

\subsubsection{Frequency response area}

The classification of different FRA shapes used in this thesis was similar to the one proposed by Ramachandran et al. 1999. The most abundant FRA shape was "V-shaped" neurons, which have a broad frequency tuning at high sound intensities which gets sharper at low intensities (see Fig. 3.4 A). The other shapes were "I-shaped" neurons, which have a very narrow frequency tuning across all sound levels (see Fig. 3.4 B) and "Oshaped" neurons, responding only at a specific frequency and a specific sound intensity (see Fig. 3.4 C). Shapes that were different from these three classes, like inhibitory units, which were not measured here (see Fig. 3.4 D) or the previously reported two-peak FRA (Egorova et al. 2006) got the category assignment "other". 


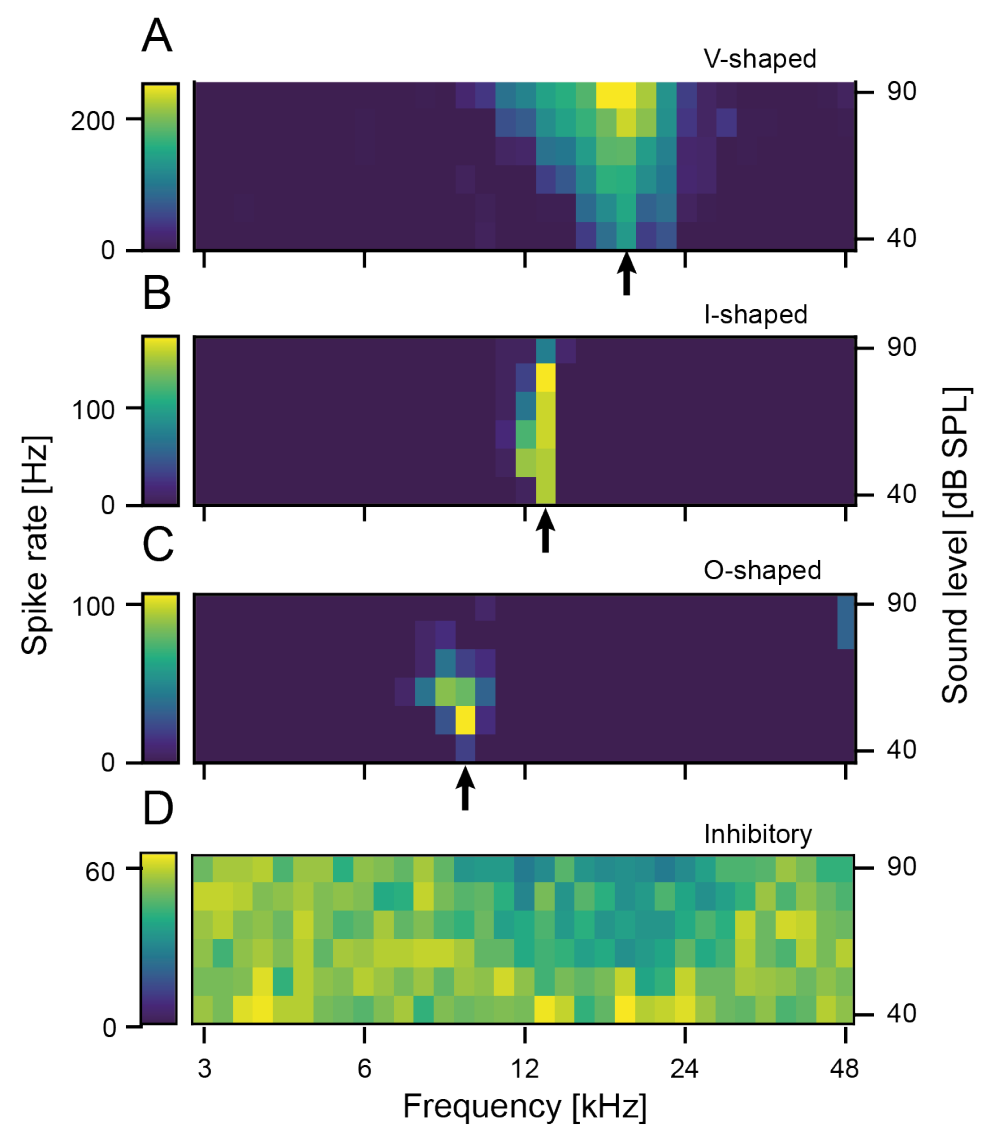

Figure 3.4.: Classes of FRAs

In the ICC, neurons with tree distinct types of FRAs were encountered. (A) Example of a "V-shaped" unit with a broad tuning at high SPLs and a sharp tuning at low SPLs. (B) Example of an "I-shaped" unit with a sharp tuning across a range of intensities. (C) Example of an "O-shaped" unit with a precise tuning to a specific frequency and intensity. (D) Example of an inhibitory neuron with a high spontaneous rate and a depression of spiking at certain frequencies. Inhibitory neurons were encountered occasionally but were not recorded from and excluded from all analysis. Arrows mark CFs.

\subsubsection{Rate-level function}

When looking at rate-level functions from SGNs, they all have a similar sigmoidal shape, such as the spike rate rises after crossing a threshold monotonically with sound intensity until it reaches a plateau. When recording multi units from the ICC all rate-level functions have a sigmoid shape. However, when examining the responses of single units, more diverse shapes can be distinguished. Only about $1 / 3$ of all recorded units obtained a sigmoid rate-level function (see Fig. 3.5 A). The second class are "peak"-shaped rate level functions, where the spike rate rises with sound intensity up to a specific best intensity and goes down again at higher sound intensities (see Fig. 3.5 C). The third class are "linear"-shaped rate level functions, where the spike rate rises linearly with sound intensity without reaching a plateau within the measured sound levels (see Fig. 3.5 C). Other, more complex shapes of rate-level functions were also encountered.

\subsubsection{Tone length}

Responses to presentations of pure tones with different tone length were divided into those which had a short response to sound onset which was independent of the actual tone length (see Fig. 3.6 A) and those neurons that would show a sustained response over the entire tone duration (see Fig. 3.6 B). Both groups were analyzed combined as well 

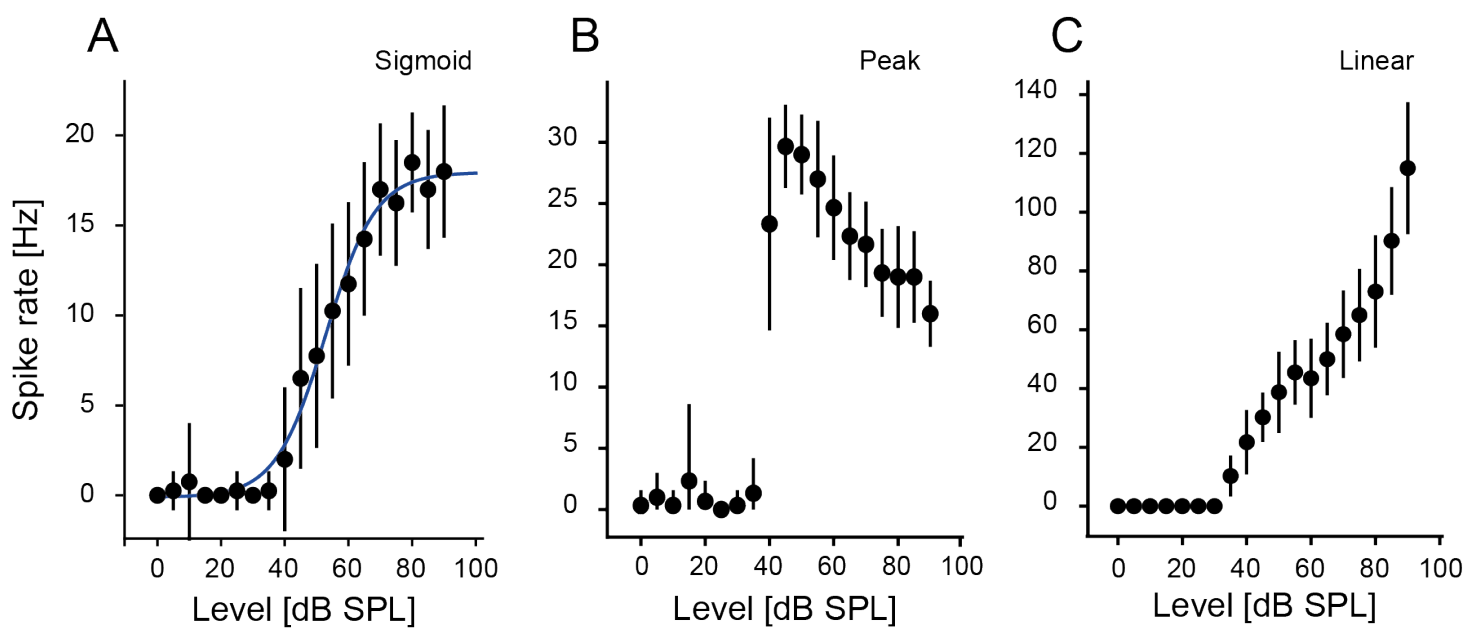

Figure 3.5.: Classes of rate-level functions.

In the ICC, neurons with tree distinct types of rate-level functions were encountered. (A) "Sigmoid" rate-level function with a rise of spike rate according to the sound intensity, reaching a plateau at a certain level. (B) "Peak" rate-level function with a maximum spike rate at a specific sound intensity and a drop of spike rate at higher SPLs. (C) "Linear" rate-level function with a linear increase in spike rate according to the sound intensity, which does not reach a plateau under the measured conditions. Data are representative single units, averaged over the measured repetitions. Error bars represent standard deviation.

as separately. Although their existence has been reported before, units with band-pass characteristics, responding specifically to one or few tone lengths were not encountered in this study (Brand et al. 2000).

Figure 3.6.: Classes of tone length responses Two kinds of responses to different tone lengths were encountered in the ICC. (A) "Onset" response units spiked only at the beginning of the tone, independent of the tone length, although not all were able to respond to very short tones. "Sustained" response units spiked throughout the length of the tones and sometimes failed for short tones as well. Grey bars represent the time when the stimulus was presented.
A

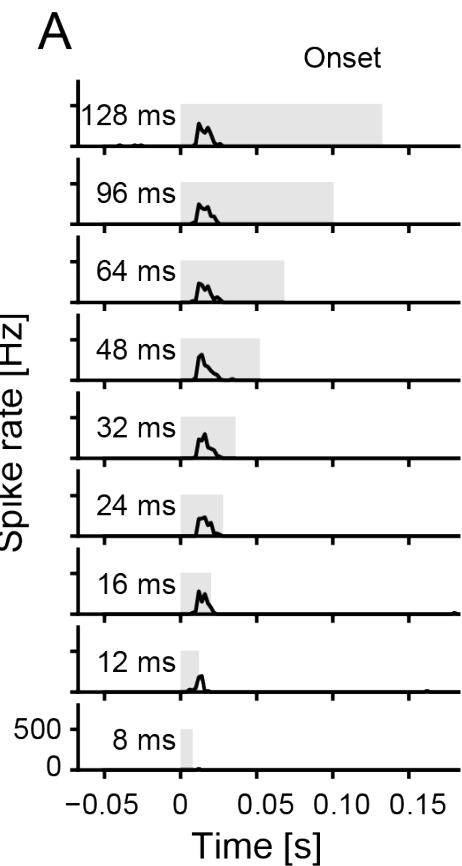




\subsubsection{Peri-stimulus time histogram}

Taking a closer look at responses to pure tone stimulation we compared PSTH shapes from ICNs. Interestingly, we were regularly able to identify similar responses as in AN and AVCN recordings, although it was not possible to connect these responses to specific cell types like in the AN and AVCN. The different classes of PSTHs consist of "primarylike" neurons, which have a sharp and strong onset response, followed by a drop of spike rate and a steady-state response (see Fig. 3.7 A). The second class contains "pri-notch" neurons, whose PSTH looked similar to those of GBCs with a prominent "notch" after the onset response (see Fig. 3.7 B). Moreover, we encountered "tonic" neurons which did not have any distinguishable onset response at all but spiked throughout the whole presentation of a tone (see Fig. 3.7 C). The fourth class is that of "onset"-neurons, which only respond very sharply at the onset of a presented sound (see Fig. 3.7D). Units of the last class, which were encountered very rarely, showed an "offset" response, spiking only after the presentation of a tone (see Fig. 3.7 E). Units that did not show any of these response properties were assigned to the category "other". The main reason to assign units to this category was due to them changing their response pattern during the stimulation. For instance by presenting the same tone in a repetitive way repeatedly, as randomization is not possible while playing the same tone, some units stop spiking after some repetitions. This phenomenon of stimulus specific adaptation has been reported before (Malmierca et al. 2009).

For analysis of tone burst runs the categories "other" and "offset" were always excluded. For the calculation of the steady-state rate the "onset" neurons which do not possess a steady-state rate were excluded as well.

\subsubsection{Amplitude modulation}

In response to sAM tones, three different classes of neurons were identified. The first class of neurons with "low-pass" characteristics were able to phase-lock to the envelope of sAM tones at low-frequency amplitude modulation up to a certain modulation frequency which they cannot keep up anymore (see Fig. 3.8 A). The second class of neurons with "band-pass" characteristics were responding specifically to one or few modulation frequencies and responded with barely more than an onset response to other modulation frequencies (see Fig. 3.8 B). The third category contains "onset" neurons, which only spike at the onset of the whole sAM tone at all tested modulation frequencies (example not shown). Units with "high-pass" characteristics were not encountered in our dataset. "Onset"-units were excluded from all analysis, as they are not responding to the envelope of sAM tones at all. "Band-pass" units needed to be excluded as well, as taking them in would bias the results when averaging all units. Phase-locking would seem impaired for one genotype, because a higher fraction of "band-pass" units to a certain modulation frequency was recorded for the other genotype, instead of an actual phase-locking deficit.

The responses to sAM tones with a modulation frequency of $16 \mathrm{~Hz}$, with varying sound intensities and modulation depths were classified similarly into the same categories (see also 

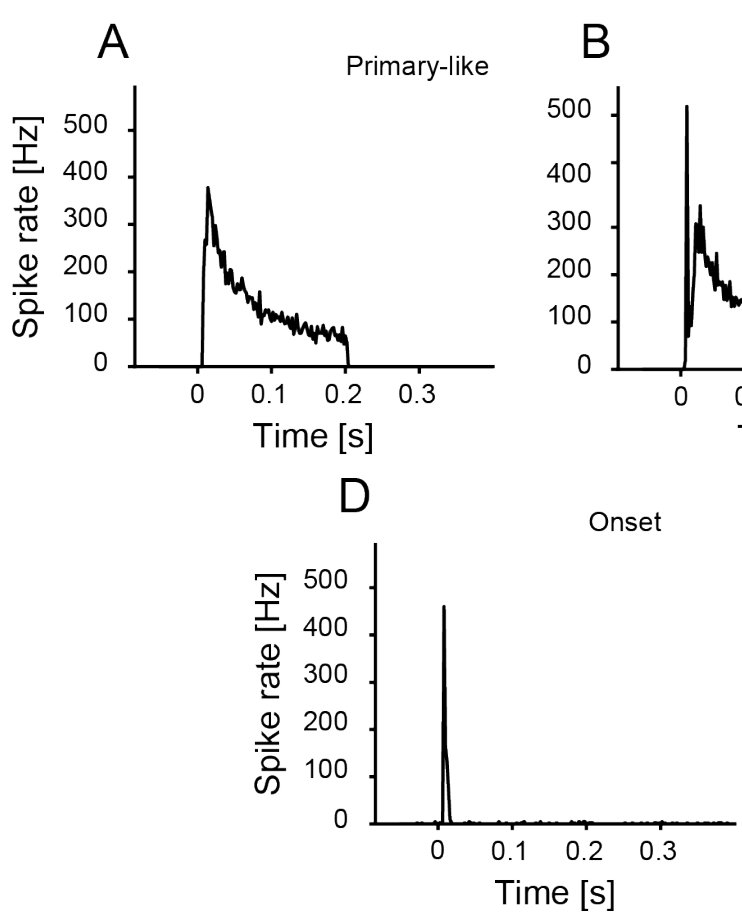
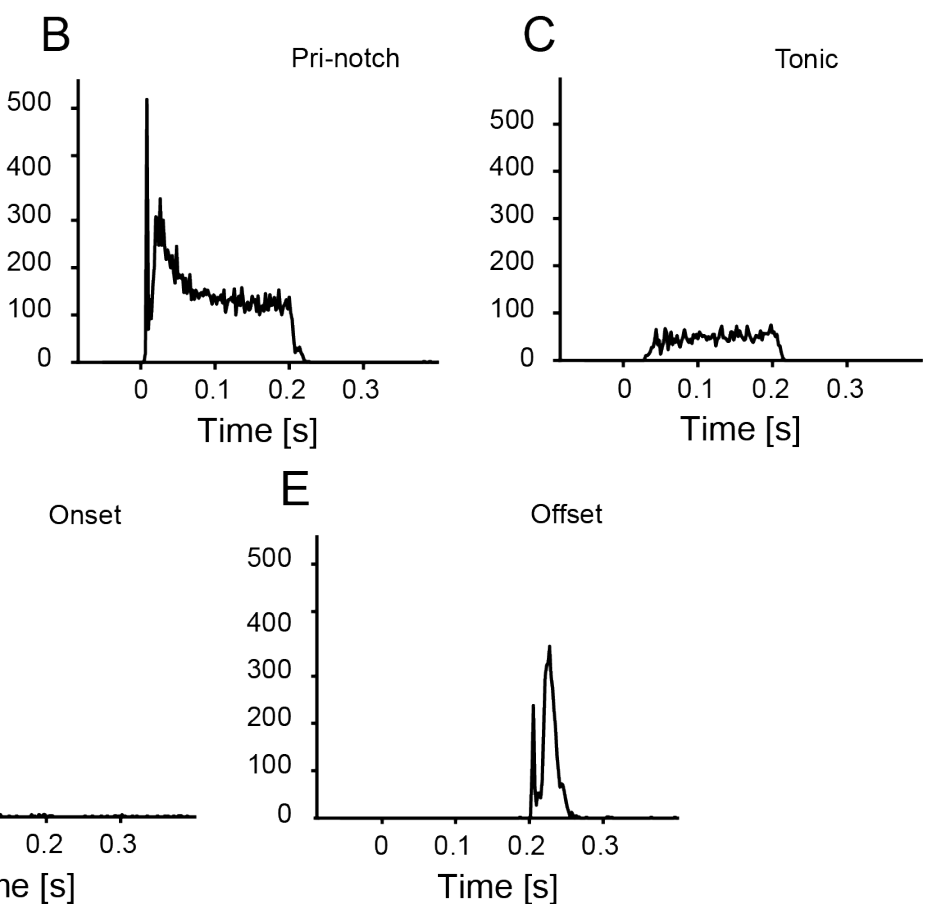

Figure 3.7.: Classes of PSTH shapes

In the ICC, neurons with five distinct firing patterns in response to pure tones were encountered. (A) "Primary-like" PSTH with a sharp and strong onset response, adapting during the stimulation to a steady-state response. (B) "Pri-notch" PSTHs are similar to primary-like units, but with a prominent notch after the onset response. (C) "Tonic" PSTH shows a sustained response throughout the stimulation without a distinguishable onset response. (D) "Onset" PSTH, defined by just a sharp onset response. (E) "Offset" PSTH shows no response during the stimulation but respond directly after the offset of a stimulus.

Fig. 3.8). However, the category of "band-pass" neurons would have a slightly different meaning here. For responses to sAM tones with varying sound intensities, a "band-pass" unit would resemble more the characteristics of a unit with a peak-shaped rate-level function than of an actual response to a specific modulation frequency. Units specific for a certain modulation depth were very rare and if so, they would only be specific for $100 \%$ modulation depth and would not respond for other modulation depths.

\subsubsection{Forward masking}

Forward masking was possible to analyze for most units, independent of PSTH shapes. Therefore only the categories "normal" and "other" were introduced, to be able to exclude neurons with irregular response patterns or units that would not respond to the shortness of the probe tone, independent of the gap length (see Fig. 3.9). 


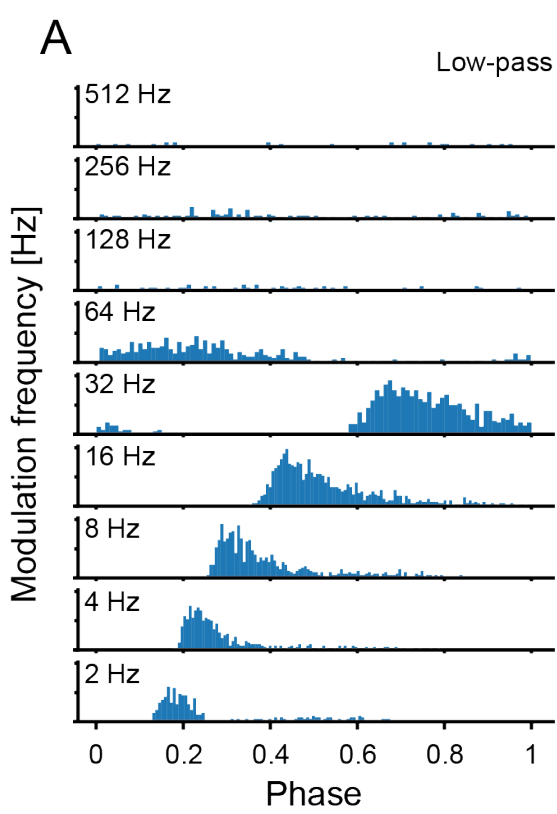

B

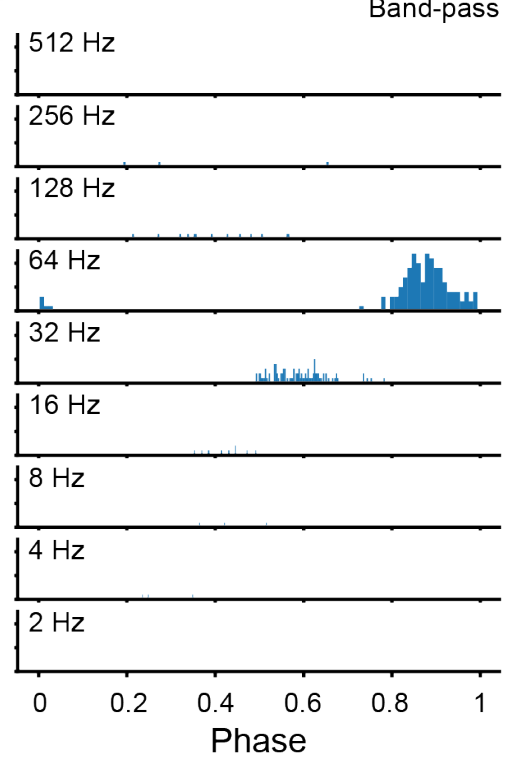

Figure 3.8.: Classes of AM tone responses

Three different response types to AM tones were discovered in ICNs. Phase-diagrams were created as described in chapter 3.5.6. (A) Phase-diagram of a "low-pass" unit, which is able to phaselock to the envelope of AM tones at low frequencies up to a certain frequency, which they cannot keep up with anymore. (B) Phase-diagram of a "band-pass" unit, which is tuned to a specific modulation frequency, responding with barely more than an onset response to frequencies other than their specific frequency.

\subsubsection{Frequency of unit types}

The frequency of all different classes for each run type is displayed in Fig. 3.10. As mentioned before, the "V-shaped" class is the most abundant FRA shape with "O-" and "I-" shaped classes about equally abundant. Non-monotonic units make about $1 / 3$ of all measured units. Moreover, the "onset" response is the most frequent response for all pure tone burst runs, independent of the tone length. In response to sAM tones the fraction of "low-pass" units is the biggest, although for the modulation intensity and modulation depth runs the distribution of unit types is slightly biased, as units with "onset" - properties for modulation frequency runs were not always recorded for the other sAM runs. All run types have a certain number of unclassifiable units assigned to the category "other". Often units could not be classified due to irregular response patterns, which change strongly during the recording of a single run. 
Figure 3.9.: Classes of forward masking responses

For most "normal" ICNs a masker and a probe response could be clearly identified and separated from each other. Units where this was not the case, or which changed their firing pattern during stimulation were excluded. The threshold for the minimal silent interval needed to elicit a probe response is marked with an arrow.
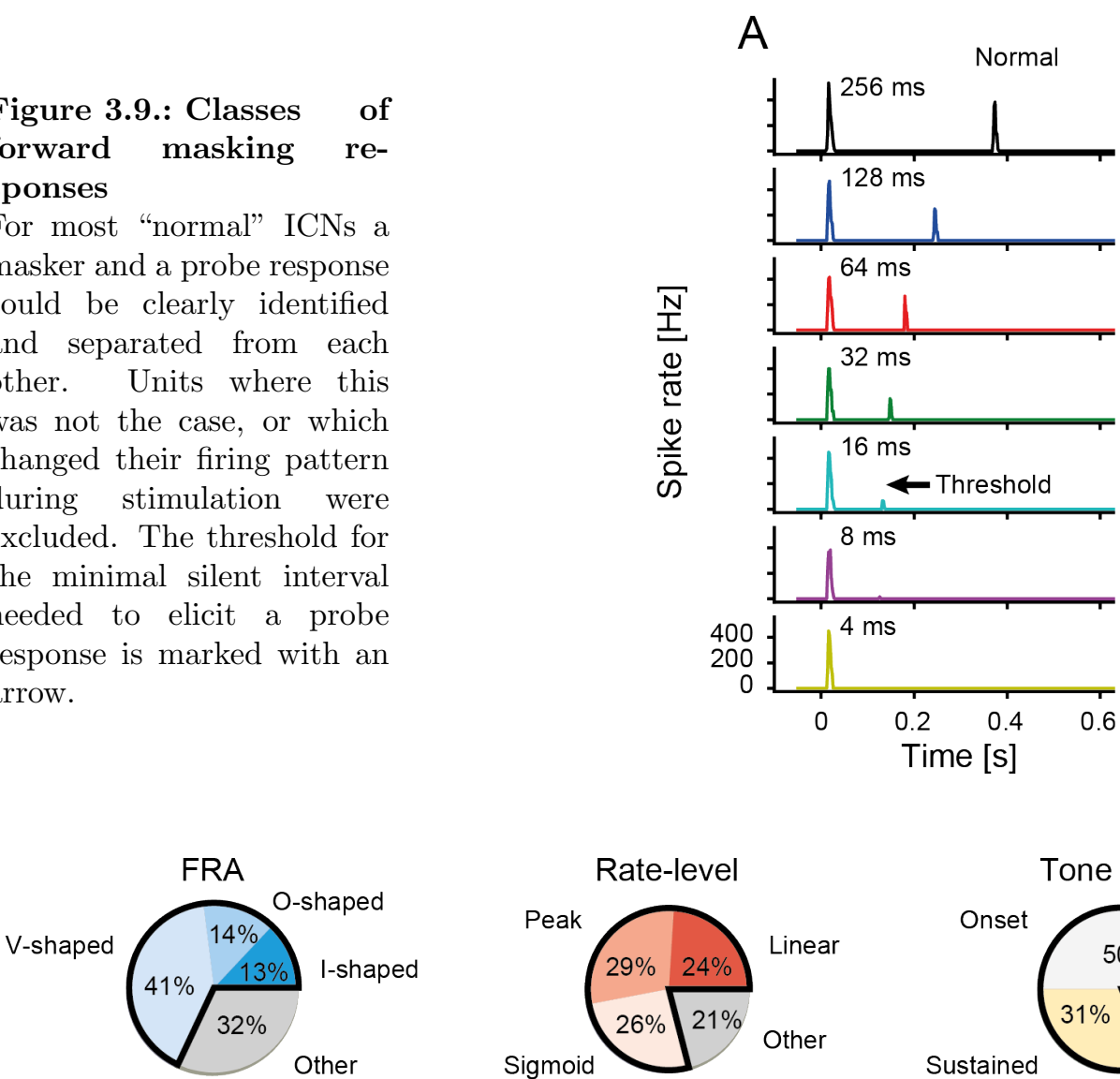

Tone burst

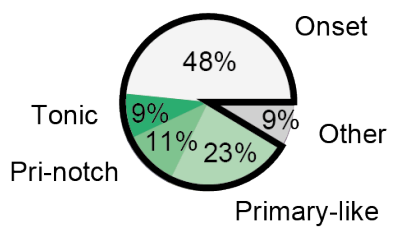

Modulation frequency

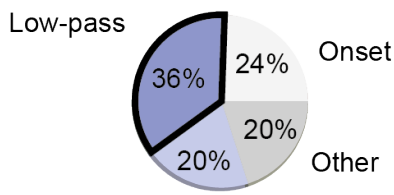

Band-pass

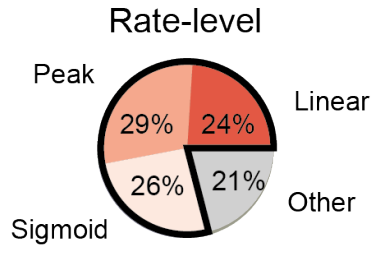

Tone burst $5 \mathrm{~Hz}$

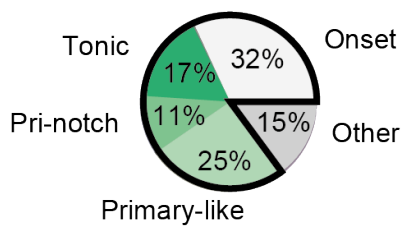

Modulation intensity

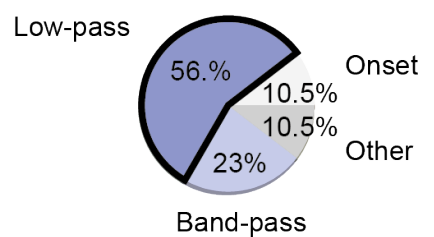

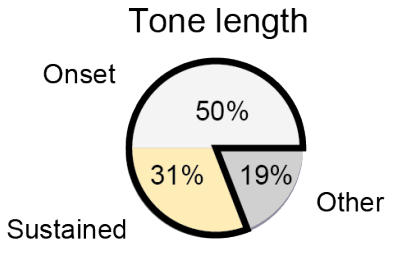

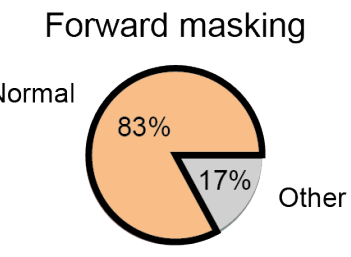

Modulation depth

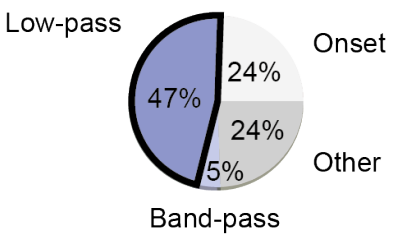

Figure 3.10.: Frequency of different classes for each run type

The frequencies of the different classes were counted for all recordings from wildtype animals to get a picture of the different response patterns that are present in the ICC. The category of "other" is comprised of units, which could not be classified into any other category. "Other"-units are excluded from all further analysis. "Offset"-units for tone burst runs (described in Fig. 3.7) were very rare and in fact only detected twice in mutant strains. Units which were used for analysis are marked with a black frame. 



\section{Results}

\subsection{Hair-cell specific bassoon knock-out mice $\left(\right.$ Bsn $^{f l / f l}$ Pres $\left.{ }^{\text {Cre }}\right)$}

Previous studies using in vivo single unit recordings from SGNs were conducted on a bassoon mouse line, where exon 4 and 5 were excised, leading to non-functional bassoon in all synapses $\left(B s n^{\Delta E x} 4 / 5\right.$ mice, see chapter 1.5.1). In these mice synaptic ribbons were reduced in number, not properly anchored to the active zone and sound evoked spiking was reduced. However, this mutation affected all synapses in the brainstem and cortex. For in vivo electrophysiological recordings from SGNs this is not problematic, as the SGNs are the first neurons in the auditory pathway. Only the IHC ribbon synapse disfunction due to lack of bassoon would affect spiking in SGNs. Interpretation of responses from ICNs of $B s n^{\Delta E \times 4 / 5}$ mice however would be difficult, as it is unknown which role bassoon plays at all synapses in the auditory brainstem.

To overcome this issue and being able to continue working with this mutation, we generated a hair-cell specific bassoon knock-out mouse line (Bsn ${ }^{f / / f l}$ Pres ${ }^{C r e}$ mice). We wanted to use this new mouse line to investigate how a peripheral defect in the IHC ribbon synapse would be processed in the brainstem by comparing ICN recordings with the previously obtained SGN data of the $B s n^{\Delta E x 4 / 5}$ mouse line.

We first wanted to confirm the bassoon deletion in the $B s n^{f / f l}$ Pres ${ }^{C r e}$ mouse line in IHCs by fluorescent immunohistochemistry. Secondly, we wanted to assess general hearing function by measuring ABRs, before starting with single unit recordings from the ICC.

\subsubsection{Immunohistochemical stainings}

We first verified that the bassoon protein was indeed deleted from the IHCs of the $B s n^{f l / f l}$ Pres ${ }^{C r e}$ mice. Therefore, we performed immunohistochemical stainings on eight organs of Corti from two cre positive and two cre negative mice at the age of 9 weeks. To begin with, we performed a double staining with an antibody against ribeye, which labels all presynaptic ribbons in the IHCs and with a sap7f-antibody labeling bassoon. Due to the expression pattern of the cre-recombinase, we expected sap7f staining in efferent fibers, but not at IHC synapses.

The number of ribbons is approximately similar in the IHCs of both mutant and wildtype stainings, shown by a comparable number and distribution of ribeye spots in Fig. 4.1, upper panels. The staining of bassoon with the sap7f-antibody was successful as well (middle panels). However, in the overlay of both channels (lower panels), a colocalization 
of the ribeye and the bassoon staining can only be seen for wildtype mice (see Fig.4.1 B), but not for $B s n^{f / / f l}$ Pres ${ }^{C r e}$ mice (see Fig.4.1 A), showing that bassoon was successfully deleted from all IHCs, but not from efferent fibers. In addition to that, bassoon was deleted from all OHCs of cre-positive mice as well (not shown).

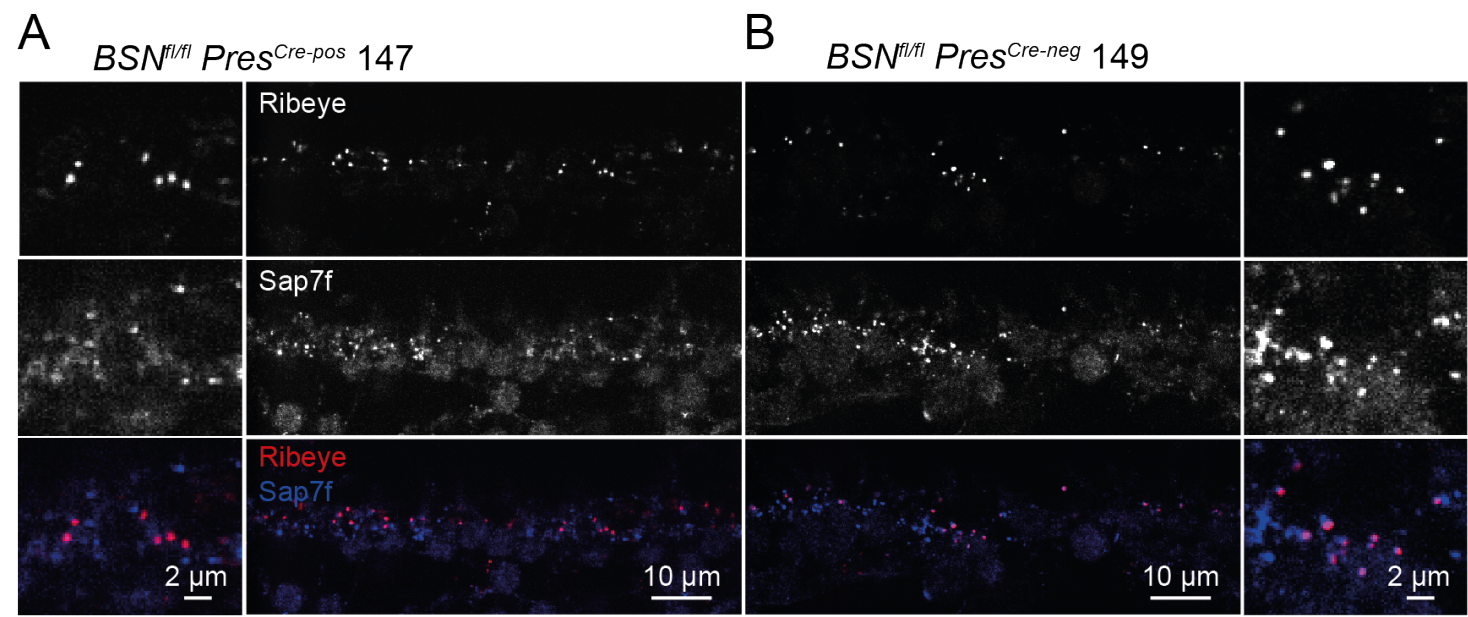

Figure 4.1.: Staining of Ribeye and Bassoon on Bsn ${ }^{f / / f l}$ Pres ${ }^{\text {Cre }}$ mice.

Representative images of immunohistochemical stainings from organs of Corti of (A) a Crepositive hair-cell specific bassoon knock-out mouse (Bsn ${ }^{f l / f l}$ Pres Cre-pos 147$)$ and (B) a wildtype littermate $\left(B s n^{f l / f l}\right.$ Pres $\left.{ }^{C r e-n e g} 149\right)$. Staining was performed with antibodies against ribeye as marker for the synaptic ribbon (upper panels) and sap7f, an antibody against bassoon (middle panels). The overlay of the wildtype staining (lower panel B) shows, that ribbons are well preserved and colocalize with bassoon. In the bassoon-knock out staining (lower panel A), ribbons are still present, but bassoon does not colocalize with ribeye. Bassoon seems to be absent from the ribbon synapses, although ribbons are formed normally. Additionally, as expected, bassoon is still expressed in efferent fibers, indicated by sap7f spots that do not colocalize with ribeye spots.

Furthermore, we performed a double staining with antibodies against ribeye and psd95 , which is a protein of the postsynaptic density. This way we wanted to address the question whether ribbons are correctly anchored to the presynaptic membrane, despite the lack of bassoon or if they are floating in the cytoplasm of the IHCs. In Fig. 4.2 it can be seen, that ribeye and psd-95 colocalize for both mutants (Fig. 4.2 A) and wildtypes (Fig. 4.2 B). Every ribeye spot is in close proximity to a psd-95 spot.

Unlike the $B s n^{\Delta E x 4 / 5}$ mouse line, which almost completely lost anchored ribbons, in this newly generated mouse line bassoon was indeed successfully knock-out, but ribbons were still intact, present in normal numbers and localized correctly at the presynapse.

\subsubsection{Auditory brainstem responses}

Hearing function of the hair-cell specific bassoon knock-out mice was assessed by measuring ABRs. Thresholds are not different between $B s n^{f l / f l}$ Pres ${ }^{C r e}$ mice and wildtype littermates (see Fig. 4.3 A) and the ABR waveform was fairly well preserved (see Fig. 4.3 B). 


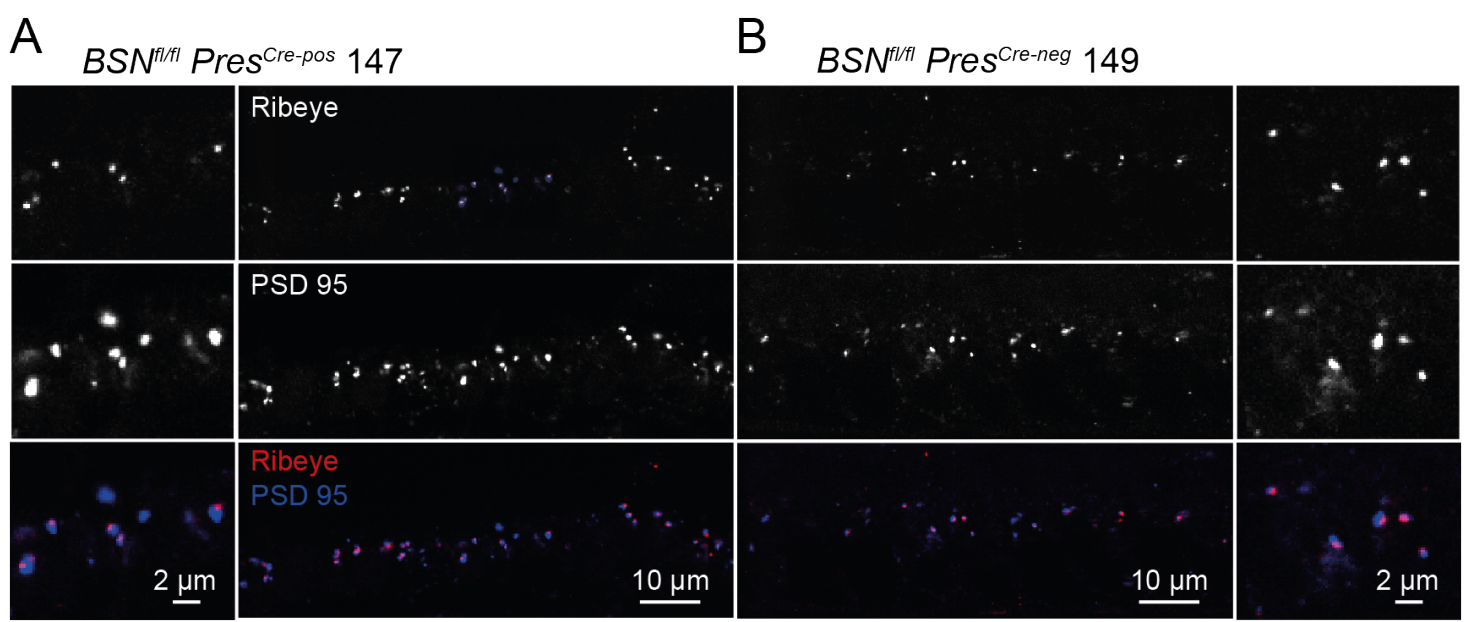

Figure 4.2.: Staining of Ribeye and PSD-95 on $B s n^{f l / f l}$ Pres ${ }^{C r e}$ mice.

Representative images of immunohistochemical stainings from organs of Corti of (A) a Crepositive hair-cell specific bassoon knock-out mouse (Bsn fl/fl Pres Cre-pos 147) and (B) a wildtype littermate $\left(B s n^{f l / f l}\right.$ Pres ${ }^{C r e-n e g}$ 149). Staining was performed with antibodies against ribeye as marker for the synaptic ribbon (upper panels) and psd-95 as postsynaptic marker (middle panels). In the overlay (lower panel) it is visible, that in both genotypes every ribbon is in close proximity to a postsynaptic density. This shows that the hair-cell specific bassoon deletion does not influence ribbon formation and localization.

ABR wave I was strongly reduced, but still visible (see Fig. $4.3 \mathrm{C}$ ) and wave III was slightly reduced. All other waves were unchanged.

To see whether changes in ABRs come from the excision of the bassoon gene or already from the expression of the cre-recombinase itself, we additionally measured ABRs from unfloxed Prestin-Cre mice. Their ABR waveform resembles that of the $B s n^{f / f l}$ Pres Cre-neg mice, indicating that the changes in ABRs indeed arise from the loss of the bassoon protein. In addition to that, we recorded ABRs from 12-14-week-old $B s n^{f l} / f l$ Pres ${ }^{C r e}$ mice to investigate if aging or potential degradation of residual bassoon would influence hearing. ABR waveforms from $12-14$-week-old $B s n^{f / f l}$ Pres ${ }^{C r e}$ mice looked similar to those from 5-6-week-old mice (not shown).

A comparison to the ABRs of the $B s n^{\Delta E x 4 / 5}$ mice (see Fig. 1.5.1) shows, that the $B s n^{f / / f l}$ Pres ${ }^{C r e}$ ABR waveform is much better preserved. Bsn ${ }^{\Delta E x 4 / 5}$ mice did not have any significant $\mathrm{ABRs}$ at $80 \mathrm{~dB}$ and had reduced and distorted waves at $100 \mathrm{~dB}$.

\subsubsection{Discussion and outlook}

The idea behind generating a hair-cell specific bassoon knock-out mouse line for single unit recordings from the ICC was to generate a similar phenotype as the $B s n^{\Delta E x 4 / 5}$ mouse line, without an influence from a lack of bassoon in all synapses of the auditory brainstem, but only from the IHC ribbon synapse. However, the hearing function of this new mouse line is not comparable with that of $B s n^{\Delta E x} 4 / 5$ mice. The bassoon knock-out was successful, but unlike in the $B s n^{\Delta E x 4 / 5}$ mice, synaptic ribbons were still present and 

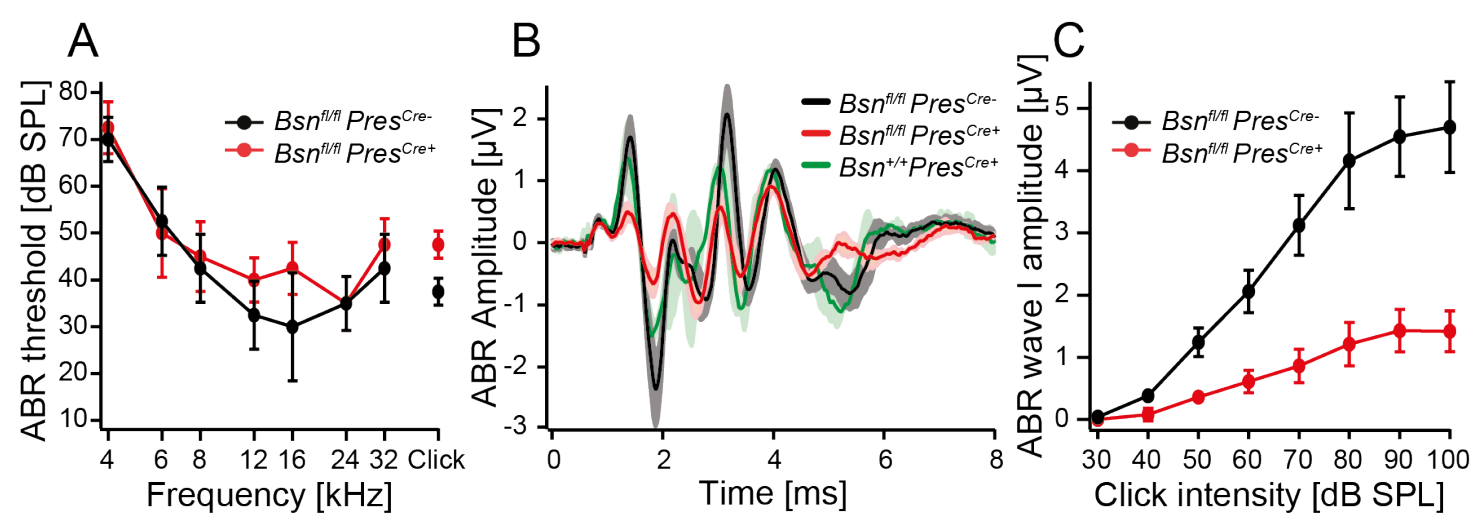

Figure 4.3.: Auditory brainstem responses of $B s n^{f l / f l}$ Pres $^{C r e}$ mice.

(A) ABR thresholds do not differ significantly between $B s n^{f l / f l}$ Pres ${ }^{C r e}$ mice $(\mathrm{n}=4)$ compared to $B s n^{f l / f l}$ Pres ${ }^{C r e-}$ littermates $(\mathrm{n}=4)$. (B) Grand averages of ABR waveform \pm SEM in response to $80 \mathrm{~dB}$ click stimulation in Bsn ${ }^{\text {fl/fl }}$ Pres ${ }^{C r e}$ mice, Bsn ${ }^{f l / f l}$ Pres ${ }^{C r e-} \quad(n=4)$ mice and unfloxed Pres ${ }^{C r e+}$ mice $(\mathrm{n}=2)$ as a further wildtype control. All mice were at the age of 5-6 weeks. ABR waves I and III are reduced while wave II and IV are normal. Unfloxed mice expressing the cre-recombinase have normal ABRs. (C) Amplitude of ABR wave I is strongly reduced in $B s n^{f l / f l}$ Pres ${ }^{C r e}$ mice at all intensities.

properly located at the presynaptic active zone. ABRs of $B s n^{f l / f l}$ Pres ${ }^{C r e}$ mice were less affected than those of $B s n^{\Delta E x 4 / 5}$ mice, indicating only mild impairments in auditory function. Especially wave IV is well preserved and wave $\mathrm{V}$ is still visible, suggesting a relatively normal spiking in the ICC.

Based on the findings obtained by analyzing the new mouse several new questions arise. Bassoon was thought to anchor the ribbons to the presynaptic membrane, but our immunohistochemical staining show that a proper localization might be possible without bassoon. Knowing the expression profile of the prestin protein under whose promotor the cre-recombinase is expressed, bassoon is likely deleted from p5 on. Possibly, an expression of bassoon before p5 suffices to anchor the ribbons to the membrane. To check whether the ribbons are properly anchored to the presynaptic membrane or just localized at the right spot, a proximity analysis between ribeye and psd-95 spots in the immunohistochemical stainings and electron microscopic pictures would be of interest.

Although these would be interesting questions to address, they're not in line with the original research question, to investigate the coding of auditory signals in the ICC, in this study. As the $B s n^{\Delta E \times 4 / 5}$ mice have different properties from the $B s n^{f l / f l}$ Pres ${ }^{C r e}$ mice, the previously obtained SGN recording data cannot be compared directly to recordings from the ICC of the Bsn ${ }^{f / f l}$ Pres ${ }^{C r e}$ mice. This would mean all single unit recordings from SNGs would have to be repeated on the $B s n^{f l / f l}$ Pres ${ }^{C r e}$ mice. Still, it is questionable whether recordings from the ICC of these mice would lead to major insights into auditory coding in the ICC. As the ABRs were only mildly affected and ABR wave IV and V were least impaired it is doubtful that huge impact of this knock-out on the ICC could be observed at all. 
In summary, this newly generate hair-cell specific bassoon knock-out mouse line could turn out to be useful in studying the function of the ribbon synapse in terms of ribbon anchoring, but less in studying sound encoding in the ICC.

\subsection{Otoferlin Ile515Thr mice (Otof ${ }^{I 515 T / I 515 T}$ )}

Otoferlin is a hair-cell specific protein with suggested functions for a number of essential features, required for hearing like exocytosis of synaptic vesicles, vesicle priming or vesicle reformation and replenishment. Mutations in otoferlin are particularly interesting for this project, as they will only affect sound transmission at the IHC ribbon synapse. This way effects of a pure peripheral defect can be analyzed regarding the question how these defects are propagated throughout the auditory pathway. For this purpose we are using in vivo single unit recordings from the ICC of Otof ${ }^{I 515 T / I 515 T}$ mice. With these we want to find out whether and which sound features the ICC can compensate compared to the SGNs and how the defects observed in the SGNs are propagated throughout the brainstem. Furthermore, we would like to find out why speech perception of the human patients carrying an $O T O F^{I 515 T / I 515 T}$ mutation is so poor, despite normal hearing thresholds.

In vivo single unit recordings from the ICC were performed on 14 Otof ${ }^{I 515 T / I 515 T}$ mice and 13 Otof $^{+/+}$littermates. Analysis of the single neurons includes general properties, like spontaneous and sound evoked spike rates, as well as spike timing and phase-locking to the envelope of sAM tones. Moreover, the ability to detect silent gaps between tones and the propagation of vesicle replenishment defects is analyzed by forward masking.

\subsubsection{Tuning, thresholds and spontaneous rates are normal in ICNs of Otof ${ }^{I 515 T / I 515 T}$ mice}

Firstly, we investigated structural organization in the ICC, regarding distributions of CFs, spontaneous rates or thresholds.

Similar to the organization in the SGNs, the neuronal architecture is unchanged in the ICC of Otof ${ }^{I 515 T / I 515 T}$ mice. Distribution of spontaneous rates was comparable between wildtype and mutant mice (see Fig. 4.4 A), however, spontaneous rate in ICNs of anesthetized animals is very low in general. Distribution of CFs was comparable between genotypes as well and correlated with the unit depth (see Fig. $4.4 \mathrm{~B}$ ), the depth of the electrode where a specific unit was encountered. This shows an intact tonotopical organization, confirming that the recording site was indeed the ICC. Pure tone thresholds from single units of the ICC did not differ between genotypes as well (see Fig. 4.4 C), but distribution of optimal intensities was significantly lower (see Fig. 4.4D). However, it is very unlikely that this shows an impaired neuronal organization in the ICC in favor of better intensity coding, but more likely that this is due to a higher fraction of "peak"shaped units sampled in Otof ${ }^{I 515 T / I 515 T}$ mice than in wildtype littermates. All in all, the organization in the ICC is still intact and no loss of a specific fraction of fibers could be observed in Otof ${ }^{1515 T / I 515 T}$ mice. 


\section{A}

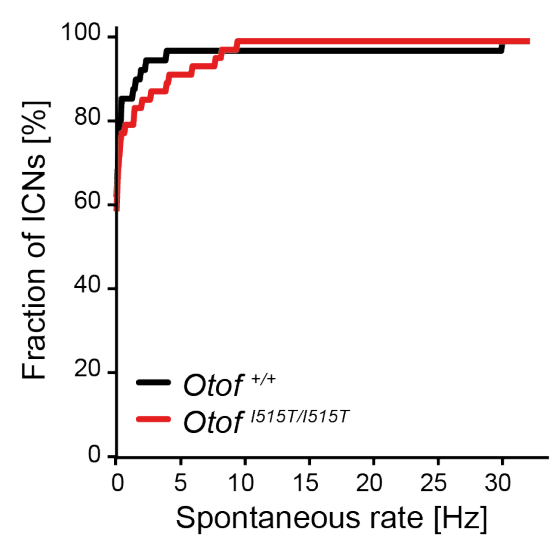

C

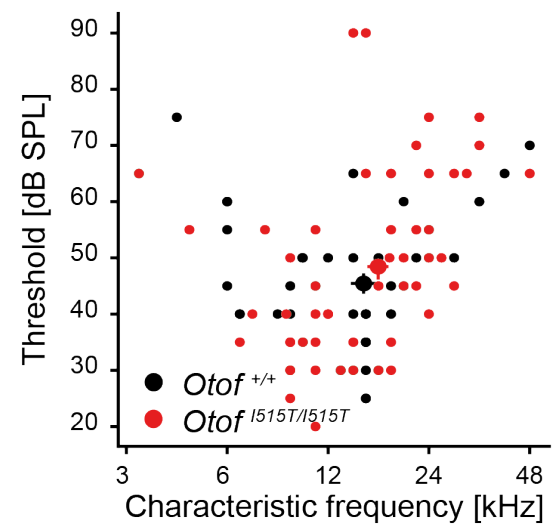

$B$

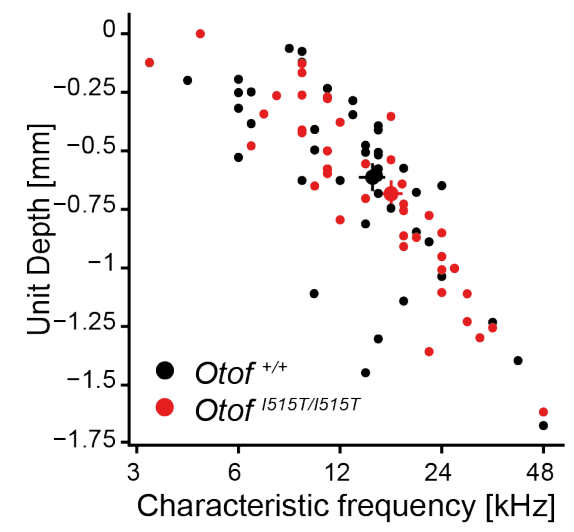

D

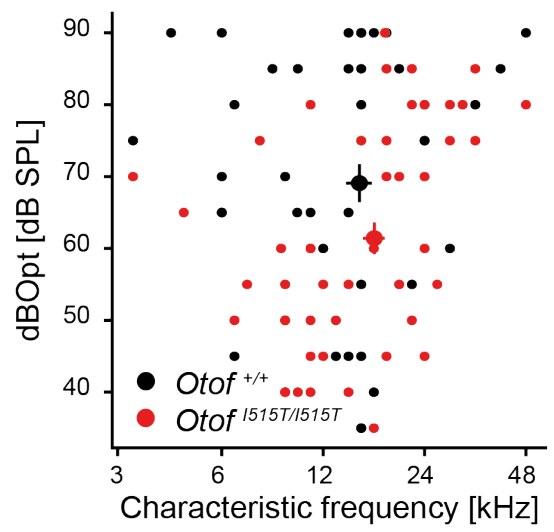

Figure 4.4.: Spontaneous rate, tuning and thresholds are normal in Otof ${ }^{1515 T / I 515 T}$ mice.

(A) Cumulative histogram of the spontaneous rate of all ICNs from Otof I515T/I515T mice (n $=55)$ and wildtype littermates $(\mathrm{n}=48)$. Spontaneous rate was generally low for excitatory single units from the ICC and did not differ between wildtype and mutant neurons (KolmogorovSmirnov-test, $\mathrm{p}=0.7$ ). (B) Depth of the recording site in the brain as a function of the CF. The unit depth correlates with the CF for both wildtype $(\mathrm{n}=43)$ and mutant $(\mathrm{n}=42)$ ICNs, showing an intact tonotopy and proving, that the recording site was the ICC. (C) Threshold of each unit plotted against CF. Thresholds were normal in ICNs from Otof ${ }^{I 515 T / I 515 T}$ mice $(\mathrm{n}=$ 49) compared to wildtype littermates $(\mathrm{n}=44)$. (D) dBOpt of each unit plotted against CF. The average dBOpt of mutant units $(\mathrm{n}=51)$ was lower than the one of wildtype units $(\mathrm{n}=$ 46; Mann-Whitney-U-test, $\mathrm{p}=0.02$ ). This can be explained by a higher fraction of measured "peak"-responsive units for Otof ${ }^{1515 T / I 515 T}$ mice. (B-D) Big circles represent mean values \pm SEM. The full statistical analysis is shown in appendix B.

\subsubsection{Intensity coding is impaired in terms of a reduction in dynamic range}

Next, we wanted to analyze the effect of the Otof ${ }^{I 515 T / I 515 T}$ mutation on intensity coding and rate-level functions of ICNs. Unlike in SGNs, not all rate-level functions of ICNs are 
monotonic and sigmoid shaped, so we divided all ICNs into a group of monotonic and a group of non-monotonic fibers, to be able to calculate the dynamic range (see 3.5.3).

In general the shapes of rate-level functions of both fiber types between wildtype and mutant mice were similar (see Fig. 4.5 A, D). Unlike in SGNs, a comparison of maximal and mean spike rates did not reveal any significant differences, neither for monotonic (see Fig. 4.5 B) nor for non-monotonic fibers (see Fig. 4.5 E). However, dynamic range was significantly smaller for both monotonic (see Fig. $4.5 \mathrm{C}$ ) and non-monotonic fibers (see Fig. 4.5 F).
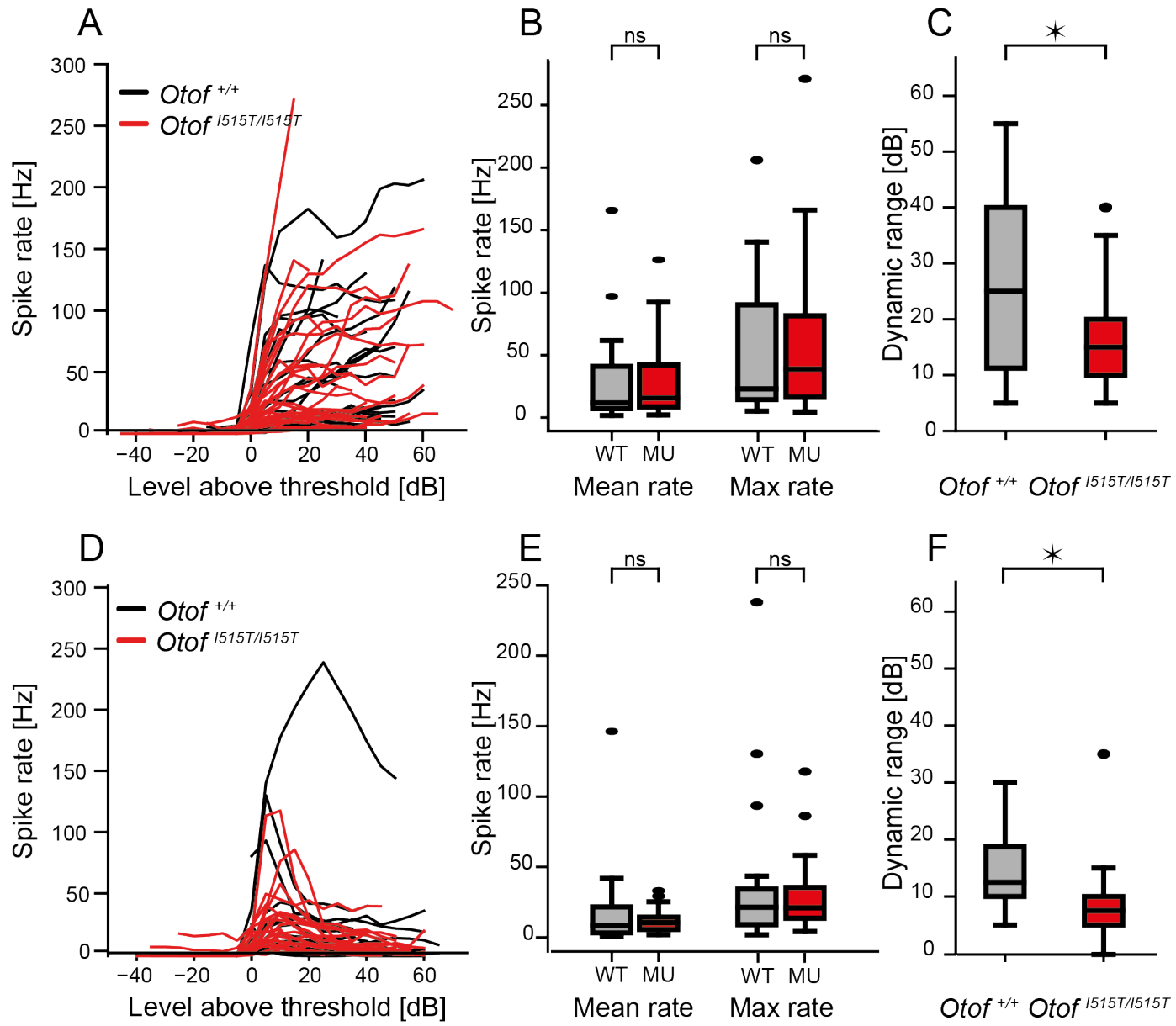

Figure 4.5.: Rate-level functions from ICNs of Otof $^{1515 T / I 515 T}$ mice have a smaller dynamic range.

Rate-level functions of all units with a monotonicity index (A) higher than 0.75 and (D) lower than 0.75 , plotted relative to their threshold. Mean and maximal spike rates did not differ between $(\mathrm{B})$ wildtype $(\mathrm{n}=30)$ and mutant mice $(\mathrm{n}=30)$ for monotonic units and $(\mathrm{E})$ wildtype $(\mathrm{n}=18)$ and mutant mice $(\mathrm{n}=24)$ for non-monotonic units. Dynamic range was significantly smaller for ICNs of Otof ${ }^{1515 T / I 515 T}$ mice compared to wildtype littermates for (C) monotonic single units (Kruskal-Wallis-test, $\mathrm{p}=0.02$ ) as well as for $(\mathrm{F})$ non-monotonic single units (MannWhitney-U-test $\mathrm{p}=0.04)$. The full statistical analysis is shown in appendix B. 
In summary, an impairment described by reduced maximal spike rates in SGNs was changed in the ICNs into a reduction in dynamic range with normal spike rates.

\subsubsection{Coding of short tones is unaffected by the Otof I515T/I515T $^{I 5}$ mutation}

We wanted to investigate duration tuning in the ICC of Otof ${ }^{1515 T / I 515 T}$ mice. However, we were unable to find ICNs coding specifically for a certain tone length in this study, neither in wildtype mice, nor in mutant mice. Here, we were only able to distinguish between neurons that respond throughout the stimulation, dependent on the tone length ("sustained" fibers) and neurons that respond at the beginning of the tones, independent of the tone length ("onset" fibers). However, not all units were able to respond to the shortest tones, so we determined the minimal tone length needed to elicit a response for each neuron.

The distribution of minimal tone length was comparable between ICNs of Otof I515T/I515T mice and Otof $^{+/+}$littermates (see Fig. 4.6 A). Having a closer look on the different classes of neurons showed similar thresholds between "onset" (Fig. 4.6 B) and "sustained" units (Fig. 4.6 B) and did not reveal any differences between mutant and wildtype ICNs in the coding of short tones.
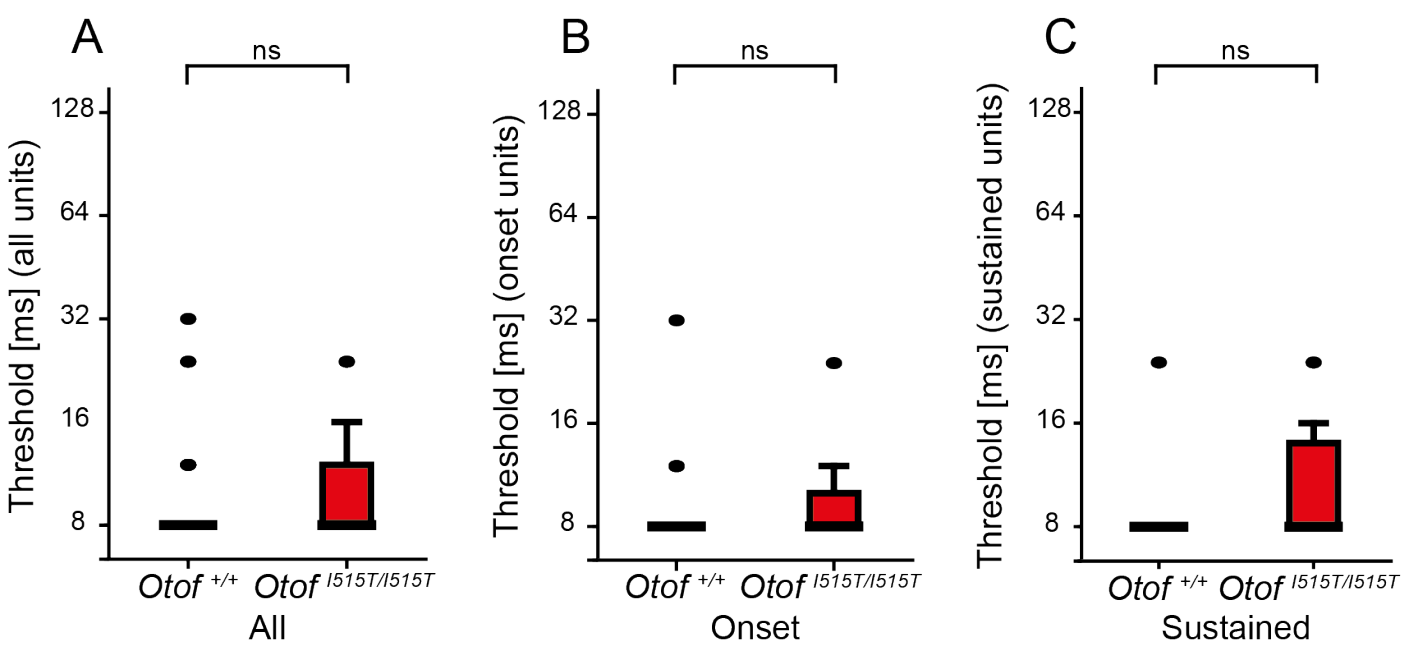

Figure 4.6.: Responses to pure tones with different tone length are normal in ICNs from Otof ${ }^{I 515 T / I 515 T}$ mice.

(A) The average minimal tone length, which is needed to elicit a response did not differ between ICNs of Otof ${ }^{1515 T / I 515 T}$ mice $(\mathrm{n}=28)$ and wildtype littermates $(\mathrm{n}=24)$. A separate analysis of (B) "onset"-type neurons (wildtype $\mathrm{n}=17$, mutant $\mathrm{n}=11$ ) and $(\mathrm{C})$ "sustained"-type neurons (wildtype $\mathrm{n}=7$, mutant $\mathrm{n}=11$ ) did not reveal any deficits in the coding of short tones for Otof $^{I 515 T / I 515 T}$ mice. The full statistical analysis is shown in appendix B. 


\subsubsection{Pure tone responses have a reduced and more jittered onset response}

Next, we wanted to investigate the responses of ICNs from Otof ${ }^{I 515 T / I 515 T}$ mice to pure tone stimulation and were analyzing spike rates and spike timing. PSTH shapes in the ICC are much more diverse than in the AN. However, when averaging all responses from all ICNs, the resulting PSTH possesses a "primary-like" shape like the SGNs as well (compare Fig. 4.7 D).

To quantify the differences in spike rate, we calculated the peak and steady-state rate for each neuron. To visualize these rates we created an averaged peak-aligned PSTH (see Fig. 4.7 A). For this, onset peaks from all neurons were all aligned on the time scale. In ICNs from Otof ${ }^{I 515 T / I 515 T}$ mice, the averaged peak rate was significantly smaller (see Fig. $4.7 \mathrm{~B}$ ). For the quantification of steady-state rates all "onset-" type units, which do not possess a steady-state rate, were excluded from the analysis. Unlike in SGNs, we don't see a reduction in steady-state rate of ICNs, however, the steady-state rate is generally low (see Fig. $4.7 \mathrm{C}$ ).

Moreover, we were interested in spike timing of ICNs. To visualize precision of spike timing and get an impression of the overall response over time of ICNs we created a PSTH without peak-alignment (see Fig. 4.7 D). Similar to spike timing in SGNs, median FSL is normal in ICNs of Otof ${ }^{1515 T / I 515 T}$ mice (see Fig. 4.7 E), but more jittered (see Fig. $4.7 \mathrm{~F}$ ). This increase in variance of FSL might explain the reduced peak rate observed in Fig. 4.7 B.

In addition to that, we were trying to fit a single- or double-exponential function to the PSTH time course to calculate time constants for adaptation. However, the heterogeneity of responses from single neurons was too high to return convincing fits and time constants.

In summary, spike rates seem to be only mildly affected by the Otof ${ }^{I 515 T / I 515 T}$ mutation. A small reduction in onset spike rate is visible, which correlates with a slightly increased variance of first spike latencies. A stronger adaptation or a reduction in steady state rate could not be shown in ICNs of Otof ${ }^{I 515 T / I 515 T}$ mice. 

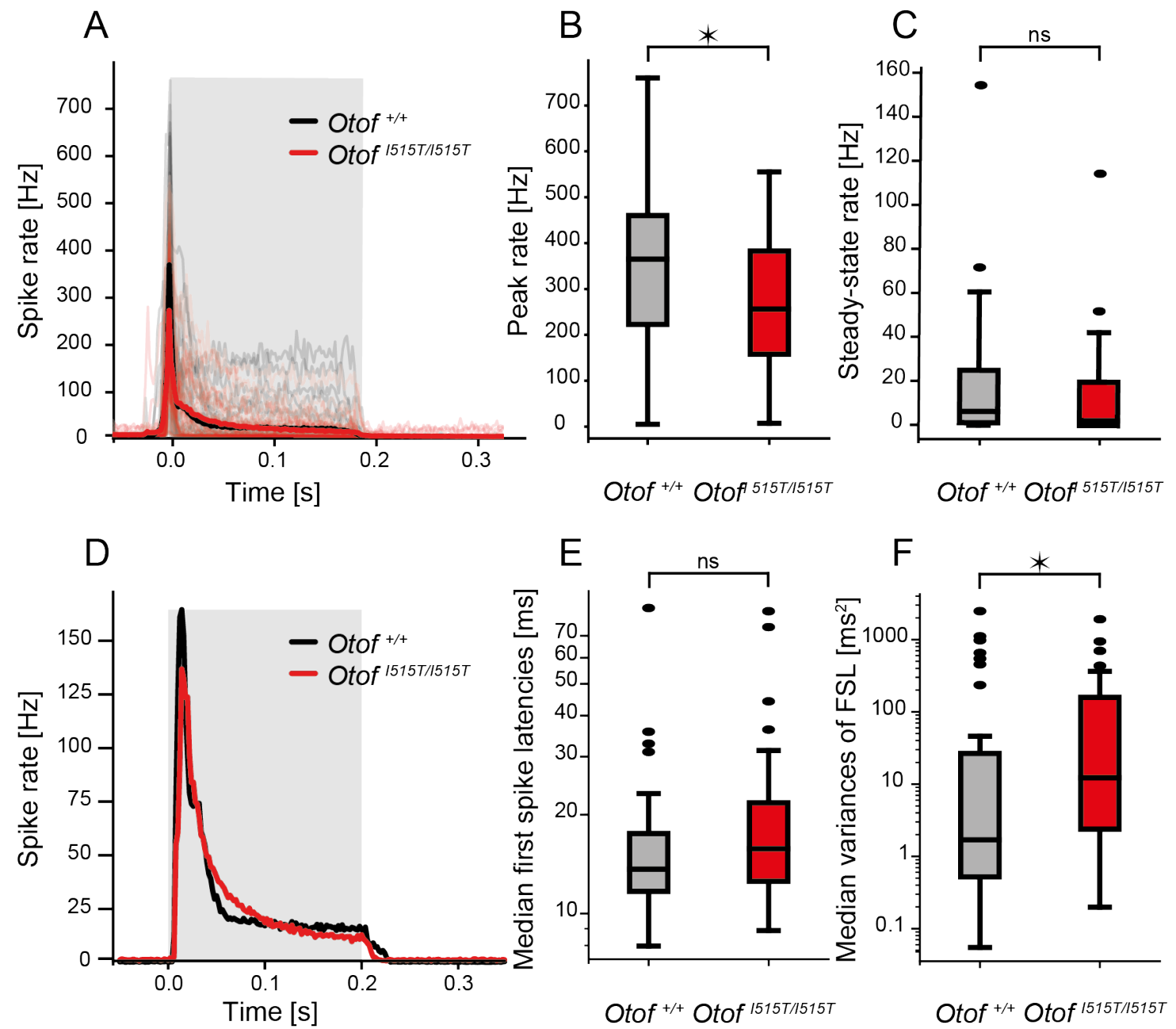

Figure 4.7.: Onset responses are reduced and more jittered in ICNs of Otof ${ }^{I 515 T / I 515 T}$ mice.

(A) Averaged peak-aligned PSTH of wildtype $(\mathrm{n}=41)$ and mutant $(\mathrm{n}=44)$ ICNs. The time when the stimulus was presented is colored in grey. (B) Quantification of the peak rate. The onset response is significantly reduced in mutant ICNs (T-test, $\mathrm{p}=0.008$ ). (C) For quantification of the steady-state rate all "onset"-type neurons, which do not have any steady-state response were excluded. The steady-state rate did not differ between ICNs of Otof ${ }^{I 515 T / I 515 T}$ mice $(\mathrm{n}=16)$ and wildtype littermates $(\mathrm{n}=26)$. (D) Averaged PSTH of wildtype and mutant ICNs without peak alignment. The time when the stimulus was presented is colored in grey. (E) Median FSL was normal in ICNs of Otof $f^{I 515 T / I 515 T}$ mice compared to wildtype littermates. (F) Variance of FSL was increased in Otof ${ }^{1515 T / I 515 T}$ ICNs (Mann-Whitney-U-test, $\mathrm{p}=0.013$ ) consistent with the slightly reduced onset rate shown in (B). The full statistical analysis is shown in appendix B. 


\subsubsection{Forward masking is impaired in ICNs of Otof ${ }^{I 515 T / I 515 T}$ mice}

Furthermore, we were interested how a defect in forward masking, which was present in SNGs of Otof ${ }^{I 515 T / I 515 T}$ mice, observed as an increased depression of spiking and a delayed recovery, is propagated throughout the brainstem and processed in the ICC. Therefore we performed the same forward masking paradigm with the presentation of a 100 ms masker tone, followed by a silent interval between $4-256 \mathrm{~ms}$ and a $15 \mathrm{~ms}$ probe tone. Here we show no compensation for the depression of spiking (see Fig. 4.8 A), which is also increased in ICNs of Otof ${ }^{I 515 T / I 515 T}$ mice compared to Otof ${ }^{+/+}$littermates at gap length between 32 and $256 \mathrm{~ms}$. Furthermore, the minimal silent interval needed to elicit any probe response was increased in ICNs from Otof ${ }^{I 515 T / I 515 T}$ mice (see Fig. 4.8 B).
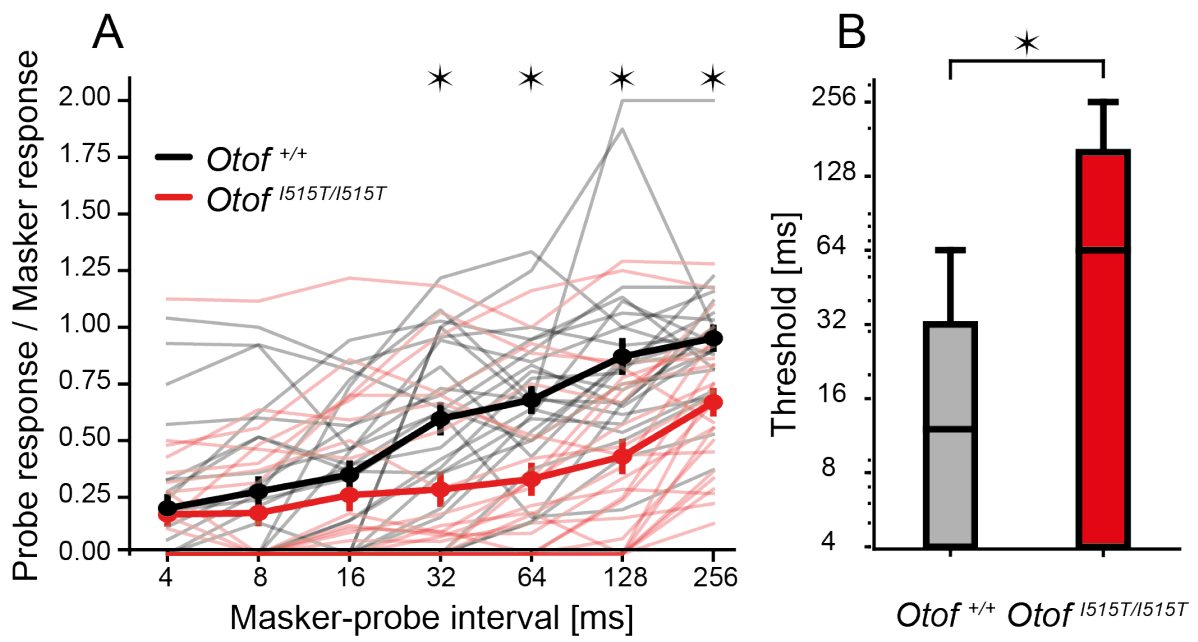

Figure 4.8.: Depression of spiking in a forward masking paradigm is impaired in ICNs of Otof ${ }^{I 515 T / I 515 T}$ mice.

(A) Depression of spiking, measured by a ratio between spike rates of probe and masker responses, is strongly increased in ICNs of Otof ${ }^{I 515 T / I 515 T}$ mice $(\mathrm{n}=24)$ compared to wildtype littermates $(\mathrm{n}=24)$ at gap length between $32-256 \mathrm{~ms}$. At lower gap length many, but not all wildtype units fail to respond to the probe tone as well. Circles represent mean \pm SEM, asterisk mark significant differences $(\mathrm{p}<0.05)(\mathrm{B})$ The minimal silent interval needed to elicit a probe response is increased in ICNs of Otof ${ }^{I 515 T / I 515 T}$ mice compared to wildtype littermates (Kruskal-Wallistest, $\mathrm{p}=0.026)$. The full statistical analysis is shown in appendix B.

\subsubsection{Phase-locking to amplitude modulated tones is normal, but spike rates are strongly reduced, and adaptation is increased}

Furthermore, we were interested in the influence of the Otof ${ }^{1515 T / I 515 T}$ mutation on phase-locking and envelope coding in response to sAM tones in ICNs. As it is known that the ICC is not capable of phase-locking up to very high frequencies we only tested modulation frequencies between 2 and $512 \mathrm{~Hz}$. For each "low-pass" single unit from the ICC, the phase and the vector strength were calculated for each tested modulation frequency. 
To illustrate these measuring units, we created a circle diagram for each modulation frequency up to $256 \mathrm{~Hz}$ (see Fig. 4.9). The resulting vectors get shorter the higher the modulation frequency is, until they end already almost in the middle of the circle at $256 \mathrm{~Hz}$ modulation frequency, which means the phase-locking becomes worse with higher modulation frequencies. ICNs were not able to phase-lock the highest measured frequencies any more. The orientation of the vectors shifts slightly from graph to graph, which is in line with the cycles getting shorter the higher the modulation frequency gets. The time when most spikes occur stays approximately the same, in absolute time dimensions from the beginning of each cycle. Surprisingly, the resulting vectors representing ICNs from Otof ${ }^{I 515 T / I 515 T}$ mice are very much comparable to those of Otof ${ }^{+/+}$littermates and phase-locking to the envelope of sAM tones seems to be normal in the ICC of Otof ${ }^{I 515 T / I 515 T}$ mice.
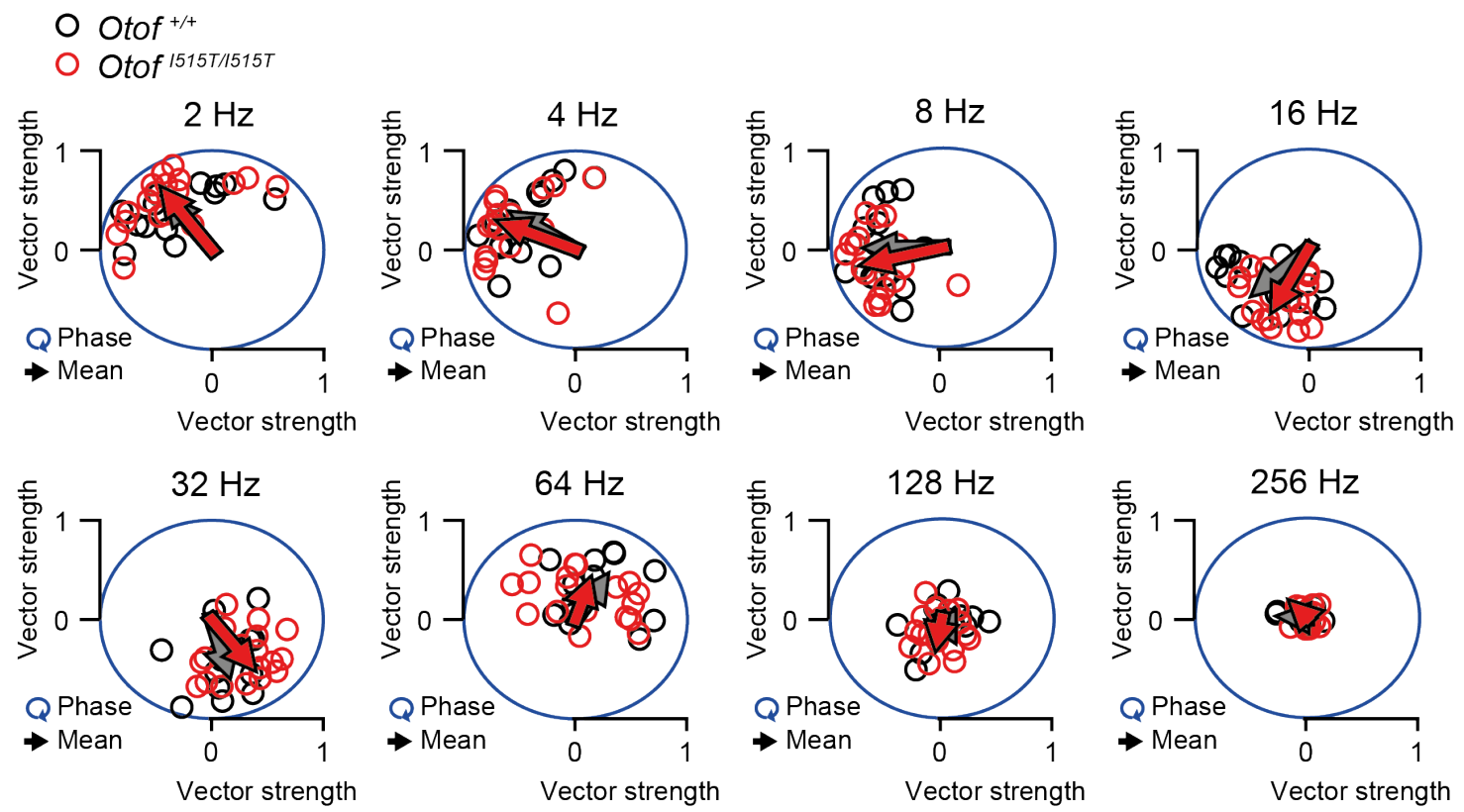

Figure 4.9.: Circle diagrams to depict phase-locking to amplitude modulated tones in ICNs from Otof $1515 T / I 515 T$ mice.

Circle diagrams of vector strength and phase plotted for each modulation frequency. The phase corresponds to the position on the circumference while the vector strengths corresponds to the position on the radius. The farther outside, the higher is the vector strength. The corresponding vectors from all wildtype and mutant single units show similar length and orientation for each modulation frequency, indicating a normal phase-locking for ICNs of Otof ${ }^{I 515 T / I 515 T}$ mice.

Yet, when looking at the PSTHs for every modulation frequency (see Fig. 4.10 A) a clear difference can be seen between wildtype (black) and mutant (red) traces. While at low modulation frequencies the PSTH shapes are similar between genotypes, at intermediate modulation frequencies, especially at $32-64 \mathrm{~Hz}$ we can observe a strong reduction of spike rate for ICNs from Otof ${ }^{1515 T / I 515 T}$ mice.

Quantification of the absolute spike rates averaged over the whole $1 \mathrm{~s}$ long stimulation shows are reduction between $8-256 \mathrm{~Hz}$ (see Fig. 4.10 B). Moreover, the adaptation 
throughout the stimulation, measured by a decay ratio is strongly increased between 4$64 \mathrm{~Hz}$ modulation frequency (see Fig. $4.10 \mathrm{C}$ ). In addition to that, we calculated the failure rate throughout the stimulation, which was significantly higher in ICNs of Otof ${ }^{\text {I515T/I515T }}$ mice and correlated with the modulation frequency (see Fig. 4.10D). This shows that ICNs are not able to respond with a spike to every phase of a stimulus above $16-32 \mathrm{~Hz}$ anymore, while ICNs of Otof ${ }^{I 515 T / I 515 T}$ mice already have difficulties to respond to every phase at $8-16 \mathrm{~Hz}$ modulation frequency.

A

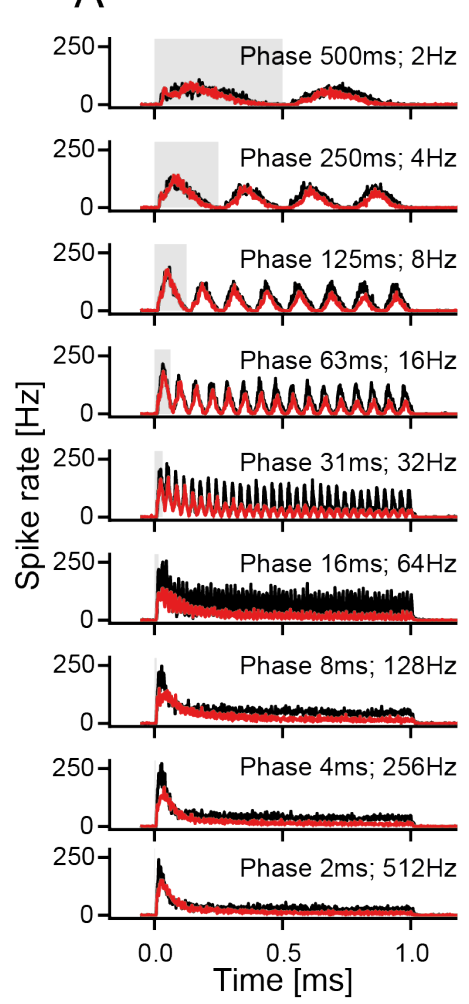

B

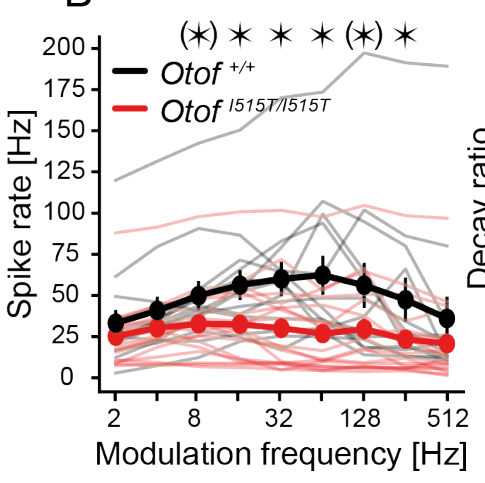

D

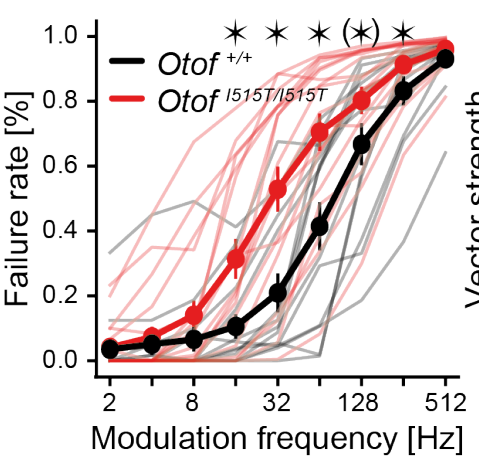

C

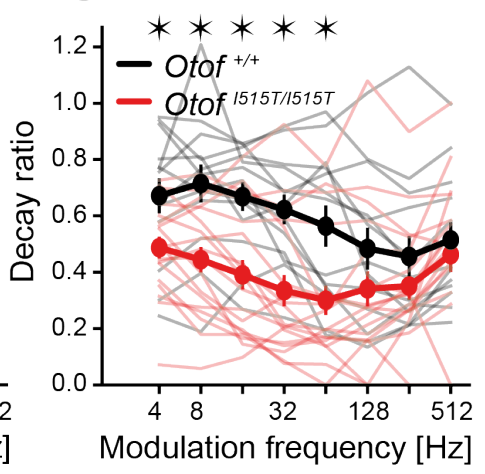

$\mathrm{E}$

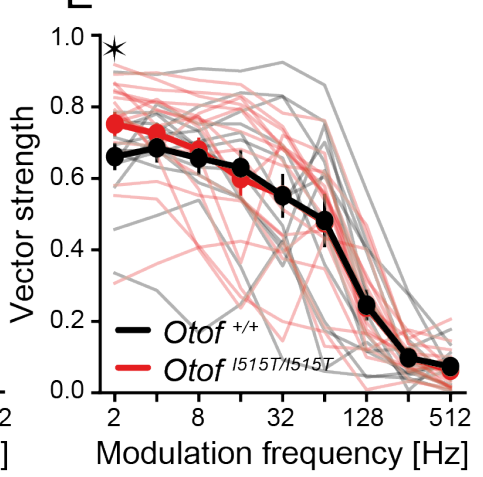

Figure 4.10.: Responses to amplitude modulated tones with varying modulation frequencies reveal a normal precision of spike timing, but a strong decline in spike rate for ICNs of Otof ${ }^{1515 T / I 515 T}$ mice.

(A) Averaged PSTHs for each modulation frequency separately from $2-512 \mathrm{~Hz}$ at dBOpt. Especially at middle frequencies a strong depression of spiking can be seen for ICNs of Otof I515T/I515T mice $(\mathrm{n}=13)$ compared to wildtype littermates $(\mathrm{n}=18)$. Duration of one cycle is marked in grey. (B) Rate modulation transfer function, which plots the averaged spike rate throughout the whole $1 \mathrm{~s}$ stimulation against the modulation frequency. Spike rates are reduced over most modulation frequencies in Otof ${ }^{I 515 T / I 515 T}$ ICNs. (C) The decay ratio, expressing the adaptation over time during amplitude modulated tones, was strongly decreased, especially at lower modulation frequencies. (D) Failure rate plotted against modulation frequency. Comparable to the reduced spike rate the failure rate was increased, especially for higher modulation frequencies. (E) Synchronization modulation transfer function, which shows the vector strength as a measure of phase-locking plotted against modulation frequency, shows a normal precision of spike timing. Circles in (B-D) represent mean \pm SEM. Asterisk represent significant differences $(\mathrm{p}<0.05)$ while asterisk in brackets represent no significance, but a difference which is still highly probable (p $<0.07)$. The full statistical analysis is shown in appendix B. 

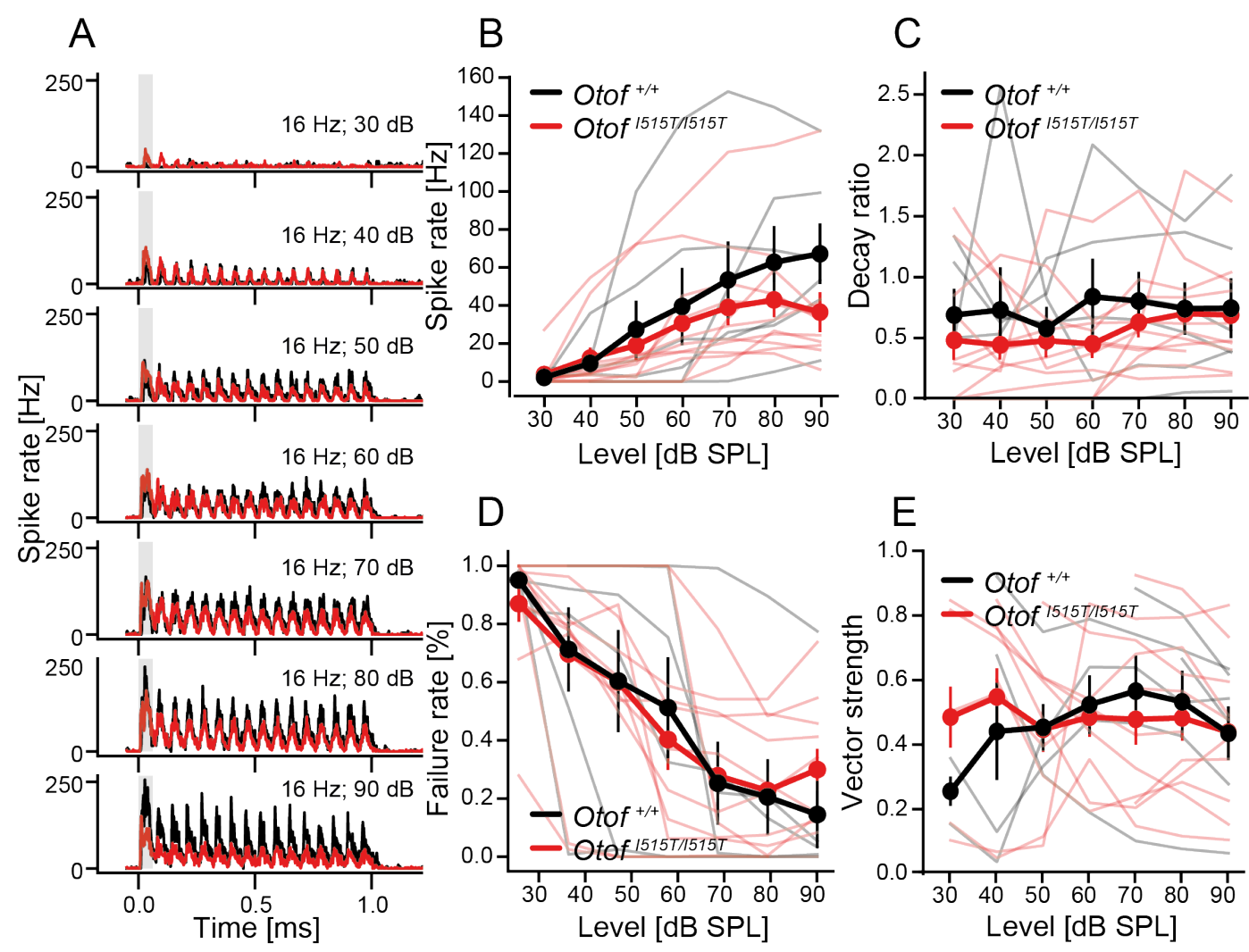

Figure 4.11.: Responses to amplitude modulated tones with varying sound intensities might have a moderate effect on ICNs of Otof $^{1515 T / I 515 T}$ mice.

(A) Averaged PSTHs for each tested sound intensity at $16 \mathrm{~Hz}$ modulation frequency, separately from 30-90 $\mathrm{dB}$ for ICNs of Otof ${ }^{I 515 T / I 515 T}$ mice $(\mathrm{n}=11)$ and wildtype littermates $(\mathrm{n}=6)$. Duration of one cycle is marked in grey. (B) Rate modulation transfer function, which plots the averaged spike rate throughout the whole $1 \mathrm{~s}$ stimulation against the modulation frequency. Spike rates are not significantly different, but it seems that wildtype ICNs increase their spike rate on average with rising intensity, while this might be less pronounced in ICNs of Otof ${ }^{1515 T / I 515 T}$ mice. (C) The decay ratio, expressing the adaptation over time during amplitude modulated tones, does not change according to the sound level. (D) The failure rate correlates with the sound intensity as it is dropping linearly with rising sound levels. (E) Synchronization modulation transfer function, which shows the vector strength as a measure of phase-locking plotted against modulation frequency, shows a normal precision of spike timing. (B-E) No significant differences could be seen in this stimulation paradigm.

Circles in (B-D) represent mean \pm SEM. The full statistical analysis is shown in appendix B.

To have a closer look on the phase-locking abilities of the ICC in general, we plotted the vector strength of each unit against its corresponding modulation frequency (see Fig. 4.10E). Here we can see the vector strength slightly decreasing with higher modulation frequencies until a sharp drop between $64-128 \mathrm{~Hz}$ which seems to be the border up to which frequency the ICNs are able to phase-lock to the envelope of sAM tones. Like shown before in Fig. 4.9, the phase-locking capabilities are similar between ICNs of Otof ${ }^{I 515 T / I 515 T}$ mice and to Otof ${ }^{+/+}$littermates. 
To further investigate how the ICC processes sAM tones, we also recorded responses to sAM tones with a modulation frequency of $16 \mathrm{~Hz}$ and varying sound intensities between 30-90 dB SPL, as well as sAM tones with a modulation frequency of $16 \mathrm{~Hz}$ and varying modulation depths between $0-100 \%$ at dBOpt.

As these recordings were the last ones obtained in the stimulus protocol (compare chapter 3.4.2), the number of successful recordings from neurons which lasted up to here was rather small. We can get a good impression on how the ICC processes these kinds of sAM tones, but we can only make assumptions on how the ICNs of Otof ${ }^{I 515 T / I 515 T}$ mice differ from those of wildtype littermates, as statistical test will fail to show significant differences for groups with too few recordings.

In the averaged PSTH for each tested sound intensity (see Fig. 4.11 A) we see that the spike rate increases with increasing sound intensity, while the precision of spiking seems to stay the same. By looking at the PSTH and the rate-modulation transfer function (see Fig. 4.11 B), it seems like the spike rate rises less in Otof ${ }^{I 515 T / I 515 T}$ ICNs with increasing sound intensity than in ICNs of wildtype littermates. The adaptation, measured by the decay ratio seems to be not dependent on sound intensity as it does not change with rising sound levels (see Fig. $4.11 \mathrm{C}$ ). Although the spike rate increases with sound intensity, the onset and adapted rates of the stimulation seem to increase by a similar amount. The failure rate (see Fig. 4.11D) correlates strongly with sound intensity with a 90-100\% failure rate at $30 \mathrm{~dB}$ and a less than $20 \%$ failure rate at $80-90 \mathrm{~dB}$, but a difference between wildtype and mutant ICNs could not be observed. The phase-locking seems to be unaffected by sound intensity (see Fig. 4.11 E), however note, that the calculation of vector strength is less accurate for low spike numbers at low sound intensities.

Similar to the PSTH in response to sAM tones with varying sound intensities, it seems the spike rate correlates with the modulation depth getting higher the stronger the modulation depth is (see Fig. 4.12 A). However, it seems like the spike rate is equally rising for ICNs of Otof ${ }^{1515 T / I 515 T}$ mice and wildtype littermates up to a modulation depth of $100 \%$ where the mutants have only a mild increase in spike rates while the wildtypes show a strong increase (not significant, see Fig. 4.12 B). Adaptation is very strong at low modulation frequencies with a decay ratio of about 0.2 and gets weaker at higher modulation depths (see Fig. $4.12 \mathrm{C}$ ). This is true at least for the measured modulation frequency of $16 \mathrm{~Hz}$ where phase-locking is still high. The failure rate shows an inverse correlation with modulation depth, matching the increase in spike rate with an increase in modulation depth (see Fig. 4.12 D). The deeper the modulation, the less likely it is that a phase is skipped by a neuron and no spikes are elicited. Moreover, the vector strength shows a strong correlation with modulation depth as expected (see Fig. 4.12 E). The higher the modulation depth, the more precise the neurons can phase-lock to the amplitude modulation. Significant differences between wildtype and mutant ICNs could not be observed.

In summary, the ICC is capable of phase-locking to the envelope of sAM tones up to approximately $64-128 \mathrm{~Hz}$. In this range, the ICNs from Otof ${ }^{I 515 T / I 515 T}$ mice show similar abilities in phase-locking to the ICNs from $\mathrm{Otof}^{+/+}$littermates. However, adaptation is strongly increased in ICNs of Otof ${ }^{1515 T / I 515 T}$ mice in exactly that range where phaselocking is possible. In addition to that, spike rates are significantly reduced, and the 
failure rate is increased at intermediate modulation frequencies in Otof ${ }^{I 515 T / I 515 T}$ ICNs. The spike rate and failure rate correlate with the sound intensity and the modulation depth, but differences between wildtype and mutant ICNs could not be observed for these stimulations due to the low number of samples.
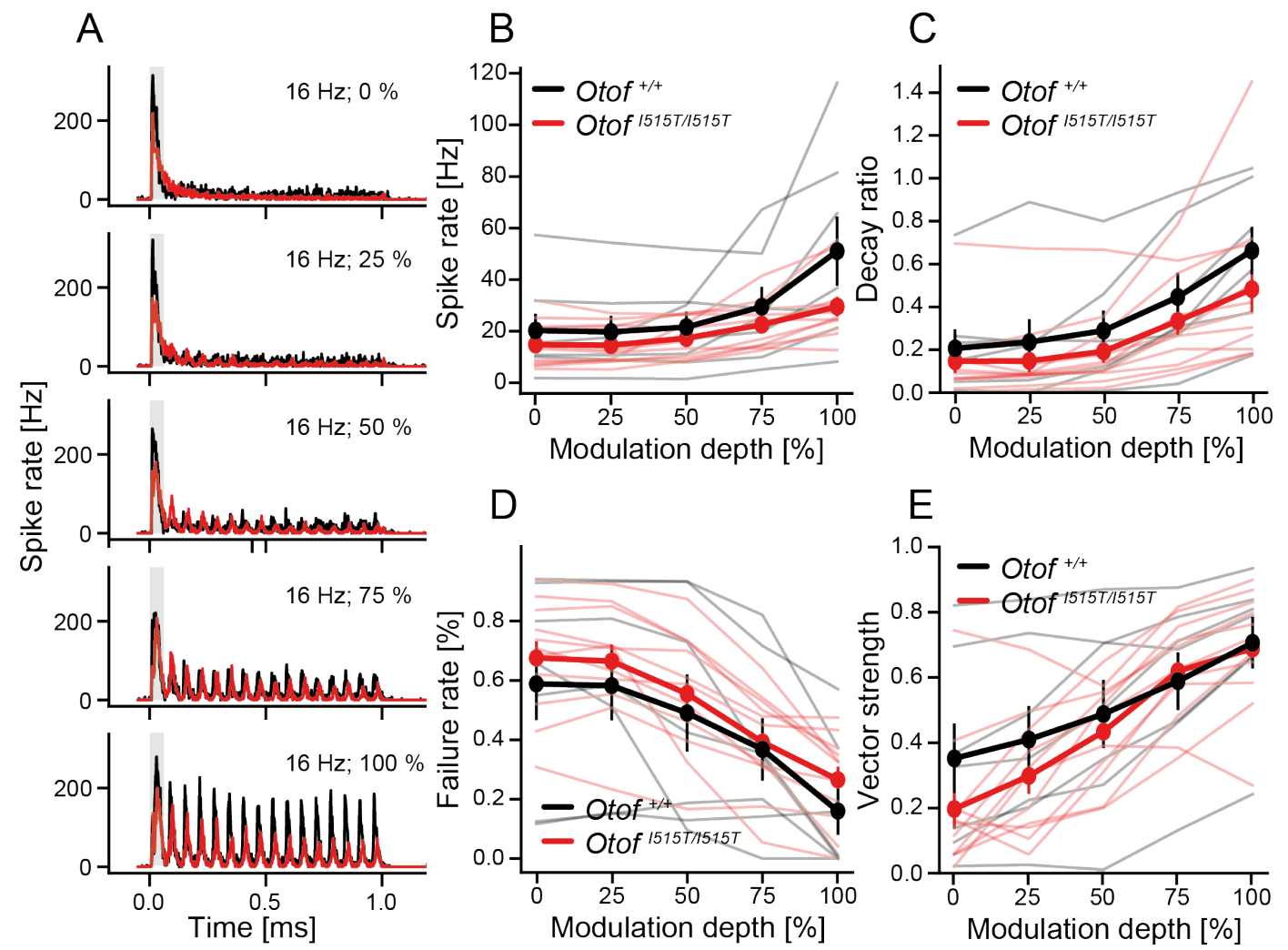

Figure 4.12.: Responses to amplitude modulated tones with varying modulation depths are similar between ICNs from Otof $^{1515 T / I 515 T}$ and Otof $^{+/+}$mice.

(A) Averaged PSTHs for each tested modulation depth at $16 \mathrm{~Hz}$ modulation frequency and dBOpt for ICNs of Otof ${ }^{1515 T / I 515 T}$ mice $(\mathrm{n}=11)$ and wildtype littermates $(\mathrm{n}=7)$. Duration of one cycle is marked in grey. (B) Rate modulation transfer function, which plots the averaged spike rate throughout the whole $1 \mathrm{~s}$ stimulation against the modulation frequency. Spike rates are not significantly different but might possibly be higher in ICNs of wildtype animals. (C) The decay ratio, expressing the adaptation over time during amplitude modulated tones, shows that the adaptation is stronger at low modulation depths than at high modulation depths, but does not differ significantly between wildtype and mutant ICNs. (D) The failure rate correlates with the modulation depth. The more precise the modulation is, the lower is the failure rate. (E) Synchronization modulation transfer function, which shows the vector strength as a measure of phase-locking plotted against modulation frequency shows a normal precision of spike timing, that is dependent on the modulation depth. (B-E) No significant differences could be seen in this stimulation paradigm, although there is a trend towards slightly impaired Otof ${ }^{I 515 T / I 515 T}$ ICNs. Circles in (B-D) represent mean \pm SEM. The full statistical analysis is shown in appendix B. 


\subsection{Otoferlin pachanga mice (Otof $\left.{ }^{P g a / P g a}\right)$}

The Otof ${ }^{P g a / P g a}$ mice have a mutation in the hair-cell specific protein otoferlin, just like the Otof ${ }^{I 515 T / I 515 T}$ mice. However, Otof ${ }^{P g a / P g a}$ mice are perceptional deaf and sound evoked vesicle exocytosis is almost completely abolished. Recording of single units has shown to be very difficult in the AN where very long silent intervals were needed to get sound evoked spiking. However, the IC is known to be able to compensate for reduces input spike rates by increasing central gain, so we were wondering how much the IC can compensate for the pachanga mutation.

In single unit recording from the ICC of Otof ${ }^{P g a / P g a}$ mice, sound evoked spiking was strongly impaired as well. Single units could only be found during stimulation with $90 \mathrm{~dB}$ SPL, instead of the formerly used $70 \mathrm{~dB}$ SPL. FRAs could be measured normally, as the randomization of stimuli lead to longer intervals between frequencies a single unit is responsive for. Nevertheless, the FRAs of all measured units were very broad and CFs were hard to determine. The FRAs of three representative units are shown in Fig. $4.13 \mathrm{~A}$.

Rate-level functions for each unit were measured with $100 \mathrm{~ms}$ tone bursts at a repetition rate of $1 \mathrm{~Hz}$. The rate-level function of a representative neuron, which corresponds to the FRA in the upper panel, is shown in Fig. 4.13 B. With a threshold of $85 \mathrm{~dB}$ SPL, considerable spiking was only elicited at $90 \mathrm{~dB}$ SPL.

To test, up to which rates this unit can still follow the stimuli we measured PSTHs with $1 \mathrm{~Hz}, 0.5 \mathrm{~Hz}$ and $0.25 \mathrm{~Hz}$ at $90 \mathrm{~dB}$ SPL (see Fig. $4.13 \mathrm{C}$ ). At $1 \mathrm{~Hz}$ stimulation rate (C1), only the first three repetitions elicited a reliable onset response. At $0.5 \mathrm{~Hz}$ stimulation rate $(\mathrm{C} 2)$ this unit elicited an onset spike at more than half of the repetitions and at $0.25 \mathrm{~Hz}$ stimulation rate (C3) every repetition of the tone elicited one onset spike.

Averaging all measured ICNs from Otof ${ }^{P g a / P g a}$ mice, we were accessing broadness of tuning by calculating the responsive range from the smoothed FRA at $90 \mathrm{~dB}$ SPL for each unit. As the Otof ${ }^{P g a / P g a}$ mice are a homozygous strain and no wildtype littermates exist we compared the values to the "V-shaped" units of wildtype mice with the same background $(\mathrm{C} 57 \mathrm{Bl} / 6)$. The ICNs from Otof Pga/Pga mice tended to have broader FRAs (see Fig. 4.14 A). Furthermore, all Otof ${ }^{P g a / P g a}$ ICNs had a pure tone threshold above $70 \mathrm{~dB}$ SPL and all of them increased their spike rate up to $90 \mathrm{~dB}$ SPL, the highest intensity tested (see Fig. 4.14B). In addition to that, none of the measured units responded with more than a single onset spike per presentation of a tone, apart from a low spontaneous spike rate. At $1 \mathrm{~Hz}$ stimulation rate most units barely responded to more than the first repetitions. At $0.5 \mathrm{~Hz}$ stimulation rate the averaged PSTH shows a considerable onset response which is half as big as the averaged onset rate in the $0.25 \mathrm{~Hz}$ stimulation condition (see Fig. 4.14 C).

For the pachanga mutation with a drastically reduced vesicle exocytosis at the IHC ribbon synapse, the ICC was not able to compensate this severe defect. Up to 4 seconds of recovery time were needed for a neuron to be able to reliably respond to a presented tone with only one onset spike. 
A

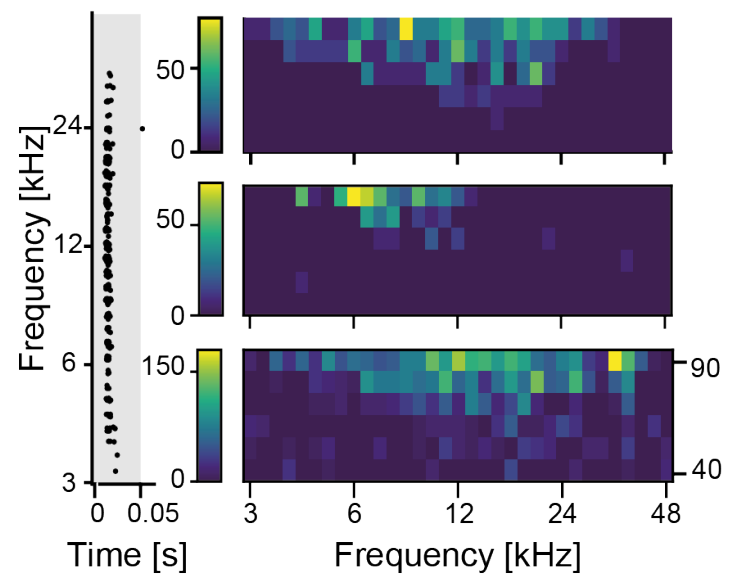

B

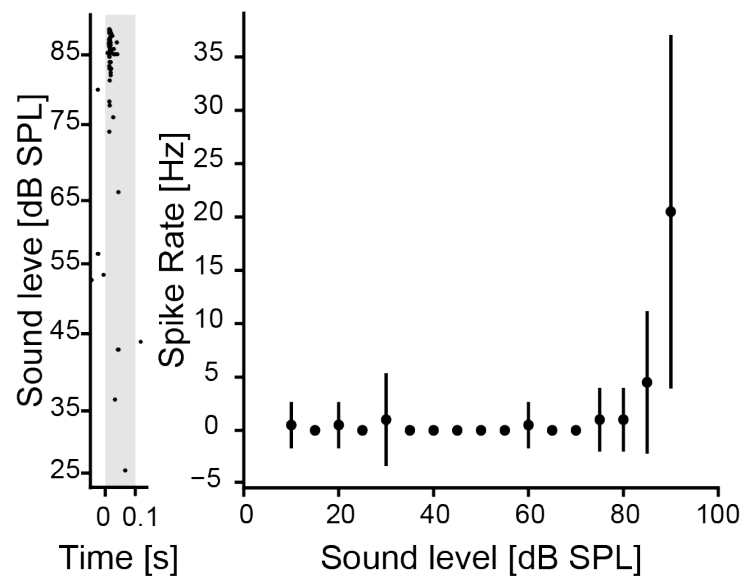

C1

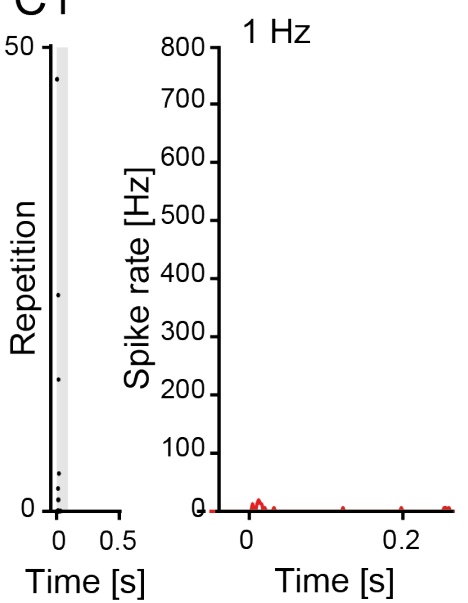

$\mathrm{C} 2$

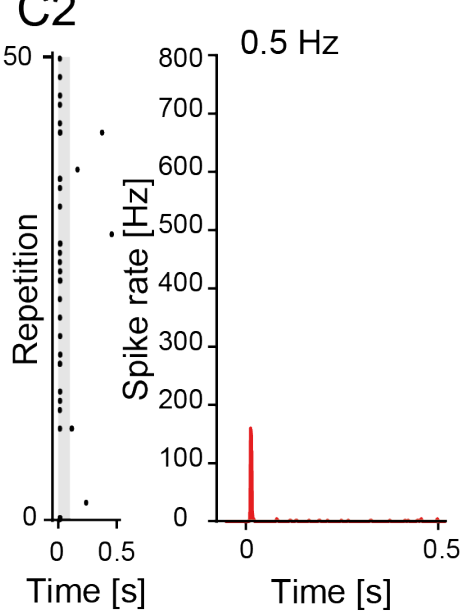

C3

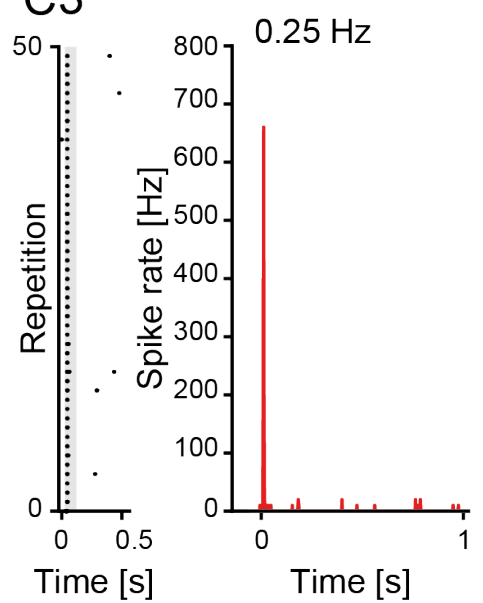

Figure 4.13.: Responses of a representative ICN from an Otof ${ }^{\text {Pga/Pga }}$ mouse.

(A) FRA examples of three different units. FRAs are broadened and a CF can hardly be determined. The scatter plot belongs to the upper FRA. In this example $11004 \mathrm{~Hz}$ was selected as CF. The following panels (B-C) show the same example unit as in the upper panel. (B) The threshold of this unit is very high and considerable responses can only be seen at $90 \mathrm{~dB}$ SPL, the highest measured intensity. (C) PSTH of the Otof ${ }^{P g a / P g a}$ unit in response to pure tones at CF and $90 \mathrm{~dB}$ SPL presented at $1 \mathrm{~Hz}$ (left), $0.5 \mathrm{~Hz}$ (middle) and $0.25 \mathrm{~Hz}$ (right). In all cases, if at all only one spike per presentation of the tone was detected. For the $1 \mathrm{~Hz}$ stimulation, the unit responded reliably only to the first three repetitions. At the stimulation rate of $0.5 \mathrm{~Hz}$ about half of the repetitions elicited a spike and at $0.25 \mathrm{~Hz}$ stimulation a spike was elicited in every repetition. In the scatter plots the time when the stimulus was presented is colored in grey. 

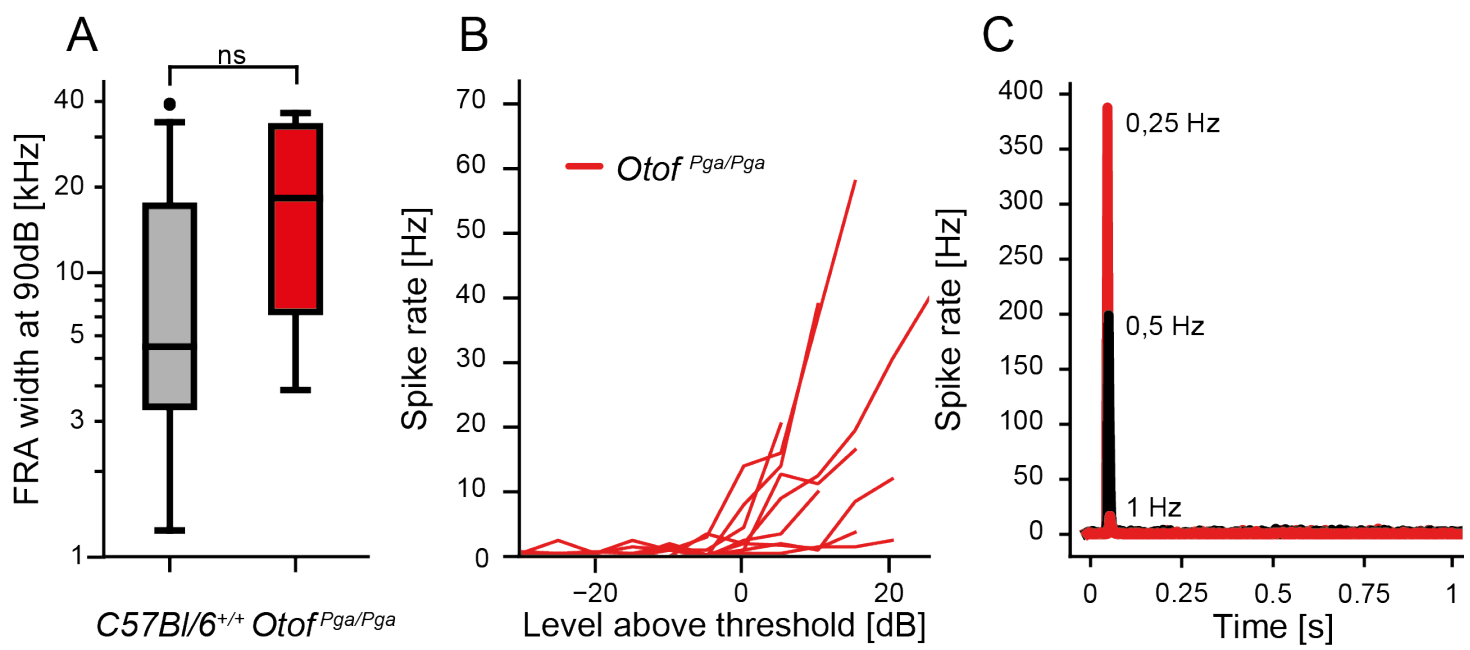

Figure 4.14.: Frequency tuning, rate-level functions and PSTHs from ICNs of Otof ${ }^{\text {Pga/Pga }}$ mice.

(A) The FRA width at $90 \mathrm{~dB}$ SPL showed a trend towards broader FRAs in ICNs of Otof Pga/Pga mice $(\mathrm{n}=8)$ compared to $\mathrm{C} 57 \mathrm{Bl} / 6$ wildtype mice $(\mathrm{n}=23$, Mann-Whitney-U-test $\mathrm{p}=0.1)$. (B) All measured rate-level functions are plotted relative to their threshold $(\mathrm{n}=9)$. No rate-level function had a threshold lower than $70 \mathrm{~dB}$ SPL and all fibers had monotonic properties with the highest rate at $90 \mathrm{~dB}$ SPL, the highest measured intensity. (C) Overlay of the averaged PSTH from 3 stimulation frequencies. Only onset spikes were elicited in all units at all stimulation frequencies. For $1 \mathrm{~Hz}$ stimulation barely any spikes were detected $(\mathrm{n}=9)$. At $0.5 \mathrm{~Hz}$ stimulation a reasonable onset response can be seen $(\mathrm{n}=8)$, which is doubling in spike rate when halving the stimulation frequency to $0.25 \mathrm{~Hz}(\mathrm{n}=5)$. 


\subsection{Quiverer-3J mice $\left(S p n b^{q v-3 J / q v-3 J}\right)$}

Spectrin is a protein localized in the AIS and nodes of Ranvier of all neurons where it's important for clustering sodium channels. Mutations in spectrin cause the "quiverer"phenotype in mice, which results in sensory- and motor neuropathies, accompanied by deafness or hearing impairment, due to a reduced velocity of action potential initiation and propagation.

In contrast to the Otof ${ }^{I 515 T / I 515 T}$ mutation, the $S p n b^{q v-3 J / q v-3 J}$ mutation is not located in the IHCs. The first affected stage of the auditory pathway is the SGNs, which directly contact the IHCs and the mutation is present in all following neurons of the auditory system.

We are now investigating the effect of delayed inputs into the ICNs on sound encoding in the ICC of $S p n b^{q v-3 J / q v-3 J}$ mice. As the action potentials have to travel a longer distance to the recording site in the ICC than in the SGNs, we expect a noticeable delay of spiking in the ICNs of $S p n b^{q v-3 J / q v-3 J}$ mice.

In vivo single unit recordings from the ICC were performed on $16 S p n b^{q v-3 J / q v-3 J}$ mice and $14 S p n b^{+/+}$littermates. The same stimulus protocol was run as for Otof ${ }^{I 515 T / I 515 T}$ mice and similar analysis were performed, including general organization in the ICC, spike rates and spike timing, responses to sAM tones and forward masking. Additionally, we included a tone burst stimulation with $50 \mathrm{~ms}$ tone bursts presented at $5 \mathrm{~Hz}$ stimulation frequency.

\subsubsection{Spontaneous rate is reduced while tuning and thresholds are normal}

Firstly, we again wanted to find out whether neuronal architecture in the ICC is affected by the $S p n b^{q v-3 J / q v-3 J}$ mutation, concerning distribution of spontaneous rates, CFs or thresholds.

In the ICC, spontaneous rate is significantly reduced similar to the spontaneous rate in SGNs, despite being generally very low (see Fig. 4.15 A). Tonotopy in the ICC is intact, as the distribution of CFs is not different between ICNs from $S p n b^{q v-3 J / q v-3 J}$ mice compared to wildtype littermates and correlates with unit depth (see Fig. 4.15 B). Interestingly, although there is a clear correlation between unit depth and $\mathrm{CF}$, confirming that the tonotopy is intact, and the recording site was the ICC, no mutant ICNs were found below $1300 \mu \mathrm{m}$. This is probably due to the fact, that $S p n b^{q v-3 J / q v-3 J}$ animals are generally smaller than their $S p n b^{+/+}$littermates. Distribution of thresholds and of dBOpt of all measured ICNs is normal in Spnb ${ }^{q v-3 J / q v-3 J}$ mice (see Fig. 4.15 C-D). 
A

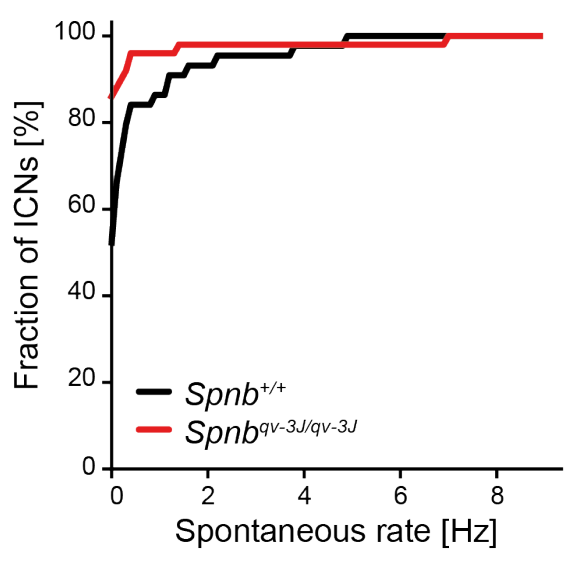

C

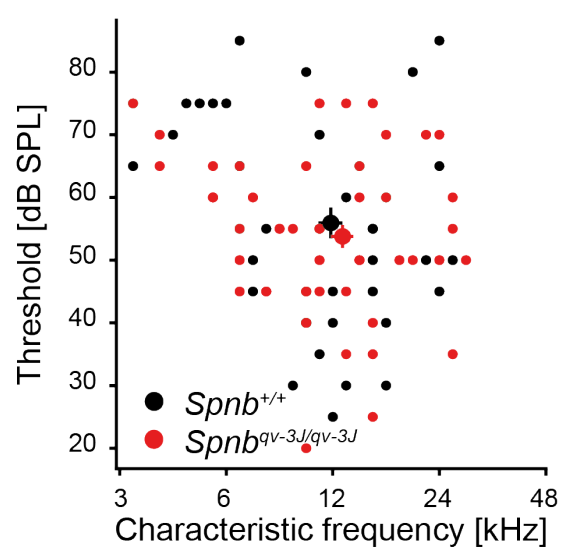

B

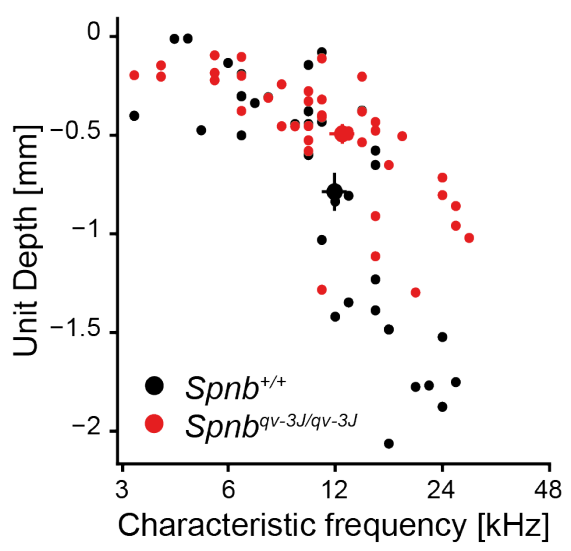

D

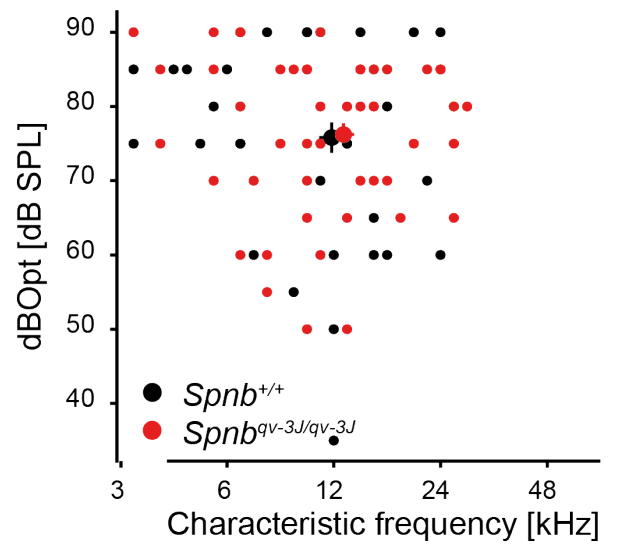

Figure 4.15.: Spontaneous rate is reduced while tuning and thresholds are normal in ICNs of $S p n b^{q v-3 J / q v-3 J}$ mice.

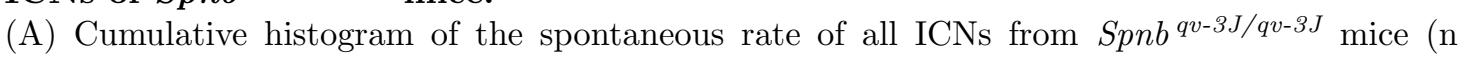
$=50)$ and wildtype littermates $(\mathrm{n}=44)$. Spontaneous rate is significantly lower for ICNs of Spnb ${ }^{q v-3 J / q v-3 J}$ mice (Kolmogorov-Smirnov-test, $\mathrm{p}=0.0002$ ). (B) Depth of the recording site in the brain as a function of CF. Distribution of CFs is normal and correlates with the unit depth. However, no single units could be found below $1300 \mu \mathrm{m}$ for $\operatorname{Spnb} b^{q v-3 J / q v-3 J}$ mice $(\mathrm{n}=40)$ in comparison to wildtype littermates $(\mathrm{n}=38)$, which is likely due to their generally smaller size. (C) Threshold of each unit plotted against CF. Thresholds were normal in ICNs of Spnb ${ }^{q v-3 J / q v-3 J}$ mice $(\mathrm{n}=43)$ compared to wildtype littermates $(\mathrm{n}=49)$. (D) dBOpt for each unit plotted against CF. Distribution of dBOpt was normal in ICNs of $S p n b^{q v-3 J / q v-3 J}$ mice $(\mathrm{n}=49)$ compared to wildtype littermates $(\mathrm{n}=43)$.

(B-D) Big circles represent mean values \pm SEM. The full statistical analysis is shown in appendix B.

\subsubsection{Intensity coding is unaffected in $S p n b^{q v-3 J / q v-3 J}$ mice}

Pure tone thresholds in SGNs, as well as in cells from the AVCN and from the ICC were normal and only ABR thresholds were slightly elevated. Therefore, impairments in intensity coding are not expected for ICNs of Spnb ${ }^{q v-3 J / q v-3 J}$ mice. 
Comparing the shape of rate-level functions between wildtype and mutant mice, we don't see any anomalies for monotonic fibers (see Fig. 4.16 A). For non-monotonic fibers we see a few fibers with a very steep rise in spiking at the threshold (see Fig. 4.16 D), but the mean and maximal spike rates are not significantly different for neither the group of monotonic fibers (see Fig. 4.16 B), nor the group of non-monotonic fibers (see Fig. 4.16 E). Furthermore, we calculated the dynamic range for monotonic fibers by fitting a sigmoid function and of non-monotonic fibers by subtracting the threshold from dBOpt. No significant difference in dynamic range between ICNs of Otof ${ }^{I 515 T / I 515 T}$ mice compared to wildtype littermates were found for monotonic (see Fig. $4.16 \mathrm{C}$ ) and non-monotonic fibers (see Fig. $4.16 \mathrm{~F}$ ).

As expected, the $S p n b^{q v-3 J / q v-3 J}$ mutation does not cause any impairment in coding of different sound intensities.
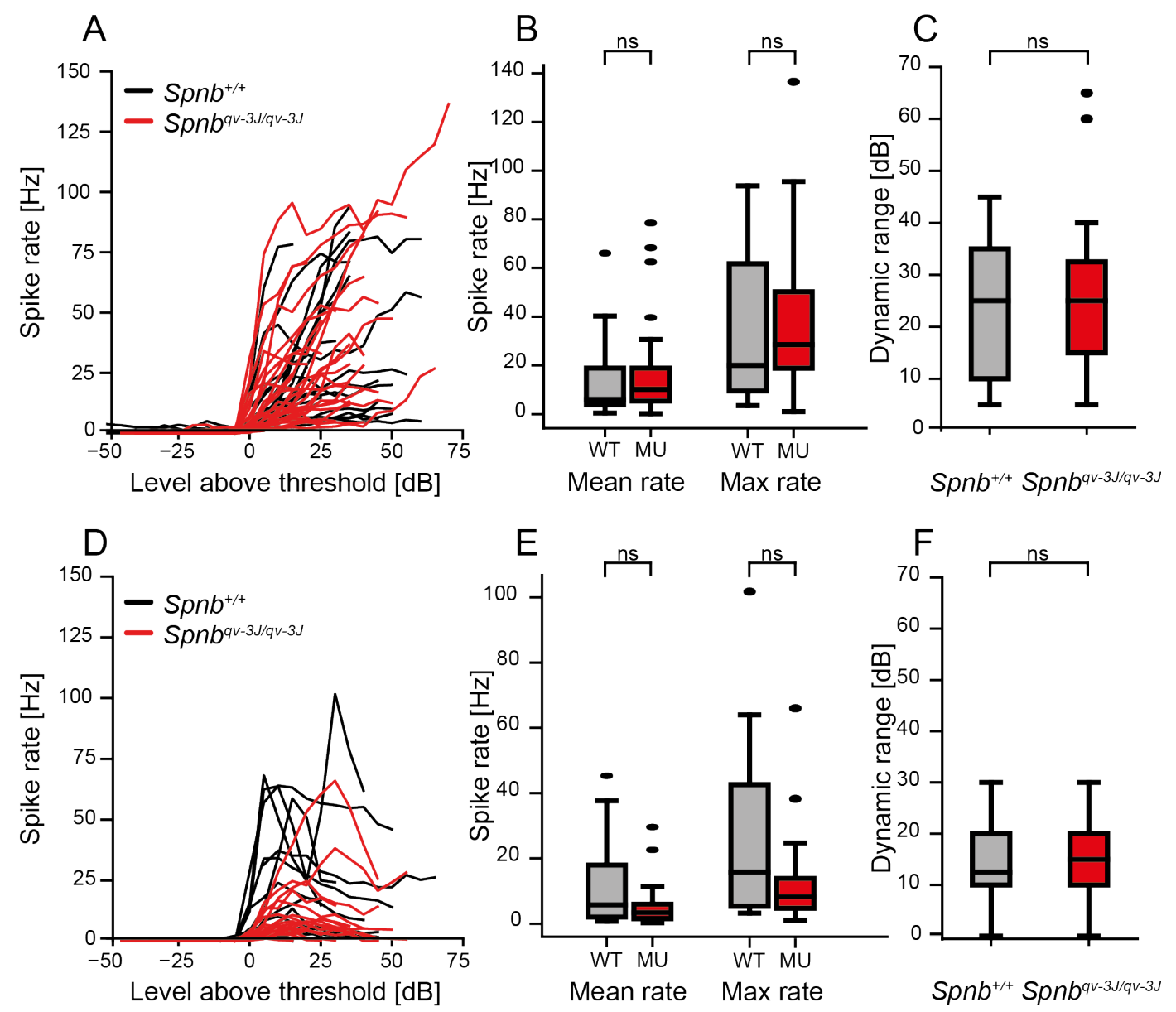

Figure 4.16.: Intensity coding is normal in $S p n b^{q v-3 J / q v-3 J}$ mice.

Rate-level functions of all units with a monotonicity index (A) higher than 0.75 and (D) lower than 0.75 plotted relative to their threshold. Mean and maximal spike rates did not differ between (B) wildtype $(\mathrm{n}=27)$ and mutant mice $(\mathrm{n}=31)$ for monotonic units and $(\mathrm{E})$ wildtype $(\mathrm{n}=16)$ and mutant mice $(\mathrm{n}=19)$ for non-monotonic units. Dynamic range was comparable between wildtype and mutants for both (C) monotonic and (F) non-monotonic single units. The full statistical analysis is shown in appendix B. 


\subsubsection{Coding of short tones might be affected}

When analyzing all measured ICNs and comparing the distribution of minimal tone length needed to elicit a response, ICNs of $S p n b^{q v-3 J / q v-3 J}$ mice have a significantly increased averaged minimal tone length compared to $S p n b^{+/+}$littermates, indicating a deficit in the coding of short tones (see Fig. 4.17 A).

In contrast to the Otof $f^{I 515 T / I 515 T}$ mice the $S p n b^{q v-3 J / q v-3 J}$ mice have a different response pattern between "onset"- and "sustained"-type neurons. "Onset" neurons were almost entirely able to detect even the shortest tone length of $8 \mathrm{~ms}$ while "sustained" neurons had a broader distribution and minimal tone length ranged between 8-48 ms for wildtypes. Looking at both subgroups individually, we could not find significant differences between ICNs of $S p n b^{q v-3 J / q v-3 J}$ mice and $S p n b^{+/+}$littermates (see Fig. 4.17 B-C).
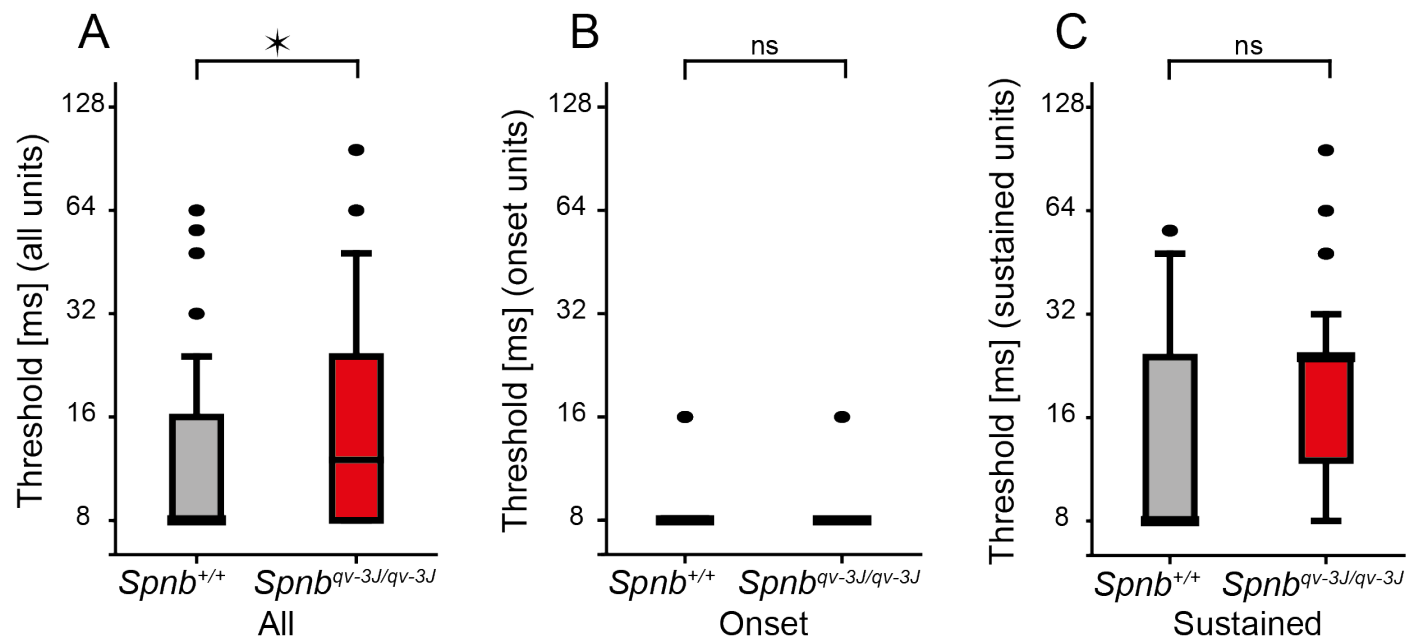

Figure 4.17.: Coding of short tones might be affected in ICNs of $S_{p n b^{q v-3 J / q v-3 J}}$ mice. (A) The minimal tone length needed to elicit a response between all units from $S p n b^{q v-3 J / q v-3 J}$ mice $(\mathrm{n}=31)$ compared to wildtype littermates $(\mathrm{n}=32)$ was significantly higher (Mann-WhitneyU-test $\mathrm{p}=0.045)$, indicating a defect in coding of short tones. (B, C) The $S p n b^{q v-3 J / q v-3 J}$ mice show a clear distinction in response patterns between "onset"-type (wildtype $\mathrm{n}=13$, mutant $\mathrm{n}=8$ ) and "sustained"-type (wildtype $\mathrm{n}=13$, mutant $\mathrm{n}=21$ ) neurons. (B) Units with an "onset"-type response were able to respond to all tone lengths. (C) Units with an "sustained" response pattern needed longer minimal tone lengths and were not in all cases able to respond to very short tones. Both subgroups do not show any significant difference between wildtype and mutant ICNs. The full statistical analysis is shown in appendix B.

\subsubsection{Onset responses have a use dependent delay of spiking and increased jitter}

Pure tone responses, in particular spike latency is very interesting when investigating the effect of the $S p n b^{q v-3 J / q v-3 J}$ mutation on the sound encoding in the ICC.

Firstly, we wanted to compare the peak and steady-state spike rates. To visualize these, we created a peak-aligned PSTH, where all onset responses from all ICNs are aligned on 
the time scale (see Fig. 4.18 A). Quantification of average peak rates were significantly reduced for ICNs of $S p n b^{q v-3 J / q v-3 J}$ mice compared to wildtype littermates, comparable to the SGN responses (see Fig. 4.18 B). For the quantification of steady-state rates, "onset"type neurons, which do not possess a steady-state response, were excluded. Still, the steady-state rate did not differ between wildtype and mutant ICNs.

Next, we were interested in spike timing of ICNs from $S p n b^{q v-3 J / q v-3 J}$ mice. As the $S p n b^{q v-3 J / q v-3 J}$ mutation, according to the ABRs, causes an increasing delay, the longer the way the signals have to travel, we were expecting an increase in FSL. To visualize this, we plotted a normal PSTH without peak alignment in Fig. 4.18 D. Here, the reduction in onset rate can be observed, as well as a slight shift of the whole response in time. To quantify this time shift, we calculated the median FSL of all ICNs. This was strongly increased from a median of $16 \mathrm{~ms}$ for wildtypes to a median of $25 \mathrm{~ms}$ for $S p n b^{q v-3 J / q v-3 J}$ mutants (see Fig. 4.18 E). The variance of FSL did not show greater jitter between genotypes (see Fig. 4.18 F).

Additionally, we were trying to fit a single- or double-exponential function to the PSTH time course to calculate time constants for adaptation. However, the heterogeneity of responses from single neurons was too high to return convincing fits and time constants.

In addition to the PSTH analysis shown before, measured with $200 \mathrm{~ms}$ tone burst presented at $1.5 \mathrm{~Hz}$ stimulation frequency, we implemented a fast-stimulating PSTH, measured with $50 \mathrm{~ms}$ tone bursts presented at $5 \mathrm{~Hz}$ stimulation frequency for the analysis of Spnb ${ }^{q v-3 J / q v-3 J}$ mice. Here, again we generated a peak-aligned PSTH so visualize spike rates (see Fig. 4.19 A). The onset and adapted rates seem to be generally less precise in the fast stimulation condition and are harder to identify. Nevertheless, the quantification of peak rate leads to the same amount of rate reduction as in the slow stimulation condition (see Fig. 4.19 B). The steady-state rate was calculated the same way as before, excluding all "onset" type units and was not significantly different in the fast stimulation condition between wildtype and mutant ICNs. The spike rate itself and the reduction of peak rate in $S p n b^{q v-3 J / q v-3 J}$ ICNs seems to be independent of the stimulation frequency, although the shape of the PSTH is slightly distorted.

In the normal PSTH without peak-alignment (see Fig. 4.19D) the increase in FSL can be seen very well by a shift of the whole response. Moreover, the peak and steady-state rates are barely distinguishable.

The calculated difference in median FSL between ICNs from Spnb ${ }^{q v-3 J / q v-3 J}$ mice and $S p n b^{+/+}$littermates was strongly delayed and more than doubled from $15 \mathrm{~ms}$ to $33 \mathrm{~ms}$ (see Fig. $4.19 \mathrm{E}$ ). This $18 \mathrm{~ms}$ increase of FSL is even double the amount of that in the slow stimulation condition. Furthermore, the variance of FSL is strongly increased in ICNs of $S p n b^{q v-3 J / q v-3 J}$ mice in the fast stimulation condition (see Fig. $4.19 \mathrm{~F}$ ), while the variance of FSL is similar in wildtype ICNs for both conditions. This leads to the conclusion, that when stimulating with a higher frequency, wildtype neurons can still respond normally while ICNs from Spnb ${ }^{q v-3 J / q v-3 J}$ mice react to the higher stimulation frequency with a further delay of responses and an increased jitter. While responding comparably normal at low stimulation frequencies, they show a strong dependence on stimulus rates. 

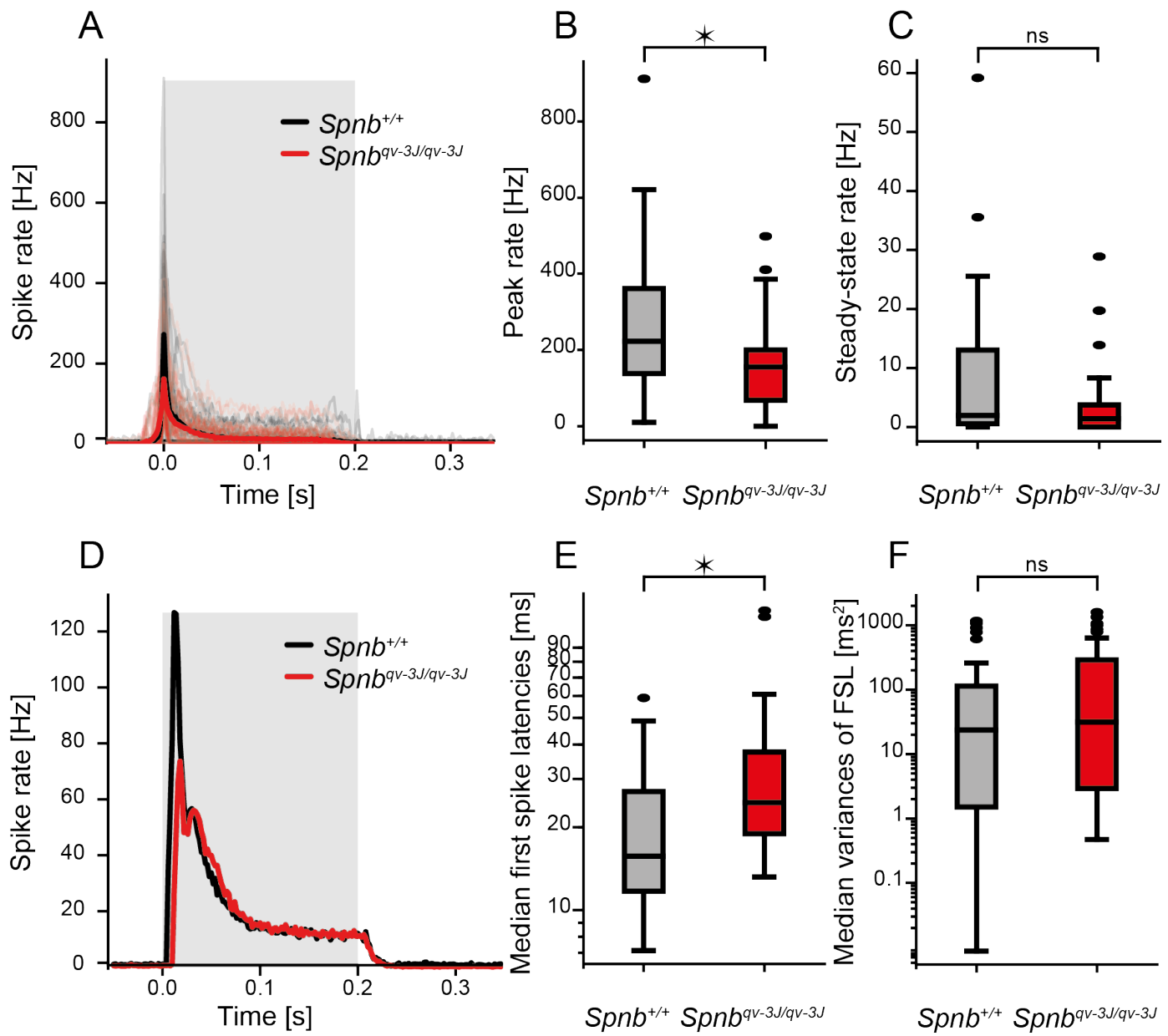

Figure 4.18.: Onset responses are reduced and delayed in ICNs of $S p n b^{q v-3 J / q v-3 J}$ mice.

(A) Averaged peak-aligned PSTH of wildtype $(\mathrm{n}=38)$ and mutant $(\mathrm{n}=45)$ ICNs. The time when the stimulus was presented is colored in grey. (B) Quantification of the peak rate. The onset rate of ICNs from $S p n b^{q v-3 J / q v-3 J}$ mice is significantly lower than that of wildtype littermates (Kruskal-Wallis-test, $\mathrm{p}=0.003$ ). $(\mathrm{C})$ For quantification of the steady-state rate all "onset"-type neurons, which do not have any steady-state response, were excluded. The steady-state rate did not differ between ICNs of Spnb $b^{q v-3 J / q v-3 J}$ mice $(\mathrm{n}=30)$ compared to wildtype littermates $(\mathrm{n}=$ 21). (D) Averaged PSTH of wildtype and mutant ICNs without peak alignment show a slight shift in timing. The time when the stimulus was presented is colored in grey. (E) Median FSL was strongly increased in ICNs of $S p n b^{q v-3 J / q v-3 J}$ mice from a median of $16 \mathrm{~ms}$ in wildtype to $25 \mathrm{~ms}$ in mutant ICNs. (F) Variance of FSL did not show greater jitter between genotypes. The full statistical analysis is shown in appendix B. 

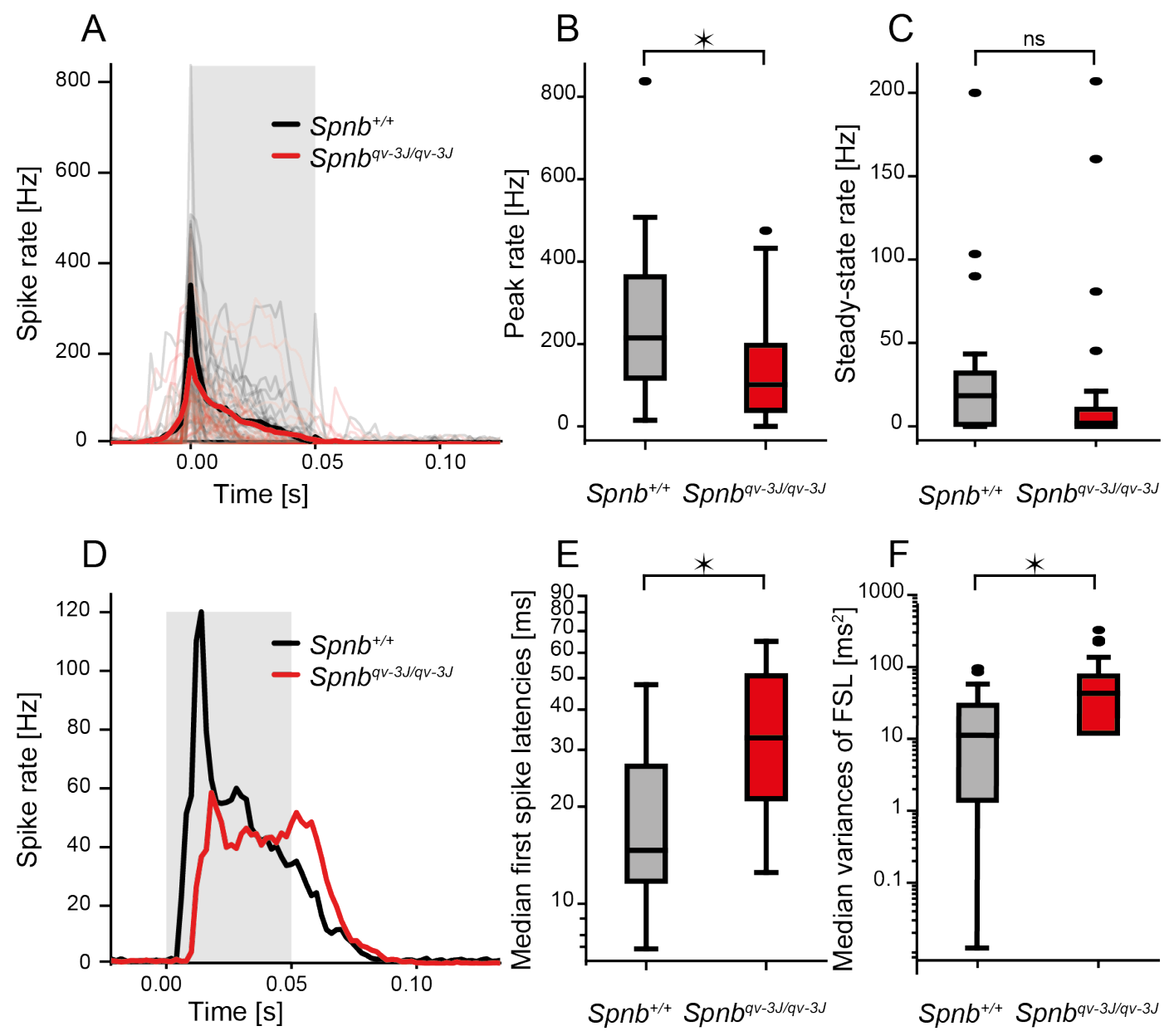

Figure 4.19.: Use dependent increase in delay of spiking and in variance of first-spike latency in ICNs of $S p n b^{q v-3 J / q v-3 J}$ mice.

(A) Averaged peak-aligned PSTH of wildtype $(\mathrm{n}=36)$ and mutant $(\mathrm{n}=34)$ ICNs. Onset and steady-state response are less pronounced at higher stimulation frequencies. The time when the stimulus was presented is colored in grey. (B) Quantification of the peak rate shows a significantly lower rate for ICNs of Spnb ${ }^{q v-3 J / q v-3 J}$ mice (Mann-Whitney-U-test, $\mathrm{p}=0.001$ ). (C) For quantification of the steady-state rate all "onset"-type neurons, which do not have any steady-state response, were excluded. The steady-state rate does not differ between ICNs of Spnb ${ }^{q v-3 J / q v-3 J}$ mice $(\mathrm{n}=28)$ and wildtype littermates $(\mathrm{n}=23)$ at the fast stimulation. (D) Averaged PSTH of wildtype and mutant ICNs without peak alignment show a strong shift in spike timing. The onset response is barely distinguishable from the steady-state response for Spnb ${ }^{q v-3 J / q v-3 J}$ ICNs. The time when the stimulus was presented is colored in grey. (E) The median FSL is strongly increased in the fast stimulation condition from a median of $15 \mathrm{~ms}$ to $33 \mathrm{~ms}$ (Kruskal-Wallis-test, p <0.001.) (F) FSL was strongly more jittered for ICNs of Spnb ${ }^{q v-3 J / q v-3 J}$ mice in the fast stimulation condition (Kruskal-Wallis test, $\mathrm{p}=0.003$ ). The full statistical analysis is shown in appendix B. 


\subsubsection{Forward masking is unaffected in $S p n b^{q v-3 J / q v-3 J}$ mice}

As gap detection and replenishment were important features, which could not be compensated by the ICC for the Otof ${ }^{I 515 T / I 515 T}$ mutation, we were interested if there would be any effects from the $S p n b^{q v-3 J / q v-3 J}$ mutation on these features as well.

Similar to the normal forward masking in SGNs, depression of spiking was not different between ICNs of $S p n b^{q v-3 J / q v-3 J}$ mice compared to $S p n b^{+/+}$littermates (see Fig. 4.20 A). Furthermore, the minimal silent interval needed to elicit a probe response was similar for both genotypes (see Fig. 4.20 B).

These finding indicate that a delay in spike timing does not influence recovery from forward masking, no matter how long the delay is. Spike rates and minimal silent intervals are similar for both genotypes and depression of spiking does not increase due to a delayed response.
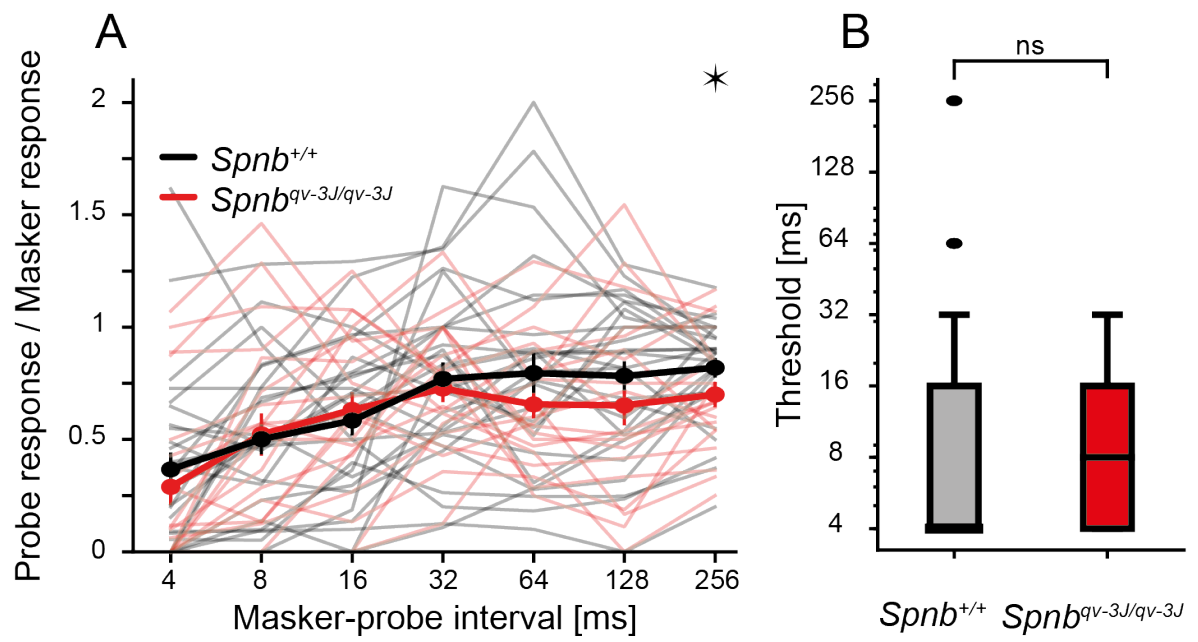

Figure 4.20.: Depression of spiking in a forward masking paradigm is unaffected in ICNs of $S p n b^{q v-3 J / q v-3 J}$ mice.

(A) Depression of spiking, measured by a ratio between probe and masker responses, is comparable between ICNs of Spnb ${ }^{q v-3 J / q v-3 J}$ mice $(\mathrm{n}=20)$ compared to wildtype littermates $(\mathrm{n}=28)$. However, note that the difference at $256 \mathrm{~ms}$ gap length is significant. Circles represent mean \pm SEM, asterisk represent significant differences. (B) The minimal silent interval needed to elicit a probe response is similar in both genotypes. The full statistical analysis is shown in appendix B.

\subsubsection{ICNs of $S p n b^{q v-3 J / q v-3 J}$ mice have a reduced maximal modulation frequency where phase-locking to sAM tones is possible}

One of the most interesting and also most challenging parts to analyze were the responses to sAM tones in the ICC. Interesting questions we addressed are how a delay in spike timing would affect the phase-locking to the envelope of sAM tones and if the whole response pattern would be merely shifted in time or be completely distorted. 
At first, we calculated the phase and vector strength and generated a circle diagram for each modulation frequency (see Fig. 4.21).

The shift in spike timing between ICNs of $S p n b^{q v-3 J / q v-3 J}$ mice and wildtype littermates can be seen by the difference in orientation of the two resulting vectors, which get more and more apart the higher the modulation frequency and consequently the shorter the phases get. In addition to that, the length of the vector representing the mutant ICNs seem to get shorter earlier than the one representing the wildtype ICNs.

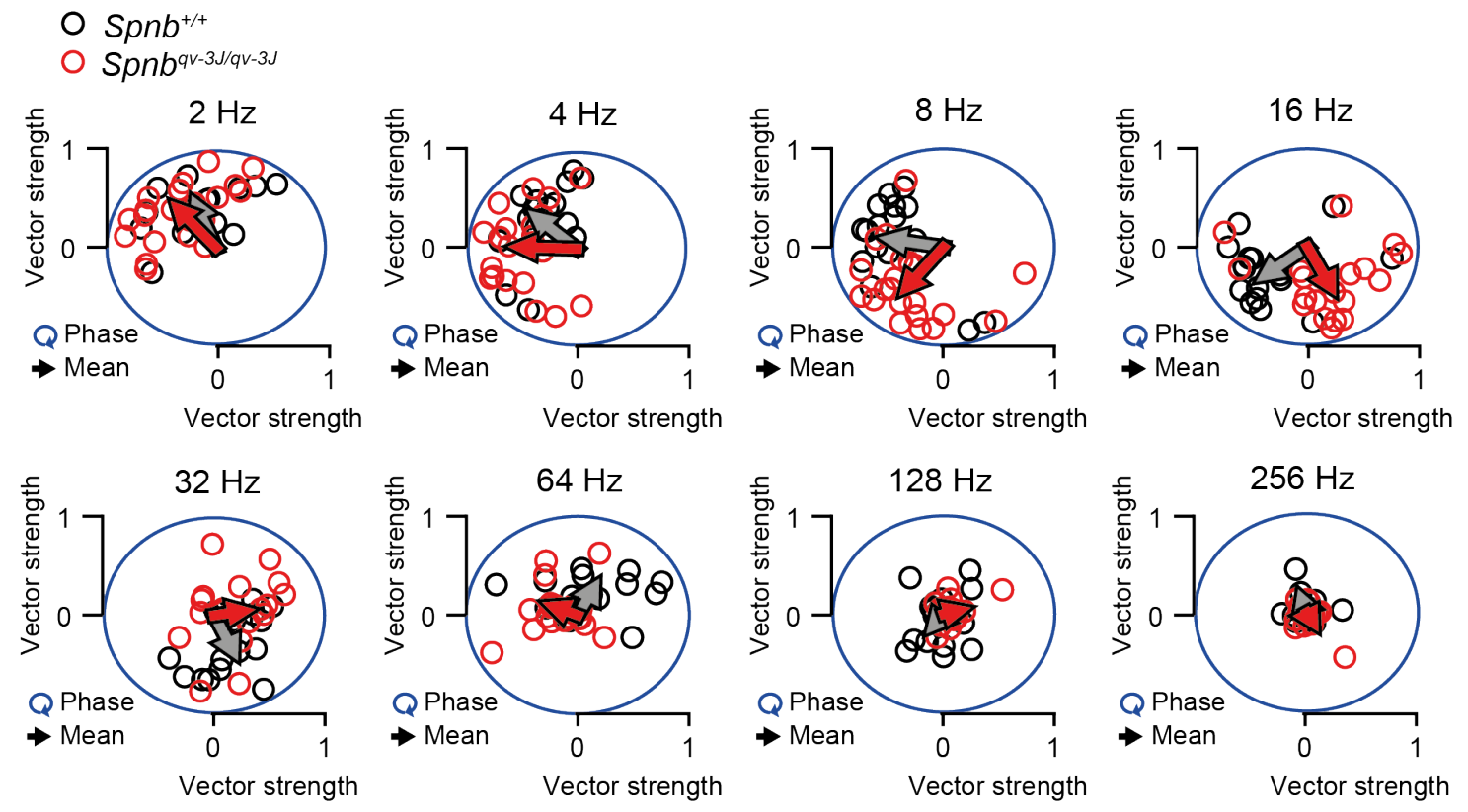

Figure 4.21.: Circle diagrams to depict phase-locking to amplitude modulated tones in ICNs from $S p n b^{q v-3 J / q v-3 J}$ mice.

Circle diagrams of vector strength and phase plotted for each modulation frequency. The phase corresponds to the position on the circumference while the vector strengths corresponds to the position on the radius. The farther outside, the higher is the vector strength. The corresponding vectors from all wildtype and mutant single units show similar length up to $16 \mathrm{~Hz}$. At higher frequencies the vectors representing the mutant response get shorter than those of wildtype responses. The delay of spiking can be seen by a shift in phase, represented by the discrepancy in orientation of the two vectors, which get more apart the shorter the phases get.

The delay of spike timing can be seen as well in the PSTHs for each modulation frequency in Fig. 4.22 A. Especially for low modulation frequencies a shift can be seen for every response peak in every phase, while at higher modulation frequencies a shift in the onset response is clearly visible.

Absolute spike rates throughout the whole stimulation for each modulation frequency are shown in Fig. 4.22 B and are only significantly reduced between $4-8 \mathrm{~Hz}$, in a range, where every phase might be perceived as a single onset response and as we showed before, onset responses were significantly reduced for pure tones. 
A

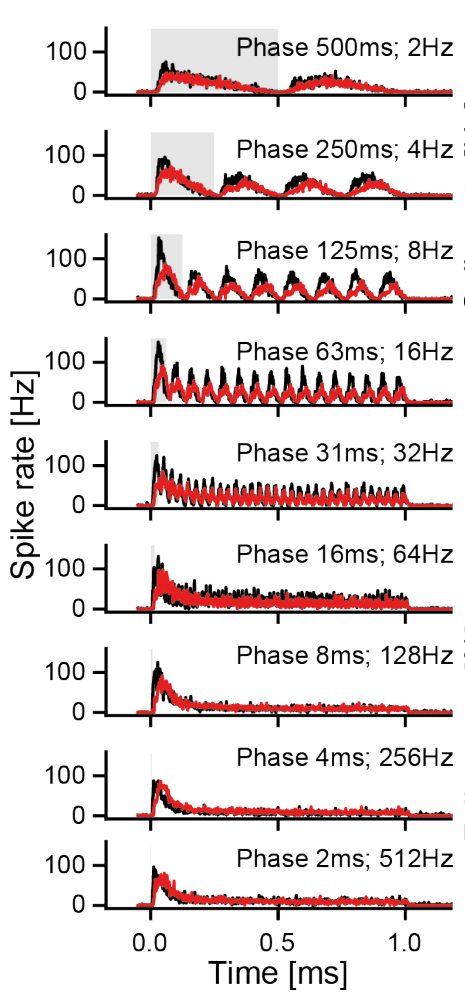

B

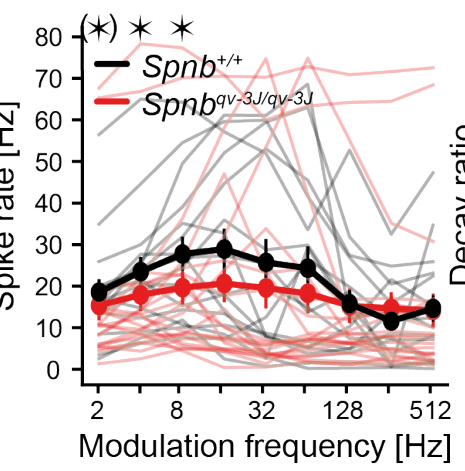

$\mathrm{D}$

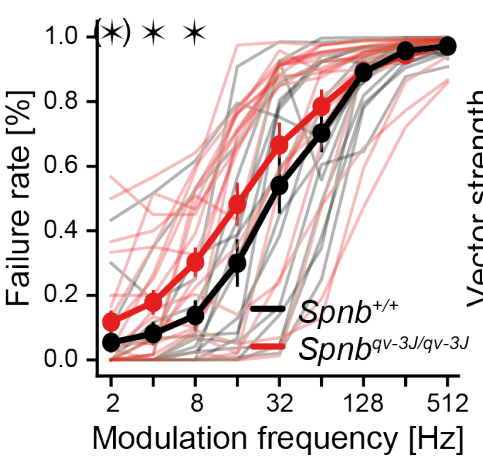

C

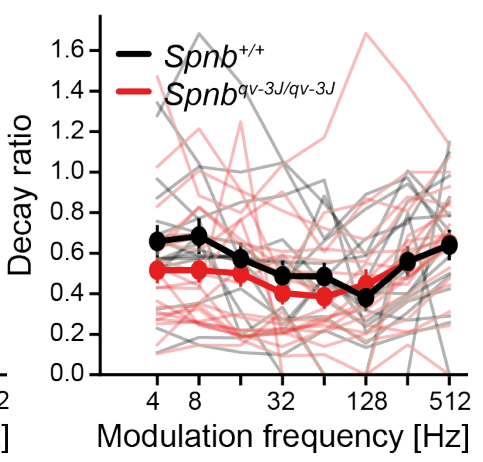

E

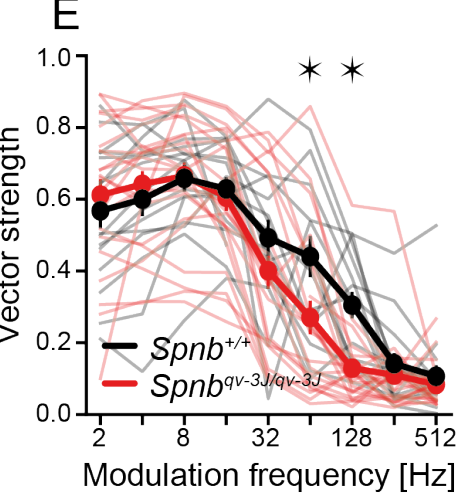

Figure 4.22.: Responses to amplitude modulated tones with varying modulation frequencies reveal only mildly reduced spike rates, but a defect in phase-locking at 64-128 Hz for ICNs of $S p n b^{q v-3 J / q v-3 J}$ mice.

(A) Averaged PSTHs for each modulation frequency separately from 2-512 Hz. The shift in spike timing between ICNs of $S p n b^{q v-3 J / q v-3 J}$ mice $(\mathrm{n}=22)$ and wildtype littermates $(\mathrm{n}=16)$ can be seen. Duration of one cycle is marked in grey. (B) Rate modulation transfer function, which plots the averaged spike rate throughout the whole $1 \mathrm{~s}$ stimulation against the modulation frequency. Spike rates are lower for slow stimulation at $4-8 \mathrm{~Hz}$ modulation frequency. (C) The decay ratio, expressing the adaptation over time during sAM tones, was not different between genotypes. (D) Failure rate plotted against modulation frequency. Comparable to the reduced spike rate, the failure rate was increased for low modulation frequencies between $4-8 \mathrm{~Hz}$. (E) Synchronization modulation transfer function, which shows the vector strength as a measure of phase-locking plotted against modulation frequency shows a significantly reduced vector strength at 32 and $64 \mathrm{~Hz}$ (Mann-Whitney-U test, $\mathrm{p}=0,029$ and $\mathrm{p}=0,001$ ). Precision of spike timing seems to be affected in a way, that phase-locking can only be kept at low modulation frequencies and drops even earlier than for wildtype units.

Circles in (B-D) represent mean \pm SEM. Asterisk represent significant differences, while asterisk in brackets represent no significance, but a difference which is still highly probable $(\mathrm{p}<0.07)$. The full statistical analysis is shown in appendix B.

Adaptation seems to be completely unaffected by the $S p n b^{q v-3 J / q v-3 J}$ mutation, despite delayed spike timing (see Fig. $4.22 \mathrm{C}$ ). However, the failure rate is significantly increased between $4-8 \mathrm{~Hz}$ modulation frequency (see Fig. 4.22 D), mirroring the reduction in spike rate. 
To investigate the ability to phase-lock to the envelope of sAM tones in more detail, we plotted the vector strength calculated for each ICN against its corresponding modulation frequency (see Fig. 4.22 E). Interestingly, the vector strength is still high at low modulation frequencies but decreases already for lower modulation in ICNs of $S p n b^{q v-3 J / q v-3 J}$ mice compared to Spnb ${ }^{+/+}$littermates. At $32 \mathrm{~Hz}$ modulation frequency the phaselocking abilities already get worse and at $128 \mathrm{~Hz}$ phase-locking is completely abolished. This difference between vector strength of wildtype and mutant ICNs is most prominent between $64-128 \mathrm{~Hz}$.

To further investigate the influence of the $S p n b^{q v-3 J / q v-3 J}$ mutation on the coding of sAM tones in the ICC, we recorded the responses to sAM tones with $16 \mathrm{~Hz}$ modulation frequency and varying intensities from 30-90 dB SPL as well as the responses to sAM tones with $16 \mathrm{~Hz}$ modulation frequency and varying modulation depths from 0-100 \% at dBOpt.

The PSTHs plotted for each measured sound intensity show an increase in spike rate with sound intensity for both genotypes (see Fig. 4.23 A). However, it seems as the spike rate of the ICNs from Spnb ${ }^{q v-3 J / q v-3 J}$ mice is slightly reduced compared to that of wildtype littermates. By plotting the absolute spike rates against the measured sound levels, we can indeed see a significant reduction at 30, 40 and $80 \mathrm{~dB}$ SPL, and besides, the average spike rate curve of mutant ICNs is below the wildtype average for all levels (see Fig. 4.23 B). This is in line with the $16 \mathrm{~Hz}$ stimulation condition in Fig. 4.22 B, which did not have significant difference between wildtype and mutant ICNs, although it seemed like it could be slightly reduced, as shown for 4 and $8 \mathrm{~Hz}$ modulation frequency.

Surprisingly, the decay ratio is decreased among all sound levels between 40-90 dB SPL (see Fig. $4.23 \mathrm{C}$ ), although this was not visible in the $16 \mathrm{~Hz}$ stimulation of Fig. $4.22 \mathrm{C}$. However, this indicates that after all adaptation does play a role in the processing of sounds delayed by the $S p n b^{q v-3 J / q v-3 J}$ mutation.

Similar to the spike rate reduction shown in panel $\mathrm{A}$ and the failure rate at $16 \mathrm{~Hz}$ in Fig. $4.22 \mathrm{D}$, the failure rate was higher in a range of sound intensities for mutant ICNs (see Fig. 4.23 D).

Furthermore, the vector strength is unaffected by changing the sound intensity as it is expected at $16 \mathrm{~Hz}$ modulation frequency (see Fig. 4.23 E), although the calculation of vector strength in general gets more imprecise the lower the sound level and therefore the less spikes are elicited.

With increasing modulation depth, an increase in spike rate can be observed in the PSTHs in Fig. 4.24 A, which is more pronounced in the wildtype ICNs that in those of Spnb ${ }^{q v-3 J / q v-3 J}$ mice. Also, a decrease in onset rate can be seen even for low modulation depth, while the steady-state rate looks similar.

The quantification of the absolute spike rates shows a relatively stable response up to $50 \%$ modulation depth and an increase in spike rate at 75-100\% modulation depth. The spike rate is significantly higher for ICNs from wildtype mice at modulation depths between $50-100$ \% (see Fig. 4.24 B). Decay ratio shown in Fig. 4.24 C is more comparable 

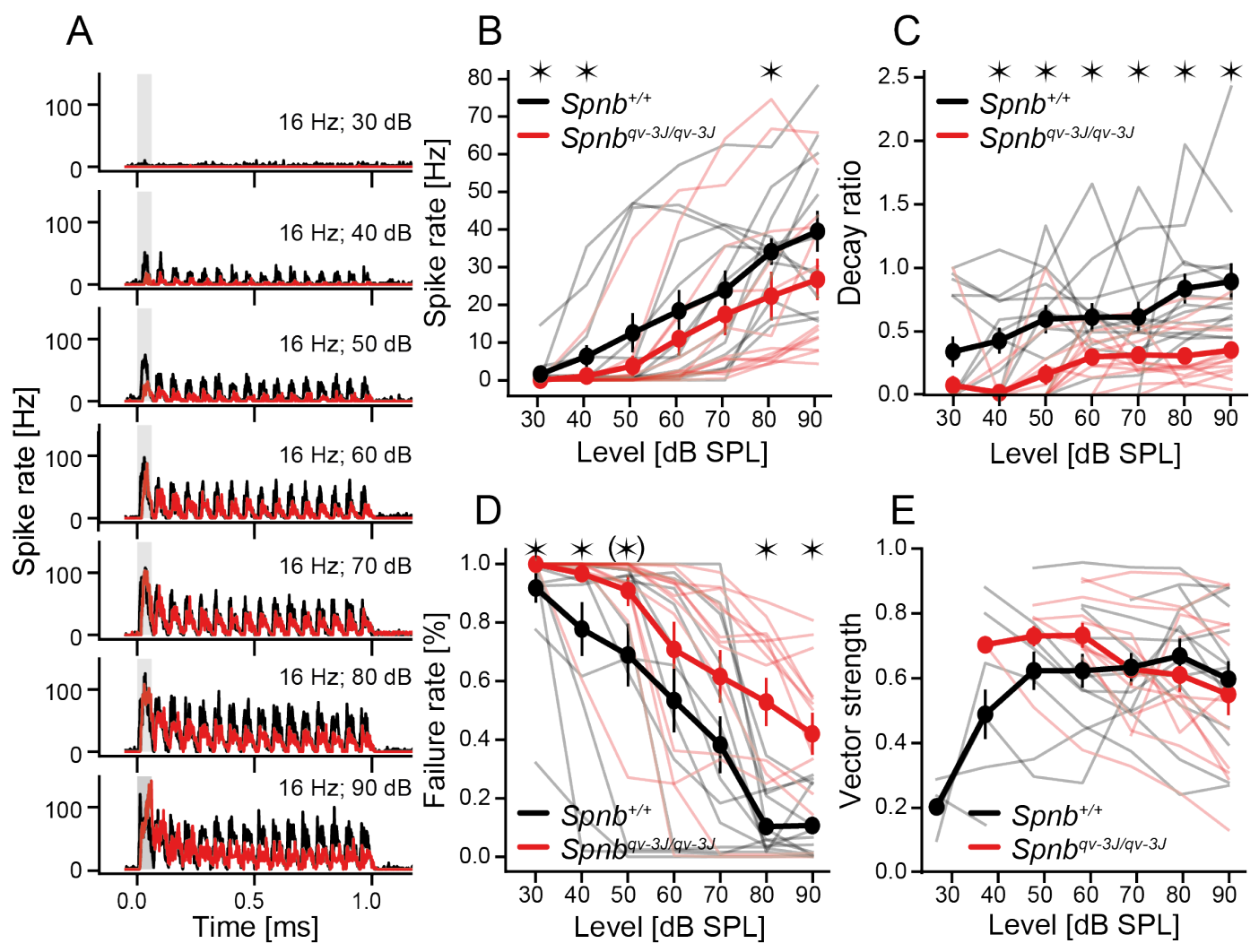

Figure 4.23.: Responses to amplitude modulated tones with varying sound intensities show a defect in spike rate of ICNs from $S p n b^{q v-3 J / q v-3 J}$ mice, depending on the sound intensity.

(A) Averaged PSTHs for each tested sound intensity at $16 \mathrm{~Hz}$ modulation frequency from 30$90 \mathrm{~dB}$. A reduction in spike rate can be seen for ICNs of $S p n b^{q v-3 J / q v-3 J}$ mice $(\mathrm{n}=13)$ compared to wildtype littermates $(\mathrm{n}=13)$. Duration of one cycle is marked in grey. (B) Rate modulation transfer function, which plots the averaged spike rate throughout the whole $1 \mathrm{~s}$ stimulation against the modulation frequency. Both genotypes increase their average spike rate with increasing sound level. The mutant ICNs seem to have a slightly lower spike rate and a significant difference was calculated at 30, 40 and $80 \mathrm{~dB}$. (C) The decay ratio, expressing the adaptation over time during amplitude modulated tones, is increased at all sound levels from 40-90 dB. (D) The failure rate correlates with the sound intensity as it is dropping linearly with rising sound levels and was higher in a range of sound intensities for mutant ICNs. (E) The synchronization modulation transfer function does not reveal any significant differences between ICNs from $S p n b^{q v-3 J / q v-3 J}$ mice compared to wildtype littermates as expected at $16 \mathrm{~Hz}$.

Circles in (B-D) represent mean \pm SEM. Asterisk represent significant differences, while asterisk in brackets represent no significance, but a difference which is still highly probable $(\mathrm{p}<0.08)$.

The full statistical analysis is shown in appendix B.

to the $16 \mathrm{~Hz}$ stimulation from Fig. $4.22 \mathrm{C}$, where it might be slightly reduced although none of the differences is significant.

The failure rate is mirroring the reduction is spike rate, by showing a significant increase at modulation depths between 50-100 \% (see Fig. 4.24 D). 


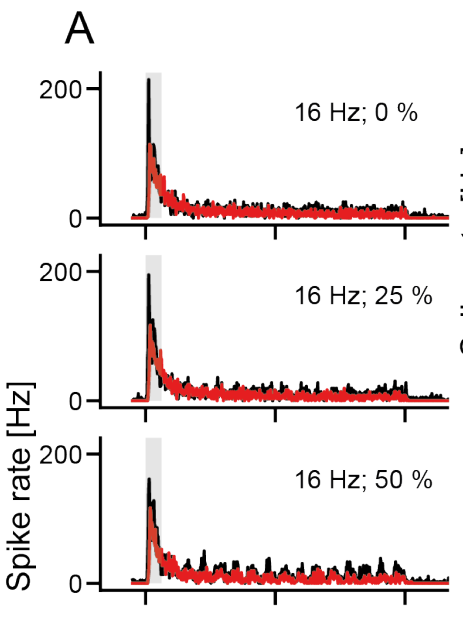

$B$

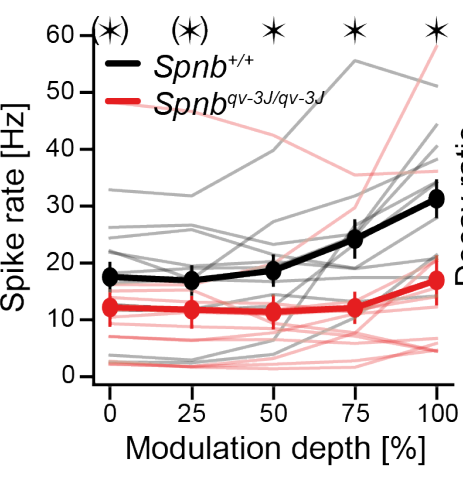

D
C

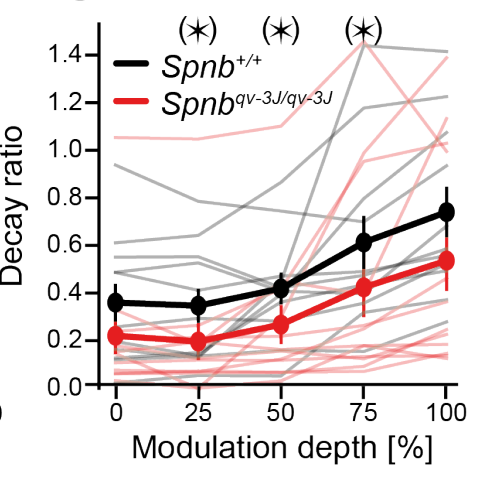

$\mathrm{E}$
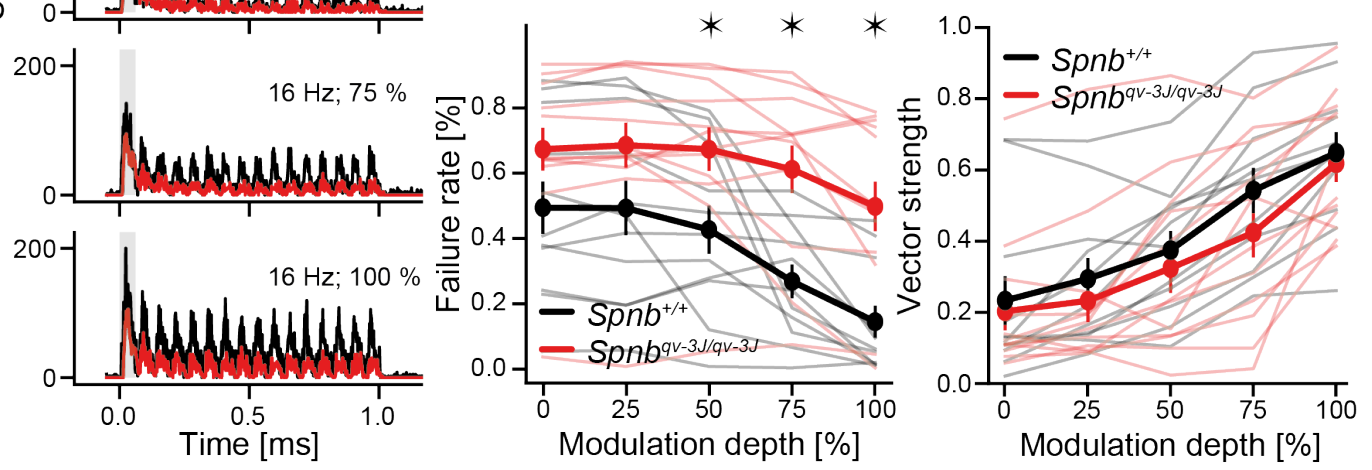

Figure 4.24.: Varying modulation depth in amplitude modulated tones seem to have an effect on spike rate and timing.

(A) Averaged PSTHs for each tested modulation depth at $16 \mathrm{~Hz}$ modulation frequency at dBOpt. For stronger modulation depths the spike rate of ICNs from Spnb ${ }^{q v-3 J / q v-3 J}$ mice $(\mathrm{n}=12)$ is weaker than that of wildtype littermates $(\mathrm{n}=11)$. Duration of one cycle is marked in grey. (B) Rate modulation transfer function, which plots the averaged spike rate throughout the whole $1 \mathrm{~s}$ stimulation against the modulation frequency. Spike rate is relatively stable from 0-50\% modulation depth and increases at 75-100\%. For mutant ICNs this increase is much shallower and spike rates are generally smaller than for wildtype ICNs. (C) The decay ratio, expressing the adaptation over time during amplitude modulated tone shows that the adaptation is generally stronger at low modulation depths and ICNs of Spnb ${ }^{q v-3 J / q v-3 J}$ mice seem to have an even more increased adaptation, although no difference is significant. (D) Failure rate plotted against modulation frequency. The failure rate is dropping at modulation depths from $50-100 \%$ for wildtype ICNs, consistent with the reduced spike rate. The failure rate of ICNs from Spnb ${ }^{q v-3 J / q v-3 J}$ mice is higher and gets only mildly reduced with higher modulation depths. (E) Synchronization modulation transfer function, which shows the vector strength as a measure of phase-locking, plotted against modulation frequency is strongly dependent on modulation depths, but similar for ICNs of Spnb ${ }^{q v-3 J / q v-3 J}$ mice compared with wildtype littermates, as expected at $16 \mathrm{~Hz}$.

Circles in (B-D) represent mean \pm SEM. Asterisk represent significant differences, while asterisk in brackets represent no significance, but a difference which is still highly probable $(\mathrm{p}<0.08)$. The full statistical analysis is shown in appendix B. 
Taking a closer look at the vector strength shows a good correlation between the modulation depth and the phase-locking, but no differences between ICNs from Spnb ${ }^{q v-3 J / q v-3 J}$ mice compared to wildtype littermates, as would be expected at $16 \mathrm{~Hz}$ modulation frequency (see Fig. 4.24 E).

In summary, the $S p n b^{q v-3 J / q v-3 J}$ mutation causes a time shift in response to sAM tones, which results in reduces spike rates and higher failure rates for low modulation frequencies and a reduced ability to phase-lock to the highest modulation frequencies possible for ICNs. Furthermore, spike rates show a strong correlation with sound intensity and modulation depth. Vector strength is comparable between wildtype and mutant ICNs at $16 \mathrm{~Hz}$ modulation frequency, but spike rates are reduced, especially at higher modulation depths. 



\section{Discussion}

We were interested in the functions of the ICC as a central nucleus of the auditory midbrain and investigated the central sound encoding in mouse models of auditory synaptopathy and neuropathy. With this we wanted to find out how sounds are perceived and how this perception might be altered in the investigated mouse models. For this reason, we used in vivo single unit recording from the ICC of mutant mice and compared them to wildtype littermates and previous data from in vivo single unit recordings from the AN. We wanted to find out more about the mechanisms of diseases associated with specific mutations to better understand the clinical picture of patients carrying these mutations. This might help diagnosing and treating different forms of auditory neuropathies. Furthermore we were interested in how the ICC processes different sound features when the input signals are slightly altered.

\subsection{Part I: Methodological considerations}

To get insights into auditory perception, we wanted to investigate the higher centers of the auditory pathway. Before the signals reach the ICC, a lot of auditory processing already took place. Still the diversity of responses and the influence of anesthesia is less than in the auditory cortex to be able to get meaningful results.

Four our studies on sound encoding in the ICC we used in vivo single unit recordings. We were taking great care on the separation between single units and multi unit activity to access the unique response patterns of singe units, undisturbed by integration of several neurons. Using single unit recordings we wanted to decrease the bias of neurons with high spike rates or short latencies, which might dominate multi unit responses. Although for this approach the number or recorded units is lower than for recording of multi unit activity, which might lead to a sampling bias, we can analyze neurons much more precisely. Single unit recordings have a much higher sensitivity to find neurons that are specialized in a given task and we selected as much as possible units that specialized to the features we were looking at. Still, we were not able to hit the specific specializations of every single unit as we for instance did not test ITD or ILD selectivity. In the goal of understanding general sound perception by recording single units, we miss the few fibers that bypass the ICC and we were not recording from inhibitory neurons.

We were classifying the recorded units into groups with similar responses and averaged them to get an overview about general functions of ICNs. Thereby we wanted to counteract the diversity of responses of ICNs. The single specializations in structure, physiology and function of an ICN could not be distinguished. It was not possible to tell from 
which units we recorded, where they get inputs from or which auditory task they are processing. Still, this convergence of inputs from many sources onto the ICNs is the reason why we could learn a lot about sound perception, but also makes interpretation of results difficult. In the downstream nuclei of the auditory pathway (AVCN, SOC or LL) a dedicated structure and function is required to perform a specialized task. The task specificity can no longer be separated due to the massive convergence of auditory signals in the ICC, which are compressed onto a single tonotopic organization. However, we were analyzing groups of neurons with similar responses to the same sounds to get insights into the coding of different sound features in the ICC.

For the analysis of most sound features, averaging the response of similar fibers was sufficiently meaningful. Single units from the auditory midbrain possess so-called stimulus specific adaptation (Ayala and Malmierca 2013). This term describes the decrease in spiking in ICNs to a repetitive sound, that is restored when a different sound is played. The IC is said to be the first stage in the auditory pathway where neurons have stimulus specific adaptation (Duque et al. 2018). It is likely that this phenomenon arises from cortical inhibitory circuits (Anderson and Malmierca 2012). A randomization of the stimuli, which we used for all our protocols, depresses stimulus-specific adaptation. However, for pure tone stimulation to create PSTHs this was not possible, and we had to exclude some neurons, which changed their spiking over time.

To improve the quality of our results, we were also considering, that different anesthetics used in in vivo experiments have different effects of the activity in the midbrain (Sloan 1998). Recording from awake animals would exclude the effects of anesthesia on brain activity, but it would also mean a huge strain for the animals, which we wanted to avoid. ABR recordings are less sensitive for different anesthetics (Smith and Mills 1989), so we could use a combination of ketamine and xylazine. However, it has been shown that ketamine, as an NMDA receptor antagonist, depresses activity in neurons more than other anesthetics. Therefore, for our ICC recordings we used an isoflurane inhalation narcosis. This narcosis can be adjusted very precisely as it wears of within minutes when the vaporizer is switched off. Hereby we were able to use the lowest isoflurane dose possible to keep the animal in a sleeping state, without depressing the neuronal activity more than necessary. In contrast to recordings from awake animals it has been shown, that ICNs might be able to phase-lock to higher modulation frequencies in awake animals than in anesthetized ones (Langner et al. 2002). By our approach we tried to minimize these effects but cannot rule out that differences between wildtype and mutant mice regarding phase-locking were concealed by the anesthesia.

Auditory research has been conducted on many different species and all sorts of mammals and different mouse lines. In general, genetically modified mouse models are a useful tool to study hereditary disease of humans. Significant similarities between the mouse and human inner ear structures have been demonstrated, making the mouse an appropriate animal model (Pennacchio 2003; Moser et al. 2013). They can be generated comparably easy and fast and enable us to investigate defects in auditory coding for specific mutations. Invasive electrophysiological recording techniques as well as morphological studies and in vitro examinations can be used on mice, which we could not use on human patients. Many common features in auditory coding between mammals have been 
discovered, although few species specificities exist (Fay 1994). For instance, in our experiments, the tone duration in a "long-pass" way was preferably coded by "sustained" units in $\mathrm{C} 57 \mathrm{Bl} / 6$ mice $\left(S p n b^{q v-3 J / q v-3 J}\right)$ than in CBA mice (Otof $\left.{ }^{I 515 T / I 515 T}\right)$. Furthermore, we need to consider that mice have a much higher frequency hearing range than humans, relying less on phase-locking and ITDs. Nevertheless, phase-locking to the envelope of sounds is well possible in both mice and humans. To exclude strain specific peculiarities, we always compared our mutant mice to wildtype littermates. Therefore, we could focus on the effects of the different mutations on auditory coding. Only for the Otof Pga/Pga mice no wildtype littermates were available, as they were bread homozygously.

\subsection{Part II: Sound encoding in the ICC of mutant mice}

\subsubsection{Frequency coding}

The auditory system is well structured, following a tonotopical organization throughout all parts up to the auditory cortex, starting at the basilar membrane where high frequencies are located at the base of the cochlea and low frequencies are located at the apex. This tonotopical organization is also present in the ICC where low frequencies are located dorsolaterally and high frequencies ventromedially (Merzenich and Reid 1974).

We investigated the influence of the mutations Otof ${ }^{I 515 T / I 515 T}$ and Spnb ${ }^{\text {qv-3J/qv-3J leading }}$ to auditory synaptopathy and neuropathy, respectively on the tonotopical organization in the ICC. Therefore we analyzed the distribution of CFs to get an impression of the tonotopy in the ICC. In previous studies on sound encoding in SGNs of these mice it has been shown that both Otof ${ }^{I 515 T / I 515 T}$ and Spnb ${ }^{q v-3 J / q v-3 J}$ mice had comparable CF distributions in the SNGs between wildtype and mutant mice (Strenzke et al. 2016; Yamanbaeva 2017). The spectrin protein is only present in neurons, while otoferlin is localized in the IHC ribbon synapse. Therefore, no effects on cochlear organization or on the frequency-place code of the basilar membrane would have been expected. In the ICC, tonotopic organization was intact as well and distributions of CFs were comparable in all wildtype and mutant mouse lines.

Furthermore, we classified frequency response of each ICN according to Ramachandran et al. 1999 into "V-shaped", "I-shaped" and "O-shaped" units. All classes of FRAs were present in equal numbers and no differences between genotypes in Otof ${ }^{I 515 T / I 515 T}$ and $S p n b^{q v-3 J / q v-3 J}$ mouse lines were found. An alternative classification was proposed by Egorova et al. 2001. Hereby ICNs were classified into four categories. Class I neurons have similar FRAs as SGNs, with asymmetric tuning curves, steep high-frequency slopes and shallow low-frequency slopes. These would correspond to "V-shaped" units. Class II neurons, which are dominated by inhibitions, show symmetrical inhibitory areas with steep slopes on both sides and would correspond to both "I-shaped" and "O-shaped" neurons. Class III neurons have weak inhibition and symmetric and shallow tuning curve slopes and would also correspond to "V-shaped" neurons. Class IV neurons are composed of neurons with a complex tuning curve, which might have multiple CFs. Units of Class IV were occasionally encountered in this thesis, but were classified as "other" 
units. All the reported different FRA shapes were observed in this study, although class I and III units could not be clearly distinguished and were both classified as "V-shaped" units.

In contrast, in Otof ${ }^{P g a / P g a}$ mice the classification of FRAs was not possible and the frequency-coding in the ICC seems to be impaired. We could not investigate distributions of CFs, but FRAs tended to be broader and thresholds were strongly increased. In otoferlin knock-out mice, which are similar to Otof ${ }^{P g a / P g a}$ mice, it has been shown that cochlear amplification, measured by recording distortion products of otoacoustic emissions (DPOAEs) is degraded with age, which could be a cause for the elevated thresholds (Stalmann 2015). However, this degradation did not occur before 24 weeks of age. DPOAEs of Otof ${ }^{P g a / P g a}$ mice were not recorded at older ages, but it is unlikely that major impairments in cochlear amplification were present at the age of 7 weeks, when we recorded single units from the ICC. We suggest that the broadened FRAs of Otof ${ }^{P g a / P g a}$ mice result from a compensation to the reduced vesicle exocytosis. The reduced input into the ICC could lead to more input fibers from a broader frequency range being recruited to a single ICN as a compensatory mechanism in increasing central gain.

We conclude that mutations which do not cause cochlear malformation or change neuronal connections do not impair frequency coding in the cochlea or the AN. If too less input reaches the ICC it could react with increasing the number of inputs to a single ICN (Otof $\left.{ }^{P g a / P g a}\right)$. For mutations with considerable residual spiking, which affect spike rates (Otof ${ }^{I 515 T / I 515 T}$ ) or spike timing (Spnb $\left.{ }^{q v-3 J / q v-3 J}\right)$ the place-code for tonotopy is not influenced.

\subsubsection{Spontaneous rate and intensity coding}

In the cochlea, apart from the tonotopic organization due to the properties of the basilar membrane, further structural organization is present with SGNs having different properties including a specific spontaneous rate, dynamic range as well as spike rate and latency. Low-spontaneous rate fibers with high thresholds contact the IHCs preferentially at the modiolar side, while high-spontaneous rate fibers with low thresholds contact IHCs at the pillar side (Liberman 1982b; Merchan-Perez and Liberman 1996).

A similar structure in spontaneous rate distribution or threshold distribution is not known from the ICC. The spontaneous rate of ICNs is generally very low with many neurons having no spontaneous rate at all (Ehret and Moffat 1985; Ehret and Merzenich 1988b; Wagner 1994) even in awake animals (Ryan and Miller 1978). Still we were interested how the intensity information, which in SGNs is dependent on the spontaneous rate is affected in the ICC of our mouse models.

Spontaneous rates were normal in SGNs of Otof ${ }^{1515 T / I 515 T}$ mice and reduced in SGNs of Spnb $b^{q v-3 J / q v-3 J}$ mice. Interestingly, for both mutations the spontaneous rate was similar in ICNs as in SGNs. This means spontaneous rate in ICNs of Otof $1515 T / I 515 T$ mice was normal while in ICNs of $S p n b^{q v-3 J / q v-3 J}$ mice it was reduced. In $S p n b^{q v-3 J / q v-3 J}$ mice the spontaneous exocytosis of synaptic vesicles should be completely normal. However 
the $S p n b^{q v-3 J / q v-3 J}$ mutation disturbs sodium channel clustering in the AIS, which might cause fewer action potentials being elicited upon a single spontaneous release event (Kole et al. 2008). In the ICNs it is likely that the spontaneous rate is also dependent on inputs. One possible factor might be that the reduced number of spontaneous action potentials in the SGNs of $S p n b^{q v-3 J / q v-3 J}$ mice is transmitted to the ICC and therefore causes a reduced spontaneous rate in ICNs.

In contrast to SGNs where rate-level functions follow a monotonic sigmoid function, ratelevel functions of single units in the ICC can have monotonic, non-monotonic or complex shapes. In cats the percentage of single units in the IC responding in a monotonic way to pure tones was below $10 \%$ (Ehret and Merzenich 1988a; Aitkin 1991) and approximately $35 \%$ in mice (Ehret and Moffat 1985). In our study this percentage was even higher with approximately $60 \%$ monotonic neurons and $40 \%$ non-monotonic units. Furthermore, it has been shown that rate level functions in response to tones can be different than those in response to noise, where the fraction of monotonic units was higher in cats (Ehret and Merzenich 1988a; Aitkin 1991). However, we only used pure tone and sAM tone stimulation.

The actual discharge rate seems to play less a role for intensity-coding in the ICC than in the AN as already proposed by Ehret and Merzenich 1988a. This hypothesis is supported by our findings. The Otof ${ }^{1515 T / I 515 T}$ mice had shallower rate-level functions in the SGNs with a normal dynamic range. In rate-level functions of the ICNs, this did not affect mean or maximal spike rates. Instead, the Otof ${ }^{I 515 T / I 515 T}$ mutation lead to a reduction in dynamic range for both monotonic and non-monotonic fibers. This smaller dynamic range despite of normal spike rates might be a consequence of a compensatory increase in gain. In $S p n b^{q v-3 J / q v-3 J}$ mice, delay of spiking did not have any effect on rate-level functions in ICNs.

However, we did not take into account that FSL coding, which was impaired in ICNs of Spnb ${ }^{q v-3 J / q v-3 J}$ mice, also contributes to intensity coding. Generally, with higher sound intensities the FSL gets shorter and the "primary-like" PSTH gets more defined in SGNs (Klug et al. 2000). Still, in observations of PSTHs from ICNs at different sound intensities, FSL seems to play less of a role than in SGNs.

\subsubsection{Duration tuning}

Recent studies suggested the presence of neurons in the ICC coding specifically tones with a certain tone length. In a study on sound encoding in the ICC, $55 \%$ of ICNs in mice had long- short- or band-pass filter characteristics (Brand et al. 2000).

In this thesis we were interested in how mouse models for auditory neuropathies affect tone duration coding in the ICC. We were especially interested in the responses of Otof I515T/I515T mice to sound duration. As these mice have an impaired adaptation to continuous sounds in SGNs we expected them to have an impaired coding of longer tones compared to shorter ones. 
However, in our study we only found long-pass or unfiltered units, but neither short-pass nor band-pass units, not even in wildtype mice. The only distinction we could make was into "onset" units that spiked at the onset of tones, independent on the tone length and into "sustained" units that spiked throughout the whole tone for any tone length.

In Otof ${ }^{I 515 T / I 515 T}$ mice with a CBA background we could not observe any correlation between the filtering of units and their response pattern. However, in Spnb ${ }^{q v-3 J / q v-3 J}$ mice with a $\mathrm{C} 57 \mathrm{Bl} / 6$ background, long-pass units of both mutant and wildtype mice seemed to respond preferentially with a "sustained" response.

In ICNs of Otof ${ }^{I 515 T / I 515 T}$ mice we could not observe any difficulties in coding tone duration. However, in ICNs from $S p n b^{q v-3 J / q v-3 J}$ mice we had a higher minimal tone length needed to elicit a response than in $S p n b^{+/+}$mice. This seemed to be caused by a difference in the fraction of "sustained" neurons, as the "onset" units nearly all had minimal tone length of $8 \mathrm{~ms}$, the shortest measured tones. Although the difference of minimal tone length needed to elicit a response in the fraction of "sustained" units is not significant, a trend towards longer tones can be observed. This reduced ability to respond to short tones might be explained by the ICNs of $S p n b^{q v-3 J / q v-3 J}$ mice needing longer integration times due to the delay of spiking and reduced spike rates, which might function as a compensatory mechanism.

\subsubsection{Spike rates and spike timing}

We investigated the effects of the different mutations on the spiking over time, visualized by PSTH shapes, including spike rates and spike timing in the ICC. In the AN and AVCN, comparisons of PSTH shapes from in vivo electrophysiological recordings are very useful. Recorded units from SGNs can be distinguished from different neuron types in the AVCN by their response pattern (Bourk 1976; Rhode et al. 1983). SGN responses to pure tones have a "primary-like"-shaped PSTH with a sharp peak at the onset of the tone and a reduction of spike rate to a steady-state rate during stimulation. This "primary-like" PSTH mimics the release of neurotransmitters from the ribbon synapse (Taberner and Liberman 2005). The sharp onset peak resembles the release of the RRP, while the steady-state rate is evoked by the continuous replenishment and release of synaptic vesicles.

In contrast, PSTH shapes of ICNs have very diverse shapes without any known correlation between response pattern and neuron type. However, averaging all measured units from the ICC also results in a "primary-like" shaped PSTH. We were calculating and averaging peak and steady-state rates for all measured ICNs. Still, estimates of spike rates calculated by this approach must be taken with care, as these averages were very outlier sensitive.

Both Otof ${ }^{I 515 T / I 515 T}$ and Spnb ${ }^{q v-3 J / q v-3 J}$ mice had a reduction in steady-state rate in SGNs, but neither of them resulted in a reduction of spike rate of the sustained response in ICNs. However, peak rate was reduced in ICNs of both Otof I515T/I515T and $S p n b^{q v-3 J / q v-3 J}$ mice, while peak rates in SGNs were reduced in $S p n b^{q v-3 J / q v-3 J}$ mice and showed a trend towards a reduced peak rate in Otof $f^{I 515 T / I 515 T}$ mice. Our study suggests 
that the onset components and the sustained components of ICN responses code for different auditory features and do not arise directly from the peak and steady-state rates of the SGNs. It has already been suggested that it is generally necessary to separate the analysis of onset and late response component in the IC (Heil 1998). Onset responses likely rely mainly on coincidence detection while steady-state rates are dependent on the integration time of a neuron. A compensatory increase in central gain in the mutant mice might lead to a longer integration window to compensate spike rates but leading to more jittered onset responses. Further indications for this conclusion provide the AVCN neuron recording from $S p n b^{q v-3 J / q v-3 J}$ mice. Already at the second stage of the auditory pathway the steady-state response does not differ between wildtype and mutant mice anymore in GBCs and stellate cells.

In addition to that, we investigated the effect of the two mutations Otof $f^{I 515 T / I 515 T}$ and $S p n b^{q v-3 J / q v-3 J}$ on spike timing of the FSL, as hearing crucially depends on short first spike latencies and low jitter (Kopp-Scheinpflug and Tempel 2015). In Otof ${ }^{I 515 T / I 515 T}$ mice the reduction in peak rate of ICNs correlates with an increased variance of FSL. In ICNs of $S p n b^{q v-3 J / q v-3 J}$ mice the FSL is delayed, but not more jittered in the slow stimulation condition. However, variance of FSL was generally higher in ICNs of $S p n b^{q v-3 J / q v-3 J}$ mice than in those from Otof ${ }^{I 515 T / I 515 T}$ mice. For quiverer-4J mice it has been reported, that the "quivering" mutation leads to an increased FSL and greater jitter in AVCN neurons (Kopp-Scheinpflug and Tempel 2015). However, the latency difference in AVCN neurons of our quiverer-3J mice was to subtle to reach significance (Yamanbaeva 2017). However, in our recordings from ICNs the delay of spiking is obvious.

A major impairment in the sound encoding in the ICC of $S p n b^{q v-3 J / q v-3 J}$ mice was revealed when stimulating with a higher repetition rate of $5 \mathrm{~Hz}$. This led to a dramatic increase in FSL, which was much longer compared to the $2 \mathrm{~Hz}$ stimulation conditions. Furthermore, at $5 \mathrm{~Hz}$ the variance of FSL was strongly more jittered. This reveals a major impairment in precision of spiking, which is strongly dependent on stimulus repetition rate.

\subsubsection{Compensatory mechanisms by increasing central gain}

There is extensive evidence that the IC is able to increase central gain as a compensatory mechanism for reduced afferent input. For instance, a deletion of $95 \%$ of all SNGs by ouabain treatment leads to considerable and relatively normally tuned responses in the ICNs (Chambers et al. 2016). Similar things could be observed in chinchillas treated with carboplatin, which selectively destroys IHCs, although here the increase in central gain was mainly in the auditory cortex and only partially in the IC (Salvi et al. 1999). In this model it has been shown that neural synchrony in the AN and IC are intact despite significant reductions in neural input (Cowper-Smith et al. 2010) and ICN thresholds were generally considerably lower than the strongly elevated ABR thresholds (Harrison 1998). Based on these findings, we wanted to know to what extent a compensation in the ICC to a defect as intense as the Otof ${ }^{P g a / P g a}$ mutation is possible. 
The recordings from ICNs of Otof ${ }^{P g a / P g a}$ mice revealed broadened FRAs, strongly increased pure tone thresholds and drastically reduced spike rates. The increased thresholds made it impossible to tell by the rate-level function if a unit would be monotonic or non-monotonic. Additionally, the spike rates were severely depressed and long recovery times were needed to elicit spiking at all. This phenotype in the ICNs is similar to the recordings from the SGN, suggesting that the Otof ${ }^{P g a / P g a}$ mutation could not even be partially compensated by increase in central gain.

This compensatory mechanism described before seems to be only possible under certain conditions. In the Chambers et al. 2016 study most SGNs were lost, while in Otof Pga/Pga mice SGNs are intact but transmit only few action potentials. We hypothesize that compensation in the IC is easier for missing input than for reduced input. Fewer, but normal fibers can be compensated by increase in central gain but the reduced responses of many fibers with impaired input cannot be improved by processing in the brainstem.

\subsubsection{Forward masking}

Forward masking is an interesting stimulus paradigm, which - in SGNs - analyzes the replenishment of synaptic vesicles, which takes place during the silent gap (Harris and Dallos 1979; Meddis and O'Mard 2005). Hereby, firstly a masker tone is played to deplete the pool of readily-releasable vesicles, followed by a silent interval of variable length and a probe tone to see how many vesicles could be reformed during the silent interval. In contrast to a pure gap detection task, no continuous background noise is present. Here, differences in the coding of silent gaps in noise have been reported between excitatory and inhibitory units in ICNs (Wilson and Walton 2002). While excitatory units detect gaps in white-noise best in quiet, inhibitory units profit from additional continuous background noise for their ability to detect gaps in the foreground noise burst stimuli. This indicates that there might be further contributions in gap detection like the influence of inhibitory neurons. Although forward masking can provide useful insights into gap detection as well, its main purpose is in analyzing replenishment of synaptic vesicles.

For Otof I515T/I515T SGNs forward masking revealed a clear deficit in the IHC ribbon synapse with reduced otoferlin levels, where depression of spiking was increased and recovery from forward masking was delayed. In contrast, the delay of spiking caused by the Spnb ${ }^{q v-3 J / q v-3 J}$ mutation did not lead to any differences in forward masking in SNGs, which is no surprise, as the mutation does not affect the vesicle replenishment in the IHC ribbon synapse but can first be found in the SGNs.

In the ICC, forward masking is similarly impaired in Otof ${ }^{I 515 T / I 515 T}$ mice as in SGNs, with an increased depression of spiking and an elevated minimal silent interval needed to elicit a probe response. This leads to the conclusion, that forward masking is only barely processed within the brainstem, as it is similar between SGNs and ICNs.

In line with this, delay of spiking did not influence forward masking, as wildtype and mutant mice in the $S p n b^{q v-3 J / q v-3 J}$ strain showed comparable results. The delay of spiking caused by the $S p n b^{q v-3 J / q v-3 J}$ mutation only affects spike timing in the forward masking task, but as we designed the forward masking analysis in a way that spikes are detected 
independent of the delay, this causes no differences in depression of spiking or minimal silent interval. Depression of spiking in a forward masking task seems to be unaffected by threshold elevations and spike delays. It has been shown that gap detection is generally not impaired by moderate high frequency sensorineural hearing loss (Walton et al. 2008). A defect in forward masking seems to be specific for impaired vesicle replenishment.

In the Otof ${ }^{I 515 T / I 515 T}$ mice, this replenishment phenotype lead to a deficit in gap detection in behaving animals. Thereby, mice were trained to drink water when continuous noise was present and to avoid drinking water when the noise was interrupted by gaps (Strenzke et al. 2016). We do not expect Spnb ${ }^{q v-3 J / q v-3 J}$ mice to have a behavioral deficit in gap detection as forward masking was normal.

\subsubsection{Precision of phase-locking in response to sAM tones}

Phase-locking, the ability of neurons to fire action potentials synchronized to the phase of a pure tone stimulus or to the envelope of a sAM tone, is a very interesting phenomenon in auditory coding and an important property needed for speech perception (Moore 2008). Therefore, we were interested how a delay of spiking caused by the $S p n b^{q v-3 J / q v-3 J}$ mutation would affect phase-locking in the ICC. Furthermore, in regard of the poor speech perception of patients with an $O T O F^{1515 T / I 515 T}$ mutation, we were very much interested how the ICNs of Otof ${ }^{I 515 T / I 515 T}$ mice would process amplitude modulated sounds.

Generally, phase-locking in the ICC of mice is only possible to low frequencies. Most studies using mice as model organisms used AM tones in this regard. Similarly, we were stimulating with sAM tones with a carrier frequency at $\mathrm{CF}$ and a modulation frequency between 2 and $512 \mathrm{~Hz}$. In our hands phase-locking in ICNs to the envelope of these tones was possible up to $64-128 \mathrm{~Hz}$ modulation frequency. Additionally, we could show that phase-locking is strongly dependent on modulation depth, but almost not dependent on sound intensity.

In Otof ${ }^{I 515 T / I 515 T}$ SGNs, phase-locking to transposed tones, presented with a modulation frequency of $500 \mathrm{~Hz}$, showed a trend towards smaller vector strength, although a significant difference could not be shown by statistical testing due to too small recorded units. Surprisingly, in ICNs the Otof ${ }^{I 515 T / I 515 T}$ mutation did not affect precision of spiking, as the vector strength, a measuring value for temporal precision of phase-locking, was not different from Otof ${ }^{I 515 T / I 515 T}$ ICNs compared to Otof ${ }^{+/+}$littermates. However, vector strength only measures how well the spikes are synchronized to the phase but not how reliable they are elicited.

In ICNs of $S p n b^{q v-3 J / q v-3 J}$ mice, which had an increased FSL and jitter, phase-locking was impaired. More precisely, phase-locking to low modulation frequencies was normal, but the maximal modulation frequency where phase-locking was still possible was reduced. This difference between vector strength of wildtype and mutant ICNs is most prominent between $64-128 \mathrm{~Hz}$. We think that the rate-dependency of spiking, which gets worse with faster stimulation, is affecting phase-locking in this range. Possibly, the jitter of spiking gets too high to be able to phase-lock to the envelope of sAM tones any more. 


\subsubsection{Spike rates in response to $\mathrm{sAM}$ tones}

Synchronizing the response to the envelope of sAM tones (phase-locking) is not the only way to encode sAM tones. A transition into a rate-based or a space-based envelope coding is likely happening in the brainstem (Joris et al. 2004). Therefore, we did not only investigate the synchronous firing of ICNs in response to sAM tones, but also differences in spike rates.

In response to $50 \mathrm{~ms}$ pure tones presented at 2, 5 and $10 \mathrm{~Hz}$, SGNs of Otof ${ }^{I 515 T / I 515 T}$ mice had a use dependent reduction of spike rate with an increased adaptation to continuous sounds and a reduced steady-state rate, dependent on the stimulus frequency. As mentioned before, in ICNs this was not the case. An impaired adaptation to pure tones could not be shown and steady-state rate was not different between wildtype and mutant ICNs. However, in response to sAM tones we could show a strongly impaired adaptation between $2-64 \mathrm{~Hz}$ modulation frequency. This is the same range of frequencies where phase-locking was possible to the envelope of sAM tones.

This is an interesting phenomenon, where we could observe at high modulation frequencies of $>512 \mathrm{~Hz}$ that the PSTH looks similar to the one generated with pure tone stimulation. We seem to have a decreased onset response to the whole stimulus, but we do not have an increased adaptation or a reduced steady-state rate. The duration of the stimulus does not affect the PSTH shape as well. Stimulation with a modulation depth of $0 \%$, which is literally a $1 \mathrm{~s}$ long pure tone results in a comparable PSTH as the $200 \mathrm{~ms}$ pure tone or the $512 \mathrm{~Hz}$ sAM tone with $100 \%$ modulation depth.

As we showed before, only onset rate is reduced in pure tone stimulation of SGNs in Otof ${ }^{I 515 T / I 515 T}$ mice. We therefore hypothesize that sAM tones with low modulation frequencies are encoded in the ICC as a series of rapidly repeating pure tones. The higher the repetition rate of the stimulation, or in this case the higher the modulation frequency, the stronger is the adaptation. This is in line with the previously reported use dependent reduction of spike rates, which could be shown in single unit recordings from SGNs when stimulating at higher repetition rates.

In summary, sAM tones that exceed the phase-locking capabilities of the ICNs seem to be perceived by them as pure tones, while sAM tones with lower modulation frequencies elicit an onset response in each phase. The Otof ${ }^{I 515 T / I 515 T}$ mutation does not affect the adaptation of pure tones or high frequency sAM tones, but adaptation in low frequency sAM tones is strongly impaired.

Additionally to the adaptation, we had a look on absolute spike rates and failure rates. In ICNs from Otof ${ }^{I 515 T / I 515 T}$ mice, the absolute spike rates were reduced compared to wildtype littermates at modulation frequencies up to $256 \mathrm{~Hz}$. The failure rate was increased accordingly. The failure rate hereby provides additional information on entrainment, a measure of the extent to which spikes are generated in each and every stimulus period (Rhode and Smith 1986; Joris et al. 1994).

Another interesting observation was made investigating the responses of ICNs of wildtype mice to sAM tones with different modulation depth. In the way the sAM tones 
are calibrated, an increase in modulation depth leads to a reduction in absolute sound intensities. Still, the ICN's spike rates are increasing with higher modulation depth. This might be explained by a synchronization of the synaptic transmission by synchronized vesicle release, which is caused by the sharpening of the single phases within one sAM tone.

\subsection{Part III: Human auditory synaptopathies and neuropathies}

Sensorineural hearing loss, the most common hearing impairment, is caused by disorders of the cochlea or the auditory nerve (Smith et al. 2005). Mostly, cochlear amplification is impaired due to an impaire electromotility of OHCs, which leads to impaired intensity sensitivity, but normal temporal processing (Patuzzi et al. 1989; Zeng et al. 2005). This can often be treated or at least partially compensated by external amplification of sounds with hearing aids.

Hearing impairments with normal sound amplification by OHCs, but abnormal neural sound encoding is known as auditory neuropathy or auditory synaptopathy when proteins of the IHC ribbon synapse are affected (Starr et al. 1996; Moser and Starr 2016). This can cause an impairment in timing related perception. Affected sound features can be pitch discrimination at low frequencies, detection of speech in noise or impaired sound localization. Clinically that results in impaired ABRs, but preserved OAEs and cochlear microphonics. Pure tone audiograms are often less affected than it would be expected by the speech perception deficit. Typically, patients with auditory neuropathy do not benefit from conventional hearing aids. As the cochlear amplification is intact, making sounds louder does not improve speech comprehension.

To be able to find therapies for these patients, the cellular mechanisms need to be understood and genetic causes need to be discovered. Congenital auditory neuropathies can be caused by numerous gene defects and have many different manifestations from mild hearing impairments to profound deafness. While the cause of the mutation is barely identifiable by hearing tests (audiogram, ABR and DPOAE), it plays a major role in predicting the therapeutic benefits.

These hearing disorders can be divided into non-syndromic auditory neuropathies, which specifically affect hearing function, and syndromic auditory neuropathies, where the hearing impairment is a symptom of a syndrome that affects not only hearing function.

Non-syndromic congenital auditory neuropathies are caused by protein defects of haircell specific proteins, of proteins specific for auditory neurons like SGNs or neurons from higher auditory nuclei. Prominent examples are mutation in the gene coding for the vesicular glutamate transporter-3 (VGLUT3), which causes DFNA25 and leads to profound deafness (Ruel et al. 2008). Another example is a mutation in the gene coding for pejvakin, which causes DFNB59, which is a mixed form with both auditory neuropathy 
and abnormal OAEs (Delmaghani et al. 2006, Collin et al. 2007). Furthermore, numerous studies have been reported on non-syndromic auditory neuropathies being caused by mutations in the OTOF gene (see below).

On the other hand, syndromic auditory neuropathies are often caused by mutations in proteins important for maintenance of neuronal function. As the hearing system requires constant and intensive action potential firing, small defects in neuronal function that are not life-threatening, for instance affecting metabolism, turnover or homeostasis of neuronal proteins, will likely affect hearing function. An interesting example is the CAPOS syndrome (cerebellar ataxia, areflexia, pes cavus, optic atrophy and sensorineural hearing impairment). These patients have a mutation in the neuron specific $\alpha$-subunit of the sodium-potassium-ATPase- $\alpha 3$. This results in present OAEs and cochlear microphonics but abnormal ABRs and poor speech perception, especially in noise (Tranebjærg etal. 2018). The result of this mutation might be an impaired propagation of electrical impulses along the SGNs. Another example are the mutations in the SPNB gene coding for $\beta \mathrm{IV}$-spectrin investigated in this study. Moreover, clinical features of auditory neuropathy can develop in neurological disease like Friedreichs ataxia (Rance et al. 2008), Guillain-Barré syndrome (Nelson et al. 1988) or multiple sclerosis (Schweitzer and Shepard 1989).

\subsubsection{Human patients with non-syndromic auditory synaptopathy DFNB9}

The autosomal recessive non-syndromic prelingual deafness DFNB9 is caused by mutations in the OTOF gene coding for otoferlin (Shearer and Smith 2008). It is characterized by normal OAEs and cochlear microphonics, at least in young patients, but absent or low amplitude ABRs and CAPs (Rodríguez-Ballesteros et al. 2003, Santarelli et al. 2009). Treatment for patients with DFNB9 can only be provided by cochlear implants, which can restore CAPs and speech perception to a certain extent (Santarelli et al. 2015). Although initially normal, OAEs in patients with DFNB9 often get degraded with age, which makes diagnosing this hearing impairment difficult (Shearer and Smith 2008). This degradation of OAEs with age could be also be reproduced in otoferlin knock-out mice (Stalmann 2015).

That DFNB9 is caused by OTOF mutations has been shown in screenings of auditory neuropathies in many different populations, especially prominent in the Spanish population (Rodríguez-Ballesteros et al. 2008), but was also found in Pakistan (Choi et al. 2009), Turkish (Tekin et al. 2005) or Chinese populations (Wang et al. 2010). Most patients with DFNB9 are clinically deaf. However, hearing function was not completely abolished as using power hearing aids in those patients could improve hearing function, although it was still below what is needed for speech perception (Santarelli et al. 2015). With cochlear implants, understanding speech was again possible for many patients.

Of the DFNB9 patients, different manifestations have been reported, ranging from profound deafness to moderate or mild sensorineural hearing loss (Zadro et al. 2010). More severely affected patients can have progressive hearing loss or auditory dys-synchrony 
(Yildirim-Baylan et al. 2014). Milder cases may have residual hearing with an impaired speech perception (Cheng et al. 2017). Several mutations associated with DFNB9 have a temperature-sensitive auditory neuropathy, where hearing function is lost upon elevation of the body temperature (Marlin et al. 2010; Zhang et al. 2016), one of which is the I515T mutation investigated in this study. The different mutations in the OTOF gene, which have been reported are distributed throughout the whole gene locus and all 6-7 C2 domains of otoferlin and may affect several of its functions (Pangršič et al. 2012). Still despite the different manifestations, the clinical phenotypes of patients with OTOF mutations are generally similar, and it has been suggested that the amount of residual otoferlin at the IHC ribbon synapse active zone might be a crucial factor for the severity of hearing impairment (Strenzke et al. 2016).

In this study we investigated a mouse line with an Ile515Thr substitution in the Otof gene. This mutation was discovered in human patients with temperature-sensitive auditory neuropathy (Mirghomizadeh et al. 2002; Varga et al. 2006). They had hearing impairments consisting of near normal hearing thresholds, impaired adaptation to continuous sounds, poor speech perception and auditory fatigue (Wynne et al. 2013). Although the temperature-sensitivity was only partially reproduced in mice, the mouse line is mirroring the hearing impairments and serves as a model for moderate DFNB9 (Strenzke et al. 2016).

By investigating central sound encoding in the Otof ${ }^{I 515 T / I 515 T}$ mouse line we wanted to find out why human patients have an impaired speech perception despite normal hearing thresholds and if it might be possible to improve hearing function in DFNB9 patients with residual hearing. Firstly, we needed to understand the mechanisms why speech perception, especially in noise is impaired.

Therefore, we were very much interested how the ICNs of Otof ${ }^{I 515 T / I 515 T}$ mice would process amplitude modulated sounds. These sounds might give us insights into envelope coding, which is a very important property to encode speech (Swaminathan and Heinz 2012). Our results indicate that the strong adaptation in Otof ${ }^{I 515 T / I 515 T}$ ICNs in response to sAM tones, but not in response to pure tones likely contributes a lot to the speech perception deficit. Furthermore, the impaired coding of silent gaps and depressed spiking of tones after gaps in the forward masking stimulus, which cannot be compensated in the ICC may be another major cause for impaired speech perception in patients with an $O T O F^{I 515 T / I 515 T}$ mutation.

\subsubsection{Human patients with syndromic auditory neuropathy}

Until recently, human patients with auditory neuropathy caused by mutations in the $S P N B$ gene have not been reported. Based on the characterization of "quiverer" mice, a phenotype of human patients would likely include motor, cognitive and sensory dysfunctions. With a disintegration of nodes of Ranvier, the quivering-3J phenotype is comparable to demyelinating neuropathy or axonal degeneration (Waxman 2006; Frohman et al. 2006). Therefore, due to the severe motor problems those patients would likely be diagnosed with hereditary motor and sensory neuropathy (Charcot-Marie-Tooth disease) 
where the exact genetic cause is usually not known. In case reports, Charcot-MarieTooth disease can lead to hearing impairments with normal sound detection, delayed ABR waveforms and impaired processing of auditory temporal cues and speech comprehension (Rance et al. 2012), a comparable phenotype to the expected Spnb ${ }^{q v-3 J / q v-3 J}$ hearing dysfunction in humans.

With increasing genetical testing of hereditary disease, the first pathogenic variants of SPNB in human patients were reported. One patient described by Knierim et al. 2017 had myopathies and auditory neuropathies similar to those seen in the more severe forms of quivering mice, including absent ABRs but normal OAEs. The second reported patient had a $S P N B$ mutation causing a severe neurological syndrome with congenital hypotonia, intellectual disability and motor axonal as well as auditory neuropathy (Wang et al. 2018). In this study they reported that the phenotype was similar to quiverer-4J mice and found out, that $\beta 1$-spectrin can compensate clustering sodium channels for $\beta 4$-spectrin, but it cannot cluster KCNQ3- and KCNQ4-subunit-containing potassium channels. In summary, both studies showed that the "quiverer" mice and human patients with mutations in $S P N B$ have similar phenotypes, which makes the "quiverer" mouse model a good model for this human syndromic auditory neuropathy.

Nodes of Ranvier are continuously restabilized and destabilized in mice with Spnb mutations, similar to demyelinating diseases where the myelin sheet is continuously degraded and remyelinated. Severe demyalinating disease can also cause hearing impairments (ElBadry etal. 2007) and sudden hearing loss has even been reported as an uncommon manifestation of multiple sclerosis (Daugherty et al. 1983; Schweitzer and Shepard 1989; Drulović et al. 1993). Another similarity lies in the fact, that as long as there is no axonal damage, nodes or myelin sheets can be reformed, but as soon as the axons take damage, they cannot be repaired (Saifetiarova et al. 2018).

In our study we were interested on how the delay of spiking or a subsequent loss of axons would affect sound encoding the ICC. Therefore we used quiverer-3J mice, a spectrin mutation leading to rather mild hearing impairments. In this variant compared to for instance the quiverer-4J mice, which are strongly hearing impaired, the ankyrin-G binding domain is not deleted (Parkinson et al. 2001). It has been shown, that the Spnb $b^{q v-3 J / q v-3 J}$ mutation leads to a reduced number of sodium channels in AIS. Action potentials are produced in normal numbers, but with impaired temporal precision (Lazarov et al. 2017).

In the rather young mice we used at the age of 6-12 weeks, axonal damage is probably limited, but velocity of action potential propagation was reduced. We were interested how much this mutation would affect sound encoding in the IC. Interestingly, the delay of spiking had only mild effects on spike rates. Onset spike rates were only slightly reduced, frequency and intensity coding as well as forward masking were normal. Phase-locking to the envelope of sAM tones was impaired at the higher limit of what is possible in ICNs $(64-128 \mathrm{~Hz})$. Temporal precision with reduced and delayed onset coding, which was dependent on the stimulation frequency, resulting in a greater delay and strong increase in jitter with faster stimulation. In summary, $S P N B^{q v-3 J / q v 3 J}$ patients would suffer most from a poor onset coding with increased jitter, especially and worsening at faster stimulation, also impacting the phase-locking abilities. 


\subsubsection{Diagnosis and treatment}

Auditory neuropathies or synaptopathies can be diagnosed by acquiring audiograms and measuring ABRs and OAEs. In contrast to hearing impairments with defects in cochlear amplification, OAEs are normal in auditory neuropathies while ABRs are absent or have low amplitudes.

Patients with auditory synaptopathies or neuropathies typically do not benefit much from conventional hearing aids but may benefit from cochlear implants provided that the electrical excitability of SGNs and auditory brainstem function are normal (ShehataDieler et al. 2007; Santarelli et al. 2013). However, benefits from cochlear implants for neural forms of auditory neuropathy vary a lot (Berlin et al. 2010).

As the cochlear implant bypasses the IHCs and directly stimulates the SGNs, the exact cause of the auditory neuropathy can have great influence on whether a cochlear implant will be beneficial (Eppsteiner et al. 2012). Good performers have mutations in the IHCs while poor performance is associated with mutations in SGN expressed genes. For instance individuals with $O T O F$-related deafness have been reported to benefit a lot from cochlear implants (Rouillon etal. 2006; Wu et al. 2011), as the mutation is located in the IHCs. On the other hand, in children with cochlear nerve deficiency, which have a similar hearing function as those with OTOF mutations, cochlear implants are obviously contraindicated (Buchman et al. 2006).

To know beforehand if a cochlear implant could be beneficial for a patient, additional clinical testing is advised. Pure tone audiograms, routinely used in clinical diagnosis of hearing impairments, do not suffice to identify auditory neuropathies in general. Determining speech comprehension is not specific enough to identify auditory neuropathies. However, measuring cochlear potentials may help to identify pre- and post-synaptic disorders of IHCs or SGNs (Santarelli et al. 2008). In detail, a ratio between cochlear SPs and auditory nerve action potentials may be useful to identify these disorders, as well as "hidden hearing loss", for example age-related or noise-induced loss of cochlear nerve synapses that lead to hearing deficits in difficult listening situations, despite the presence of normal thresholds in pure tone audiograms (Liberman et al. 2016).

Determining speech comprehension in noisy background helps diagnosing auditory neuropathies, when audiograms are relatively normal. However, the best clinical hearing test to identify an auditory neuropathy or synaptopathy would need to be independent of language and not influenced by cochlear amplification. We suggest for identifying patients with an $O T O F^{I 515 T / I 515 T}$ mutation or another synaptopathy with impaired vesicle replenishment, a simple gap detection test is likely the best psychoacoustic measure. It should yield normal results in cochlear amplifier defects, so it might also help to diagnose mixed forms with auditory neuropathy and impaired cochlear amplification. The procedure and setup are straightforward and easy to implement. Based on our results, designing a clinical test to identify $S P N B^{q v-3 J / q v-3 J}$ mutations might include a clinical task, where patients have to discriminate a pure tone from an sAM tone at $128 \mathrm{~Hz}$ and at $8 \mathrm{~Hz}$ as a control. However, neither of these tests are yet commonly used in clinics. 
For those patients with residual hearing that do not profit from conventional hearing aids it would be helpful to be able to improve the residual hearing when cochlear implantation is contraindicated by pre-processing sounds according to the needs of the patients to enhance as much of the residual hearing function as possible. Our results suggest that that possible treatment strategies might include hearing aids that do not amplify all sounds but reduce the input sounds into the ear. OTOF ${ }^{I 515 T / I 515 T}$ patients had a strong adaptation to continuous sounds and could benefit from hearing aids with speech processors that would reduce the sounds to the most important features needed for speech perception and mute all background noises and sound features that are not absolutely necessary to understand speech. The timing deficit and rate-dependency of which patients with a $S P N B^{q v-3 J / q v-3 J}$ mutation suffer is less likely to be treated with hearing aids, as speech can hardly be made "slower". However, enhancing the speech to noise ratio through hearing aids might be beneficial for many patients with auditory neuropathy, including those with a $S P N B^{q v-3 J / q v-3 J}$ mutation. This could be achieved by using noise-canceling headphones combined with directional microphones or wireless microphone signals that are also used for cochlear implant users, to get an input signal which only consists of the sound trace the patient is interested in without background noise.

\subsection{Summary and Conclusion}

Auditory coding in the ICC is very diverse and can be affected by different mutations. Mouse models for auditory synaptopathy and neuropathy are a useful tool to study the disease mechanisms of human patients with hearing disorders. Furthermore, this approach can be used to study the effects of altered sound encoding on the ICC to get insights into its function.

In this study we could show that tonotopy in the ICC is unaffected in mouse models with an intact cochlear and normal frequency coding in the SGNs. Moreover, it seems like thresholds are as well coded mainly by cochlear deficits while spike rates can be partially compensated in the auditory brainstem. However, this is only possible for mutations that cause moderate hearing impairments and not possible anymore if the defect is as severe as the Otof ${ }^{P g a / P g a}$ mutation, where frequency coding in the ICNs was disrupted, thresholds were elevated, and the extremely low spike rates could not be compensated at all. For defects which affect spike timing it likely depends on the affected time frame and the nature of the defect if compensation is possible. When replenishment is impaired, compensation seems not to be possible. For an increased variance in first spike latency in SGN spiking, compensation might be possible, if it still lies in the integration window of the input receiving neuron. Furthermore, increased adaptation has no effect on precision of phase-locking in response to sAM tones but is reflected in an increased adaptation in ICNs for low modulation frequencies. In contrast, a rate dependent increase in jitter reduces the upper limit where phase-locking is still possible in the ICC.

In addition to that, we showed that auditory neuropathy is an important diagnosis to make, including the identification of the site where the defect is present. This information 
is needed to be able to select the correct treatment for a patient. Generally, patients diagnosed with auditory neuropathy with impaired sound processing, but normal cochlear amplification do not benefit from conventional hearing aids, which amplify all sounds, but would need cochlear implants or hearing aids that reduce the sound inputs. To know where the exact defect is helps a lot to predict whether a cochlear implantation might be beneficial. 



\section{A. References}

Adams, J. C. (1979). "Ascending Projections to the Inferior Colliculus". J. Comp. Neurol. 183(3): $519-538$.

Aitkin, L. (1991). "Rate-Level Functions of Neurons in the Inferior Colliculus of Cats Measured with the Use of Free-Field Sound Stimuli". J. Neurophysiol. 65(2): 383392.

Altrock, W. D., tom Dieck, S., Sokolov, M., Meyer, A. C., Sigler, A., Brakebusch, C., Fässler, R., Richter, K., Boeckers, T. M., Potschka, H., Brandt, C., Löscher, W., Grimberg, D., Dresbach, T., Hempelmann, A., Hassan, H., Balschun, D., Frey, J. U., Brandstätter, J. H., Garner, C. C., Rosenmund, C., and Gundelfinger, E. D. (2003). "Functional Inactivation of a Fraction of Excitatory Synapses in Mice Deficient for the Active Zone Protein Bassoon". Neuron. 37(5): 787-800.

Anderson, L. A. and Malmierca, M. S. (2012). "The Effect of Auditory Cortex Deactivation on Stimulus-Specific Adaptation in the Inferior Colliculus of the Rat". Eur. J. Neurosci. 37(1): 52-62.

Ayala, Y. A., Udeh, A., Dutta, K., Bishop, D., Malmierca, M. S., and Oliver, D. L. (2015). "Differences in the Strength of Cortical and Brainstem Inputs to SSA and Non-SSA Neurons in the Inferior Colliculus". Sci. Rep. 5(10383): 1-17.

Ayala, Y. A. and Malmierca, M. S. (2013). "Stimulus-Specific Adaptation and Deviance Detection in the Inferior Colliculus". Front. Neural Circuits. 6(89): 1-16.

El-Badry, M. M., Ding, D.-l., McFadden, S. L., and Eddins, A. C. (2007). "Physiological Effects of Auditory Nerve Myelinopathy in Chinchillas". Eur. J. Neurosci. 25(5): $1437-1446$.

Batra, R. and Fitzpatrick, D. (2002). "Monaural and Binaural Processing in the Ventral Nucleus of the Lateral Lemniscus: A Major Source of Inhibition to the Inferior Colliculus". Hear. Res. 168(1-2): 90-97.

Berghs, S., Aggujaro, D., Dirkx, R., Maksimova, E., Stabach, P., Hermel, J.-M., Zhang, J.-P., Philbrick, W., Slepnev, V., Ort, T., and Solimena, M. (2000). " $\beta$ IV Spectrin, a New Spectrin Localized at Axon Initial Segments and Nodes of Ranvier in the Central and Peripheral Nervous System". J. Cell. Biol. 151(5): 985-1002.

Berlin, C. I., Hood, L. J., Morlet, T., Wilensky, D., Li, L., Mattingly, K. R., TaylorJeanfreau, J., Keats, B. J. B., John, P. S., Montgomery, E., Shallop, J. K., Russell, B. A., and Frisch, S. A. (2010). "Multi-Site Diagnosis and Management of 260 Patients with Auditory Neuropathy/Dys-Synchrony (Auditory Neuropathy Spectrum Disorder)". Int. J. Audiol. 49(1): 30-43.

Beyerl, B. D. (1978). "Afferent Projections to the Central Nucleus of the Inferior Colliculus in the Rat". Brain. Res. 145(2): 209-223.

Bourk, T. R. (1976). "Electrical Responses of Neural Units in the Anteroventral Cochlear Nucleus of the Cat." (PhD Thesis). 
Brand, A., Urban, R., and Grothe, B. (2000). "Duration Tuning in the Mouse Auditory Midbrain". J. Neurophysiol. 84(4): 1790-1799.

Brownell, W. E. (1990). "Outer Hair Cell Electromotility and Otoacoustic Emissions". Ear Hear. 11(2): 82-92.

Brunso-Bechtold, J. K., Thompson, G. C., and Masterton, R. B. (1981). "HRP Study of the Organization of Auditory Afferents Ascending to Central Nucleus of Inferior Colliculus in Cat". J. Comp. Neurol. 197(4): 705-722.

Buchman, C. A., Roush, P. A., Teagle, H. F. B., Brown, C. J., Zdanski, C. J., and Grose, J. H. (2006). "Auditory Neuropathy Characteristics in Children with Cochlear Nerve Deficiency". Ear Hear. 27(4): 399-408.

Buran, B. N., Strenzke, N., Neef, A., Gundelfinger, E. D., Moser, T., and Liberman, M. C. (2010). "Onset Coding Is Degraded in Auditory Nerve Fibers from Mutant Mice Lacking Synaptic Ribbons". J. Neurosci. 30(22): 7587-7597.

Cant, N. B. (2005). "Projections from the Cochlear Nuclear Complex to the Inferior Colliculus". In: Winer, J. A. and Schreiner, C. E. (Eds.) "The Inferior Colliculus": 115-131. Springer-Verlag New York, New York.

Casseday, J. H., Ehrlich, D., and Covey, E. (1994). "Neural Tuning for Sound Duration: Role of Inhibitory Mechanisms in the Inferior Colliculus". Science. 264(5160): 847850.

Chakrabarti, R., Michanski, S., and Wichmann, C. (2018). "Vesicle Sub-Pool Organization at Inner Hair Cell Ribbon Synapses". EMBO J. e44937.

Chambers, A. R., Resnik, J., Yuan, Y., Whitton, J. P., Edge, A. S., Liberman, M. C., and Polley, D. B. (2016). "Central Gain Restores Auditory Processing Following Near-Complete Cochlear Denervation". Neuron. 89(4): 867-879.

Chapochnikov, N. M., Takago, H., Huang, C.-H., Pangršič, T., Khimich, D., Neef, J., Auge, E., Göttfert, F., Hell, S. W., Wichmann, C., Wolf, F., and Moser, T. (2014). "Uniquantal Release through a Dynamic Fusion Pore Is a Candidate Mechanism of Hair Cell Exocytosis". Neuron. 17(83): 1389-1403.

Cheng, Y., Nakamura, M., Matsunaga, T., and Kaga, K. (2017). "A Case of Auditory Neuropathy Revealed by OTOF Gene Mutation Analysis in a Junior High School Girl". J. Otol. 12(4): 202-206.

Choi, B. Y., Ahmed, Z. M., Riazuddin, S., Bhinder, M. A., Shahzad, M., Husnain, T., Riazuddin, S., Griffith, A. J., and Friedman, T. B. (2009). "Identities and Frequencies of Mutations of the Otoferlin Gene (OTOF) Causing DFNB9 Deafness in Pakistan". Clin. Genet. 75(3): 237-243.

Collin, R. W. J., Kalay, E., Oostrik, J., Caylan, R., Wollnik, B., Arslan, S., den Hollander, A. I., Birinci, Y., Lichtner, P., Strom, T. M., Toraman, B., Hoefsloot, L. H., Cremers, C. W. R. J., Brunner, H. G., Cremers, F. P. M., Karaguzel, A., and Kremer, H. (2007). "Involvement of DFNB59 Mutations in Autosomal Recessive Nonsyndromic Hearing Impairment". Hum. Mutat. 28(7): 718-723.

Covey, E. and Casseday, J. H. (1991). "The Monaural Nuclei of the Lateral Lemniscus in an Echolocating Bat: Parallel Pathways for Analyzing Temporal Features of Sound". J. Neurosci. 11(11): 3456-3470.

Cowper-Smith, C. D., Dingle, R. N., Guo, Y., Burkard, R., and Phillips, D. P. (2010). "Synchronous Auditory Nerve Activity in the Carboplatin-Chinchilla Model of Auditory Neuropathy". J. Acoust. Soc. Am. 128(1): EL56-EL62. 
Daugherty, W. T., Lederman, R. J., Nodar, R. H., and Conomy, J. P. (1983). "Hearing Loss in Multiple Sclerosis". Arch. Neurol. 40(1): 33-35.

Delmaghani, S., del Castillo, F. J., Michel, V., Leibovici, M., Aghaie, A., Ron, U., Van Laer, L., Ben-Tal, N., Van Camp, G., Weil, D., Langa, F., Lathrop, M., Avan, P., and Petit, C. (2006). "Mutations in the Gene Encoding Pejvakin, a Newly Identified Protein of the Afferent Auditory Pathway, Cause DFNB59 Auditory Neuropathy". Nat. Genet. 38(7): 770-778.

Drulović, B., Ribarić-Jankes, K., Kostić, V. S., and Šternić, N. (1993). "Sudden Hearing Loss as the Initial Monosymptom of Multiple Sclerosis". Neurology. 43(12): 2703.

Dulon, D., Safieddine, S., Jones, S. M., and Petit, C. (2009). "Otoferlin Is Critical for a Highly Sensitive and Linear Calcium-Dependent Exocytosis at Vestibular Hair Cell Ribbon Synapses". J. Neurosci. 29(34): 10474-10487.

Duncker, S. V., Franz, C., Kuhn, S., Schulte, U., Campanelli, D., Brandt, N., Hirt, B., Fakler, B., Blin, N., Ruth, P., Engel, J., Marcotti, W., Zimmermann, U., and Knipper, M. (2013). "Otoferlin Couples to Clathrin-Mediated Endocytosis in Mature Cochlear Inner Hair Cells". J. Neurosci. 33(22): 9508-9519.

Duque, D., Pais, R., and Malmierca, M. S. (2018). "Stimulus-Specific Adaptation in the Anesthetized Mouse Revealed by Brainstem Auditory Evoked Potentials". Hear. Res. in press.

Egorova, M., Ehret, G., Vartanian, I., and Esser, K. H. (2001). "Frequency Response Areas of Neurons in the Mouse Inferior Colliculus. I. Threshold and Tuning Characteristics". Exp. Brain. Res. 140(2): 145-161.

Egorova, M., Vartanyan, I., and Ehret, G. (2006). "Frequency Response Areas of Mouse Inferior Colliculus Neurons: II. Critical Bands". Neuroreport. 17(17): 1783-1786.

Ehret, G. and Merzenich, M. M. (1988a). "Neuronal Discharge Rate Is Unsuitable for Encoding Sound Intensity at the Inferior-Colliculus Level". Hear. Res. 35(1): 1-7.

Ehret, G. and Merzenich, M. M. (1988b). "Complex Sound Analysis (Frequency Resolution, Filtering and Spectral Integration) by Single Units of the Inferior Colliculus of the Cat". Brain Res. 13(2): 139-163.

Ehret, G. and Moffat, A. J. (1985). "Inferior Colliculus of the House Mouse". J. Comp. Physiol. A. 156(5): 619-635.

Eppsteiner, R. W., Shearer, A. E., Hildebrand, M. S., DeLuca, A. P., Ji, H., Dunn, C. C., Black-Ziegelbein, E. A., Casavant, T. L., Braun, T. A., Scheetz, T. E., Scherer, S. E., Hansen, M. R., Gantz, B. J., and Smith, R. J. (2012). "Prediction of Cochlear Implant Performance by Genetic Mutation: The Spiral Ganglion Hypothesis". Hear. Res. 292(1-2): 51-58.

Fay, R. R. (1994). "Comparative Auditory Research". In: Fay, R. R. and Popper, A. N. (Eds.) "Comparative Hearing: Mammals": 1-17. Springer-Verlag New York, New York.

Fejtova, A. and Gundelfinger, E. D. (2006). "Molecular Organization and Assembly of the Presynaptic Active Zone of Neurotransmitter Release". Results Probl. Cell. Differ. 43: $49-68$.

Fettiplace, R. and Hackney, C. M. (2006). "The Sensory and Motor Roles of Auditory Hair Cells". Nat. Rev. Neurosci. 7(1): 19-29.

Fitzpatrick, D. C., Batra, R., Stanford, T. R., and Kuwada, S. (1997). "A Neuronal Population Code for Sound Localization". Nature. 388: 871-874. 
Friauf, E. and Ostwald, J. (1988). "Divergent Projections of Physiologically Characterized Rat Ventral Cochlear Nucleus Neurons as Shown by Intra-Axonal Injection of Horseradish Peroxidase". Exp. Brain. Res. 73(2): 263-284.

Frisina, R. D., Smith, R. L., and Chamberlain, S. C. (1990). "Encoding of Amplitude Modulation in the Gerbil Cochlear Nucleus: I. A Hierarchy of Enhancement". Hear. Res. 44(2-3): 99-122.

Frohman, E. M., Racke, M. K., and Raine, C. S. (2006). "Multiple Sclerosis - The Plaque and Its Pathogenesis". N. Engl. J. Med. 354(9): 942-955.

Galambos, R. and Davis, H. (1943). "The Response of Single Auditory-Nerve Fibers to Acoustic Stimulation". J. Neurophysiol. 6(1): 39-57.

Glowatzki, E. and Fuchs, P. A. (2002). "Transmitter Release at the Hair Cell Ribbon Synapse". Nat. Neurosci. 5(2): 147-154.

Goldberg, J. M. and Brown, P. B. (1969). "Response of Binaural Neurons of Dog Superior Olivary Complex to Dichotic Tonal Stimuli: Some Physiological Mechanisms of Sound Localization". J. Neurophysiol. 32(4): 613-636.

Golding, N. L., Robertson, D., and Oertel, D. (1995). "Recordings from Slices Indicate That Octopus Cells of the Cochlear Nucleus Detect Coincident Firing of Auditory Nerve Fibers with Temporal Precision". J. Neurosci. 15(4): 3138-3153.

Goutman, J. D. and Glowatzki, E. (2007). "Time Course and Calcium Dependence of Transmitter Release at a Single Ribbon Synapse". Proc. Natl. Acad. Sci. U S A. 104(41): 16341-16346.

Grant, L., Yi, E., and Glowatzki, E. (2010). "Two Modes of Release Shape the Postsynaptic Response at the Inner Hair Cell Ribbon Synapse". J. Neurosci. 30(12): 4210-4220.

Greenwood, D. D. (1996). "Comparing Octaves, Frequency Ranges, and Cochlear-Map Curvature across Species". Hear. Res. 94(1-2): 157-162.

Grothe, B., Pecka, M., and McAlpine, D. (2010). "Mechanisms of Sound Localization in Mammals". Physiol. Rev. 90(3): 983-1012.

Gundelfinger, E. D., Reissner, C., and Garner, C. C. (2015). "Role of Bassoon and Piccolo in Assembly and Molecular Organization of the Active Zone". Front. Synaptic Neurosci. 7(19): 1-11.

Harris, D. M. and Dallos, P. (1979). "Forward Masking of Auditory Nerve Fiber Responses". J. Neurophysiol. 42(4): 1083-1107.

Harrison, R. V. (1998). "An Animal Model of Auditory Neuropathy". Ear Hear. 19(5): 355-361.

Heffner, H. E. and Heffner, R. S. (2007). "Hearing Ranges of Laboratory Animals". J. Am. Assoc. Lab. Anim. Sci. 46(1): 20-22.

Heil, P. (1998). "Neuronal Coding of Interaural Transient Envelope Disparities". Eur. J. Neurosci. 10(9): 2831-2847.

Heil, P. and Neubauer, H. (2001). "Temporal Integration of Sound Pressure Determines Thresholds of Auditory-Nerve Fibers". J. Neurosci. 21(18): 7404-7415.

Heil, P., Neubauer, H., Irvine, D. R., and Brown, M. (2007). "Spontaneous Activity of Auditory-Nerve Fibers: Insights into Stochastic Processes at Ribbon Synapses". J. Neurosci. 27(31): 8457-8474.

Heil, P. and Peterson, A. J. (2015). "Basic Response Properties of Auditory Nerve Fibers: A Review". Cell Tissue Res. 361(1): 129-158. 
Heil, P. and Peterson, A. J. (2017). "Spike Timing in Auditory-nerve Fibers during Spontaneous Activity and Phase Locking". Synapse. 71(1): 5-36.

Hossain, W. A., Antic, S. D., Yang, Y., Rasband, M. N., and Morest, D. K. (2005). "Where Is the Spike Generator of the Cochlear Nerve? Voltage-Gated Sodium Channels in the Mouse Cochlea". J. Neurosci. 25(29): 6857-6868.

Huang, C. Y.-M. and Rasband, M. N. (2018). "Axon Initial Segments: Structure, Function, and Disease". Ann. N. Y. Acad. Sci. 1420(1): 46-61.

Itoh, K., Kaneko, T., Kudo, M., and Mizuno, N. (1984). "The Intercollicular Region in the Cat: A Possible Relay in the Parallel Somatosensory Pathways from the Dorsal Column Nuclei to the Posterior Complex of the Thalamus". Brain Res. 308(1): 166171.

Jeffress, L. A. (1948). "A Place Theory of Sound Localization". J. Comp. Physiol. Psychol. 41(1): 35-39.

Jenkins, S. M. and Bennett, V. (2001). "Ankyrin-G Coordinates Assembly of the SpectrinBased Membrane Skeleton, Voltage-Gated Sodium Channels, and L1 CAMs at Purkinje Neuron Initial Segments". J. Cell Biol. 155(5): 739-746.

Jing, Z., Rutherford, M. A., Takago, H., Frank, T., Fejtova, A., Khimich, D., Moser, T., and Strenzke, N. (2013). "Disruption of the Presynaptic Cytomatrix Protein Bassoon Degrades Ribbon Anchorage, Multiquantal Release, and Sound Encoding at the Hair Cell Afferent Synapse". J. Neurosci. 33(10): 4456-4467.

Johnson, C. P. and Chapman, E. R. (2010). "Otoferlin Is a Calcium Sensor That Directly Regulates SNARE-Mediated Membrane Fusion". J. Cell Biol. 191(1): 187-197.

Joris, P. X., Carney, L. H., Smith, P. H., and Yin, T. C. (1994). "Enhancement of Neural Synchronization in the Anteroventral Cochlear Nucleus. I. Responses to Tones at the Characteristic Frequency". J. Neurophysiol. 71(3): 1022-1036.

Joris, P. X. and Yin, T. C. T. (1992). "Responses to Amplitude-modulated Tones in the Auditory Nerve of the Cat". J. Acoust. Soc. Am. 91(1): 215-232.

Joris, P., Schreiner, C., and Rees, A. (2004). "Neural Processing of Amplitude-Modulated Sounds". Physiol. Rev. 84(2): 541-577.

Jung, S., Maritzen, T., Wichmann, C., Jing, Z., Neef, A., Revelo, N. H., Al-Moyed, H., Meese, S., Wojcik, S. M., Panou, I., Bulut, H., Schu, P., Ficner, R., Reisinger, E., Rizzoli, S. O., Neef, J., Strenzke, N., Haucke, V., and Moser, T. (2015). "Disruption of Adaptor Protein $2 \mu$ (AP-2 $\mu$ ) in Cochlear Hair Cells Impairs Vesicle Reloading of Synaptic Release Sites and Hearing". EMBO J. 34(21): 2686-2702.

Kandel, E. R., Schwartz, J. H., and Jessell, T. (2012). "Principles of Neural Science". McGraw-Hill Medical, New York.

Khanna, S. M. and Teich, M. C. (1989). "Spectral Characteristics of the Responses of Primary Auditory-Nerve Fibers to Amplitude-Modulated Signals". Hear. Res. 39(12): $143-157$.

Khimich, D., Nouvian, R., Pujol, R., tom Dieck, S., Egner, A., Gundelfinger, E. D., and Moser, T. (2005). "Hair Cell Synaptic Ribbons Are Essential for Synchronous Auditory Signalling". Nature. 434(7035): 889-894.

Klug, A., Khan, A., Burger, R. M., Bauer, E. E., Hurley, L. M., Yang, L., Grothe, B., Halvorsen, M. B., and Park, T. J. (2000). "Latency as a Function of Intensity in Auditory Neurons: Influences of Central Processing". Hear. Res. 148(1-2): 107-123. 
Knierim, E., Gill, E., Seifert, F., Morales-Gonzalez, S., Unudurthi, S. D., Hund, T. J., Stenzel, W., and Schuelke, M. (2017). "A Recessive Mutation in Beta-IV-Spectrin (SPTBN4) Associates with Congenital Myopathy, Neuropathy, and Central Deafness". Hum. Genet. 136(7): 903-910.

Kole, M. H. P., Ilschner, S. U., Kampa, B. M., Williams, S. R., Ruben, P. C., and Stuart, G. J. (2008). "Action Potential Generation Requires a High Sodium Channel Density in the Axon Initial Segment". Nat. Neurosci. 11(2): 178-186.

Komada, M. and Soriano, P. (2002). " $\beta$ IV-Spectrin Regulates Sodium Channel Clustering through Ankyrin-G at Axon Initial Segments and Nodes of Ranvier". J. Cell Biol. 156(2): $337-348$.

Kopp-Scheinpflug, C. and Tempel, B. L. (2015). "Decreased Temporal Precision of Neuronal Signaling as a Candidate Mechanism of Auditory Processing Disorder". Hear. Res. 330: 213-220.

Krächan, E. G., Fischer, A. U., Franke, J., and Friauf, E. (2016). "Synaptic Reliability and Temporal Precision Are Achieved via High Quantal Content and Effective Replenishment: Auditory Brainstem versus Hippocampus". J. Physiol. 595(3): 839864.

Krebs, B., Lesica, N. A., and Grothe, B. (2008). "The Representation of Amplitude Modulations in the Mammalian Auditory Midbrain." J. Neurophysiol. 100(3): 16021609.

Lacas-Gervais, S., Guo, J., Strenzke, N., Scarfone, E., Kolpe, M., Jahkel, M., De Camilli, P., Moser, T., Rasband, M. N., and Solimena, M. (2004). " $\beta$ IV $\Sigma 1$ Spectrin Stabilizes the Nodes of Ranvier and Axon Initial Segments". J. Cell Biol. 166(7): 983-990.

Langner, G. (1992). "Periodicity Coding in the Auditory System". Hear. Res. 60(2): $115-142$.

Langner, G., Albert, M., and Briede, T. (2002). "Temporal and Spatial Coding of Periodicity Information in the Inferior Colliculus of Awake Chinchilla (Chinchilla Laniger)". Hear. Res. 168(1): 110-130.

Lazarov, E., Dannemeyer, M., Feulner, B., Enderlein, J., Gutnick, M. J., Wolf, F., and Neef, A. (2017). "An Axon Initial Segment Is Required for Temporal Precision in Action Potential Encoding by Neuronal Populations". submitted. Available from: URL: https://arxiv.org/abs/1711.03383.

Liberman, M. C. (1978). "Auditory-Nerve Response from Cats Raised in a Low-Noise Chamber". J. Acoust. Soc. Am. 63(2): 442-455.

Liberman, M. C. (1982a). "The Cochlear Frequency Map for the Cat: Labeling AuditoryNerve Fibers of Known Characteristic Frequency". J. Acoust. Soc. Am. 72(5): 14411449.

Liberman, M. C. (1991). "Central Projections of Auditory-Nerve Fibers of Differing Spontaneous Rate. I. Anteroventral Cochlear Nucleus". J. Comp. Neurol. 313(2): 240-258.

Liberman, M. C., Epstein, M. J., Cleveland, S. S., Wang, H., and Maison, S. F. (2016). "Toward a Differential Diagnosis of Hidden Hearing Loss in Humans". PLoS ONE. 11(9): e0162726.

Liberman, M. C., Gao, J., He, D. Z. Z., Wu, X., Jia, S., and Zuo, J. (2002). "Prestin Is Required for Electromotility of the Outer Hair Cell and for the Cochlear Amplifier". Nature. 419(6904): 300-304. 
Liberman, M. (1982b). "Single-Neuron Labeling in the Cat Auditory Nerve". Science. 216(4551): 1239-1241.

Linkert, M., Rueden, C. T., Allan, C., Burel, J.-M., Moore, W., Patterson, A., Loranger, B., Moore, J., Neves, C., MacDonald, D., Tarkowska, A., Sticco, C., Hill, E., Rossner, M., Eliceiri, K. W., and Swedlow, J. R. (2010). "Metadata Matters: Access to Image Data in the Real World". J. Cell Biol. 189(5): 777-782.

Malmierca, M. S., Cristaudo, S., Pérez-González, D., and Covey, E. (2009). "StimulusSpecific Adaptation in the Inferior Colliculus of the Anesthetized Rat". J. Neurosci. 29(17): 5483-5493.

Marlin, S., Feldmann, D., Nguyen, Y., Rouillon, I., Loundon, N., Jonard, L., Bonnet, C., Couderc, R., Garabedian, E. N., Petit, C., and Denoyelle, F. (2010). "TemperatureSensitive Auditory Neuropathy Associated with an Otoferlin Mutation: Deafening Fever!" Biochem. Biophys. Res. Commun. 394(3): 737-742.

Meddis, R. and O'Mard, L. P. (2005). "A Computer Model of the Auditory-Nerve Response to Forward-Masking Stimuli". J. Acoust. Soc. Am. 117(6): 3787-3798.

Meininger, V., Pol, D., and Derer, P. (1986). "The Inferior Colliculus of the Mouse. A Nissl and Golgi Study". Neuroscience. 17(4): 1159-1179.

Melcher, J. R., Guinan, J. J., Knudson, I. M., and Kiang, N. Y. (1996). "Generators of the Brainstem Auditory Evoked Potential in Cat II. Correlating Lesion Sites with Waveform Changes". Hear. Res. 93(1-2): 28-51.

Melcher, J. R. and Kiang, N. (1996). "Generators of the Brainstem Auditory Evoked Potential in Cat III: Identified Cell Populations". Hear. Res. 93(1-2): 52-71.

Melcher, J. R., Knudson, I. M., Fullerton, B. C., Guinan, J. J., Norris, B. E., and Kiang, N. (1996). "Generators of the Brainstem Auditory Evoked Potential in Cat. I. An Experimental Approach to Their Identification". Hear. Res. 93(1-2): 1-27.

Merchán, M., Aguilar, L. A., Lopez-Poveda, E. A., and Malmierca, M. S. (2005). "The Inferior Colliculus of the Rat: Quantitative Immunocytochemical Study of GABA and Glycine". Neuroscience. 136(3): 907-925.

Merchan-Perez, A. and Liberman, M. C. (1996). "Ultrastructural Differences among Afferent Synapses on Cochlear Hair Cells: Correlations with Spontaneous Discharge Rate". J. Comp. Neurol. 371(2): 208-221.

Merzenich, M. M. and Reid, M. D. (1974). "Representation of the Cochlea within the Inferior Colliculus of the Cat". Brain Res. 77(3): 397-415.

Meyer, A. C., Frank, T., Khimich, D., Hoch, G., Riedel, D., Chapochnikov, N. M., Yarin, Y. M., Harke, B., Hell, S. W., Egner, A., and Moser, T. (2009). "Tuning of Synapse Number, Structure and Function in the Cochlea". Nat. Neurosci. 12(4): 444-453.

Michalski, N., Goutman, J. D., Auclair, S. M., Monvel, J. B. de, Tertrais, M., Emptoz, A., Parrin, A., Nouaille, S., Guillon, M., Sachse, M., Ciric, D., Bahloul, A., Hardelin, J.-P., Sutton, R. B., Avan, P., Krishnakumar, S. S., Rothman, J. E., Dulon, D., Safieddine, S., and Petit, C. (2017). "Otoferlin Acts as a Ca2+ Sensor for Vesicle Fusion and Vesicle Pool Replenishment at Auditory Hair Cell Ribbon Synapses". Elife. 6: e31013.

Mirghomizadeh, F., Pfister, M., Apaydin, F., Petit, C., Kupka, S., Pusch, C. M., Zenner, H. P., and Blin, N. (2002). "Substitutions in the Conserved C2C Domain of Otoferlin Cause DFNB9, a Form of Nonsyndromic Autosomal Recessive Deafness". Neurobiol. Dis. 10(2): 157-164. 
Moore, B. C. J. (2008). "The Role of Temporal Fine Structure Processing in Pitch Perception, Masking, and Speech Perception for Normal-Hearing and Hearing-Impaired People". J. Assoc. Res. Otolaryngol. 9(4): 399-406.

Moore, D. R. (1991). "Anatomy and Physiology of Binaural Hearing". Audiology. 30(3): $125-134$.

Moser, T., Neef, A., and Khimich, D. (2006). "Mechanisms Underlying the Temporal Precision of Sound Coding at the Inner Hair Cell Ribbon Synapse". J. Physiol. 576(1): $55-62$.

Moser, T. and Beutner, D. (2000). "Kinetics of Exocytosis and Endocytosis at the Cochlear Inner Hair Cell Afferent Synapse of the Mouse". Proc. Natl. Acad. Sci. $U S$ A. 97(2): 883-888.

Moser, T., Predoehl, F., and Starr, A. (2013). "Review of Hair Cell Synapse Defects in Sensorineural Hearing Impairment". Otol. Neurotol. 34(6): 995-1004.

Moser, T. and Starr, A. (2016). "Auditory Neuropathy - Neural and Synaptic Mechanisms". Nat. Rev. Neurol. 12(3): 135-149.

Nelson, K. R., Gilmore, R. L., and Massey, A. (1988). "Acoustic Nerve Conduction Abnormalities in Guillain-Barré Syndrome". Neurology. 38(8): 1263.

Nouvian, R., Beutner, D., Parsons, T. D., and Moser, T. (2006). "Structure and Function of the Hair Cell Ribbon Synapse". J. Membr. Biol. 209(2-3): 153-165.

Nouvian, R., Neef, J., Bulankina, A. V., Reisinger, E., Pangršič, T., Frank, T., Sikorra, S., Brose, N., Binz, T., and Moser, T. (2011). "Exocytosis at the Hair Cell Ribbon Synapse Apparently Operates without Neuronal SNARE Proteins". Nat. Neurosci. 14(4): 411-413.

Oertel, D. (1999). "The Role of Timing in the Brain Stem Auditory Nuclei of Vertebrates". Annu. Rev. Physiol. 61(1): 497-519.

Oertel, D., Bal, R., Gardner, S. M., Smith, P. H., and Joris, P. X. (2000). "Detection of Synchrony in the Activity of Auditory Nerve Fibers by Octopus Cells of the Mammalian Cochlear Nucleus". Proc. Natl. Acad. Sci. U S A. 97(22): 11773-11779.

Oertel, D. and Wickesberg, R. E. (2002). "Ascending Pathways Through Ventral Nuclei of the Lateral Lemniscus and Their Possible Role in Pattern Recognition in Natural Sounds". In: Oertel, D., Fay, R. R., and Popper, A. N. (Eds.) "Integrative Functions in the Mammalian Auditory Pathway": 207-237. Springer-Verlag New York, New York.

Oertel, D., Wright, S., Cao, X.-J., Ferragamo, M., and Bal, R. (2011). "The Multiple Functions of T Stellate/Multipolar/Chopper Cells in the Ventral Cochlear Nucleus". Hear. Res. 276(1-2): 61-69.

Oliver, D. L. and Morest, D. K. (1984). "The Central Nucleus of the Inferior Colliculus in the Cat". J. Comp. Neurol. 222(2): 237-264.

Oliver, D. L., Kuwada, S., Yin, T. C., Haberly, L. B., and Henkel, C. K. (1991). "Dendritic and Axonal Morphology of HRP-injected Neurons in the Inferior Colliculus of the Cat". J. Comp. Neurol. 303(1): 75-100.

Oliver, D. L., Winer, J. A., Beckius, G. E., and Marie, R. L. S. (1994). "Morphology of GABAergic Neurons in the Inferior Colliculus of the Cat". J. Comp. Neurol. 340(1): 27-42. 
Ono, M., Bishop, D. C., and Oliver, D. L. (2017). "Identified GABAergic and Glutamatergic Neurons in the Mouse Inferior Colliculus Share Similar Response Properties." J. Neurosci. $0745-17$.

Palmer, A. R. and Russell, I. J. (1986). "Phase-Locking in the Cochlear Nerve of the Guinea-Pig and Its Relation to the Receptor Potential of Inner Hair-Cells". Hear. Res. 24(1): 1-15.

Palmer, A. R., Shackleton, T. M., Sumner, C. J., Zobay, O., and Rees, A. (2013). "Classification of Frequency Response Areas in the Inferior Colliculus Reveals Continua Not Discrete Classes: Inferior Colliculus Response Areas". J. Physiol. 591(16): 40034025.

Pangrsic, T., Lasarow, L., Reuter, K., Takago, H., Schwander, M., Riedel, D., Frank, T., Tarantino, L. M., Bailey, J. S., Strenzke, N., Brose, N., Müller, U., Reisinger, E., and Moser, T. (2010). "Hearing Requires Otoferlin-Dependent Efficient Replenishment of Synaptic Vesicles in Hair Cells". Nat. Neurosci. 13(7): 869-876.

Pangršič, T., Reisinger, E., and Moser, T. (2012). "Otoferlin: A Multi-C2 Domain Protein Essential for Hearing". Trends Neurosci. 35(11): 671-680.

Park, T. J., Klug, A., Holinstat, M., and Grothe, B. (2004). "Interaural Level Difference Processing in the Lateral Superior Olive and the Inferior Colliculus". J. Neurophysiol. 92(1): 289-301.

Parkinson, N. J., Olsson, C. L., Hallows, J. L., McKee-Johnson, J., Keogh, B. P., NobenTrauth, K., Kujawa, S. G., and Tempel, B. L. (2001). "Mutant $\beta$-Spectrin 4 Causes Auditory and Motor Neuropathies in Quivering Mice". Nat. Genet. 29(1): 61-65.

Patuzzi, R. B., Yates, G. K., and Johnstone, B. M. (1989). "Outer Hair Cell Receptor Current and Sensorineural Hearing Loss". Hear. Res. 42(1): 47-72.

Pennacchio, L. A. (2003). "Insights from Human/Mouse Genome Comparisons". Mamm. Genome. 14(7): 429-436.

Peruzzi, D., Bartlett, E., Smith, P. H., and Oliver, D. L. (1997). "A Monosynaptic GABAergic Input from the Inferior Colliculus to the Medial Geniculate Body in Rat". J. Neurosci. 17(10): 3766-3777.

Peruzzi, D., Sivaramakrishnan, S., and Oliver, D. L. (2000). "Identification of Cell Types in Brain Slices of the Inferior Colliculus". Neuroscience. 101(2): 403-416.

Preibisch, S., Saalfeld, S., and Tomancak, P. (2009). "Globally Optimal Stitching of Tiled 3D Microscopic Image Acquisitions". Bioinformatics. 25(11): 1463-1465.

Ramachandran, R., Davis, K. A., and May, B. J. (1999). "Single-Unit Responses in the Inferior Colliculus of Decerebrate Cats I. Classification Based on Frequency Response Maps". J. Neurophysiol. 82(1): 152-163.

Rance, G., Fava, R., Baldock, H., Chong, A., Barker, E., Corben, L., and Delatycki, M. B. (2008). "Speech Perception Ability in Individuals with Friedreich Ataxia". Brain. 131: 2002-2012.

Rance, G., Ryan, M. M., Bayliss, K., Gill, K., O'Sullivan, C., and Whitechurch, M. (2012). "Auditory Function in Children with Charcot-Marie-Tooth Disease". Brain. 135(5): $1412-1422$.

Rauch, S. (1987). "Hereditary deafness of central origin in two new alleles of the quivering mutation". Mouse News Lett. 78: 44. 
Rauschecker, J. and Tian, B. (2000). "Mechanisms and Streams for Processing of 'what' and 'Where' in Auditory Cortex". Proc. Natl. Acad. Sci. U.S.A. 97(22): 1180011806.

Reisinger, E., Bresee, C., Neef, J., Nair, R., Reuter, K., Bulankina, A., Nouvian, R., Koch, M., Bückers, J., Kastrup, L., Roux, I., Petit, C., Hell, S. W., Brose, N., Rhee, J.-S., Kügler, S., Brigande, J. V., and Moser, T. (2011). "Probing the Functional Equivalence of Otoferlin and Synaptotagmin 1 in Exocytosis". J. Neurosci. 31(13): 4886-4895.

Rhode, W. S., Oertel, D., and Smith, P. H. (1983). "Physiological Response Properties of Cells Labeled Intracellularly with Horseradish Peroxidase in Cat Ventral Cochlear Nucleus". J. Comp. Neurol. 213(4): 448-463.

Rhode, W. S. and Smith, P. H. (1986). "Encoding Timing and Intensity in the Ventral Cochlear Nucleus of the Cat". J. Neurophysiol. 56(2): 261-286.

Rhode, W. (2008). "Response Patterns to Sound Associated with Labeled Globular/Bushy Cells in Cat". Neuroscience. 154(1): 87-98.

Rodríguez-Ballesteros, M., del Castillo, F. J., Martín, Y., Moreno-Pelayo, M. A., Morera, C., Prieto, F., Marco, J., Morant, A., Gallo-Terán, J., Morales-Angulo, C., Navas, C., Trinidad, G., Tapia, M. C., Moreno, F., and del Castillo, I. (2003). "Auditory Neuropathy in Patients Carrying Mutations in the Otoferlin Gene (OTOF)". Hum. Mutat. 22(6): 451-456.

Rodríguez-Ballesteros, M., Reynoso, R., Olarte, M., Villamar, M., Morera, C., Santarelli, R., Arslan, E., Medá, C., Curet, C., Völter, C., Sainz-Quevedo, M., Castorina, P., Ambrosetti, U., Berrettini, S., Frei, K., Tedín, S., Smith, J., Cruz Tapia, M., Cavallé, L., Gelvez, N., Primignani, P., Gómez-Rosas, E., Martín, M., Moreno-Pelayo, M. A., Tamayo, M., Moreno-Barral, J., Moreno, F., and del Castillo, I. (2008). "A Multicenter Study on the Prevalence and Spectrum of Mutations in the Otoferlin Gene (OTOF) in Subjects with Nonsyndromic Hearing Impairment and Auditory Neuropathy". Hum. Mutat. 29(6): 823-831.

Rouillon, I., Marcolla, A., Roux, I., Marlin, S., Feldmann, D., Couderc, R., Jonard, L., Petit, C., Denoyelle, F., Garabédian, E. N., and Loundon, N. (2006). "Results of Cochlear Implantation in Two Children with Mutations in the OTOF Gene". Int. J. Pediatr. Otorhinolaryngol. 70(4): 689-696.

Roux, I., Safieddine, S., Nouvian, R., Grati, M., Simmler, M.-C., Bahloul, A., Perfettini, I., Le Gall, M., Rostaing, P., Hamard, G., Triller, A., Avan, P., Moser, T., and Petit, C. (2006). "Otoferlin, Defective in a Human Deafness Form, Is Essential for Exocytosis at the Auditory Ribbon Synapse". Cell. 127(2): 277-289.

Rueden, C. T., Schindelin, J., Hiner, M. C., DeZonia, B. E., Walter, A. E., Arena, E. T., and Eliceiri, K. W. (2017). "ImageJ2: ImageJ for the next Generation of Scientific Image Data". BMC Bioinformatics. 18(1): 529.

Ruel, J., Emery, S., Nouvian, R., Bersot, T., Amilhon, B., Van Rybroek, J. M., Rebillard, G., Lenoir, M., Eybalin, M., Delprat, B., Sivakumaran, T. A., Giros, B., El Mestikawy, S., Moser, T., Smith, R. J., Lesperance, M. M., and Puel, J.-L. (2008). "Impairment of SLC17A8 Encoding Vesicular Glutamate Transporter-3, VGLUT3, Underlies Nonsyndromic Deafness DFNA25 and Inner Hair Cell Dysfunction in Null Mice". Am. J. Hum. Genet. 83(2): 278-292. 
Rutherford, M. A., Chapochnikov, N. M., and Moser, T. (2012). "Spike Encoding of Neurotransmitter Release Timing by Spiral Ganglion Neurons of the Cochlea". J. Neurosci. 32(14): 4773-4789.

Ryan, A. and Miller, J. (1978). "Single Unit Responses in the Inferior Colliculus of the Awake and Performing Rhesus Monkey". Exp. Brain. Res. 32(3): 389-407.

Ryugo, D. K. and Sento, S. (1991). "Synaptic Connections of the Auditory Nerve in Cats: Relationship between Endbulbs of Held and Spherical Bushy Cells". J. Comp. Neurol. 305(1): 35-48.

Ryugo, D., Willard, F., and Fekete, D. (1981). "Differential Afferent Projections to the Inferior Colliculus from the Cochlear Nucleus in the Albino Mouse". Brain Res. 210(1-2): 342-349.

Sachs, M. B. and Abbas, P. J. (1974). "Rate versus Level Functions for Auditory-Nerve Fibers in Cats: Tone-Burst Stimuli". J. Acoust. Soc. Am. 56(6): 1835-1847.

Safieddine, S. and Wenthold, R. J. (1999). "SNARE Complex at the Ribbon Synapses of Cochlear Hair Cells: Analysis of Synaptic Vesicle- and Synaptic Membrane-Associated Proteins". Eur. J. Neurosci. 11(3): 803-812.

Saifetiarova, J., Shi, Q., Paukert, M., Komada, M., and Bhat, M. A. (2018). "Reorganization of Destabilized Nodes of Ranvier in $\beta$ IV Spectrin Mutants Uncovers Critical Timelines for Nodal Restoration and Prevention of Motor Paresis". J. Neurosci. 28(38): $6267-6282$.

Saldaña, E. and Merchán, M. A. (2005). "Intrinsic and Commissural Connections of the Inferior Colliculus". In: Winer, J. A. and Schreiner, C. E. (Eds.) "The Inferior Colliculus": 155-181. Springer-Verlag New York, New York.

Salvi, R. J., Wang, J., Ding, D., Stecker, N., and Arnold, S. (1999). "Auditory Deprivation of the Central Auditory System Resulting from Selective Inner Hair Cell Loss: Animal Model of Auditory Neuropathy". Scand. Audiol. Suppl. 51: 1-12.

Santarelli, R., Del Castillo, I., Cama, E., Scimemi, P., and Starr, A. (2015). "Audibility, Speech Perception and Processing of Temporal Cues in Ribbon Synaptic Disorders Due to OTOF Mutations". Hear. Res. 330: 200-212.

Santarelli, R., Del Castillo, I., Rodríguez-Ballesteros, M., Scimemi, P., Cama, E., Arslan, E., and Starr, A. (2009). "Abnormal Cochlear Potentials from Deaf Patients with Mutations in the Otoferlin Gene". J. Assoc. Res. Otolaryngol. 10(4): 545-556.

Santarelli, R., Rossi, R., and Arslan, E. (2013). "Assistive Devices for Patients with Auditory Neuropathy: Hearing Aid Use". Semin. Hear. 34(01): 51-64.

Santarelli, R., Starr, A., Michalewski, H. J., and Arslan, E. (2008). "Neural and Receptor Cochlear Potentials Obtained by Transtympanic Electrocochleography in Auditory Neuropathy". Clin. Neurophysiol. 119(5): 1028-1041.

Schindelin, J., Arganda-Carreras, I., Frise, E., Kaynig, V., Longair, M., Pietzsch, T., Preibisch, S., Rueden, C., Saalfeld, S., Schmid, B., Tinevez, J.-Y., White, D. J., Hartenstein, V., Eliceiri, K., Tomancak, P., and Cardona, A. (2012). "Fiji: An OpenSource Platform for Biological-Image Analysis". Nat. Methods. 9(7): 676-682.

Schnupp, J. W. H., Garcia-Lazaro, J. A., and Lesica, N. A. (2015). "Periodotopy in the Gerbil Inferior Colliculus: Local Clustering Rather than a Gradient Map". Front. Neural Circuits. 9(37): 1-21. 
Schofield, B. R. (2005). "Superior Olivary Complex and Lateral Lemniscal Connections of the Auditory Midbrain". In: Winer, J. A. and Schreiner, C. E. (Eds.) "The Inferior Colliculus": 132-154. Springer-Verlag New York, New York.

Schreiner, C. E. and Langner, G. (1997). "Laminar Fine Structure of Frequency Organization in Auditory Midbrain". Nature. 388(6640): 383-386.

Schreiner, C. E. and Winer, J. A. (2007). "Auditory Cortex Mapmaking: Principles, Projections, and Plasticity". Neuron. 56(2): 356-365.

Schwander, M., Sczaniecka, A., Grillet, N., Bailey, J. S., Avenarius, M., Najmabadi, H., Steffy, B. M., Federe, G. C., Lagler, E. A., Banan, R., Hice, R., Grabowski-Boase, L., Keithley, E. M., Ryan, A. F., Housley, G. D., Wiltshire, T., Smith, R. J. H., Tarantino, L. M., and Müller, U. (2007). "A Forward Genetics Screen in Mice Identifies Recessive Deafness Traits and Reveals That Pejvakin Is Essential for Outer Hair Cell Function". J. Neurosci. 27(9): 2163-2175.

Schweitzer, V. G. and Shepard, N. (1989). "Sudden Hearing Loss: An Uncommon Manifestation of Multiple Sclerosis". Otolaryngol. Head Neck Surg. 100(4): 327-332.

Shannon, R. V., Zeng, F. G., Kamath, V., Wygonski, J., and Ekelid, M. (1995). "Speech Recognition with Primarily Temporal Cues". Science. 270(5234): 303-304.

Shearer, A. E. and Smith, R. J. (2008). "OTOF-Related Deafness". In: Adam, M. P., Ardinger, H. H., Pagon, R. A., Wallace, S. E., Bean, L. J., Stephens, K., and Amemiya, A. GeneReviews(R. [Updated 2015 Jul 30]. University of Washington, Seattle. Available from: URL: http://www.ncbi.nlm.nih.gov/books/NBK1251/.

Shehata-Dieler, W., Völter, C., Hildmann, A., Hildmann, H., and Helms, J. (2007). "[Clinical and audiological findings in children with auditory neuropathy]". Laryngorhinootologie. 86(1): 15-21.

Shrestha, B. R., Chia, C., Wu, L., Kujawa, S. G., Liberman, M. C., and Goodrich, L. V. (2018). "Sensory Neuron Diversity in the Inner Ear Is Shaped by Activity". Cell. 174(5): $1229-1246$.

Siegel, J. (1992). "Spontaneous Synaptic Potentials from Afferent Terminals in the Guinea Pig Cochlea". Hear. Res. 59(1): 85-92.

Sloan, T. B. (1998). "Anesthetic Effects on Electrophysiologic Recordings". J. Clin. Neurophysiol. 15(3): 217-226.

Smith, D. I. and Mills, J. H. (1989). "Anesthesia Effects: Auditory Brain-Stem Response". Electroencephalogr. Clin. Neurophysiol. 72(5): 422-428.

Smith, P. H., Joris, P. X., and Yin, T. C. (1993). "Projections of Physiologically Characterized Spherical Bushy Cell Axons from the Cochlear Nucleus of the Cat: Evidence for Delay Lines to the Medial Superior Olive". J. Comp. Neurol. 331(2): 245-260.

Smith, P. H. and Rhode, W. S. (1989). "Structural and Functional Properties Distinguish Two Types of Multipolar Cells in the Ventral Cochlear Nucleus". J. Comp. Neurol. 282(4): 595-616.

Smith, P. H., Joris, P. X., Carney, L. H., and Yin, T. C. T. (1991). "Projections of Physiologically Characterized Globular Bushy Cell Axons from the Cochlear Nucleus of the Cat". J. Comp. Neurol. 304(3): 387-407.

Smith, R. J., Bale Jr, J. F., and White, K. R. (2005). "Sensorineural Hearing Loss in Children". Lancet. 365(9462): 879-890. 
Spirou, G. A., Brownell, W. E., and Zidanic, M. (1990). "Recordings from Cat Trapezoid Body and HRP Labeling of Globular Bushy Cell Axons". J. Neurophysiol. 63(5): 1169-1190.

Stalmann, U. (2015). "[Altersabhängige Degeneration Und Lärmempfindlichkeit Des CortiOrgans Bei Tauben Otof Knockout Mäusen]" (PhD Thesis).

Stange-Marten, A., Nabel, A. L., Sinclair, J. L., Fischl, M., Alexandrova, O., Wohlfrom, H., Kopp-Scheinpflug, C., Pecka, M., and Grothe, B. (2017). "Input Timing for Spatial Processing Is Precisely Tuned via Constant Synaptic Delays and Myelination Patterns in the Auditory Brainstem". Proc. Natl. Acad. Sci. U.S.A. 114(24): E4851E4858.

Starr, A., Picton, T. W., Sininger, Y., Hood, L. J., and Berlin, C. I. (1996). "Auditory Neuropathy". Brain. 119(3): 741-754.

Stiebler, I. and Ehret, G. (1985). "Inferior Colliculus of the House Mouse. I. A Quantitative Study of Tonotopic Organization, Frequency Representation, and ToneThreshold Distribution". J. Comp. Neurol. 238(1): 65-76.

Strenzke, N., Chakrabarti, R., Al-Moyed, H., Müller, A., Hoch, G., Pangrsic, T., Yamanbaeva, G., Lenz, C., Pan, K.-T., Auge, E., Geiss-Friedlander, R., Urlaub, H., Brose, N., Wichmann, C., and Reisinger, E. (2016). "Hair Cell Synaptic Dysfunction, Auditory Fatigue and Thermal Sensitivity in Otoferlin Ile515Thr Mutants". EMBO J. 35(23): 2519-2535.

Strenzke, N., Chanda, S., Kopp-Scheinpflug, C., Khimich, D., Reim, K., Bulankina, A. V., Neef, A., Wolf, F., Brose, N., Xu-Friedman, M. A., and Moser, T. (2009). "Complexin-I Is Required for High-Fidelity Transmission at the Endbulb of Held Auditory Synapse". J. Neurosci. 29(25): 7991-8004.

Swaminathan, J. and Heinz, M. G. (2012). "Psychophysiological Analyses Demonstrate the Importance of Neural Envelope Coding for Speech Perception in Noise". J. Neurosci. 32(5): 1747-1756.

Taberner, A. M. (2005). "Using Knockout Mice to Study the Molecular Mechanisms That Shape Auditory Nerve Responses" (PhD thesis).

Taberner, A. M. and Liberman, M. C. (2005). "Response Properties of Single Auditory Nerve Fibers in the Mouse". J. Neurophysiol. 93(1): 557-569.

Tan, M. L., Theeuwes, H. P., Feenstra, L., and Borst, J. G. G. (2007). "Membrane Properties and Firing Patterns of Inferior Colliculus Neurons: An in Vivo PatchClamp Study in Rodents". J. Neurophysiol. 98(1): 443-453.

Tekin, M., Akcayoz, D., and Incesulu, A. (2005). "A Novel Missense Mutation in a C2 Domain of OTOF Results in Autosomal Recessive Auditory Neuropathy". Am. J. Med. Genet. A. $138 \mathrm{~A}(1)$ : 6-10.

Tian, Y., Li, M., Fritzsch, B., and Zuo, J. (2004). "Creation of a Transgenic Mouse for Hair-Cell Gene Targeting by Using a Modified Bacterial Artificial Chromosome Containing Prestin". Dev. Dyn. 231(1): 199-203.

Tranebjærg, L., Strenzke, N., Lindholm, S., Rendtorff, N. D., Poulsen, H., Khandelia, H., Kopec, W., Lyngbye, T. J. B., Hamel, C., Delettre, C., Bocquet, B., Bille, M., Owen, H. H., Bek, T., Jensen, H., Østergaard, K., Möller, C., Luxon, L., Carr, L., Wilson, L., Rajput, K., Sirimanna, T., Harrop-Griffiths, K., Rahman, S., Vona, B., Doll, J., Haaf, T., Bartsch, O., Rosewich, H., Moser, T., and Bitner-Glindzicz, M. (2018). "The CAPOS Mutation in ATP1A3 Alters Na/K-ATPase Function and 
Results in Auditory Neuropathy Which Has Implications for Management". Hum. Genet. 137(2): 111-127.

Uthaiah, R. C. and Hudspeth, A. J. (2010). "Molecular Anatomy of the Hair Cell's Ribbon Synapse". J. Neurosci. 30(37): 12387-12399.

Van Hemmen, J. L. (2013). "Vector Strength after Goldberg, Brown, and von Mises: Biological and Mathematical Perspectives". Biol. Cybern. 107(4): 385-396.

Varga, R., Avenarius, M. R., Kelley, P. M., Keats, B. J., Berlin, C. I., Hood, L. J., Morlet, T. G., Brashears, S. M., Starr, A., Cohn, E. S., Smith, R. J. H., and Kimberling, W. J. (2006). "OTOF Mutations Revealed by Genetic Analysis of Hearing Loss Families Including a Potential Temperature Sensitive Auditory Neuropathy Allele". J. Med. Genet. 43(7): 576-581.

Vogl, C., Cooper, B. H., Neef, J., Wojcik, S. M., Reim, K., Reisinger, E., Brose, N., Rhee, J.-S., Moser, T., and Wichmann, C. (2015). "Unconventional Molecular Regulation of Synaptic Vesicle Replenishment in Cochlear Inner Hair Cells". J. Cell Sci. 128(4): 638-644.

Von Gersdorff, H. and Matthews, G. (1994). "Dynamics of Synaptic Vesicle Fusion and Membrane Retrieval in Synaptic Terminals". Nature. 367(6465): 735-739.

Von Gersdorff, H. and Borst, J. G. G. (2002). "Short-Term Plasticity at the Calyx of Held". Nat. Rev. Neurosci. 3(1): 53-64.

Wagner, T. (1994). "Intrinsic Properties of Identified Neurones in the Central Nucleus of Mouse Inferior Colliculus." Neuroreport. 6(1): 89-93.

Walton, J. P., Barsz, K., and Wilson, W. W. (2008). "Sensorineural Hearing Loss and Neural Correlates of Temporal Acuity in the Inferior Colliculus of the C57BL/6 Mouse". J. Assoc. Res. Otolaryngol. 9(1): 90-101.

Wang, C.-C., Ortiz-González, X. R., Yum, S. W., Gill, S. M., White, A., Kelter, E., Seaver, L. H., Lee, S., Wiley, G., Gaffney, P. M., Wierenga, K. J., and Rasband, M. N. (2018). " $\beta$ IV Spectrinopathies Cause Profound Intellectual Disability, Congenital Hypotonia, and Motor Axonal Neuropathy". Am. J. Hum. Genet. 102(6): 11581168.

Wang, D.-Y., Wang, Y.-C., Weil, D., Zhao, Y.-L., Rao, S.-Q., Zong, L., Ji, Y.-B., Liu, Q., Li, J.-Q., Yang, H.-M., Shen, Y., Benedict-Alderfer, C., Zheng, Q.-Y., Petit, C., and Wang, Q.-J. (2010). "Screening Mutations of OTOF Gene in Chinese Patients with Auditory Neuropathy, Including a Familial Case of Temperature-Sensitive Auditory Neuropathy". BMC Med. Genet. 11(79): 1-7.

Waxman, S. G. (2006). "Axonal Conduction and Injury in Multiple Sclerosis: The Role of Sodium Channels". Nat. Rev. Neurosci. 7(12): 932-941.

Willott, J. F. and Urban, G. P. (1978). "Response Properties of Neurons in Nuclei of the Mouse Inferior Colliculus". J. Comp. Physiol. A. 127(2): 175-184.

Wilson, W. W. and Walton, J. P. (2002). "Background Noise Improves Gap Detection in Tonically Inhibited Inferior Colliculus Neurons". J. Neurophysiol. 87(1): 240-249.

Winer, J. A., Marie, R. L. S., Larue, D. T., and Oliver, D. L. (1996). "GABAergic Feedforward Projections from the Inferior Colliculus to the Medial Geniculate Body". Proc. Natl. Acad. Sci. U.S.A. 93(15): 8005-8010.

Winer, J., Miller, L., Lee, C., and Schreiner, C. (2005). "Auditory Thalamocortical Transformation: Structure and Function". Trends Neurosci. 28(5): 255-263. 
Winer, J. A. (2005). "Three Systems of Descending Projections to the Inferior Colliculus". In: Winer, J. A. and Schreiner, C. E. (Eds.) "The Inferior Colliculus": 231-247. Springer-Verlag New York, New York.

Winer, J. A., Larue, D. T., Diehl, J. J., and Hefti, B. J. (1998). "Auditory Cortical Projections to the Cat Inferior Colliculus". J. Comp. Neurol. 400(2): 147-174.

Winter, I. M., Robertson, D., and Yates, G. K. (1990). "Diversity of Characteristic Frequency Rate-Intensity Functions in Guinea Pig Auditory Nerve Fibres". Hear. Res. 45(3): 191-202.

Wu, C.-C., Liu, T.-C., Wang, S.-H., Hsu, C.-J., and Wu, C.-M. (2011). "Genetic Characteristics in Children with Cochlear Implants and the Corresponding Auditory Performance". Laryngoscope. 121(6): 1287-1293.

Wynne, D. P., Zeng, F.-G., Bhatt, S., Michalewski, H. J., Dimitrijevic, A., and Starr, A. (2013). "Loudness Adaptation Accompanying Ribbon Synapse and Auditory Nerve Disorders". Brain. 136: 1626-1638.

Yamanbaeva, G. (2017). "Sound Encoding in Mutant Mice with Disrupted Action Potential Generation" (Phd Thesis).

Yamauchi, K.-e. and Yamadori, T. (1982). "Retinal Projection to the Inferior Colliculus in the Rat". Cells Tissues Organs. 114(4): 355-360.

Yang, Y. (2004). " $\beta$ IV Spectrins Are Essential for Membrane Stability and the Molecular Organization of Nodes of Ranvier". J. Neurosci. 24(33): 7230-7240.

Yasunaga, S., Grati, M., Cohen-Salmon, M., El-Amraoui, A., Mustapha, M., Salem, N., El-Zir, E., Loiselet, J., and Petit, C. (1999). "A Mutation in OTOF, Encoding Otoferlin, a FER-1-like Protein, Causes DFNB9, a Nonsyndromic Form of Deafness". Nat. Genet. 21(4): 363-369.

Yildirim-Baylan, M., Bademci, G., Duman, D., Ozturkmen-Akay, H., Tokgoz-Yilmaz, S., and Tekin, M. (2014). "Evidence for Genotype-Phenotype Correlation for OTOF Mutations". Int. J. Pediatr. Otorhinolaryngol. 78(6): 950-953.

Yin, T. C. and Chan, J. C. (1990). "Interaural Time Sensitivity in Medial Superior Olive of Cat". J. Neurophysiol. 64(2): 465-488.

Yoon, C. H. and Les, E. P. (1957). "Quivering, a New First Chromosome Mutation in Mice". J. Hered. 48(4): 176-180.

Zadro, C., Ciorba, A., Fabris, A., Morgutti, M., Trevisi, P., Gasparini, P., and Martini, A. (2010). "Five New OTOF Gene Mutations and Auditory Neuropathy". Int. J. Pediatr. Otorhinolaryngol. 74(5): 494-498.

Zeng, F.-G., Kong, Y.-Y., Michalewski, H. J., and Starr, A. (2005). "Perceptual Consequences of Disrupted Auditory Nerve Activity". J. Neurophysiol. 93(6): 30503063.

Zhang, Q., Lan, L., Shi, W., Yu, L., Xie, L.-Y., Xiong, F., Zhao, C., Li, N., Yin, Z., Zong, L., Guan, J., Wang, D., Sun, W., and Wang, Q. (2016). "Temperature Sensitive Auditory Neuropathy". Hear. Res. 335: 53-63.

Zheng, J., Shen, W., He, D. Z., Long, K. B., Madison, L. D., and Dallos, P. (2000). "Prestin Is the Motor Protein of Cochlear Outer Hair Cells". Nature. 405: 149-155.

Zurita, P., Villa, A., De Ribaupierre, Y., De Ribaupierre, F., and Rouiller, E. (1994). "Changes of Single Unit Activity in the Cat's Auditory Thalamus and Cortex Associated to Different Anesthetic Conditions". Neurosci. Res. 19(3): 303-316. 



\section{B. Statistics}

Description of the column heads:

Fig - Figure and panel referred to.

Description - Description of what is tested.

S-W: Wt - Test for normality within the wildtype group by Shapiro-Wilks test.

$\mathrm{S}-\mathrm{W}: \mathrm{Mu}$ - Test for normality within the mutant group by Shapiro-Wilks test.

Lev - Test for heterogeneity of variances between groups by Levenes test.

Test used - Consequential test used to compare wildtype and mutant groups.

$$
\begin{aligned}
& \text { K-S-test }- \text { Kolmogorov-Smirnov-test } \\
& \text { K-W-test }- \text { Kruskal-Wallis-test } \\
& \text { MWU-test - Mann-Whitney-U-test } \\
& \text { T-test } \quad \text { Student's t-test } \\
& \text { Welch-test - Welch's t-test } \\
& \text { p-val } \quad \text { P-value of the test used. }
\end{aligned}
$$

\begin{tabular}{|c|c|c|c|c|c|c|c|}
\hline Fig & & Description & S-W: Wt & $\mathrm{S}-\mathrm{W}: \mathrm{Mu}$ & Lev & Test used & p-val \\
\hline \multirow{5}{*}{4.4} & A & Spontaneous rate & - & - & - & K-S-test & 0,670 \\
\hline & B & $\mathrm{CF}$ & $<0,001$ & $<0,001$ & 0,304 & MWU-test & 0,216 \\
\hline & B & Unit depth & 0,009 & 0,712 & 0,801 & MWU-test & 0,274 \\
\hline & $\mathrm{C}$ & Threshold & 0,122 & 0,022 & 0,060 & MWU-test & 0,503 \\
\hline & $\mathrm{D}$ & dBOpt & 0,002 & 0,009 & 0,328 & MWU-test & 0,022 \\
\hline \multirow[t]{6}{*}{4.5} & B & Mean rate & $<0,001$ & $<0,001$ & 0,957 & MWU-test & 0,589 \\
\hline & & Max rate & $<0,001$ & $<0,001$ & 0,769 & MWU-test & 0,626 \\
\hline & $\mathrm{C}$ & Dynamic range & 0,038 & 0,005 & 0,012 & K-W-test & 0,022 \\
\hline & $\mathrm{E}$ & Mean rate & $<0,001$ & 0,015 & 0,148 & MWU-test & 0,849 \\
\hline & & Max rate & $<0,001$ & $<0,001$ & 0,228 & MWU-test & 0,647 \\
\hline & $\mathrm{F}$ & Dynamic range & 0,027 & $<0,001$ & 0,365 & MWU-test & 0,004 \\
\hline & & Continued & xt page. . . & & & & \\
\hline
\end{tabular}

Table 23.: Statistics of Otof ${ }^{1515 T / I 515 T}$ mice 


\begin{tabular}{|c|c|c|c|c|c|c|c|}
\hline \multicolumn{8}{|c|}{ Statistics of Otof ${ }^{I 515 T / I 515 T}$ mice } \\
\hline \multicolumn{2}{|c|}{ Fig } & Description & S-W: Wt & $\mathrm{S}-\mathrm{W}: \mathrm{Mu}$ & Lev & Test used & p-val \\
\hline & A & All & $<0,001$ & $<0,001$ & 0,794 & MWU-test & 0,384 \\
\hline & B & Onset & $<0,001$ & $<0,001$ & 0,976 & MWU-test & 0,852 \\
\hline & $\mathrm{C}$ & Sustained & $<0,001$ & $<0,001$ & 0,720 & MWU-test & 0,456 \\
\hline \multirow[t]{4}{*}{4.7} & B & Peak-rate & 0,643 & 0,146 & 0,586 & T-test & 0,008 \\
\hline & $\mathrm{C}$ & Steady-state rate & $<0,001$ & $<0,001$ & 0,400 & MWU-test & 0,328 \\
\hline & $\mathrm{E}$ & First-spike latency & $<0,001$ & $<0,001$ & 0,512 & MWU-test & 0,136 \\
\hline & $\mathrm{F}$ & Jitter & $<0,001$ & $<0,001$ & 0,836 & MWU-test & 0,013 \\
\hline \multirow{10}{*}{\multicolumn{2}{|c|}{$4.10 \mathrm{~B}$}} & \multicolumn{6}{|c|}{ Modulation frequencies: Spike rate } \\
\hline & & $2 \mathrm{~Hz}$ & 0,001 & $<0,001$ & 0,633 & MWU-test & 0,589 \\
\hline & & $4 \mathrm{~Hz}$ & 0,001 & 0,007 & 0,605 & MWU-test & 0,484 \\
\hline & & $8 \mathrm{~Hz}$ & 0,003 & 0,020 & 0,608 & MWU-test & 0,063 \\
\hline & & $16 \mathrm{~Hz}$ & 0,016 & 0,024 & 0,511 & MWU-test & 0,026 \\
\hline & & $32 \mathrm{~Hz}$ & 0,009 & 0,007 & 0,636 & MWU-test & 0,007 \\
\hline & & $64 \mathrm{~Hz}$ & 0,008 & 0,004 & 0,260 & MWU-test & 0,011 \\
\hline & & $128 \mathrm{~Hz}$ & 0,004 & 0,006 & 0,250 & MWU-test & 0,063 \\
\hline & & $256 \mathrm{~Hz}$ & 0,001 & $<0,001$ & 0,273 & MWU-test & 0,043 \\
\hline & & $512 \mathrm{~Hz}$ & $<0,001$ & $<0,001$ & 0,507 & MWU-test & 0,123 \\
\hline \multirow{9}{*}{\multicolumn{2}{|c|}{$\mathrm{C}$}} & Modulation frequen & es: Decay ra & & & & \\
\hline & & $4 \mathrm{~Hz}$ & 0,200 & 0,560 & 0,321 & T-test & 0,016 \\
\hline & & $8 \mathrm{~Hz}$ & 0,804 & 0,707 & 0,865 & T-test & 0,002 \\
\hline & & $16 \mathrm{~Hz}$ & 0,070 & 0,119 & 0,332 & T-test & 0,001 \\
\hline & & $32 \mathrm{~Hz}$ & 0,922 & 0,019 & 0,685 & MWU-test & 0,003 \\
\hline & & $64 \mathrm{~Hz}$ & 0,201 & 0,008 & 0,226 & MWU-test & 0,005 \\
\hline & & $128 \mathrm{~Hz}$ & 0,534 & 0,038 & 0,582 & MWU-test & 0,193 \\
\hline & & $256 \mathrm{~Hz}$ & 0,026 & 0,239 & 0,444 & MWU-test & 0,484 \\
\hline & & $512 \mathrm{~Hz}$ & 0,363 & 0,246 & 0,866 & T-test & 0,580 \\
\hline \multirow{10}{*}{\multicolumn{2}{|c|}{$\mathrm{D}$}} & Modulation frequen & es: Failure $\mathrm{r}$ & & & & \\
\hline & & $2 \mathrm{~Hz}$ & $<0,001$ & $<0,001$ & 0,858 & MWU-test & 0,391 \\
\hline & & $4 \mathrm{~Hz}$ & $<0,001$ & $<0,001$ & 0,648 & MWU-test & 0,809 \\
\hline & & $8 \mathrm{~Hz}$ & $<0,001$ & $<0,001$ & 0,244 & MWU-test & 0,102 \\
\hline & & $16 \mathrm{~Hz}$ & 0,002 & 0,047 & 0,013 & $\mathrm{~K}$-W-test & 0,044 \\
\hline & & $32 \mathrm{~Hz}$ & 0,008 & 0,047 & 0,113 & MWU-test & 0,006 \\
\hline & & $64 \mathrm{~Hz}$ & 0,032 & 0,013 & 0,566 & MWU-test & 0,005 \\
\hline & & $128 \mathrm{~Hz}$ & 0,106 & 0,005 & 0,275 & MWU-test & 0,072 \\
\hline & & $256 \mathrm{~Hz}$ & 0,001 & $<0,001$ & 0,284 & MWU-test & 0,052 \\
\hline & & $512 \mathrm{~Hz}$ & $<0,001$ & $<0,001$ & 0,495 & MWU-test & 0,133 \\
\hline \multirow{3}{*}{\multicolumn{2}{|c|}{$\mathrm{E}$}} & Modulation frequen & es: Vector st & rength & & & \\
\hline & & $2 \mathrm{~Hz}$ & 0,381 & 0,005 & 0,978 & MWU-test & 0,036 \\
\hline & & $4 \mathrm{~Hz}$ & 0,045 & 0,081 & 0,914 & MWU-test & 0,327 \\
\hline
\end{tabular}




\begin{tabular}{|c|c|c|c|c|c|c|}
\hline \multicolumn{7}{|c|}{ Statistics of Otof ${ }^{1515 T / I 515 T}$ mice } \\
\hline Fig & Description & S-W: Wt & $\mathrm{S}-\mathrm{W}: \mathrm{Mu}$ & Lev & Test used & p-val \\
\hline & $8 \mathrm{~Hz}$ & 0,021 & 0,029 & 0,791 & MWU-test & 0,535 \\
\hline & $16 \mathrm{~Hz}$ & 0,649 & 0,088 & 0,399 & T-test & 0,068 \\
\hline & $32 \mathrm{~Hz}$ & 0,791 & 0,037 & 0,668 & MWU-test & 0,952 \\
\hline & $64 \mathrm{~Hz}$ & 0,161 & 0,138 & 0,074 & T-test & 0,943 \\
\hline & $128 \mathrm{~Hz}$ & 0,407 & 0,915 & 0,247 & T-test & 0,912 \\
\hline & $256 \mathrm{~Hz}$ & 0,005 & 0,594 & 0,277 & MWU-test & 0,207 \\
\hline & $512 \mathrm{~Hz}$ & 0,694 & 0,036 & 0,709 & MWU-test & 0,509 \\
\hline \multirow[t]{8}{*}{$4.11 \mathrm{~B}$} & Modulation i & es: Spike rate & & & & \\
\hline & $30 \mathrm{~dB}$ SPL & 0,076 & $<0,001$ & 0,679 & MWU-test & 1,000 \\
\hline & $40 \mathrm{~dB}$ SPL & 0,022 & $<0,001$ & 0,783 & MWU-test & 0,960 \\
\hline & $50 \mathrm{~dB}$ SPL & 0,030 & $<0,001$ & 0,443 & MWU-test & 0,879 \\
\hline & $60 \mathrm{~dB}$ SPL & 0,056 & 0,043 & 0,354 & MWU-test & 0,880 \\
\hline & $70 \mathrm{~dB}$ SPL & 0,259 & 0,016 & 0,335 & MWU-test & 0,651 \\
\hline & $80 \mathrm{~dB}$ SPL & 0,681 & 0,011 & 0,171 & MWU-test & 0,451 \\
\hline & $90 \mathrm{~dB}$ SPL & 0,928 & $<0,001$ & 0,423 & MWU-test & 0,074 \\
\hline \multirow[t]{8}{*}{$\mathrm{C}$} & Modulation i & es: Decay rat & & & & \\
\hline & 30 dB SPL & 0,643 & 0,026 & 0,924 & MWU-test & 0,454 \\
\hline & $40 \mathrm{~dB}$ SPL & 0,025 & 0,071 & 0,362 & MWU-test & 0,723 \\
\hline & $50 \mathrm{~dB}$ SPL & 0,254 & 0,092 & 0,870 & T-test & 0,685 \\
\hline & $60 \mathrm{~dB}$ SPL & 0,603 & 0,061 & 0,077 & T-test & 0,231 \\
\hline & $70 \mathrm{~dB}$ SPL & 0,910 & 0,040 & 0,238 & MWU-test & 0,580 \\
\hline & $80 \mathrm{~dB}$ SPL & 0,513 & 0,068 & 0,478 & T-test & 0,879 \\
\hline & $90 \mathrm{~dB}$ SPL & 0,320 & 0,302 & 0,539 & T-test & 0,840 \\
\hline \multirow[t]{8}{*}{$\mathrm{D}$} & Modulation i & es: Failure ra & & & & \\
\hline & 30 dB SPL & 0,097 & $<0,001$ & 0,382 & MWU-test & 0,864 \\
\hline & $40 \mathrm{~dB}$ SPL & 0,066 & 0,001 & 0,714 & MWU-test & 0,649 \\
\hline & $50 \mathrm{~dB}$ SPL & 0,044 & 0,122 & 0,432 & MWU-test & 0,648 \\
\hline & $60 \mathrm{~dB}$ SPL & 0,146 & 0,159 & 0,251 & T-test & 0,599 \\
\hline & $70 \mathrm{~dB}$ SPL & 0,017 & 0,130 & 0,806 & MWU-test & 0,513 \\
\hline & $80 \mathrm{~dB}$ SPL & 0,004 & 0,050 & 0,848 & MWU-test & 0,387 \\
\hline & 90 dB SPL & $<0,001$ & 0,485 & 0,597 & MWU-test & 0,092 \\
\hline \multirow[t]{8}{*}{$\mathrm{E}$} & Modulation i & es: Vector str & ength & & & \\
\hline & 30 dB SPL & - & - & - & - & - \\
\hline & $40 \mathrm{~dB}$ SPL & 0,402 & 0,027 & 0,335 & MWU-test & 0,799 \\
\hline & $50 \mathrm{~dB}$ SPL & 0,150 & 0,648 & 0,503 & T-test & 0,968 \\
\hline & $60 \mathrm{~dB}$ SPL & 0,873 & 0,670 & 0,709 & T-test & 0,782 \\
\hline & $70 \mathrm{~dB}$ SPL & 0,723 & 0,289 & 0,809 & T-test & 0,572 \\
\hline & $80 \mathrm{~dB}$ SPL & 0,469 & 0,763 & 0,825 & T-test & 0,701 \\
\hline & 90 dB SPL & 0,285 & 0,857 & 0,757 & T-test & 0,976 \\
\hline \multirow[t]{2}{*}{$4.12 \mathrm{~B}$} & Modulation & pike rate & & & & \\
\hline & $0 \%$ & 0,089 & 0,155 & 0,364 & T-test & 0,416 \\
\hline
\end{tabular}




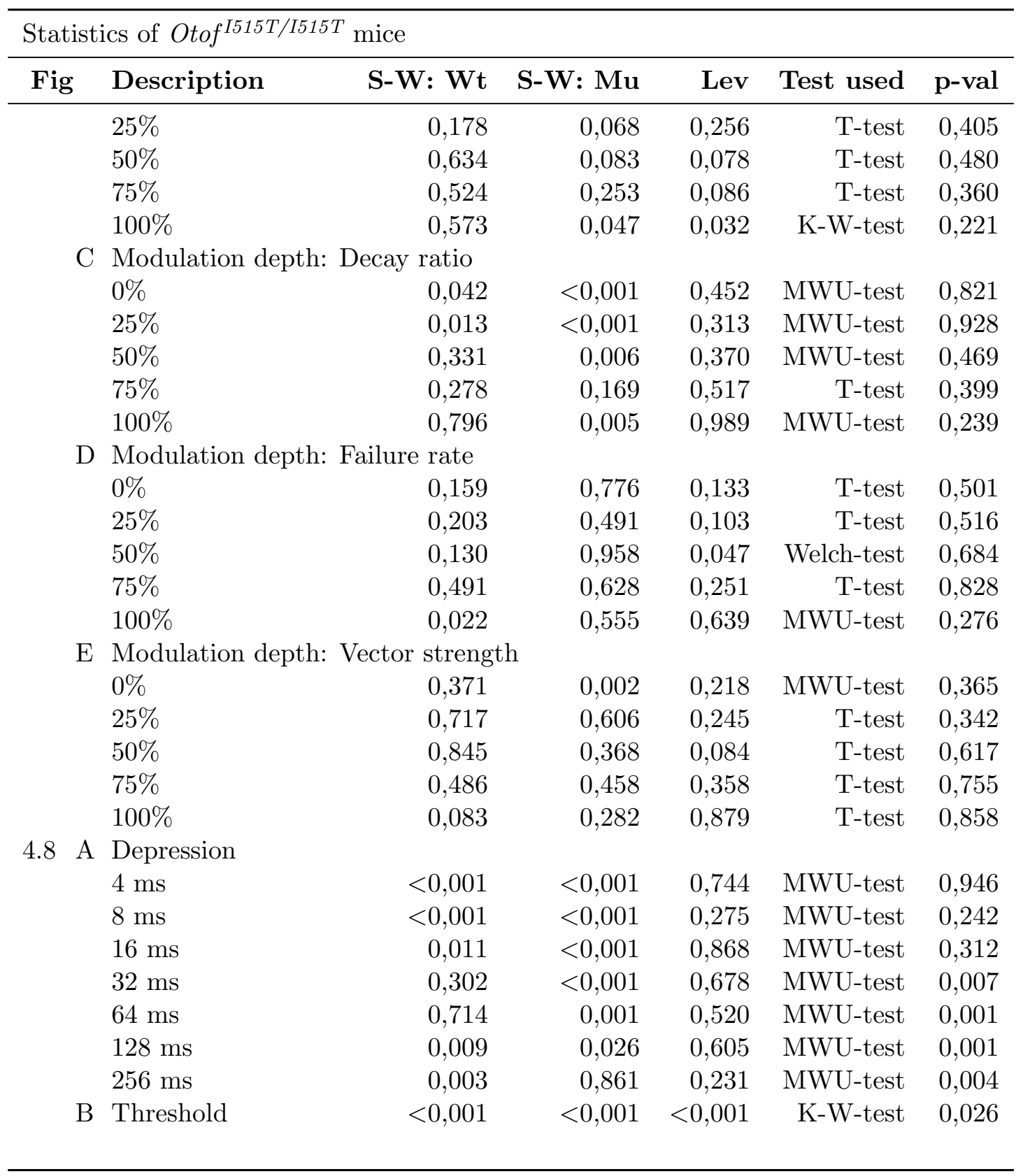

Table 24.: Statistics of $S p n b^{q v-3 J / q v-3 J}$ mice

\begin{tabular}{clrrrrr}
\hline Fig & Description & S-W: Wt & S-W: $\mathbf{M u}$ & Lev & Test used & p-val \\
\hline 4.15 A & Spontaneous rate & - & - & - & K-S-test & $<0,001$ \\
B & CF & 0,064 & 0,010 & 0,832 & MWU-test & 0,814 \\
B & Unit depth & 0,001 & 0,002 & 0,002 & K-W-test & 0,078 \\
C & Threshold & 0,226 & 0,208 & 0,061 & T-test & 0,480 \\
\hline
\end{tabular}

Continued on next page... 


\begin{tabular}{|c|c|c|c|c|c|c|}
\hline \multicolumn{7}{|c|}{ Statistics of $S p n b^{q v-3 J / q v-3 J}$ mice } \\
\hline Fig & Description & S-W: Wt & $\mathrm{S}-\mathrm{W}: \mathrm{Mu}$ & Lev & Test used & p-val \\
\hline $\mathrm{D}$ & dBOpt & 0,001 & 0,001 & 0,200 & MWU-test & 0,736 \\
\hline \multirow[t]{2}{*}{$4.16 \mathrm{~B}$} & Mean rate & $<0,001$ & $<0,001$ & 0,656 & MWU-test & 0,362 \\
\hline & Max rate & $<0,001$ & 0,003 & 0,891 & MWU-test & 0,279 \\
\hline $\mathrm{C}$ & Dynamic range & 0,041 & 0,017 & 0,723 & MWU-test & 0,571 \\
\hline \multirow[t]{2}{*}{$\mathrm{E}$} & Mean rate & 0,003 & $<0,001$ & 0,064 & MWU-test & 0,253 \\
\hline & Max rate & 0,005 & $<0,001$ & 0,044 & K-W-test & 0,227 \\
\hline $\mathrm{F}$ & Dynamic range & 0,286 & 0,617 & 0,558 & t-test & 0,450 \\
\hline $4.17 \mathrm{~A}$ & All & $<0,001$ & $<0,001$ & 0,255 & MWU-test & 0,045 \\
\hline B & Onset & $<0,001$ & $<0,001$ & 0,863 & MWU-test & 0,905 \\
\hline $\mathrm{C}$ & Sustained & $<0,001$ & $<0,001$ & 0,433 & MWU-test & 0,110 \\
\hline $4.18 \mathrm{~B}$ & Peak-rate & 0,004 & 0,008 & 0,027 & $\mathrm{~K}$-W-test & 0,003 \\
\hline $\mathrm{C}$ & Steady-state rate & $<0,001$ & $<0,001$ & 0,076 & MWU-test & 0,121 \\
\hline $\mathrm{E}$ & First-spike latency & $<0,001$ & $<0,001$ & 0,146 & MWU-test & $<0,001$ \\
\hline $\mathrm{F}$ & Jitter & $<0,001$ & $<0,001$ & 0,311 & MWU-test & 0,212 \\
\hline $4.19 \mathrm{~B}$ & Peak-rate & 0,006 & 0,001 & 0,068 & MWU-test & 0,001 \\
\hline $\mathrm{C}$ & Steady-state rate & $<0,001$ & $<0,001$ & 0,640 & MWU-test & 0,074 \\
\hline $\mathrm{E}$ & First-spike latency & 0,001 & 0,010 & 0,001 & K-W-test & $<0,001$ \\
\hline $\mathrm{F}$ & Jitter & $<0,001$ & $<0,001$ & 0,006 & $\mathrm{~K}$-W-test & 0,003 \\
\hline \multirow[t]{10}{*}{$4.22 \mathrm{~B}$} & \multicolumn{6}{|c|}{ Modulation frequencies: Spike rate } \\
\hline & $2 \mathrm{~Hz}$ & 0,006 & $<0,001$ & 0,786 & MWU-test & 0,065 \\
\hline & $4 \mathrm{~Hz}$ & 0,018 & $<0,001$ & 0,948 & MWU-test & 0,035 \\
\hline & $8 \mathrm{~Hz}$ & 0,160 & $<0,001$ & 0,809 & MWU-test & 0,037 \\
\hline & $16 \mathrm{~Hz}$ & 0,078 & $<0,001$ & 0,655 & MWU-test & 0,101 \\
\hline & $32 \mathrm{~Hz}$ & 0,007 & $<0,001$ & 0,606 & MWU-test & 0,268 \\
\hline & $64 \mathrm{~Hz}$ & 0,077 & $<0,001$ & 0,502 & MWU-test & 0,425 \\
\hline & $128 \mathrm{~Hz}$ & 0,051 & $<0,001$ & 0,898 & MWU-test & 0,391 \\
\hline & $256 \mathrm{~Hz}$ & 0,523 & $<0,001$ & 0,584 & MWU-test & 0,976 \\
\hline & $512 \mathrm{~Hz}$ & 0,073 & $<0,001$ & 0,874 & MWU-test & 0,574 \\
\hline \multirow[t]{8}{*}{$\mathrm{C}$} & \multicolumn{6}{|c|}{ Modulation frequencies: Decay ratio } \\
\hline & $4 \mathrm{~Hz}$ & 0,336 & 0,017 & 0,891 & MWU-test & 0,160 \\
\hline & $8 \mathrm{~Hz}$ & 0,042 & 0,037 & 0,925 & MWU-test & 0,135 \\
\hline & $16 \mathrm{~Hz}$ & 0,060 & 0,026 & 0,387 & MWU-test & 0,647 \\
\hline & $32 \mathrm{~Hz}$ & 0,512 & 0,082 & 0,757 & T-test & 0,381 \\
\hline & $64 \mathrm{~Hz}$ & 0,709 & 0,097 & 0,932 & T-test & 0,283 \\
\hline & $128 \mathrm{~Hz}$ & 0,601 & 0,003 & 0,675 & MWU-test & 0,668 \\
\hline & $256 \mathrm{~Hz}$ & 0,262 & 0,506 & 0,616 & T-test & 0,960 \\
\hline
\end{tabular}




\begin{tabular}{|c|c|c|c|c|c|c|}
\hline \multicolumn{7}{|c|}{ Statistics of $S p n b^{q v-3 J / q v-3 J}$ mice } \\
\hline Fig & Description & S-W: Wt & S-W: Mu & Lev & Test used & p-val \\
\hline \multirow{11}{*}{$\mathrm{D}$} & $512 \mathrm{~Hz}$ & 0,989 & 0,324 & 0,852 & T-test & 0,957 \\
\hline & \multicolumn{6}{|c|}{ Modulation frequencies: Failure rate } \\
\hline & $2 \mathrm{~Hz}$ & $<0,001$ & $<0,001$ & 0,234 & MWU-test & 0,058 \\
\hline & $4 \mathrm{~Hz}$ & $<0,001$ & 0,005 & 0,126 & MWU-test & 0,025 \\
\hline & $8 \mathrm{~Hz}$ & $<0,001$ & 0,035 & 0,096 & MWU-test & 0,014 \\
\hline & $16 \mathrm{~Hz}$ & 0,031 & 0,007 & 0,780 & MWU-test & 0,135 \\
\hline & $32 \mathrm{~Hz}$ & 0,028 & $<0,001$ & 0,471 & MWU-test & 0,255 \\
\hline & $64 \mathrm{~Hz}$ & 0,143 & $<0,001$ & 0,407 & MWU-test & 0,322 \\
\hline & $128 \mathrm{~Hz}$ & 0,075 & $<0,001$ & 0,952 & MWU-test & 0,408 \\
\hline & $256 \mathrm{~Hz}$ & 0,193 & $<0,001$ & 0,580 & MWU-test & 0,859 \\
\hline & $512 \mathrm{~Hz}$ & 0,066 & $<0,001$ & 0,880 & MWU-test & 0,574 \\
\hline \multirow[t]{10}{*}{$\mathrm{E}$} & \multicolumn{6}{|c|}{ Modulation frequencies: Vector strength } \\
\hline & $2 \mathrm{~Hz}$ & 0,464 & 0,262 & 0,791 & T-test & 0,523 \\
\hline & $4 \mathrm{~Hz}$ & 0,049 & 0,102 & 0,801 & MWU-test & 0,544 \\
\hline & $8 \mathrm{~Hz}$ & 0,188 & 0,055 & 0,365 & T-test & 0,903 \\
\hline & $16 \mathrm{~Hz}$ & 0,100 & 0,274 & 0,151 & T-test & 0,682 \\
\hline & $32 \mathrm{~Hz}$ & 0,899 & 0,037 & 0,300 & MWU-test & 0,275 \\
\hline & $64 \mathrm{~Hz}$ & 0,491 & 0,011 & 0,586 & MWU-test & 0,029 \\
\hline & $128 \mathrm{~Hz}$ & 0,079 & $<0,001$ & 0,063 & MWU-test & 0,001 \\
\hline & $256 \mathrm{~Hz}$ & 0,013 & $<0,001$ & 0,467 & MWU-test & 0,372 \\
\hline & $512 \mathrm{~Hz}$ & $<0,001$ & 0,001 & 0,382 & MWU-test & 0,841 \\
\hline \multirow[t]{8}{*}{$4.23 \mathrm{~B}$} & \multicolumn{6}{|c|}{ Modulation intensities: Spike rate } \\
\hline & $30 \mathrm{~dB}$ SPL & $<0,001$ & $<0,001$ & 0,169 & MWU-test & 0,023 \\
\hline & $40 \mathrm{~dB}$ SPL & $<0,001$ & $<0,001$ & 0,130 & MWU-test & 0,009 \\
\hline & $50 \mathrm{~dB}$ SPL & $<0,001$ & $<0,001$ & 0,182 & MWU-test & 0,097 \\
\hline & $60 \mathrm{~dB}$ SPL & 0,010 & $<0,001$ & 0,362 & MWU-test & 0,293 \\
\hline & $70 \mathrm{~dB}$ SPL & 0,249 & 0,005 & 0,715 & MWU-test & 0,317 \\
\hline & $80 \mathrm{~dB}$ SPL & 0,724 & 0,002 & 0,291 & MWU-test & 0,040 \\
\hline & $90 \mathrm{~dB}$ SPL & 0,316 & 0,060 & 0,960 & T-test & 0,127 \\
\hline \multirow[t]{8}{*}{$\mathrm{C}$} & \multicolumn{6}{|c|}{ Modulation intensities: Decay ratio } \\
\hline & $30 \mathrm{~dB}$ SPL & $<0,001$ & $<0,001$ & 0,086 & MWU-test & 0,103 \\
\hline & $40 \mathrm{~dB}$ SPL & 0,134 & $<0,001$ & $<0,001$ & $\mathrm{~K}$-W-test & 0,002 \\
\hline & $50 \mathrm{~dB}$ SPL & 0,344 & $<0,001$ & 0,161 & MWU-test & 0,012 \\
\hline & $60 \mathrm{~dB}$ SPL & 0,146 & 0,463 & 0,339 & T-test & 0,026 \\
\hline & $70 \mathrm{~dB}$ SPL & 0,139 & 0,378 & 0,200 & T-test & 0,039 \\
\hline & $80 \mathrm{~dB}$ SPL & 0,026 & 0,021 & 0,146 & MWU-test & 0,001 \\
\hline & $90 \mathrm{~dB}$ SPL & 0,002 & 0,196 & 0,277 & MWU-test & 0,001 \\
\hline \multirow[t]{4}{*}{$\mathrm{D}$} & Modulation intensities: & : Failure rat & & & & \\
\hline & $30 \mathrm{~dB}$ SPL & $<0,001$ & $<0,001$ & 0,136 & MWU-test & 0,023 \\
\hline & $40 \mathrm{~dB}$ SPL & $<0,001$ & $<0,001$ & 0,068 & MWU-test & 0,009 \\
\hline & $50 \mathrm{~dB}$ SPL & 0,001 & $<0,001$ & 0,102 & MWU-test & 0,078 \\
\hline
\end{tabular}




\begin{tabular}{|c|c|c|c|c|c|c|}
\hline \multicolumn{7}{|c|}{ Statistics of $S p n b^{q v-3 J / q v-3 J}$ mice } \\
\hline Fig & Description & S-W: Wt & $\mathrm{S}-\mathrm{W}: \mathrm{Mu}$ & Lev & Test used & p-val \\
\hline & $60 \mathrm{~dB}$ SPL & 0,025 & 0,002 & 0,331 & MWU-test & 0,317 \\
\hline & 70 dB SPL & 0,082 & 0,034 & 0,537 & MWU-test & 0,151 \\
\hline & $80 \mathrm{~dB}$ SPL & 0,012 & 0,025 & 0,034 & K-W-test & 0,003 \\
\hline & $90 \mathrm{~dB}$ SPL & 0,005 & 0,506 & 0,021 & $\mathrm{~K}$-W-test & 0,005 \\
\hline \multirow[t]{8}{*}{$\mathrm{E}$} & Modulation ir & ies: Vector stı & ength & & & \\
\hline & $30 \mathrm{~dB}$ SPL & - & - & - & - & - \\
\hline & $40 \mathrm{~dB}$ SPL & - & - & - & - & - \\
\hline & $50 \mathrm{~dB}$ SPL & 0,615 & 0,037 & 0,264 & MWU-test & 0,256 \\
\hline & $60 \mathrm{~dB}$ SPL & 0,979 & 0,074 & 0,296 & T-test & 0,154 \\
\hline & $70 \mathrm{~dB}$ SPL & 0,904 & 0,952 & 0,629 & T-test & 0,935 \\
\hline & $80 \mathrm{~dB}$ SPL & 0,504 & 0,821 & 0,924 & T-test & 0,469 \\
\hline & $90 \mathrm{~dB}$ SPL & 0,515 & 0,607 & 0,519 & T-test & 0,601 \\
\hline \multirow[t]{6}{*}{$4.24 \mathrm{~B}$} & Modulation d & Spike rate & & & & \\
\hline & $0 \%$ & 0,863 & 0,001 & 0,948 & MWU-test & 0,056 \\
\hline & $25 \%$ & 0,692 & 0,001 & 0,922 & MWU-test & 0,060 \\
\hline & $50 \%$ & 0,793 & 0,003 & 0,853 & MWU-test & 0,039 \\
\hline & $75 \%$ & 0,023 & 0,009 & 0,770 & MWU-test & 0,011 \\
\hline & $100 \%$ & 0,789 & 0,006 & 0,840 & MWU-test & 0,009 \\
\hline \multirow[t]{6}{*}{$\mathrm{C}$} & Modulation d & Decay ratio & & & & \\
\hline & $0 \%$ & 0,279 & $<0,001$ & 0,378 & MWU-test & 0,148 \\
\hline & $25 \%$ & 0,184 & $<0,001$ & 0,427 & MWU-test & 0,069 \\
\hline & $50 \%$ & 0,464 & 0,002 & 0,832 & MWU-test & 0,079 \\
\hline & $75 \%$ & 0,084 & 0,003 & 0,859 & MWU-test & 0,069 \\
\hline & $100 \%$ & 0,328 & 0,013 & 0,577 & MWU-test & 0,091 \\
\hline \multirow[t]{6}{*}{$\mathrm{D}$} & Modulation d & Failure rate & & & & \\
\hline & $0 \%$ & 0,489 & 0,015 & 0,327 & MWU-test & 0,148 \\
\hline & $25 \%$ & 0,476 & 0,008 & 0,319 & MWU-test & 0,116 \\
\hline & $50 \%$ & 0,789 & 0,059 & 0,546 & T-test & 0,027 \\
\hline & $75 \%$ & 0,749 & 0,076 & 0,640 & T-test & 0,002 \\
\hline & $100 \%$ & 0,003 & 0,089 & 0,146 & MWU-test & 0,009 \\
\hline \multirow[b]{7}{*}{4} & Modulation d & Vector streng & & & & \\
\hline & $0 \%$ & 0,001 & 0,001 & 0,651 & MWU-test & 0,878 \\
\hline & $25 \%$ & 0,081 & 0,001 & 0,642 & MWU-test & 0,372 \\
\hline & $50 \%$ & 0,823 & 0,209 & 0,485 & T-test & 0,594 \\
\hline & $75 \%$ & 0,726 & 0,739 & 0,476 & T-test & 0,246 \\
\hline & $100 \%$ & 0,932 & 0,278 & 0,709 & T-test & 0,716 \\
\hline & Depression & & & & & \\
\hline \multirow{4}{*}{$4.20 \mathrm{~A}$} & $4 \mathrm{~ms}$ & $<0,001$ & $<0,001$ & 0,776 & MWU-test & 0,475 \\
\hline & $8 \mathrm{~ms}$ & 0,325 & 0,167 & 0,307 & T-test & 0,812 \\
\hline & $16 \mathrm{~ms}$ & $<0,001$ & 0,669 & 0,435 & MWU-test & 0,875 \\
\hline & $32 \mathrm{~ms}$ & 0,771 & 0,999 & 0,170 & T-test & 0,652 \\
\hline
\end{tabular}




\begin{tabular}{clrrrrr}
\hline \multicolumn{2}{l}{ Statistics of $S p n b^{q v-3 J / q v-3 J}$ mice } & & & & \\
\hline Fig & Description & S-W: Wt & S-W: $\mathbf{M u}$ & Lev & Test used & p-val \\
\hline $64 \mathrm{~ms}$ & 0,098 & 0,616 & 0,142 & T-test & 0,239 \\
& 0,262 & 0,874 & 0,466 & T-test & 0,262 \\
& $256 \mathrm{~ms}$ & 0,019 & 0,841 & 0,460 & MWU-test & 0,043 \\
B & Threshold & $<0,001$ & $<0,001$ & 0,359 & MWU-test & 0,717 \\
& & & & & \\
\hline
\end{tabular}




\section{Acknowledgment}

I would like to thank Dr. Nicola Strenzke as my supervisor for the opportunity to work in such a wonderful and inspiring laboratory together with many excellent scientists.

Furthermore, I thank Prof. Dr. Alexander Gail and Dr. Manuela Schmidt for their time, encouragement and useful advice in my thesis advisory committee.

Great thanks go to Dr. Marcus Jeschke for teaching me in vivo electrophysiological recordings from the inferior colliculus and for encouraging me to learn the python programming language and to write all the analysis scripts by myself.

I am very grateful for the technical assistance by Nina Dankenbrink-Werder for the support with genotyping, ordering and running the lab, for the infectious good mood of Nadine Dietrich, who was measuring ABRs for me and for the great help of Daniela Marschall, as an excellent animal caretaker, who was looking after my mouse lines.

Moreover, I thank Brigitte Higazi, Patricia Räke-Kügler and Susann Müller for their help and support in all administrative matters.

I would like to thank all the members of the InnerEarLab for sharing the past years with me, especially for fruitful discussions during lunchtime with Alexander Dieter, Dr. Christian Wrobel, Daniel Keppeler, Dr. Tobias Dombrowski, Dr. Antoine Huet, Dr. Astrid Klinge-Strahl and Dr. Fritz Kobe, for the interesting and diverse conversations with Hanan Al-Moyed, Andreia Cepeda and Mattia Nova and for the nice and helpful exchanges in the course of time with people from my office, especially Dennis Spormann, Dominik Riemenschneider, Dr. Kai Bodensiek, Dr. Tamas Harczos and Dr. Thomas Effertz, also for proof-reading my thesis.

Special thanks go to Dr. Christin Döring for the long friendship and mutual support since we were both in the first semester and for the help in structuring and proof-reading my dissertation.

Finally, many thanks go to my family and friends for their support, understanding and love, especially to my mother Melissa Hullmann and my sister Eva Pelgrim for their constant believe in me and to my boyfriend Alexander Knopp who always encouraged me and never failed to cheer me up when I needed it. 



\section{Declaration}

Here I declare that my doctoral thesis entitled "Central sound encoding in the inferior colliculus of mouse models for human auditory synaptopathy and neuropathy" has been written independently with no other sources and aids than quoted.

Maike Pelgrim

Göttingen, 30th of October 2018 



\section{E. Curriculum Vitae}

\section{Personal Information}

$\begin{array}{ll}\text { Name: } & \text { Maike Pelgrim } \\ \text { Date of birth } & \text { 09.05.1991 } \\ \text { Place of birth } & \text { Stuttgart } \\ \text { Nationality } & \text { German }\end{array}$

\section{Education and Training}

$\begin{array}{ll}2015 \text { - present } & \text { PhD student } \\ & \text { Department of Otolaryngology and InnerEarLab, University Medical Cen- } \\ & \text { ter, Göttingen } \\ & \text { Title: "Central sound encoding in the inferior colliculus of mouse models } \\ & \text { for human auditory synaptopathy and neuropathy" } \\ & \text { Master studies in Molecular Medicine, Georg-August-University, Göttin- } \\ & \text { gen, grade (1,6) } \\ & \text { Bachelor studies in Molecular Medicine, Georg-August-University, Göttin- } \\ & \text { gen, grade (1,9) } \\ & \text { Grammar school, Erasmus-Widmann-Gymnasium, Schwäbisch Hall, } \\ & \text { Abitur, grade (1,8) }\end{array}$

\section{Research experience}

2015 - present Member of the Collaborative Research Center (SFB 889)

"Cellular Mechanisms of Sensory Processing"

2015 Member of the DFG Priority Program (SPP1608)

"Ultrafast and temporally precise information processing: Normal and dysfunctional hearing"

2014 - $2015 \quad$ Research Assistant, Department of Otolaryngology and InnerEarLab, University Medical Center, Göttingen

2014 Institute of Neurophysiology and Cellular Biophysics, Göttingen Master thesis "Multiplex fluorescent Immunohistochemistry of the olfactory system of Xenopus laevis tadpoles using semiconductor Quantum Dots"

Summer 2013 Research rotation, Department of hematology and oncology, Functional imaging group, University Medical Center, Göttingen

Spring 2013 Research rotation, Clinical Pharmacology, University Medical Center, Göttingen

2012

Max Planck Institute for Experimental Medicine, Department of Neurogenetics, Göttingen

Bachelor thesis "Immunohistochemical Analysis of Cytoskeletal Septins in Developmental Myelination and in Models of Myelin-related Disease" 